University of Tennessee Health Science Center UTHSC Digital Commons

\title{
Functional and Genetic Analysis of Choroid Plexus Development in Zebrafish
}

Hannah Elizabeth Henson

University of Tennessee Health Science Center

Follow this and additional works at: https://dc.uthsc.edu/dissertations

Part of the Medical Cell Biology Commons

\section{Recommended Citation}

Henson, Hannah Elizabeth , "Functional and Genetic Analysis of Choroid Plexus Development in Zebrafish" (2014). Theses and Dissertations (ETD). Paper 340. http://dx.doi.org/10.21007/etd.cghs.2014.0134.

This Dissertation is brought to you for free and open access by the College of Graduate Health Sciences at UTHSC Digital Commons. It has been accepted for inclusion in Theses and Dissertations (ETD) by an authorized administrator of UTHSC Digital Commons. For more information, please contact jwelch30@uthsc.edu. 


\title{
Functional and Genetic Analysis of Choroid Plexus Development in Zebrafish
}

\begin{abstract}
The choroid plexus (CP) is an epithelial based structure localized within the brain ventricles and functions as the blood-cerebrospinal fluid barrier (BCSFB). Under normal conditions, the CP is responsible for generating the cerebrospinal fluid (CSF) and regulating its components. Abnormal CP function has been associated with neurodegenerative diseases, tumor formation in $\mathrm{CP}$ epithelia $(\mathrm{CPe})$, and hydrocephalus. Despite the significant role of the CP in these disorders, little research has been done to characterize its functional properties and genetically dissect the pathways involved in normal CP development and disease. For this study, we have utilized zebrafish, (Danio rerio), as a model system to better understand the genetic components of CP development. Their transparent nature and rapid ex utero development provide mechanisms to visualize $\mathrm{CP}$ formation that is not possible in other model systems. By utilizing an enhancer trap line, Et(cp:EGFP) sj2 that expresses the enhanced green fluorescent protein (EGFP) in CPe, we have demonstrated that the zebrafish CP possesses barrier properties such as tight junctions, transporter activity, and regulating central nervous system (CNS) homeostasis. After validating that the zebrafish CP is similar to higher vertebrates, we have initiated a genetic screen to answer questions such as: 1) what genes are needed for CP development, function, and maintenance, 2) what are the signaling pathways involved, and 3) how do these pathways interact with one another to form the BCSFB? This study is the first to employ an unbiased approach using a forward genetic screen to genetically dissect the $\mathrm{CP}$ and identify genes essential for its formation and function. As a result of this genetic analysis, we have confirmed 10 mutant lines with CP abnormalities. The mutants generated in this study will be used in future investigations to elucidate specific genes and signaling pathways essential for CP development, function, and maintenance and will provide a better understanding of how genetic mutations contribute to CP- related diseases. We have also mapped the gene for one mutant line, cp27.5, to Chromosome 21. Using a combination of classical positional cloning and whole-exome sequencing (WES), we identified the mutated gene as squamous cell carcinoma antigen recognized by T cells (sart1). sart1 plays a role in assembling the spliceosome, a multi-ribonucleoprotein complex essential for processing pre-mRNA. By characterizing cp27.5 mutants, we identified a number of proteins with altered expression levels and patterning primarily within the brain and eye. Using RNA-Seq analysis, we also identified multiple genes up-regulated or down-regulated due to the sart1 mutation. While sart1 itself was up-regulated, we also identified increased expression of genes involved in apoptosis such as tp53 and mdm2. A loss of photoreceptors and lack of retinal lamination in mutants is related to a down-regulation of vision-related genes such as phosphodiesterase $6 \mathrm{H}$, (pde6h) and opsin-1, short-wave-sensitive 1 (opn1sw1), both of which are expressed in photoreceptors. Future studies will investigate the role of sart1 in splicing and the mechanisms involved in producing the phenotypes observed in cp27.5 mutants.
\end{abstract}

\section{Document Type}

Dissertation

Degree Name

Doctor of Philosophy (PhD)

Program

Biomedical Sciences

Research Advisor

Michael R. Taylor, Ph.D. 


\section{Keywords}

choroid plexus, forward genetic screen, Sart1, zebrafish

\section{Subject Categories}

Medical Cell Biology | Medical Sciences | Medicine and Health Sciences

\section{Comments}

One year embargo expired August 2015 


\title{
Functional and Genetic Analysis of Choroid Plexus Development in Zebrafish
}

\author{
A Dissertation \\ Presented for \\ The Graduate Studies Council \\ The University of Tennessee \\ Health Science Center
}

\author{
In Partial Fulfillment \\ Of the Requirements for the Degree \\ Doctor of Philosophy \\ From The University of Tennessee
}

By

Hannah Elizabeth Henson

August 2014 
Copyright (C) 2014 by Hannah Elizabeth Henson. All rights reserved. 


\section{DEDICATION}

I would like to dedicate this work to my family, especially my parents, Robert and Christie Henson. 


\section{ACKNOWLEDGEMENTS}

I would first like to thank my mentor Dr. Michael Taylor for allowing me to work on this project. From day one, his enthusiasm for his research inspired me to join his lab and pursue my graduate career using zebrafish to study brain barrier development. He not only taught me the technical skills needed to perform my research, but also imparted life lessons that I will carry with me for years to come. He has challenged me to ask questions, think critically, and become independent. Thank you for your time and patience while training me to be a scientist who can be proud of their work, learn from their mistakes, and enjoy what they do. I will always be grateful for your investment in me as one of your first graduate students.

I also want to thank my committee members, Dr. Charles Lessman, Dr. Paul Mead, Dr. Leta Nutt, Dr. Radhakrishna Rao, and Dr. Clinton Stewart. Their commitment to my graduate education by attending meetings, providing feedback, and reading this dissertation is greatly appreciated.

My fellow lab mates, both past and present have truly shaped my graduate school experience. Thank you for your support, guidance, encouragement, and friendship. I have been blessed to work with you all. The Chemical Biology and Therapeutics Department has also provided a supportive and friendly environment which I will greatly miss. I also appreciate the Cell and Tissue Imaging Center, Hartwell Center, and Computational Biology Department for their assistance with this project. I want to especially thank Dr. Jennifer Peters and Dr. Victoria Frohlich from Cell and Tissue Imaging for their assistance. From the Computational Biology Department, I greatly appreciate the work done by Dr. Michael Wang for providing the methods for this manuscript on Whole-Exome Sequencing and for data analysis in Chapter 4 and Dr. Chunxu Qu for providing the methods described in RNA-Seq and for data analysis in Chapter 5.

None of this work would have been possible without the financial support from St. Jude Children's Research Hospital, American Lebanese and Syrian Associated Charities (ALSAC), The Hartwell Foundation, and University of Tennessee Health Science Center. Thank you for your investment in this project.

During my time here, I have made many friends that I hope will become lifelong friendships. Thank you for reminding me to have fun and take time to enjoy life. I also want to acknowledge my church family who has made my time in Memphis a memorable one. I will miss you all!

Finally, I would not be where I am today without the love and support from my family and friends back home. Your many prayers and words of encouragement have brought me through the good times and bad. I am still not sure if any of you knew exactly what I was doing here, but thank you for your support. I want to especially thank my mom, dad, brother, grandparents, great-grandparents, and extended family who 
always believed I could accomplish whatever I set my mind to. Most importantly, I want to thank God for giving me the strength, wisdom, and peace I need to face each day. 


\begin{abstract}
The choroid plexus (CP) is an epithelial-based structure localized within the brain ventricles and functions as the blood-cerebrospinal fluid barrier (BCSFB). Under normal conditions, the $\mathrm{CP}$ is responsible for generating the cerebrospinal fluid (CSF) and regulating its components. Abnormal $\mathrm{CP}$ function has been associated with neurodegenerative diseases, tumor formation in $\mathrm{CP}$ epithelia $(\mathrm{CPe})$, and hydrocephalus. Despite the significant role of the CP in these disorders, little research has been done to characterize its functional properties and genetically dissect the pathways involved in normal CP development and disease. For this study, we have utilized zebrafish, (Danio rerio), as a model system to better understand the genetic components of $\mathrm{CP}$ development. Their transparent nature and rapid ex utero development provide mechanisms to visualize CP formation that is not possible in other model systems. By utilizing an enhancer trap line, Et(cp:EGFP $)^{s j 2}$ that expresses the enhanced green fluorescent protein (EGFP) in CPe, we have demonstrated that the zebrafish $\mathrm{CP}$ possesses barrier properties such as tight junctions, transporter activity, and regulating central nervous system (CNS) homeostasis. After validating that the zebrafish $\mathrm{CP}$ is similar to higher vertebrates, we have initiated a genetic screen to answer questions such as: 1) what genes are needed for CP development, function, and maintenance, 2) what are the signaling pathways involved, and 3) how do these pathways interact with one another to form the BCSFB? This study is the first to employ an unbiased approach using a forward genetic screen to genetically dissect the $\mathrm{CP}$ and identify genes essential for its formation and function. As a result of this genetic analysis, we have confirmed 10 mutant lines with $\mathrm{CP}$ abnormalities. The mutants generated in this study will be used in future investigations to elucidate specific genes and signaling pathways essential for $\mathrm{CP}$ development, function, and maintenance and will provide a better understanding of how genetic mutations contribute to CP-related diseases. We have also mapped the gene for one mutant line, $c p 27.5$, to Chromosome 21. Using a combination of classical positional cloning and whole-exome sequencing (WES), we identified the mutated gene as squamous cell carcinoma antigen recognized by T cells (sart1). sart1 plays a role in assembling the spliceosome, a multi-ribonucleoprotein complex essential for processing pre-mRNA. By characterizing cp27.5 mutants, we identified a number of proteins with altered expression levels and patterning primarily within the brain and eye. Using RNA-Seq analysis, we also identified multiple genes up-regulated or down-regulated due to the sart1 mutation. While sart1 itself was up-regulated, we also identified increased expression of genes involved in apoptosis such as $t p 53$ and $m d m 2$. A loss of photoreceptors and lack of retinal lamination in mutants is related to a down-regulation of vision-related genes such as phosphodiesterase 6H, (pde6h) and opsin-1, short-wave-sensitive 1 (opn1sw1), both of which are expressed in photoreceptors. Future studies will investigate the role of sart1 in splicing and the mechanisms involved in producing the phenotypes observed in $c p 27.5$ mutants.
\end{abstract}




\section{TABLE OF CONTENTS}

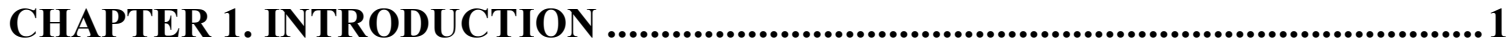

Central Nervous System Barriers ................................................................... 1

Blood-Brain Barrier ................................................................................. 1

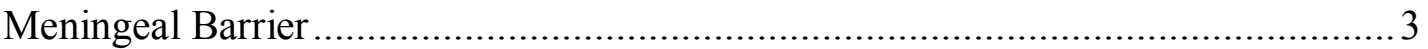

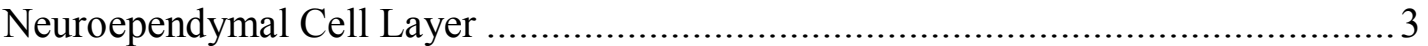

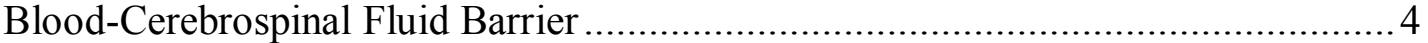

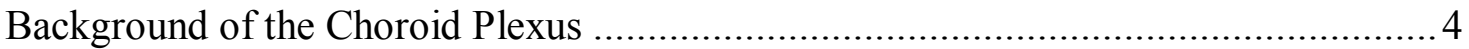

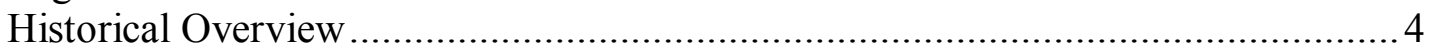

Choroid Plexus Function............................................................................. 4

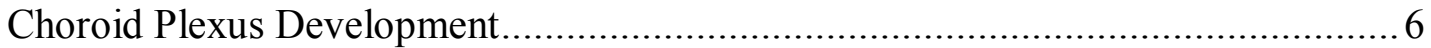

Signaling Pathways and the Choroid Plexus.................................................. 7

Choroid Plexus and Disease ...................................................................... 9

Choroid Plexus Model Systems ..................................................................... 10

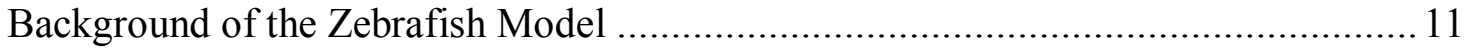

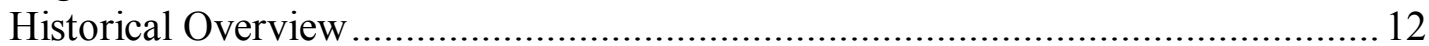

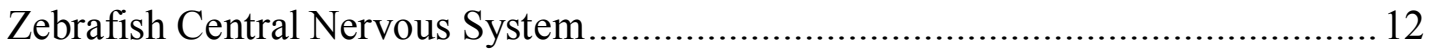

Zebrafish as a Model for Choroid Plexus Development....................................... 15

Zebrafish Genetic Screening Strategies ......................................................... 17

Using Zebrafish for Genetic Analysis ............................................................ 19

\section{CHAPTER 2. CHARACTERIZATION OF THE ZEBRAFISH CHOROID}

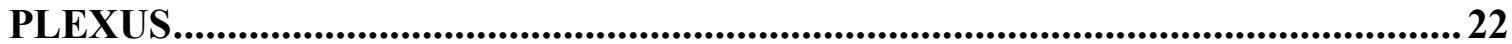

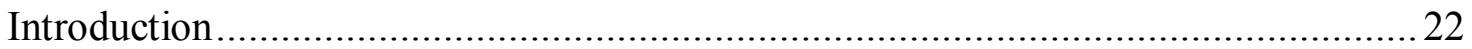

Central Nervous System Barrier Properties ........................................................... 22

Claudin Proteins Regulate Paracellular Transport ................................................ 22

Transporter Proteins Control Transcellular Permeability ..................................25

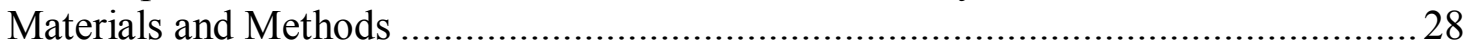

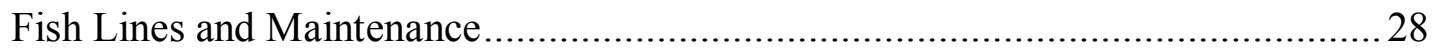

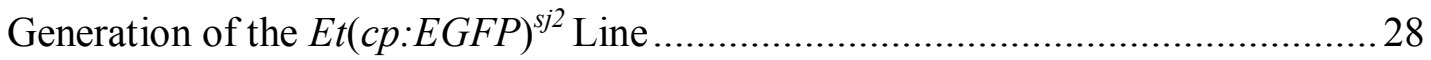

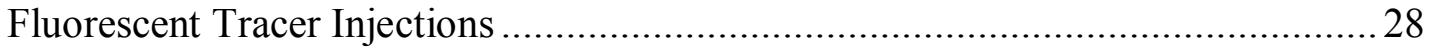

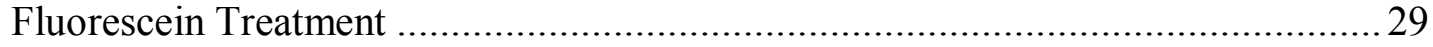

Statistical Analysis of Tracer Permeability ......................................................29

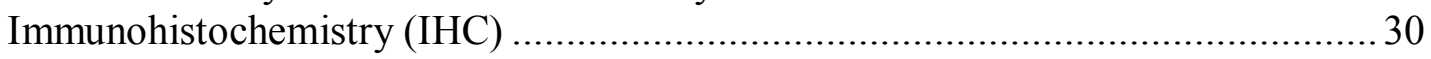

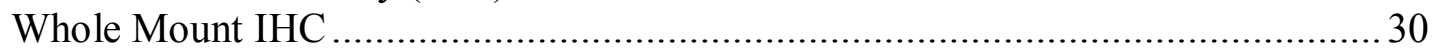

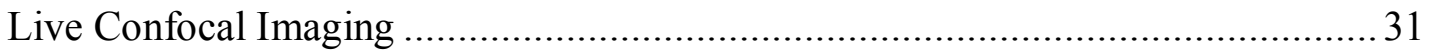

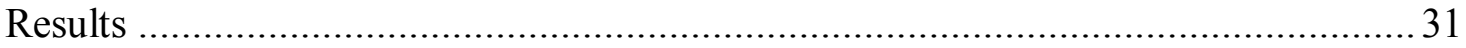

Generation and Characterization of the Zebrafish CP Enhancer Trap Line

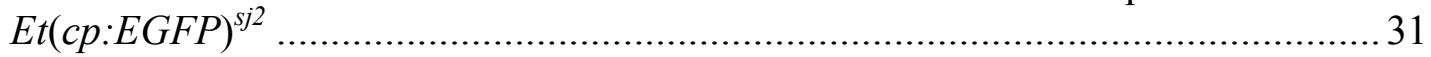

Zebrafish CP Expresses the Tight-Junction Claudin 5 and Zonula Occludens-1 ..... 35

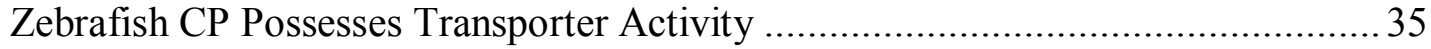

Zebrafish CP Possesses Size-Selective Barrier Properties ................................... 39

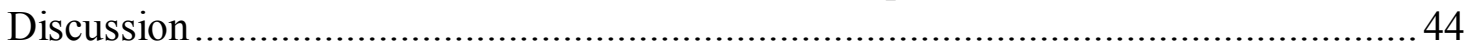

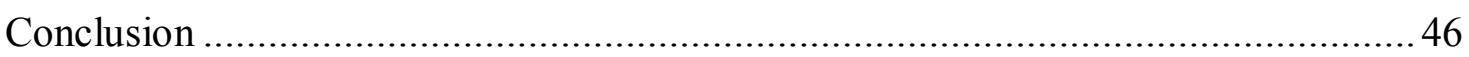




\section{CHAPTER 3. FORWARD GENETIC SCREEN IDENTIFIES CHOROID PLEXUS MUTANTS}

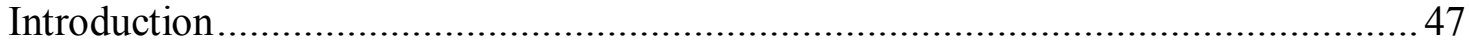

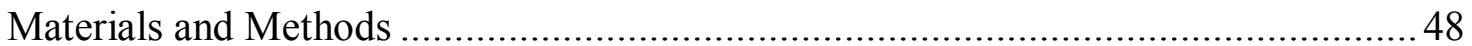

ENU Mutagenesis and Forward Genetic Screening ........................................... 48

Live Confocal Imaging and Time-Lapse Movies............................................. 49

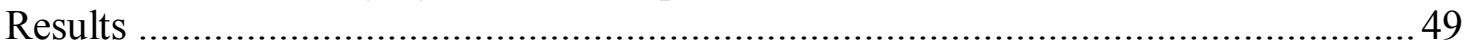

Forward Genetic Screen Identifies CP Mutants .............................................. 49

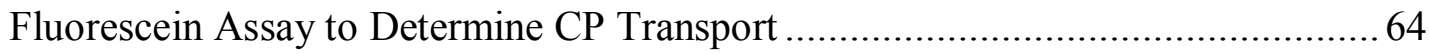

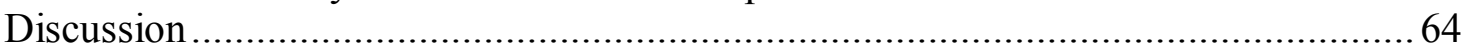

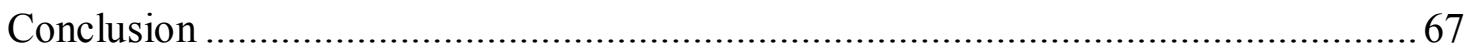

\section{CHAPTER 4. POSITIONAL CLONING AND WHOLE-EXOME SEQUENCING (WES) IDENTIFIES SART1 AS DEFECTIVE GENE IN CHOROID PLEXUS MUTANT ........................................................................68}

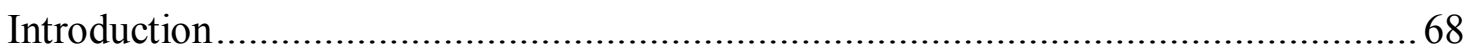

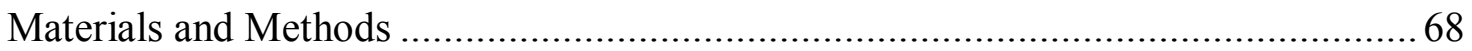

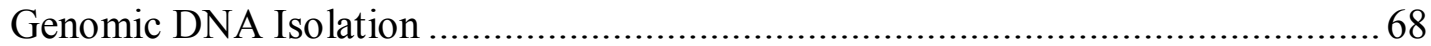

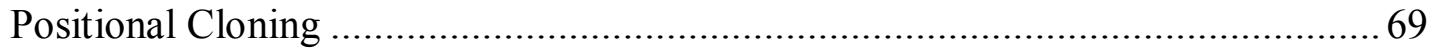

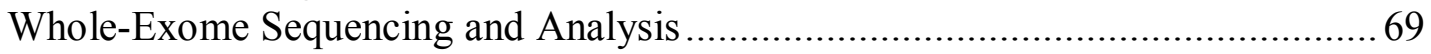

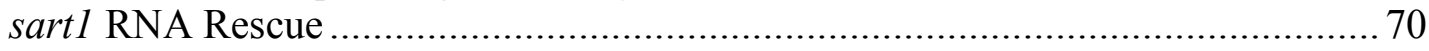

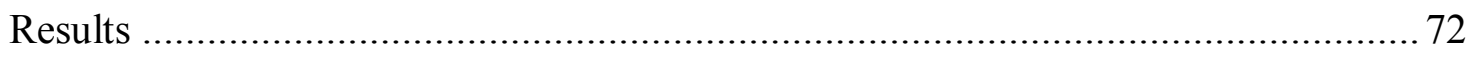

Positional Cloning and Whole-Exome Sequencing of cp27.5 Identifies Mutation

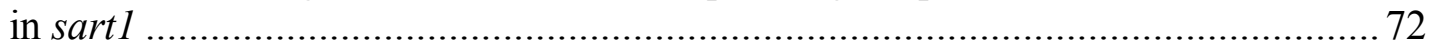

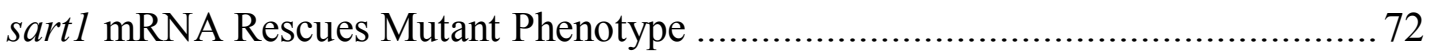

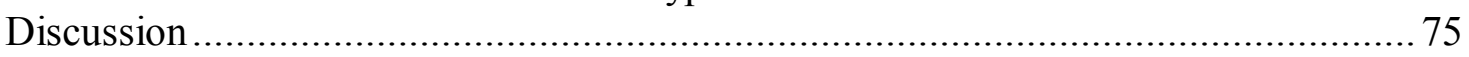

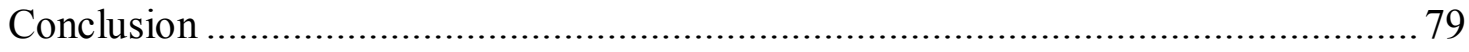

\section{CHAPTER 5. CHARACTERIZATION OF SART1 MUTANT REVEALS \\ UP-REGULATION OF GENES INVOLVED IN APOPTOSIS AND \\ DOWN-REGULATION OF VISION-RELATED GENES ....................................80}

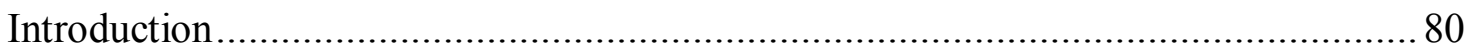

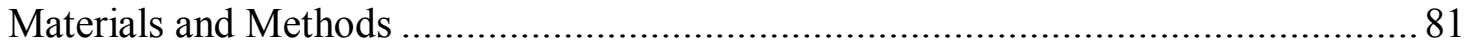

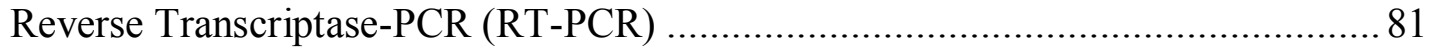

Whole Mount In Situ Hybridization ................................................................... 81

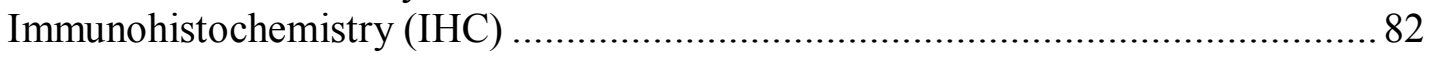

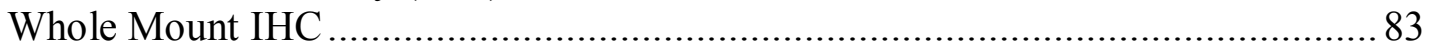

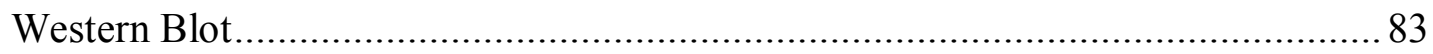

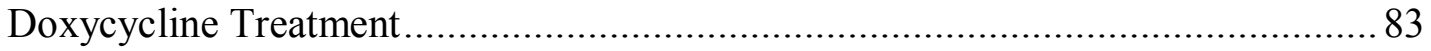

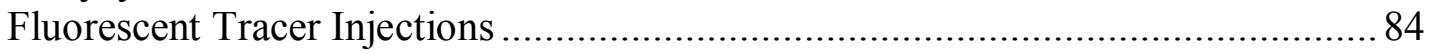

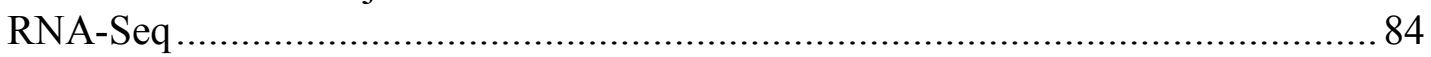

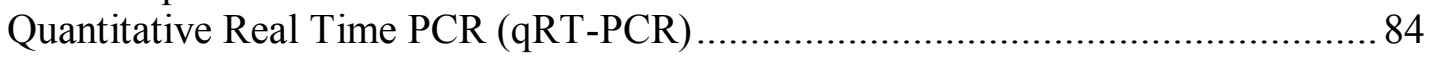

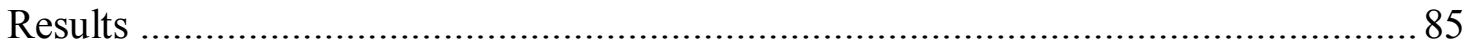

sart1 mRNA Is Maternally Derived and Expressed in the Zebrafish Brain ............ 85 
sart1 Mutants Have Altered Protein Expression of Brain and Eye Markers Along

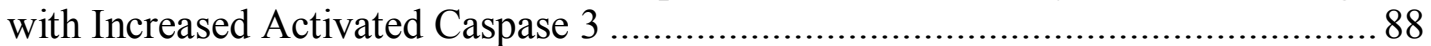

sart1 Mutants Have Increased Permeability in the Brain Ventricle .........................8 88

RNA-Seq Analysis Identifies Up-Regulated Apoptotic Genes.................................. 94

RNA-Seq Analysis Identifies Up-Regulated Spliceosome Components ................ 100

RNA-Seq Analysis Identifies Up-Regulated Claudin 5a and Matrix

Metalloproteinase Protein (MMP9) .................................................................. 100

RNA-Seq Analysis Identifies Down-Regulation of Vision-Related Genes............ 102

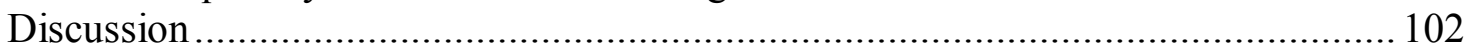

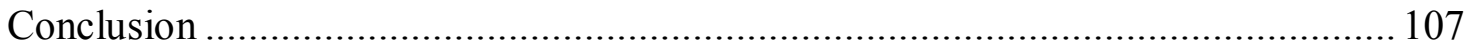

CHAPTER 6. SUMMARY AND CONCLUSIONS ..................................................... 108

LIST OF REFERENCES ...................................................................................... 110

APPENDIX A. CHAPTER 2 AND CHAPTER 3 SUPPLEMENTAL MOVIES ... 128

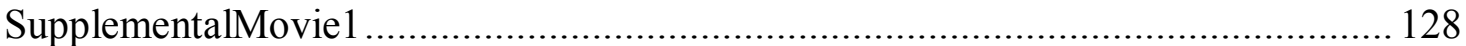

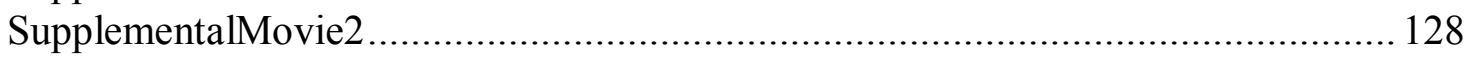

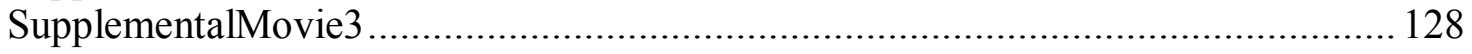

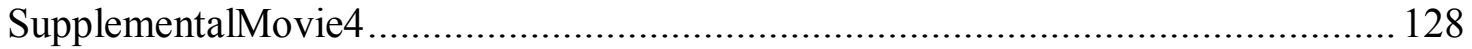

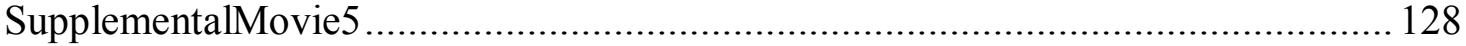

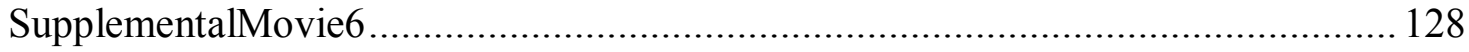

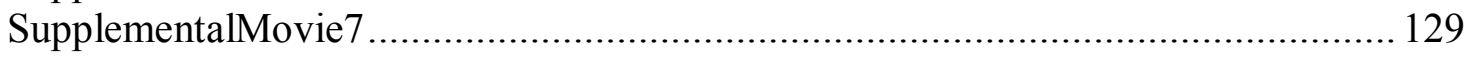

APPENDIX B. CHAPTER 5 EXTENDED METHODS AND RESULTS OF

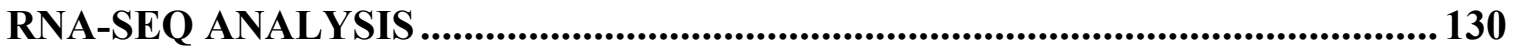

VITA 


\section{LIST OF TABLES}

Table 3-1. Number of Zebrafish Generated from the Forward Genetic Screen............50

Table 3-2. Characterization of Et(cp:EGFP $)^{s j 2}$ Mutants Generated from the Forward Genetic Screen....................................................................... 51

Table 4-1. $\quad$ sart1 mRNA Rescues Mutant Phenotypes. ........................................ 75

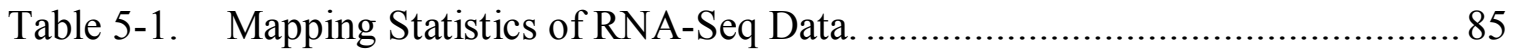

Table 5-2. Up-Regulated and Down-Regulated Genes Confirmed by qRT-PCR........ 97

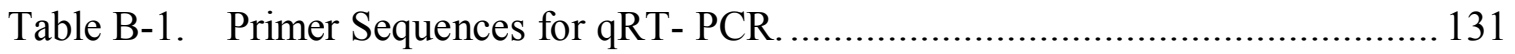

Table B-2. Top Ten Up-Regulated and Down-Regulated Genes from RNA-Seq with FPKM $>0.1$ in Both Samples. ................................................................. 132 


\section{LIST OF FIGURES}

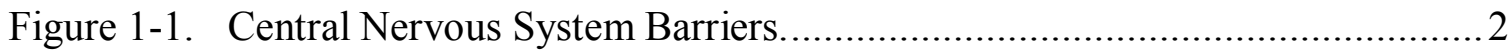

Figure 1-2. The Zebrafish Forebrain, Midbrain, and Hindbrain Ventricles Relative to

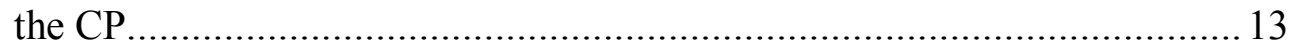

Figure 1-3. Vasculature Surrounding the CP Does Not Possess Barrier Properties....... 16

Figure 1-4. Schematic Diagram of an ENU F Forward Genetic Screen. ...................... 18

Figure 2-1. Claudin Proteins Regulate Paracellular Permeability. ...............................23

Figure 2-2. Organic Anion Transporters Control Transcellular Entry and Exit.............26

Figure 2-3. MRP1 Plays a Role in Drug Resistance at the BCSFB.............................2 27

Figure 2-4. The Et(cp:EGFP $)^{s j 2}$ Line Expresses GFP in the $\mathrm{dCP}$ and mCP. ................. 32

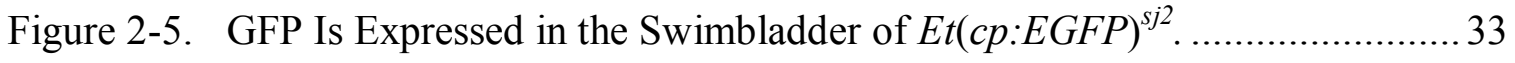

Figure 2-6. GFP Expression Is Restricted to the $\mathrm{dCP}, \mathrm{mCP}$, and Swimbladder. ............ 34

Figure 2-7. The Adult Et(cp:EGFP $)^{s j 2}$ Expresses GFP in the Saccus Dorsalis.............. 36

Figure 2-8. The Zebrafish CP Possesses the Tight Junction, Claudin 5....................... 37

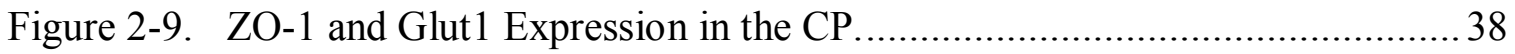

Figure 2-10. The Zebrafish CP Possesses Transporter Properties.................................... 40

Figure 2-11. Rhodamine 123 and Fluorescein Ventricle Injection Demonstrates Transporter Activity in the CP.

Figure 2-12. The Zebrafish CP Is Functional and Possesses Size-Selective Properties... 42

Figure 2-13. The Zebrafish CP Becomes Size-Selective as It Develops. ......................... 43

Figure 3-1. Spontaneous Mutants with Normal Body Morphology but

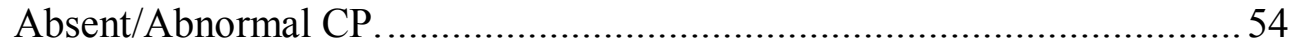

Figure 3-2. Spontaneous and Recessive Mutants with Normal CP Morphology...........55

Figure 3-3. Class I Mutants Have Reduced GFP Expression, Dispersed Epithelial

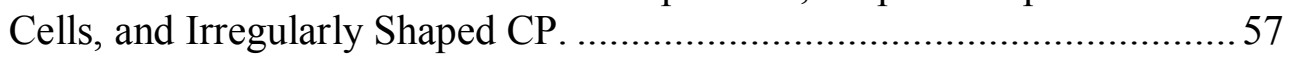

Figure 3-4. Class II Mutants Have Normal GFP Expression, Small Epithelial Aggregates, and Irregularly Shaped CP 
Figure 3-5. Class III Mutants Have Normal GFP Expression, Small Epithelial Aggregates, and Expanded CP.

Figure 3-6. Class IV Mutants Have Variable GFP Expression, Small Epithelial Aggregates, and Expanded CP.

Figure 3-7. Class V Mutants Have Normal GFP Expression, Compact Epithelial Cells, and Enlarged CP.

Figure 3-8. Fluorescein Assay Determines CP Transport Activity in Mutants. 65

Figure 4-1. Schematic of WES Sequencing Workflow and Analysis. .71

Figure 4-2. Positional Cloning Defines Critical Interval on Chromosome 21. .73

Figure 4-3. WES Identifies a Point Mutation in sart1. .74

Figure 4-4. sart1 Rescues Mutant Phenotypes. ................................................ 76

Figure 4-5. Quantification of sart1 Rescue. .77

Figure 5-1. RT-PCR and Whole Mount In Situ Hybridization Identifies sart1 Expression. 86

Figure 5-2. deltaC Expression Is Disordered in cp27.5 Mutants............................. 87

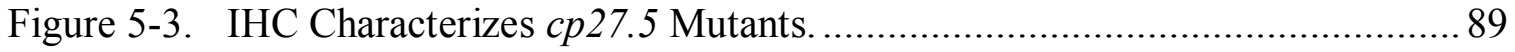

Figure 5-4. Zpr1 Is Expressed in the Pineal Gland in $c p 27.5$ Mutants...................... 91

Figure 5-5. Activated Caspase-3 Is Expressed in cp27.5 Mutant Brain. ..................... 92

Figure 5-6. Doxycycline Induces GFP in the CNS of cp27.5 Larvae.......................... 93

Figure 5-7. Fluorescent Tracer Injections Reveal Increased Permeability in Mutants. .. 95

Figure 5-8. $\quad$ qRT-PCR Confirms Up-Regulated or Down-Regulated Genes from RNA-Seq Analysis. 98

Figure 5-9. Western Blot Analysis Reveals a Larger, More Highly Expressed p53 in cp27.5 Mutant.

Figure 5-10. Claudin 5 Is Expressed in cp27.5 Mutants, but Does Not Co-Localize with Choroid Plexus Epithelia.

Figure 5-11. Schematic Diagram for the Hypothetical Role of sart1 in Apoptosis. ...... 105 


\section{LIST OF ABBREVIATIONS}

\begin{tabular}{|c|c|}
\hline $\mathrm{BBB}$ & Blood Brain Barrier \\
\hline BCSFB & Blood-Cerebrospinal Fluid Barrier \\
\hline BMP & Bone Morphogenic Protein \\
\hline Cldn & Claudin \\
\hline $\mathrm{CNS}$ & Central Nervous System \\
\hline $\mathrm{CP}$ & Choroid Plexus \\
\hline $\mathrm{CPe}$ & Choroid Plexus Epithelia \\
\hline $\operatorname{cr} x$ & cone-rod homeobox \\
\hline CSF & Cerebrospinal Fluid \\
\hline $\mathrm{Da}$ & Daltons \\
\hline DAPI & 4', 6-diamidino-2-phenylindole \\
\hline $\mathrm{dCP}$ & Diencephalic Choroid Plexus \\
\hline ECL & Extracellular Loop \\
\hline EGFP & Enhanced Green Fluorescent Protein \\
\hline EMS & Ethyl Methanesulphonate \\
\hline ENU & N-ethyl-N-nitrosourea \\
\hline FPKM & fragments per kilobase of exon per million fragments mapped \\
\hline GFP & Green Fluorescent Protein \\
\hline Glut1 & Glucose Transporter 1 \\
\hline hpf & Hours Post Fertilization \\
\hline $\mathrm{IHC}$ & Immunohistochemistry \\
\hline $\operatorname{lsm} 7$ & U6 SnRNA-Associated Sm-Like Protein \\
\hline
\end{tabular}




\begin{tabular}{|c|c|}
\hline $\mathrm{mCP}$ & Myelencephalic Choroid Plexus \\
\hline MDR & Multidrug Resistance Protein \\
\hline mRNA & Messenger RNA \\
\hline MRP1 & Multidrug Resistance-Associated Protein 1 \\
\hline NGS & Next Generation Sequencing \\
\hline OAT & Organic Anion Transporter \\
\hline PCR & Polymerase Chain Reaction \\
\hline $\operatorname{prpf31}$ & pre- $m R N A$ processing factor 31 \\
\hline qRT-PCR & quantitative real time-PCR \\
\hline RNA-Seq & RNA Sequencing \\
\hline ROI & Region of Interest \\
\hline RP & Retinitis Pigmentosa \\
\hline RT-PCR & Reverse Transcriptase-PCR \\
\hline sartl & squamous cell carcinoma antigen recognized by $T$ cells \\
\hline Shh & Sonic Hedgehog \\
\hline snRNP & Small Nuclear Ribonucleoprotein \\
\hline TER & Transepithelial Resistance \\
\hline TRE & Tetracycline Response Element \\
\hline$t t r$ & Transthyretin \\
\hline WES & Whole-Exome Sequencing \\
\hline WGS & Whole Genome Sequencing \\
\hline ZO-1 & Zonula Occludens-1 \\
\hline
\end{tabular}




\section{CHAPTER 1. INTRODUCTION}

\section{Central Nervous System Barriers}

Central nervous system (CNS) barriers serve as protective and regulatory mechanisms that allow the brain and spinal cord to remain in a homeostatic environment and function efficiently. While these structures prevent harmful components from entering CNS tissues, they can also create obstacles for efficient drug delivery. Each barrier consists primarily of tight junction and transporter proteins that function to either restrict entry into or transport components across the barrier. These proteins are localized to endothelial or epithelial cells depending on where the barrier is localized. While the focus of this study is on the choroid plexus (CP) or blood-cerebrospinal fluid barrier (BCSFB), understanding the function of other CNS barriers is essential due to their interdependence with one another in maintaining a homeostatic environment throughout the CNS. Brief descriptions of these barriers are presented in the following paragraphs.

\section{Blood-Brain Barrier}

To date, four CNS barriers have been described (Liddelow, 2011); three of which are represented in Figure 1-1. The most studied is the blood brain barrier (BBB) which is comprised mainly of endothelial cells that express tight junction and transporter proteins to create a barrier between blood and brain tissue. Additional cell types contributing to this barrier include astrocytes, pericytes, microglia, and neurons to collectively form the neurovascular unit (Abbott et al., 2010). The BBB develops by embryonic day 11 in mouse (Abbott et al., 2010) and is formed by birth (Saunders et al., 2000). Previous studies have shown that signaling mechanisms, such as the Wnt/ $\beta$-catenin pathway, are essential for BBB development and results in increased expression of the tight junction protein Claudin 3 (Liebner et al., 2008). Disruption of the $\mathrm{BBB}$ is associated with pathologies such as Alzheimer's disease, multiple sclerosis, epilepsy, and brain tumors. In Alzheimer's disease, patients have reduced cerebral blood flow and reduced glucose uptake by the brain vasculature (Bell and Zlokovic, 2009). Multiple sclerosis involves the recruitment of immune cells across the BBB into the brain resulting in CNS inflammation, while epileptic patients may exhibit a temporary opening of the BBB during an attack (Abbott et al., 2002). Utilizing the BBB as a drug delivery route has proven to be a challenge due to a number of transporters expressed on the endothelial cells that not only inhibit drug uptake, but also aid in drug resistance by pumping them out of the brain and back into circulation (Bronger et al., 2005). Future studies investigating mechanisms that restore a disrupted BBB or temporarily modulate it for drug delivery will prove to be invaluable for treating these pathologies. 


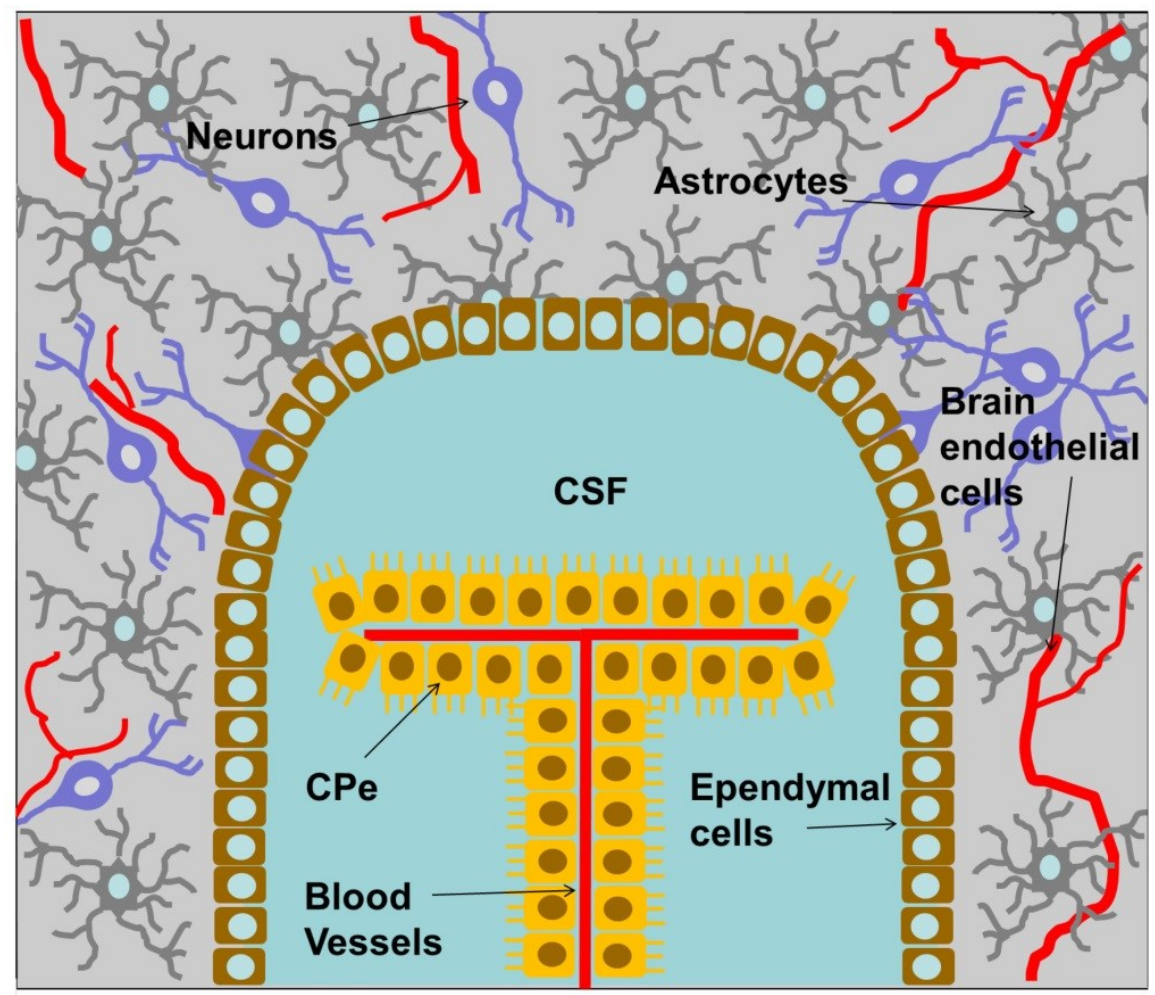

\section{Figure 1-1. Central Nervous System Barriers.}

The BBB is formed between brain endothelial cells and the surrounding brain tissue consisting of cells such as astrocytes and neurons. The ependymal layer creates a transient barrier between the CSF and the brain. The BCSFB consists of the CP epithelia $(\mathrm{CPe})$ that forms a barrier between the CSF and fenestrated blood vessels. The meningeal barrier is localized to the outer surface of the brain (not shown). 


\section{Meningeal Barrier}

Another barrier is the meningeal cover surrounding the outer surface of the brain. It consists of three layers, the dura mater, the leptomeninges containing the arachnoid membrane, and the pia mater (Decimo et al., 2012). The subarachnoid space is a region enclosing the CSF that spans between the arachnoid and pia layers. Although not as well characterized as the $\mathrm{BBB}$, it plays an important role in regulating the exchange between the blood vessels in the pia layer and the cerebrospinal fluid (CSF) localized to the subarachnoid space. The meninges originate from the neural crest cells (Mack et al., 2009). Early in development, this layer is called the inner endomeninx later forming the pia-arachnoid, and the outer ectomeninx forms the dura and cranial bones (Mack et al., 2009). The meningeal layer also plays an important role in the development of the cortex. It can secrete retinoic acid that regulates neuron production by influencing neuroepithelia to become neurons (Siegenthaler et al., 2009) and bone morphogenic protein (BMP)7 to regulate corpus callosum development in the brain (Choe et al., 2012). Subarachnoid hemorrhaging can occur in this layer. These hemorrhages can be caused by a traumatic injury or as a result of too much or too little CSF production, coagulation disorders, or vascular deformities (Mack et al., 2009). Pneumococcal meningit is is another condition commonly associated with the meninges where infections of Streptococcus pneumoniae can infect the brain and result in inflammation of the subarachnoid space (Barichello et al., 2013).

\section{Neuroependymal Cell Layer}

The neuroependyma or ependymal cells line the brain ventricles and form a barrier between the brain and CSF. However, unlike the BBB which contains tight junctions between endothelial cells, the neuroependymal cells have "strap junctions" creating a primitive barrier during development (Saunders et al., 2000). It also aids in brain formation by secreting the BMP inhibitor Noggin and promoting neural stem cells within the subventricular zone to differentiate into neurons (Lim et al., 2000). Ependymal cells arise from glial precursor cells and express glial fibrillary acidic protein (GFAP) in early development (Del Bigio, 1995). The cells possess junctions at eight weeks in humans, but still lack microvilli and cilia at this stage. Later, as cilia start to form on the apical or CSF side of the cell, they begin to move and function to transport the CSF in the direction of bulk flow (Del Bigio, 1995). These cells have also been shown to have secretory function by expressing granulophysin, a membrane protein found in transport vesicles, They also play a role in transporting and inactivating neuropeptides by expressing receptors for these molecules and producing enzymes that inhibit neuropeptide activity (Del Bigio, 1995). In addition, ependymal cells are related to diseases such as ependymomas, a type of glial cell tumor, that can arise within the spinal cord or along the brain ventricles (Hasselblatt, 2009). 


\section{Blood-Cerebrospinal Fluid Barrier}

The final barrier and the focus of this dissertation is the BCSFB. This barrier consists of the CP which contains fenestrated blood vessels and epithelial cells between blood and CSF. It is localized to the brain ventricles where CSF is stored. In mammals, there are four brain ventricles: third, fourth, and two lateral ventricles, each containing a CP. Multiple signaling pathways have been implicated in CP development such as Sonic hedgehog (Shh) (Huang et al., 2009; Nielsen and Dymecki, 2010), BMP (Currle et al., 2005), and Notch signaling (Irvin et al., 2004) which are discussed in more detail in Signaling Pathways and the Choroid Plexus on pg. 7. Similar to the BBB, the BCSFB is involved in a number of pathologies including Alzheimer's disease, multiple sclerosis (Wolburg and Paulus, 2010), and hydrocephalus (Wodarczyk et al., 2009). CP papillomas and carcinomas can also arise primarily in infants and young children (Wolburg and Paulus, 2010). How the CP contributes to these conditions is discussed in Choroid Plexus and Disease on pg. 9.

\section{Background of the Choroid Plexus}

\section{Historical Overview}

The CP was first documented by Herophilus, an ancient Greek physician, who described the CP of the lateral brain ventricles (Dohrmann, 1970). He observed that it resembled a fetal membrane or 'chorioid' resulting in its final name 'choroid plexus (Dohrmann, 1970). In 1543, the morphology of the CP in the lateral ventricles was described in De Fabrica by Vesalius (Dohrmann, 1970). The CP of the fourth and third brain ventricles were later recognized in 1664 by Willis and in 1695 by Ridley, respectively (Dohrmann, 1970). This structure was originally believed to house an animal's spirit, although future experiments done by Paul Ehrlich and Edwin Goldmann in the late 19th and early 20th century discovered its barrier function in regulating CNS homeostasis (Dziegielewska et al., 2001; Wolburg and Paulus, 2010). The role of the CP in CSF production was first suggested by Willis in 1664, and in 1855, Luschka developed the "vesicular theory" of the CP as a secretory organ (Mortazavi et al., 2014). These historical insights have paved the way for our current thinking of the $\mathrm{CP}$, not only as a structure responsible for CSF production, but also as a potential therapeutic agent or target in disease (see Protection and Repair of the Brain on pg. 5).

\section{Choroid Plexus Function}

CSF Production. Not only is the CP responsible for forming the BCSFB, but a major role of the $\mathrm{CP}$ is to produce and secrete the CSF. Humans can produce as much as $580 \mathrm{ml}$ of CSF per day and can take up to nine hours to replace this volume as it circulates (Oreskovic and Klarica, 2010). A number of transporters and aquaporin 
channels expressed on both apical and basal sides of the CPe release ions and water into the brain ventricle to generate the CSF (Brown et al., 2004). The apical and basal sides of the cell differ in the types of ion transporters expressed, creating an osmotic gradient that releases ions and water into the ventricular space. Examples of ion transporters expressed on the apical surface include $\mathrm{Na}^{+}-\mathrm{K}^{+}$ATPase and $\mathrm{Na}^{+}-2 \mathrm{Cl}-\mathrm{K}^{+}$cotransporters, while the basal side express $\mathrm{Na}^{+}$coupled $\mathrm{HCO} 3-$ transporters and $\mathrm{K}^{+}-\mathrm{Cl}^{-}$cotransporters (Brown et al., 2004).

CSF is produced first by a passive influx of plasma filtrate from the bloodstream into the interstitial space. Then, active transport mechanisms such as carbonic anhydrase, converts water and $\mathrm{CO}_{2}$ into $\mathrm{H}^{+}$and $\mathrm{HCO}_{3}-$, allowing for the exchange of these ions with $\mathrm{Na}^{+} / \mathrm{Cl}^{-}$transporters on the basolateral side. This influx of $\mathrm{Na}^{+} / \mathrm{Cl}^{-}$into the cell then activates transporters on the apical membrane to release $\mathrm{Na}^{+}, \mathrm{Cl}^{-}, \mathrm{HCO}_{3}{ }^{-}$and $\mathrm{K}^{+}$ions in an ATP-dependent manner. This creates an osmotic gradient which then stimulates aquaporin channels to secrete water towards the ventricular space thereby producing CSF (Sakka et al., 2011). In general, the CSF has lower concentrations of $\mathrm{K}^{+}$, compared to concentrations of $\mathrm{Na}^{+}$and $\mathrm{Cl}^{-}$(Sakka et al., 2011). In addition to ions, there are other components such as glucose and amino acids found in the CSF although at much lower concentrations compared to plasma. Overall protein in the CSF is also significantly reduced compared to the high levels of protein expressed in plasma (Brown et al., 2004). The route of CSF flow starts in the lateral ventricles, flows through the interventricular foramina into the third ventricle, through the cerebral aqueduct to the fourth ventricle, and then enters the subarachnoid space through the foramen of Magendie. Once in the subarachnoid space, CSF flows down into the spinal cord or is reabsorbed into the bloodstream (Sakka et al., 2011).

Protection and Repair of the Brain. Because the CP produces the CSF, it allows the brain to be maintained in a buoyant environment aiding in protection from injury. The CP can also expresses cytokines in situations of injury or disease that results in an inflammatory response (Dragunow, 2013). While the traditional role of the CP is to produce CSF and regulate CNS homeostasis, new insights have suggested that it may be therapeutically relevant. The $\mathrm{CP}$ has been identified as the gatekeeper for allowing immune cells such as leukocytes into the CSF which ultimately allows easier access to the brain. Specific cytokines such as interferon- $\gamma$ activate the CP (Schwartz and Baruch, 2014) and cell adhesion molecules such as vascular cell adhesion molecule-1 and the enzyme CD73 each contribute to the extravasation of immune cells across the barrier (Shechter et al., 2013). The CP has been shown to aid in an anti-inflammatory response by allowing the trafficking of monocytes into the CSF and migrating to areas of injury along the spinal cord (Shechter et al., 2013). The CP also secretes a number of growth factors, such as insulin-like growth factor-II and transforming growth factor- $\beta$ that aid in the development and repair of the brain. Studies have shown that insulin-like growth factor-II is secreted into the CSF follow damage to the brain and transforming growth factor- $\beta$ levels increase in response to brain ischemia (Chodobski and Szmydynger-Chodobska, 2001). Neuron formation is also influenced by growth factors that regulate progenitor cells and their patterning (Redzic et al., 2005). Because the CP 
secretes a number of growth factors, it has been suggested that epithelial cells transplanted into an injured brain could promote regrowth of surrounding tissue (Emerich et al., 2005; Thanos et al., 2010). In fact, studies have shown that grafted CP cells into a damaged spinal cord resulted in regeneration of nearby neurons (Ide et al., 2001). Recent studies have also investigated ways to encapsulate these cells for transplantation (Thanos et al., 2010).

Before we can better understand how the CP is deregulated or altered in disease, we need to understand the mechanisms governing its normal development and function. For example: while the recent proposal of using CPe for transplantation to aid in brain repair is promising due to its secretion of a number of growth factors (Emerich et al., 2005), a more comprehensive understanding of genetic and molecular pathways regulating this phenomenon is needed in order to prevent off target effects from this type of therapy. Conceivably, the lack of research investigating genetic components of CP development and function is not that this barrier is considered less important than the $\mathrm{BBB}$, but that the ability to efficiently study this structure in a living system is hindered due to its internal localization within the brain ventricle. While the systems discussed in Choroid Plexus Model Systems on pg. 10 provide a snapshot or reconstruction of how the $\mathrm{CP}$ develops and functions; the development of this structure has seldom been visualized in a living system nor has it been genetically dissected.

\section{Choroid Plexus Development}

The CP develops starting at six weeks gestation in humans and embryonic day 11 in mice (Bill et al., 2008). In humans, the stages of CP development have been classified by the amount of glycogen present in the cells, which may serve a nutritive role in the developing brain (Dohrmann, 1970). Glycogen is absent during the initial and final stages, but is abundant in CPe during intermediate periods (Dziegielewska et al., 2001). Glycogen accumulation also seems to correspond to periods of cell division within the epithelia (Sturrock, 1979). Historical electron microscopy studies have identified two types of CPe termed light and dark (Sturrock, 1979). Studies have shown that both are epithelial cells, but there may be functional discrepancies between them. Dark cells have a denser cytoplasm, more ribosomes and rough endoplasmic reticulum, and have thinner, longer microvilli compared to light cells (Dohrmann, 1970; Ormandzhieva, 2010).

After neural tube closure, the neuroepithelium, from which the CP is derived, begins to migrate into the ventricles starting around seven weeks gestation (Mortazavi et al., 2014; Strazielle and Ghersi-Egea, 2000). The neuroepithelia is initially pseudostratified tall columnar epithelium at this stage. As it differentiates, it becomes cuboidal and acquires microvilli by around week 17 and maintains this morphology for 12 weeks (Strazielle and Ghersi-Egea, 2000). At around week 29, the cells can be cuboidal or squamous and have central or basal nuclei (Mortazavi et al., 2014). The CP of the fourth ventricle develops first followed by the lateral and third ventricles (Dziegielewska et al., 2001). Early in development, these structures are referred to as the diencephalic $\mathrm{CP}(\mathrm{dCP})$ of the third ventricle, telencephalic $\mathrm{CP}(\mathrm{tCP})$ of the lateral 
ventricles, and myelencephalic $\mathrm{CP}(\mathrm{mCP})$ of the fourth ventricle (Bill et al., 2008; Strazielle and Ghersi-Egea, 2000). Wnt $3 a$ is highly expressed within the developmental regions that give rise to the $\mathrm{dCP}, \mathrm{tCP}$ and $\mathrm{mCP}$, including the diencephalic dorsal midline, the cortical hem, and the rhombic lips, respectively (Louvi et al., 2007). The cortical hem arises from neuroepithelium. Wnt signaling within this region becomes up-regulated as the CP starts to develop. Studies have shown that decreased Wnt expression in the cortical hem of mutant mice results in a loss of tCP development (Grove et al., 1998). Also at the cortical hem, genes such as Hes 1, 3, and 5 and neurogenin 2 play a role in CP epithelial fate (Imayoshi et al., 2008). Studies have demonstrated that Hes genes can repress neurogenin 2 and promote CP epithelial cell fate, while neurogenin 2 can also block Hes expression and promote Cajal-Retzius cell differentiation. Cajal-Retzius cells are neurons that aid in neuronal migration and are also derived from the cortical hem (Imayoshi et al., 2008).

The tCP of the lateral ventricles has been perhaps the most widely studied in terms of its development. This may be because models used to study the $\mathrm{CP}$, such as the opossum Monodelphis domestica, possess lateral ventricles that develop postnatally (Liddelow et al., 2010). A previous study demonstrated by injecting bromodeoxyuridine (BrdU) into developing $M$. domestica, that the tCP grows from the root outward toward the tip of the $\mathrm{CP}$ and originates from the base of the ventricular wall where neuroependymal cells are present (Liddelow et al., 2010). They observed that the number of CPe increase dramatically in early postnatal stages around P3-P5, but that this rate begins to decrease after these time points. Another interesting finding is that although neuroepdendyma continue migrating outwards as new cells are added, they no longer undergo mitosis after they have differentiated into CPe (Liddelow et al., 2010).

Studies performed in $M$. domestica demonstrate that even in early development, the CP is functional (Liddelow et al., 2009). By injecting different molecular weight dextrans either into the peritoneal cavity or CSF at early stages, size-selectivity was observed regarding passive permeability across the BCSFB in both directions (blood to CSF and CSF to blood). Protein concentration within the CSF is significantly higher in early development than in adult stages with many proteins such as albumin and transferrin that are believed to aid in brain development (Gato et al., 2004). While it might seem reasonable to assume the barrier is not formed at this stage, therefore allowing larger molecules such as proteins to penetrate the barrier, studies have demonstrated that tight junctions are already present at these early stages (Liddelow et al., 2011). By injecting bovine fetuin, the protein was observed to be transported across the barrier by only a specific subset of cells in the CP and is transferred in a unidirectional manner; it cannot be transported from the CSF into the bloodstream, but only from blood into CSF (Liddelow et al., 2009).

\section{Signaling Pathways and the Choroid Plexus}

Developmental signaling pathways such as Shh (Huang et al., 2009; Nielsen and Dymecki, 2010), BMP (Currle et al., 2005), and Notch (Irvin et al., 2004) have been 
found to contribute to CP formation. Shh is a morphogen involved in many aspects of dorsal-ventral patterning and its signaling promotes proliferation of precursor cells in the cerebellum (Huang et al., 2009). Recent evidence has shown that Shh also plays a role in the outgrowth of the $\mathrm{CP}$ into the brain ventricle and is highly expressed with the $\mathrm{CPe}$ themselves (Huang et al., 2009; Nielsen and Dymecki, 2010). These studies showed that Shh secreted by CPe can act on nearby germinal zones termed the lower rhombic lip which houses CP progenitor cells (Nielsen and Dymecki, 2010). Shh promotes proliferation of these cells to form the mature CP. Studies have also demonstrated that Shh secreted by CPe can act on nearby pericytes that help coordinate the outgrowth of the fenestrated vasculature associated with the BCSFB (Nielsen and Dymecki, 2010).

While Shh is the major contributor to ventral development, BMPs are the primary factors associated in dorsal patterning. BMP expression localized to the dorsal roof plate is present within the embryonic dorsal midline, an embryonic patterning center involved in CP development (Currle et al., 2005). BMPs can activate or repress transcription factors Msxl or $L h x 2$ respectively to specify CP development (Imayoshi et al., 2008). In vitro studies have shown that BMP4 alone can influence neuroepithelial progenitor cells to differentiate into CPe (Watanabe et al., 2012). Other BMPs such as BMP12, also known as Gdf7, have been demonstrated in fate map studies to be activated within neuroepithelial cells of the dorsal midline which later differentiate into the $\mathrm{CP}$. These studies demonstrate that removing Gdf7 before the $\mathrm{CP}$ is induced results in no $\mathrm{CP}$ development. Ablating Gdf7 later in development once the $\mathrm{CP}$ has been induced to form from neuroepithelia results in a reduced $\mathrm{CP}$ except for the posterior region of the tCP (Currle et al., 2005).

$\mathrm{CP}$ development and maintenance is also regulated by Notch signaling. Notch has been shown to be involved in organizing roof plate signaling where the dorsal midline is localized and organizing interactions between the roof plate and neural cells in the rhombic lip (Broom et al., 2012). This organizing region also signals for the roof plate to induce the expression of a common marker of the CP, transthyretin (ttr), a thyroid hormone binding protein (Broom et al., 2012). Additional studies have shown that the membrane bound Notch ligand, delta-like 3 has overlapping expression patterns with $t$ tr in the $\mathrm{CP}$ up until around embryonic day 12.5 and that mutated delta-like 3 results in a smaller CP of the fourth ventricle (Sparrow et al., 2002). Notch2 expression has been identified within CPe and Jagged1, a ligand of Notch signaling, is localized to $\mathrm{CP}$ vasculature. Similarly, as described for Shh signaling, it has been suggested that Notch 2 may also interact with Jagged 1 at the vasculature to coordinate CP development (Irvin et al., 2004). Notch2 is also localized to the microvilli of CPe and its inhibition results in microvilli malformation suggesting an addition role of Notch2 in CP maintenance (Tanaka et al., 2007).

Growth factors also influence $\mathrm{CP}$ homeostasis. Members of fibroblast growth factor receptor (FgfR) family are expressed in $\mathrm{CP}$ and one ligand, $\mathrm{Fgf2}$, is expressed in $\mathrm{CPe}$ in early development and adult stages (Greenwood et al., 2008). Evidence has demonstrated that Fgf2 is not implicated in CPe proliferation (Greenwood et al., 2008), but appears to be involved in regulating CSF production. Exogenous Fgf2 results in 
more epithelia possessing properties of dense, dark cells as described in Choroid Plexus

Development on pg. 6 with reduced secretory function (Gonzalez et al., 2010).

While the role of $W n t 3 a$ was discussed previously in Choroid Plexus

Development, other members of the Wnt pathway also act as a patterning cues for CP development including $W n t 5 a, 7 a$, and $2 b$, expressed in the telencephalon within the organizing cortical hem (Grove et al., 1998). In early development, Wnt-1 is involved along with the transcription factor Otx2 in patterning the midbrain-hindbrain boundary of the neural tube. These two genes overlap in their expression, but Wnt-1 extends in the caudal direction and Otx2 is more rostral (Bally-Cuif et al., 1995). Recently, Otx2 has been shown to be essential not only in early $\mathrm{CP}$ development, but also in maintenance. A loss of Otx2 in mice at embryonic day 9 resulted in a loss of CP formation in all brain ventricles. However, a loss of Otx2 later in development caused only the CP of the fourth ventricle to have a smaller size, whereas the CP of the lateral and third ventricles was not affected (Johansson et al., 2013). While multiple signaling pathways, such as those presented here, have shown to be essential for CP development and function, how these pathways interact with one another to generate the $\mathrm{CP}$ and downstream components involved in these pathways warrants further investigation.

\section{Choroid Plexus and Disease}

A number of diseases and conditions are associated with the CP including CP carcinomas and papillomas (Wolburg and Paulus, 2010), hydrocephalus (Wodarczyk et al., 2009), epilepsy (Suzuki et al., 2008), multiple sclerosis (Vercellino et al., 2008), and Alzheimer's disease (Alvira-Botero and Carro, 2010). Recent studies have shed light on the fact that the $\mathrm{CP}$ undergoes anatomical and physiological changes when these conditions are present. For example, disruption of the BCSFB in multiple sclerosis results in inflammation and leakage of T lymphocytes into the CSF (Vercellino et al., 2008; Wolburg and Paulus, 2010), and in Alzheimer's disease patients, the CP has decreased function in amyloid beta plaque clearance (Alvira-Botero and Carro, 2010; Wolburg and Paulus, 2010). For an extensive list of these pathologies and their association with the CP please see Emerich et al. (Emerich et al., 2005). There are also genetic variations that occur in the $\mathrm{CP}$ with these conditions, although not much is known regarding the mechanism of these genetic pathways and how they become disrupted in disease. A few examples include: myoclonin1 protein which is developmentally expressed in the CP and is mutated in epileptic patients(Suzuki et al., 2008), Twist-related protein-1 and constitutively active Notch 3 which promote invasion and cell proliferation in CP tumors (Wolburg and Paulus, 2010), and Polycystin-1 deficiency that results in hydrocephaly (Wodarczyk et al., 2009). A loss of genes in the CPe such as $p 73$, FoxJ1, and E2f5 can also result in hydrocephalus (Greenwood et al., 2008). The CP's role in regulating protein composition in the CSF is crucial in maintaining CNS homeostasis. An increase of protein in the CSF has been correlated with conditions such as schizophrenia, stroke, and depression (Liddelow et al., 2011). 


\section{Choroid Plexus Model Systems}

Previous studies have used a variety of model systems to gain insights into CP formation and function. In vitro cell culture models are used typically to investigate transport mechanisms across the barrier or to measure transepithelial resistance (TER). Studies have shown that these cultured cells mimic what is found in vivo by possessing microvilli on the apical surface, expressing tight junction proteins, and demonstrating basolateral to apical transport across the membrane (Angelow et al., 2004).

Double-chamber culture models provide a more physiological environment containing blood, epithelial, and CSF layers (Monnot and Zheng, 2013; Spuch and Carro, 2011). In addition, whole-tissue experiments have been done by dissecting the $\mathrm{CP}$ from rat or dogfish shark and maintaining the tissue in a CSF-like media (Baehr et al., 2006; Reichel et al., 2008; Villalobos et al., 2002). By incubating the intact CP with fluorescent organic ions, such as fluorescein(Villalobos et al., 2002) and Texas Red(Reichel et al., 2008), these studies have been able to uncover the mechanisms involved in the transport of organic ions; for example, whether it is active or passive and whether it is $\mathrm{Na}^{+}$or $\mathrm{K}^{+}$- dependent (Baehr et al., 2006). While in vitro systems provide benefits for understanding the CP's role at the cellular level, they do not provide a suitable developmental model to study how epithelial cells migrate over time to form the mature $\mathrm{CP}$ and do not allow for a comprehensive genetic analysis. In addition, as other studies have shown, other cell types such as pericytes or fenestrated capillaries can influence or respond to signals, such as Shh secreted by $\mathrm{CPe}$, indicating the importance of maintaining the microenvironment in these studies (Nielsen and Dymecki, 2010).

Regarding in vivo models, there have been a variety of organisms used to analyze the $\mathrm{CP}$ at various stages of life. Sheep, because of their significant CSF volume within the brain ventricles, have been used to study CSF composition and evaluate how fluid production and secretion change during aging (Chen et al., 2012; Chen et al., 2010). This is relevant for understanding the CP's role in age-related diseases such as Alzheimer's disease, where the $\mathrm{CP}$ has been shown to function in beta-amyloid clearance ( $\mathrm{Gu}$ et al., 2014). On the other hand, early CP development and size-selective barrier properties have been investigated using marsupials such as the opossum (Ek et al., 2003; Liddelow et al., 2009; Liddelow et al., 2010). When opossum pups are born, their brain is more immature compared to other species with the CP just beginning to form, therefore providing an advantage in studying early developmental processes (Ek et al., 2003; Li et al., 1997; Liddelow et al., 2010). Previous studies using this model have provided significant insight into the mechanisms regarding CP formation such as how epithelial cells present in early development still reside in later stages suggesting that the CP is not a structure with high rates of proliferation (Liddelow et al., 2010). Marsupials have also been used to determine the transport of specific proteins across the barrier such as albumin (Knott et al., 1997) and transthyretin (Li et al., 1997).

Mice have provided a genetic model for investigating genes and signaling pathways important for $\mathrm{CP}$ development and maintenance. A transcriptome study done in mice revealed that the $\mathrm{CP}$ expresses tight junctions, transporters, and neurotransmitter receptors. Some of the more highly expressed genes were transthyretin, transferrin, and 
genes involved in energy metabolism (Marques et al., 2011). The study also showed that while the $\mathrm{CP}$ has a unique transcriptome signature, it most closely resembles the $\mathrm{BBB}$ (Marques et al., 2011). Other transcriptome studies have demonstrated that the human and mouse $\mathrm{CP}$ are very similar in gene expression suggesting that they may also function in a similar manner (Janssen et al., 2013). Recent microarray data comparing adult human and mouse CP showed that there are no genes in human that are not expressed mice, although a few genes in mice are not in human (Janssen et al., 2013). The major difference between species in regard to $\mathrm{CP}$ function was that in mice, the $\mathrm{CP}$ is also responsible for carbohydrate metabolism and has a more significant role in development of the brain and eye (Janssen et al., 2013). Also, a transcriptome analysis of the CP in early development compared to adults has shown that the expression in a large number of genes do not vary between ages suggesting that even early on, the $\mathrm{CP}$ is a functional organ (Liddelow et al., 2012).

Studies of the CP in teleosts are far fewer compared to the other models mentioned previously. Teleosts have two identified CP structures, one in the third and one in the fourth ventricle (Garcia-Lecea et al., 2008). Historically, the CP of the third ventricle is often referred to as the dorsal sac or saccus dorsalis (Obermüller-Wilén, 1973). In the 1970s, comparative anatomical electron microscopy studies done in the teleost Leuciscus rutilus demonstrated that the dorsal sac and the hindbrain $\mathrm{CP}$ in the fourth ventricle are similar with one another, and also resemble the $\mathrm{CP}$ in mammals (Obermüller-Wilén, 1973). These studies found tight junction proteins and microvilli within the epithelia, and identified coated vesicles for transporting proteins (Obermüller-Wilén, 1973). While the models discussed in the preceding paragraphs provide a snapshot or reconstruction of how the $\mathrm{CP}$ develops and functions; the development of this structure has seldom been visualized in a living system nor has it been genetically dissected.

\section{Background of the Zebrafish Model}

We suggest zebrafish as a practical teleost model to study the $\mathrm{CP}$ because of advantages such as small size, transparent nature, rapid ex utero development, and genetic tractability (Goldsmith and Jobin, 2012). As discussed, in the following sections, they also possess CNS structures similar to mammals. Comparable to the dogfish shark (Villalobos et al., 2002), the CP in zebrafish lies over the brain ventricle providing an "inside out" model that allows for live imagining of its development in real time. Besides being an excellent developmental model system, zebrafish are also known for being genetically tractable. Forward genetic screens are commonly used in zebrafish due to easy maintenance and large numbers of offspring. In the following sections, the historical rise of zebrafish as model system, a discussion of their CNS attributes, and their use in developmental and genetic studies is described. 


\section{Historical Overview}

Zebrafish, (Danio rerio), is a relatively new vertebrate model system that has recently gained momentum in the field of development and genetics (Grunwald and Eisen, 2002; Stemple and Driever, 1996). Originating from streams and rivers in India (Arunachalam et al., 2013), zebrafish began to be used for scientific research in the 1960s when George Streisinger used them to study recessive mutations in genes responsible for vertebrate nervous system development (Grunwald and Eisen, 2002). Zebrafish possess multiple characteristics that made them an attractive model for these early developmental and genetic studies. One advantage is that zebrafish are vertebrates having CNS structures similar to mammals. They also grow rapidly outside of the mother and have a heartbeat by 24 hours post fertilization (hpf). Their generation period is around 3-4 months. Zebrafish are transparent during larval stages allowing development to be observed in real time. Because of their small size and large number of offspring, they are also ideal for high-throughput small molecule and genetic screens (Grunwald and Eisen, 2002; Stemple and Driever, 1996). In 1976, Streisinger, along with a colleague Charline Walker also developed methods for generating homozygous diploid embryos to discover recessive phenotypes. This was done by activating eggs with ultra-violet light-treated sperm, (which does not contribute genetic information), and suppressing the first mitotic cleavage in the egg (Grunwald and Eisen, 2002). These findings were published in Nature in 1981 (Streisinger et al., 1981). In addition to Streisinger, others also began using zebrafish as a model, such as Charles Kimmel who first used zebrafish to study neurons and brain structure (Grunwald and Eisen, 2002; Kimmel et al., 1991). Later in 1993, large-scale forward genetic screens for embryonic patterning and development were initiated in Tubingen, Germany by Christiane Nüsslein-Volhard and in Boston, Massachusetts by Marc Fishman (Grunwald and Eisen, 2002). It was reported that around 4,000 phenotypes were identified that were embryonic lethal. In 1996, an entire issue of Development was published covering forward genetic screens in zebrafish from Boston and Tubingen (Nusslein-Volhard, 2012). It was stated that more than 400 new genes were identified from these efforts (Nusslein-Volhard, 2012). In more recent years, the zebrafish genome has been sequenced making it easier to identify genes from these mutants.

\section{Zebrafish Central Nervous System}

Previous studies have confirmed that zebrafish possess CNS barriers, such as the blood-brain barrier (BBB) with functional properties similar to mammals, and a dCP and $\mathrm{mCP}$ that constitute the BCSFB (Bill et al., 2008; Garcia-Lecea et al., 2008; Jeong et al., 2008; Xie et al., 2010). The CSF in zebrafish is localized within the forebrain, midbrain and hindbrain ventricles. These structures are annotated in Figure 1-2. The development of the zebrafish CNS is similar to other vertebrates in that it arises from the neural plate on the dorsal region of the embryo (Wilson et al., 2002). However, the mechanism of neural tube formation differs in teleosts. The zebrafish neural plate forms a solid structure first called the neural keel and later hollows out to form a neurocoel as the cells begin to break away from the center of the tube (Blader and Strahle, 2000). This is in 

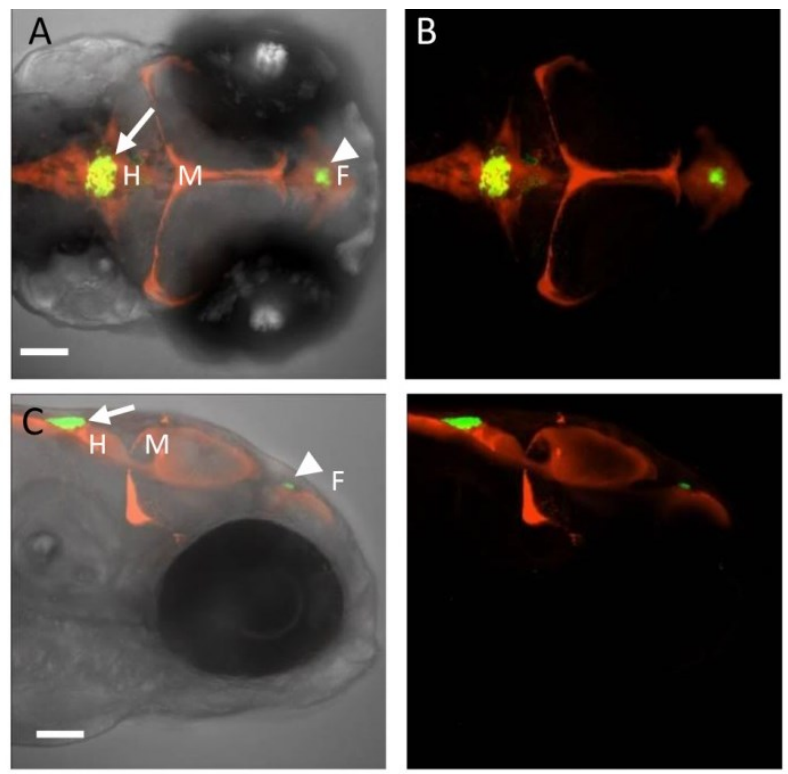

Figure 1-2. The Zebrafish Forebrain, Midbrain, and Hindbrain Ventricles Relative to the CP.

A dorsal confocal image with bright field overlay (A) and fluorescence (B) of a zebrafish at $4 \mathrm{dpf}$ with a intraventricular injection of a 2 mega-dalton (MD) rhodamine dextran (red). The yellow overlay is due to GFP expression in the $\mathrm{dCP}$ and $\mathrm{mCP}$ and the rhodamine dextran injection. (C) and (D) are similar images as (A) and (B) except from a lateral view. H: Hindbrain, M: Midbrain, F: Forebrain. Arrowhead: dCP, Arrows: $\mathrm{mCP}$. Scalebars are $50 \mu \mathrm{m}$. 
contrast to other vertebrates where neural folds form at the lateral edge of the neural plate that later fuse to form the neural tube. Signals such as BMP have also been demonstrated to be essential for neuroectoderm differentiation in zebrafish. BMPs induce the ectoderm towards an epithelial fate rather than neuronal fate. Zebrafish mutants such as snailhouse, which have a mutation in BMP7 and develop a larger neural plate due to defective BMP signaling (Blader and Strahle, 2000). Shh signaling which is secreted by the notochord underlying the neural tube plays a role in patterning ventral neurons. In specific mutants for Shh in zebrafish, the motor neurons continue to develop normally, but lateral floorplate cells are absent. Mutations in other components of the zebrafish pathway such as Gli2, a downstream component of Shh, do not affect floorplate development. This differs from mice with mutant Gli2, which do not develop a floorplate at all (Blader and Strahle, 2000). The differences in these mutants across species may be due to the genome duplication event in zebrafish where additional genes may compensate for another gene's loss of function. The zebrafish spinal cord is similar to other vertebrates with motor neurons localized to ventral regions and sensory neurons arise from the roof plate (Blader and Strahle, 2000).

Once the neural tube has formed, cells begin to divide asymmetrically with apical cells differentiating into neurons. Basal cells in the neural tube express high levels of Notch and replace the apical cells, while the differentiated neurons possess low Notch activity (Schmidt et al., 2013). The zebrafish brain develops rapidly and by $28 \mathrm{hpf}$ resembles the brain of other vertebrate embryos (Schier et al., 1996). However, the zebrafish brain appears to be inside-out, because as the telencephalon begins to develop, it folds outward with the proliferative zones on the outer surface. In mammals, the neural tube evaginates, so that the dividing cells are more internal (Schmidt et al., 2013). The same holds true for the zebrafish ependymal cells which are found at the top and dorsolateral regions of the brain ventricle in the telencephalon (Schmidt et al., 2013). As is discussed in Zebrafish as a Model for Choroid Plexus Development on pg. 15, the $\mathrm{CP}$ is also found on the outer surface of the brain.

A number of zebrafish mutants have been discovered from large-scale forward genetic screens having defects in various aspects of brain development. These mutations affect dorsal-ventral/ anterior-posterior patterning, midbrain-hindbrain boundary formation, neurogenesis, brain ventricle inflation, and brain and notochord morphology (Schier et al., 1996). Additional mutants have also been identified for genes involved in brain ventricle formation and inflation such as $\mathrm{Na}^{+} \mathrm{K}^{+}$ATPase in the snakehead mutant revealing that circulation is required for ventricle expansion as the brain develops (Lowery and Sive, 2005).

While some aspects of brain development and morphology in zebrafish may differ compared to mammals, zebrafish do possess CNS barriers similar to other vertebrates. In recent years, a number of studies have described the zebrafish BBB demonstrating that it has barrier properties similar to those found in other vertebrates, such tight junctions and transporters. One of the earlier studies demonstrated that the zebrafish BBB expressed the tight junction Claudin-5 (Cldn5) and Zonula Occludens-1 (ZO-1) which are known markers of barrier properties (Jeong et al., 2008). Another common marker of the BBB is 
the multidrug resistance transporter-1, (MDR1). Zebrafish also have Mdr1 expression that localizes to the brain vasculature and is already present as early as $2 \mathrm{dpf}$ (Umans and Taylor, 2012). In 2010, a transgenic line was created expressing GFP in the plasma to monitor BBB permeability in zebrafish. Using this line, they found that the vasculature of the brain and eye are functional at around $3 \mathrm{dpf}$ based on the presence of GFP retained within the vasculature (Xie et al., 2010). Studies have also demonstrated that within the zebrafish, neuroepithelial cells lining the brain ventricle express Cldn5 that is essential for maintaining its barrier properties between the CSF and the brain. A loss of Cldn5 resulted in reduced ventricle expansion and increased permeability between cells (Zhang et al., 2010).

\section{Zebrafish as a Model for Choroid Plexus Development}

In recent years, there have been two studies using zebrafish to analyze $\mathrm{CP}$ development and identify signaling pathways involved in its formation (Bill et al., 2008; Garcia-Lecea et al., 2008). These two studies generated distinct enhancer trap lines expressing the green fluorescent protein (GFP) in the $\mathrm{dCP}$ and $\mathrm{mCP}$. The enhancer trap lines were generated using the Tol2 transposase system (Urasaki et al., 2006), a molecular tool commonly used in zebrafish for generating enhancer trap or transgenic lines. Typically, constructs are injected into single-celled embryos and contain a fluorescent reporter to visualize integration of the construct. Once the embryos are raised to adulthood, their offspring can be screened to look for germline transmission of the transgene.

The zebrafish enhancer trap lines, referred to as Gateways and $\mathrm{Et}^{\mathrm{Mn} 16}$, provide a first-hand look into how the CP develops over time in a living organism simply by monitoring GFP expression. In Gateways, they detected GFP as early as $24 \mathrm{hpf}$ and at 36 hpf observed a sheet of cells near the midline called the tela choroidea (TC). These cells combine to form the mCP between 72-144 hpf (Garcia-Lecea et al., 2008).

Transplantation experiments in embryos demonstrated that the CPe originated from neural crest cells of rhombomere 5 (Garcia-Lecea et al., 2008). Utilizing the transparent nature of zebrafish larvae, they were also able to visualize the surrounding vasculature as the CP develops simply by incubating the larvae in Cell Trace ${ }^{\mathrm{TM}}$ Bodipy ${ }^{\circledR}$ TR methyl ester (Garcia-Lecea et al., 2008). In our studies, we have observed by intravenously injecting fluorescent tracers into $4 \mathrm{dpf}$ larvae that the overlying vasculature around the $\mathrm{CP}$ does not possess barrier properties as observed by lack of the BBB marker, glucose transporter 1 (Glut1) within the vasculature (refer to Zebrafish CP Possesses Transporter Activity on pg. 35 and Figure 1-3). This confirms what has been found in mammals where the vasculature in the $\mathrm{CP}$ does not have barrier properties, is fenestrated, and connected by covering structures called diaphragms (Ormandzhieva, 2010). To determine the signaling pathways essential for proper CP development, previous studies on the zebrafish CP utilized transgenic lines with defects in Notch and Shh signaling and crossed them with Gateways. Using time-lapse imaging, they monitored what affects these pathways had on the developing CP. Defects in Notch signaling caused the cells to differentiate early resulting in a region of GFP- negative cells along the ventricle and the 

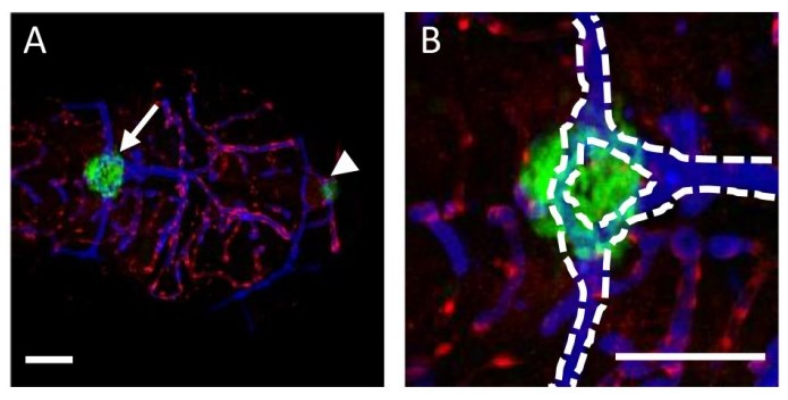

Figure 1-3. Vasculature Surrounding the CP Does Not Possess Barrier Properties.

(A) A 70 kilo-dalton $(\mathrm{kD})$ albumin tracer (blue) was injected intravenously into the $E t\left(c p: E G F P^{s j 2}\right.$ line at $4 \mathrm{dpf}$. GFP is expressed in the dCP (arrowhead) and mCP (arrow). The brain vasculature is expressing mCherry (red) under the control of the BBB maker glut1. (B) A magnified image of the $\mathrm{mCP}$ (green) and the surrounding fenestrated vasculature in blue/dashed line. BBB vasculature is shown in red. Scalebars are $50 \mu \mathrm{m}$.

GFP-positive cells forming smaller dispersed aggregates rather than one compact structure. Loss of Shh signaling resulted in fewer numbers of cells and activation of apoptosis in CPe (Garcia-Lecea et al., 2008).

A similar analysis was done using the $\mathrm{Et}^{\mathrm{Mn} 16}$ line. This line forms a rounded $\mathrm{CP}$ structure by $5 \mathrm{dpf}$. They also visualized vascular development utilizing a genetic approach by crossing it with $\operatorname{Tg}($ gatal:dsRed) and identified that the vasculature surrounding the CP originates from the dorsal longitudinal vein (Bill et al., 2008). Using a chemical approach by treating larvae with the Notch inhibitor DAPT, the CP was found to be larger overall, thereby also demonstrating the importance of Notch signaling in $\mathrm{CP}$ development. A genetic analysis looking at more specific roles of Notch signaling was done by injecting morpholinos knocking down expression of a number of Notch receptors and ligands (Bill et al., 2008).

Few studies have identified genes specifically expressed in the CP. A recent study identified a secreted protein called Clusterin specifically expressed in the CP of the developing zebrafish (Jiao et al., 2011). This localization is similar to mice in that for early development, low levels of Clusterin are detected throughout the brain, but the highest expression resides in the CP (Jiao et al., 2011). Clusterin, which aids in aggregation, has be associated with an increased risk of Alzheimer's disease a plays a role in the clearance of amyloid (A $\beta$ ) peptide (Lambert et al., 2009; Thambisetty et al., 2010). Using the Gateways transgenic line discussed previously and two-color fluorescent in situ hybridization, Clusterin expression was detected in some of the GFP-positive cells of the intact CP (Jiao et al., 2011). Another advantage of using zebrafish in this study was that they were able to answer an outstanding question of whether the role of Clusterin was regulated by IGF signaling. Because of their small size and large numbers of offspring per clutch, they aliquotted 30 to 50 zebrafish embryos per 
condition onto a 24-well plate, added an IGF-IR inhibitor to the embryo media, and determined by in situ hybridization that clusterin mRNA was not affected by IGF signaling(Jiao et al., 2011).

\section{Zebrafish Genetic Screening Strategies}

Zebrafish mutagenesis screens can be performed using a variety of approaches. The first step in performing a mutagenesis screen is treating the organism with a mutagen that will introduce mutations into the genome. Ideally, this mutation would also be introduced into the germ cells, so that it is carried throughout successive generations. A typical mutagen used in zebrafish screens is N-ethyl-N-nitrosourea (ENU) which introduces point mutations into the genome. It has been reported that ENU will introduce germ line mutations for one in every 1 x $10^{5}$ base pairs (de Bruijn et al., 2009). The efficiency of ENU mutagenesis also depends on how many rounds of treatment and how long the organism is exposed to the mutagen. Other compounds have been used such as Ethyl methanesulphonate (EMS); however the potency is not as efficient as ENU in zebrafish. Additionally, more non-specific methods can be used such as X-ray, ionizing radiation, and trimethylpsoralen; however, these can induce small to large deletions or translocations which may result in mutations in multiple genes at a specific locus (Patton and Zon, 2001).

After mutagenizing the parental generation, the next step is to determine what type of screen will be performed. Typically, in forward genetics, diploid screens are used where embryos are screened for recessive mutations in the $\mathrm{F}_{3}$ generation. This involves mating numerous siblings within $\mathrm{F}_{2}$ families until a mutation is identified in approximately $25 \%$ of the $\mathrm{F}_{3}$ offspring. This strategy is demonstrated in Figure 1-4. Another approach is to generate gynogenetic homozygous diploid embryos using either heat shock or early pressure treatment. This strategy using ultraviolet-irradiated sperm to activate the egg, does not contribute genetic information. After activation, embryos can undergo early pressure treatment where meiosis II is inhibited as the spindles are broken down and the egg retains both sister chromatids as it progresses through mitosis (Patton and Zon, 2001). Heat shock can also be performed where the first mitosis resulting in haploid embryos. The second mitosis then occurs resulting in homozygous diploids at all loci, however, these embryos have a decreased survival rate compared to those with early pressure treatment. Haploid screens are primarily used to look at early developmental stages or patterning since haploid embryos typically do not live past $3 \mathrm{dpf}$ (Patton and Zon, 2001). A limiting factor to these genetic screening strategies is that they only screen for genes that produce an identifiable phenotype.

Enhancer screens are typically performed to identify the expression patterns for classes of genes in a cell or tissue specific manner. In zebrafish, they are initiated by inserting transposons such as Tol2 or Sleeping Beauty into the genome (Korzh, 2007). Transposons are DNA fragments that can change positions within the genome. In the case of inserting a transposable element into the zebrafish DNA from another species such as Tol2 from medaka fish, an enzyme called transposase is used to allow the insert 


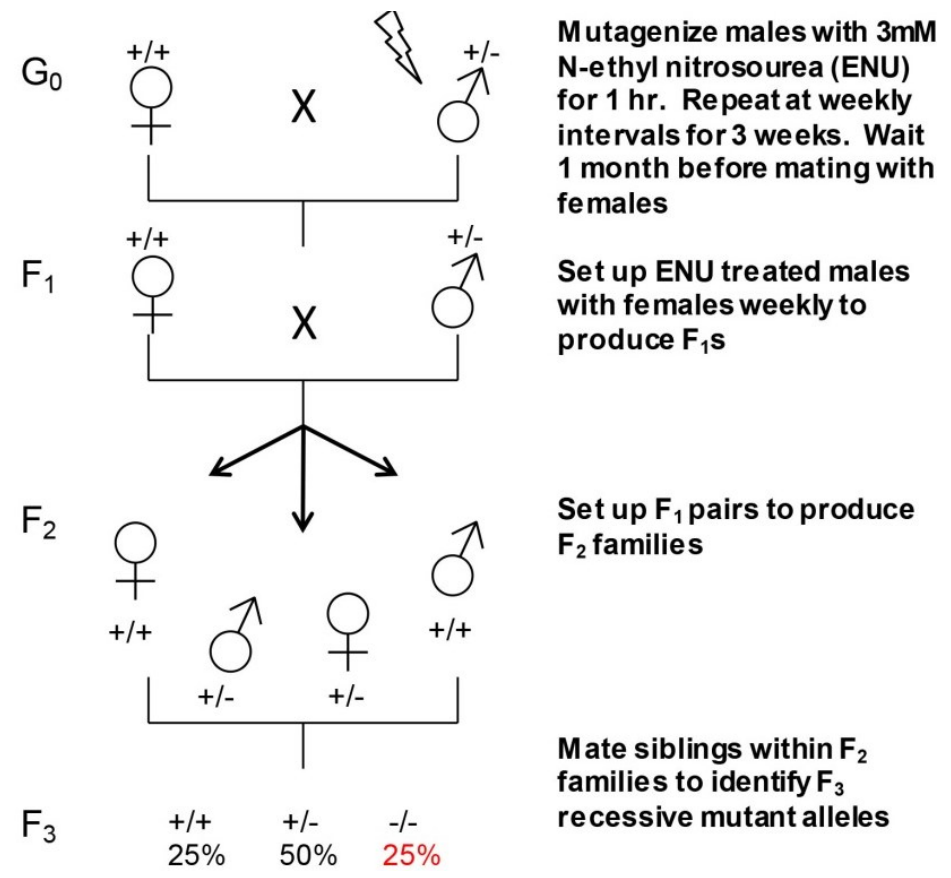

Figure 1-4. Schematic Diagram of an ENU $F_{3}$ Forward Genetic Screen. 
to jump to different regions of the genome. This strategy provides easy identification of where the transposon is inserted by using reporter genes, such as GFP, under the regulation by a weak promoter to be detected in specific regions regulated by these enhancers (Korzh, 2007).

For retroviral insertions or insertional mutagenesis screens, fragments of DNA are randomly inserted into the genome and act as a mutagen. These inserted retroviral DNAs can disrupt exons by introducing premature stop codons, or causing nonsense mediated decay (Sivasubbu et al., 2007). Insertion into an intron can result in gene silencing or alternative splicing. Another possibility is that the intron insertion has no effect on the wild-type transcript resulting in hypomorphs or only a partial loss of gene function (Sivasubbu et al., 2007). This may pose a problem for this screening method since it is reported that around $70 \%$ of retroviral insertions are in introns (Sivasubbu et al., 2007). Another obstacle in these studies has been identifying a retrovirus with strong expression (Sivasubbu et al., 2007). Nonetheless, this method has been utilized as a rapid screening strategy to identify genes involved in zebrafish embryonic development without the need for positional cloning (Allende et al., 1996; Amsterdam et al., 1997; Gaiano et al., 1996).

\section{Using Zebrafish for Genetic Analysis}

A major hurdle in forward genetics has been the time and effort it takes to map a gene, with some genes even being deemed "unmappable" due to their position along a chromosome. However, the zebrafish genome [1.5 giga bases (Gb)] has now been sequenced making gene identification much easier. Access to the zebrafish genome assemblies such as Zv8 or more recently Zv9, is also provided through database websites such as Ensembl (Flicek et al., 2014). In recent years, whole genome and exome sequencing have been utilized in zebrafish providing an alternative and more rapid way to identify the gene mutation (Kettleborough et al., 2013; Ryan et al., 2013). In the following paragraphs, traditional and innovative strategies for discovering causative genes are described along with the advantages and disadvantages of each approach.

Positional Cloning. Positional cloning is the method typically used to identify mutated genes from mutants isolated in forward genetic screens. Previous methods for detecting these genes included chromosome walking by using large genomic libraries of DNA constructs inserted into bacterial artificial chromosomes (BACs) and sequencing them to look for mutations (Zhou and Zon, 2011). Today, with the sequenced zebrafish genome, this strategy is no longer necessary. The method of positional cloning or genetic mapping is based on the process of recombination between chromosomes and identifying polymorphic regions within the genome (Zhou and Zon, 2011). Polymorphisms are regions of repetitive DNA sequences that are unique to that genome. The idea is that once the original zebrafish strain is outcrossed into a polymorphic strain (having a genetic backgrounds that differ from one another), the rate of recombination should be high in areas distant from the gene carrying the mutation and low as the distance from the mutation decreases (Zhou and Zon, 2011). This is because the mutation is only found in 
the original strain. Also, because mutants are selected based on a specific phenotype, all mutants should have the same genetic sequence at that locus. Recombination events can be analyzed by gel electrophoresis where, at the site near the mutation, the majority of mutants should only have a single band representing recombination events near zero at this location. The genes amplified in this region are said to have genetic linkage to the mutated gene (Zhou and Zon, 2011). This process has become more convenient in recent years as mapping panels have been generated containing 192 primer pairs amplifying simple sequence length polymorphisms throughout the genome (Henke et al., 2013).

After outcrossing the original line into a polymorphic strain and identifying heterozygous pairs in the next generation, the first step in positional cloning is to perform bulked segregant analysis. The goal of this step is to find a map position or the chromosome containing the mutation. This is typically done by having a pool of wild-type and a pool of mutant DNA from multiple zebrafish larvae and amplifying the DNA with polymorphic markers designed throughout the entire genome. Once a chromosome has been identified, the process of high resolution genetic mapping, using individual wild-type and mutant DNA samples and additional polymorphic markers for that chromosome, is done to define a critical interval (Zhou and Zon, 2011). Once an interval is defined containing a region of candidate genes, these genes can be sequenced to see if they contain the mutation. While this strategy does provide a way to identify the mutant gene, it is often time consuming and can be difficult to identify the gene if it is near the telomere where multiple recombination events occur.

Whole Genome and Exome Sequencing. To overcome this obstacle, new technologies using next-generation sequencing (NGS) are being used to rapidly identify genetic mutations. Whole genome sequencing (WGS) is a method that amplifies the entire genome, including non-coding regions. This approach is similar to bulked segregant analysis in positional cloning in that pools of wild-type and mutant DNA are used for sequencing. Alternatively, only mutant DNA can be used and compared to a reference genome, but this may produce false positive hits (Henke et al., 2013). After sequencing, the data is analyzed by identifying small nucleotide polymorphisms (SNPs) and screening for homozygous regions within the mutant DNA. This process of homozygosity-by-decent involves looking for regions within the genome with decreased levels of heterogeneity and reduced levels of SNPs (Bowen et al., 2012). Depending on the coverage efficiency, heterozygous regions within the genome should increase as it moves away from the mutation (Henke et al., 2013). Since most disease causing mutations occur within the coding regions of the gene and sequencing the entire genome can results in large amounts of unused data, an alternative approach is whole-exome sequencing (WES). WES, which utilizes similar strategies as WGS, provides better coverage of sequence reads by sequencing only exons within the genome. A number of genes in zebrafish mutants originating from forward genetic screens have been identified using this technique (Kettleborough et al., 2013; Ryan et al., 2013). 
RNA-Seq Analysis. A final approach in genetic mapping is RNA-Seq analysis. This technique is similar to WES sequencing in that it only sequences the coding regions or RNA and provides better coverage than WGS (Miller et al., 2013). In addition, RNA-Seq determines the amount of transcript in the sample. This technique is useful for comparing gene expression levels between wild-type and mutant and determining if other genes are affected by the mutation in the target gene (Miller et al., 2013). While RNA-Seq provides better coverage due to the smaller size of the targets, certain aspects remain that may be a hindrance for identifying the mutation such as identifying mutations affecting splice sites and the inability to detect weakly expressed transcripts (Ryan et al., 2013). Identifying the gene containing the causal mutation is similar to the previously mentioned strategies. Homozygous regions have an allele frequency of 1, with 1 being the highest frequency. The average allele frequency on the linked chromosome reaches the highest frequency at the mutated locus (Miller et al., 2013). Discovering genetic pathways critical for CP development and function and the mutations that contribute to disease is now possible due to the expansion of innovative methods utilizing in vivo model systems such as the zebrafish, and breakthroughs in genetic technologies such as WES and RNA-Seq. 


\section{CHAPTER 2. CHARACTERIZATION OF THE ZEBRAFISH CHOROID PLEXUS}

\section{Introduction}

Previous studies using zebrafish examined the involvement of Shh and Notch signaling in CP development (Bill et al., 2008; Garcia-Lecea et al., 2008). However, these studies do not analyze functional $\mathrm{CP}$ barrier properties such as tight junctions and transporters. Here, we generated an enhancer trap line, Et(cp:EGFP $)^{s / 2}$, that expresses enhanced green fluorescent protein (EGFP) specifically in epithelial cells of the dCP and $\mathrm{mCP}$. Using this enhancer trap line, we visualized its development in vivo and demonstrated that several components of the zebrafish $\mathrm{CP}$ are comparable to human and that it is functional in regulating entry into the CSF from the bloodstream. The goal of this study was to further establish zebrafish as a model to study CP development by demonstrating barrier properties similar to other vertebrates and to generate tools to characterize CP mutants identified in future studies.

\section{Central Nervous System Barrier Properties}

CNS barriers possess physical and chemical properties to regulate homeostasis within the brain. The physical barrier consists not only of the cells themselves, whether it be endothelium or epithelium, but also specialized proteins such as tight junctions and adherens junctions that substantially impede macromolecules or xenobiotics from penetrating between adjacent cells (Zlokovic, 2008). Chemical composition of the brain, blood, and CSF is regulated by numerous transporters expressed on both sides of the cell membranes that regulate the entry of hydrophilic molecules, proteins, and nutrients that cannot cross the barrier by diffusion (Zlokovic, 2008).

\section{Claudin Proteins Regulate Paracellular Transport}

The major classes of tight junctions include four-membrane spanning proteins such as claudins and occludins, and single-membrane spanning proteins such as junction adhesion molecules (JAMS) (Gunzel and Yu, 2013). Others have suggested that while each class of tight junction protein is important, Claudins appear to have the most significant role in regulating barrier permeability (Gunzel and $\mathrm{Yu}, 2013$ ). Claudins are expressed at the most apical portion of the cell and interact with Claudins on adjacent cells (Figure 2-1). In mammals there have been around 24 types of proteins identified (Krause et al., 2009). A single cell can express multiple different types of Claudins while interacting with either the same or different type of Claudin on the adjoining cell (Furuse et al., 1999).

Krause et al. (2009) describes the Claudin protein structure found in mammals. While Claudins are characterized by two extracellular loop domains (ECL1 and ECL2), 


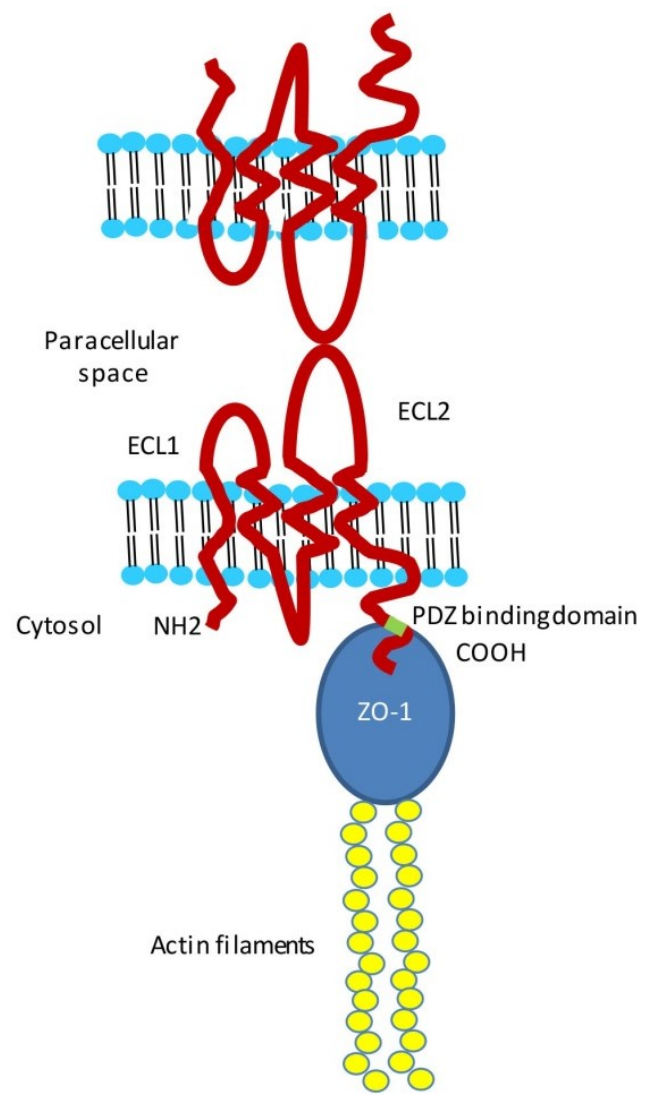

Figure 2-1. Claudin Proteins Regulate Paracellular Permeability.

Modified with permission. English, D.P., and Santin, A.D. (2013). Claudins Overexpression in Ovarian Cancer: Potential Targets for Clostridium Perfringens Enterotoxin (CPE) Based Diagnosis and Therapy. International journal of molecular sciences $14,10412-10437$. 
they also contain around seven N-terminal amino acids, and 25-55 C-terminal amino acids (Krause et al., 2009). ECL1 is primarily responsible for the barrier properties of Claudins. This loop contains two cysteines that aid in ECL1 formation and mutations of these amino acids lead to increased barrier permeability (Gunzel and $\mathrm{Yu}, 2013$ ). It is likely that these two cysteines together form disulfide bonds in the protein structure (Gunzel and Yu, 2013). ECL1 also contains positive and negative charges causing pore formation in certain types of Claudins which allows small molecules to traverse the barrier (Krause et al., 2009). Claudins can be grouped into pore-forming and barrier forming tight junctions. Pore-forming Claudins consist of Claudins 2, 7, 10, 15, and 16 while barrier-forming proteins include Claudins 1, 5, 11, and 14 (Krause et al., 2009). A combination of these two Claudin classes expressed at CNS barriers can affect TER with pore-forming Claudins decreasing TER and barrier-forming Claudins increasing TER (Anderson and Van Itallie, 2009).

A study using zebrafish to investigate Claudin 5 (Cldn5) function in brain ventricle expansion concluded that the ECL2 domain plays a role in tightening of the barrier (Zhang et al., 2010). ECL2 is responsible for forming trans Claudin-Claudin interactions (Gunzel and $\mathrm{Yu}, 2013$ ). A review by Anderson et al. proposed a two pathway model for permeability where molecules less than 4 angstroms $(\AA)$ can penetrate depending on size and charge of the Claudins expressed within a cell. This pathway is most likely regulated by ECL1. Another pathway for molecules larger than $4 \AA$ is regulated by contacts between cells and may be controlled by ECL2 (Anderson and Van Itallie, 2009). Claudins also contain a Post synaptic density protein/Drosophila disc large tumor suppressor/Zonula occuludens-1 protein (PDZ) domain at the $\mathrm{C}$ terminus. This sequence allows it to interact with PDZ domains on scaffolding proteins within the cell such ZO-1 (Zlokovic, 2008). ZO-1 interacts with filaments such as actin which then holds Claudin proteins in place within the membrane (Gunzel and Yu, 2013).

In regards to Claudin expression in CNS barriers, the BBB and BCSFB differ in the types of Claudins expressed. Claudin-5 is a tight junction largely localized to endothelial cells of the BBB. A study performed by Nitta et al. in 2003 generated Claudin-5 deficient mice which died within ten hours after birth. They discovered that a loss of Claudin-5 does not affect blood vessel morphology overall, but that in the brain, the BBB becomes more permeable to smaller molecules less than 800 daltons (Da) (Nitta et al., 2003). Zebrafish also express Cldn5 and have two paralogues, Cldn5a and 5b. Cldn5 expression has been identified in zebrafish brain endothelial cells starting at $2 \mathrm{dpf}$, but is not expressed within the tail vasculature (Xie et al., 2010). cldn5a mRNA is expressed within the midbrain, hindbrain ventricles and retina (Xie et al., 2010; Zhang et al., 2010), while $c l d n 5 b$ is expressed primarily within brain vasculature (Xie et al., 2010). Both $c l d n 5 a$ and $b$ are expressed within the hyaloid vasculature of the eye (Xie et al., 2010). Other studies in zebrafish, as described previously in Zebrafish Central Nervous System on pg. 12, have identified Cldn5a expression within the neuroepithelial cells of the developing brain that aid in ventricle expansion. Although tight junction expression has not been identified specifically in the zebrafish CP, studies in other model systems have shown that multiple Claudins are expressed within this structure such as Claudin-1, 2, 5, and 11 (Lippoldt et al., 2000; Wolburg et al., 2001). A genetic analysis in mouse 
discovered that cldn-1,3-18 and 23 were present at some level in the $\mathrm{CP}$ and that cldn2 becomes up-regulated in the adult CP (Liddelow et al., 2012). Because cldn2 is classified as a pore-forming Claudin, this may be why the $\mathrm{CP}$ is considered to be more of a secretory organ and have lower TER than the BBB, which has higher expression of the barrier-forming cldn5 (Johanson et al., 2011).

\section{Transporter Proteins Control Transcellular Permeability}

While numerous transporter families are expressed throughout the CNS, this section will focus on two types of transporters and their expression in the CP, Organic anion transporters (OATs) and MDRs. OATs, whose structure is shown in Figure 2-2, are expressed throughout the body in organs such as brain, kidney, and placenta and are responsible for the transport of small hydrophilic organic anions (Roth et al., 2012). They typically contain twelve transmembrane domains, glycosylation sites, and phosphorylation sites for kinases such as protein kinase C (PKC) (Burckhardt and Wolff, 2000) whose activation can inhibit transport activity (Sekine et al., 2006). In the CP, OAT3 is expressed on the apical cell membrane and functions to remove waste from the CSF into the bloodstream where it is ultimately excreted (Sykes et al., 2004). It is also expressed in the kidney where it transports organic anions such as para-aminohippuric acid (PAH), estrogens, and bile salts (Sykes et al., 2004). The transport of these organic anions is $\mathrm{Na}^{+}$-dependent and coupled with the transport of dicarboxylates (Sweet et al., 2003) A known substrate of OAT3 in the CP is fluorescein, a small organic green fluorescent tracer. Fluorescein appears to be specific to OAT3 transport in that null OAT3 mice have no uptake of fluorescein in the CP (Keep and Smith, 2011).

MDRs play an important role in regulating CNS homeostasis and protecting the brain from harmful xenobiotics. However, as their name implies, they are also major contributors to drug resistance. These ATP-dependent transporters contain two to three transmembrane domains and two nucleotide binding domains. The transmembrane domains play a role in recognizing and aiding in transport of hydrophilic molecules across the plasma membrane (Chang, 2003). MDRs are further classified into short and long forms. Long forms can contain as many as 17 membrane spanning domains such as Multidrug resistance-associated protein 1 (MRP1), shown in Figure 2-3, two Walker motifs that aid in ATP binding, and a conserved region called the " $\mathrm{C}$ " motif that is involved in hydrolyzing ATP. Short forms are similar except for containing only 12 membrane spanning domains (Deeley et al., 2006). Unlike OAT3 expressed on the apical membrane, MRP1 is expressed on the basolateral membrane of the CP and functions to transport substrates from CSF into the bloodstream (Keep and Smith, 2011). A widely known substrate of MRP1 is the anti-cancer drug etoposide. Studies performed in MRP1 knockout mice observed an increased accumulation of etoposide within the CSF indicating that this transporter has a significant role in drug distribution in the CNS (Wijnholds et al., 2000). 


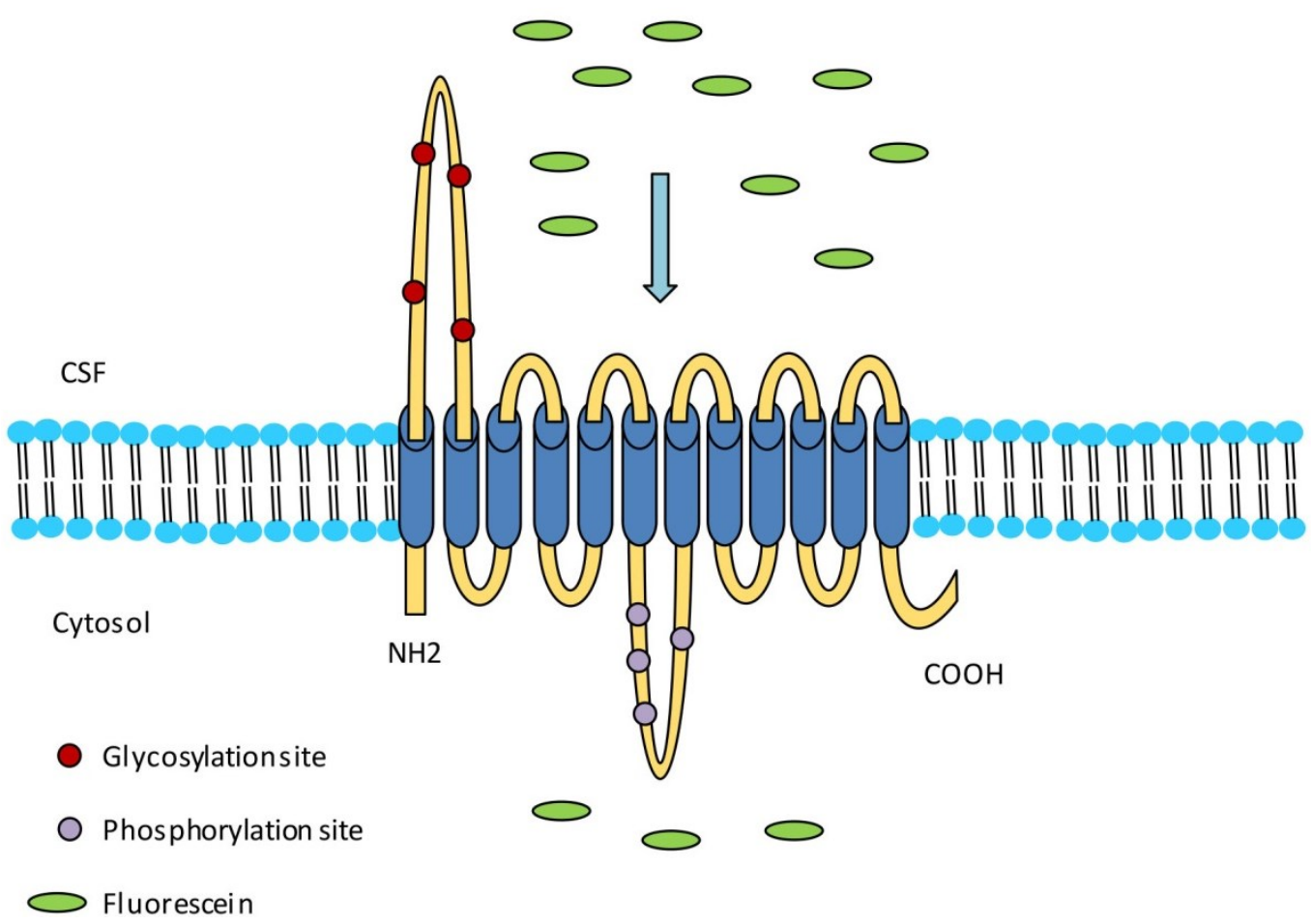

Figure 2-2. Organic Anion Transporters Control Transcellular Entry and Exit.

Modified with permission. Sweet, D.H., Bush, K.T., and Nigam, S.K. (2001). The organic anion transporter family: from physiology to ontogeny and the clinic. American journal of physiology Renal physiology 281, F197-205. 


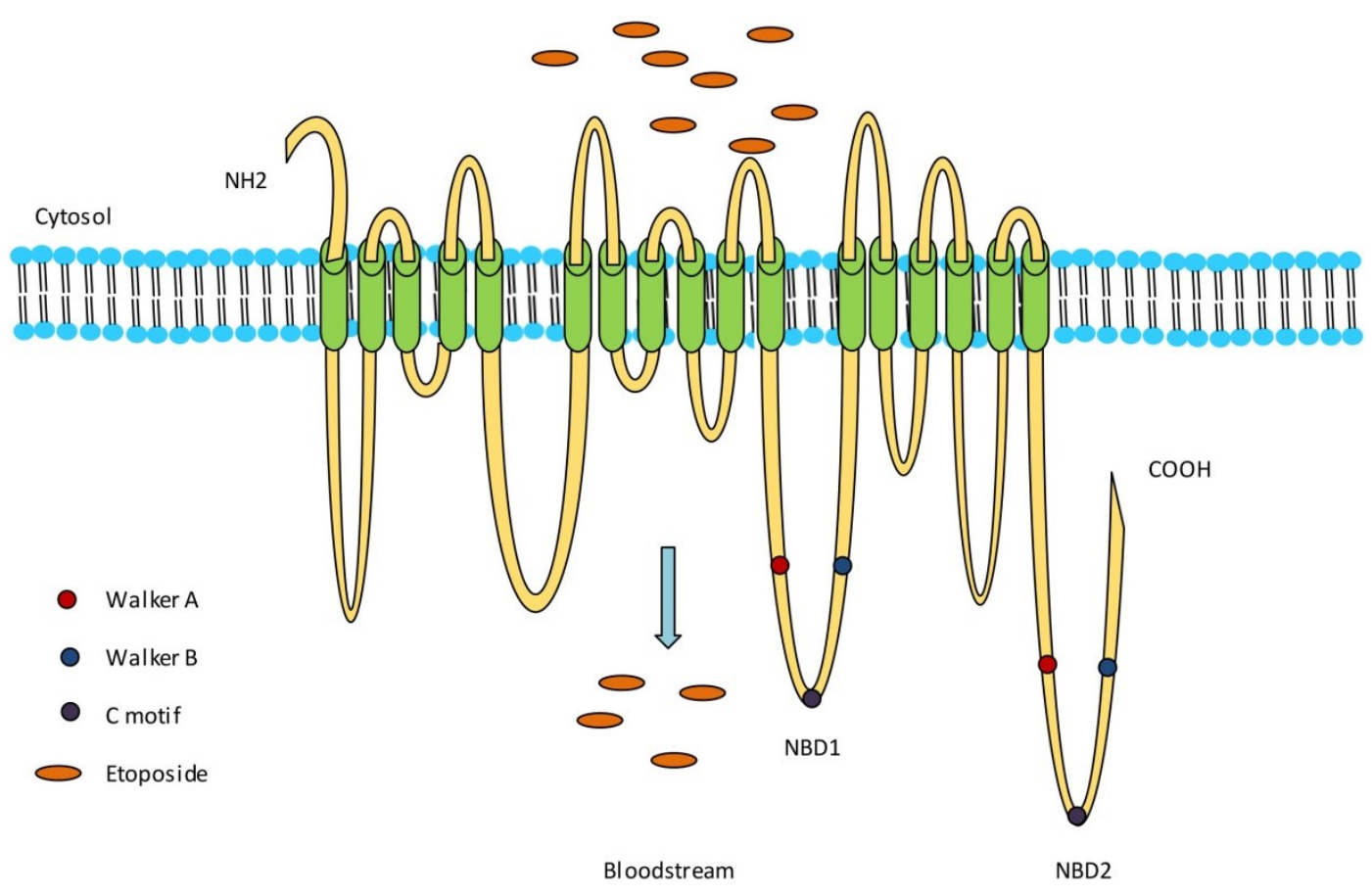

Figure 2-3. MRP1 Plays a Role in Drug Resistance at the BCSFB.

Modified with permission. Deeley, R.G., Westlake, C., and Cole, S.P. (2006).

Transmembrane transport of endo- and xenobiotics by mammalian ATP-binding cassette multidrug resistance proteins. Physiological reviews $86,849-899$. 


\section{Materials and Methods}

\section{Fish Lines and Maintenance}

Zebrafish were maintained at $28.5^{\circ}$ on a $14 \mathrm{~h}$ light/dark cycle. Embryos used for imaging or screening F3 larvae were initially collected in egg water $(0.03 \%$ Instant Ocean) containing $0.02 \%$ methylene blue and treated at $24 \mathrm{hpf}$ with $0.003 \%$ 1-phenyl-2-thiourea (PTU) (Sigma) to prevent pigment formation. Zebrafish were maintained in accordance with established protocols and regulations of the St. Jude Children's Research Hospital and Institutional Animal Care and Use Committee.

\section{Generation of the $\operatorname{Et}(c p: E G F P)^{s j 2}$ Line}

To generate the Et(cp:EGFP $)^{s j 2}$ line, EGFP was released from the pEGFP-N1 vector (Clontech) by BamHI and NotI digestion and inserted into the pTRE-Tight vector (Clontech). The Tetracycline Response Element (TRE)-tight:EGFP fragment was released by XhoI and ClaI digestion and inserted into the pT2AL200R150G vector (Urasaki et al., 2006). Approximately $30 \mathrm{pg}$ of the resulting T2 (TRE-tight:EGFP) plasmid DNA was co-injected with $30 \mathrm{pg}$ of in vitro-transcribed Tol2 transposase mRNA into single-cell embryos of the $\mathrm{AB}$ strain. The embryos were raised to adults and screened for germline transmission by examining their offspring for EGFP expression.

\section{Fluorescent Tracer Injections}

To observe transporter activity in the CP, Et (cp:EGFP) $)^{\mathrm{sj} 2}$ larvae at $4 \mathrm{dpf}$ were anesthetized in $0.02 \%$ tricaine and injected intravenously into the common cardinal vein with approximately 1-2 nL of $100 \mu \mathrm{M}$ rhodamine 123 (Sigma-Aldrich) using a micromanipulator and a pneumatic picopump (World Precision Instruments). Immediately after the injections, larvae were embedded in $1.2 \%$ low-melting-point agarose (Invitrogen) made in egg water. Larvae were imaged on a Nikon C1Si confocal microscope and analyzed using Nikon EZC1 3.91 software.

To study CP function, Casper larvae (lacking melanocytes and iridophores) (White et al., 2008) were anesthetized in $0.02 \%$ tricaine and intravenously injected into the common cardinal vein with approximately $0.3-2 \mathrm{~nL}$ of $10 \mathrm{mg} / \mathrm{mL}$ rhodamine- and fluorescein-labeled dextrans at 2, 3, and 4 dpf. Fluorescent tracers included a 3-kDa fluorescein dextran, a 10-kDa rhodamine dextran, and a 40-kDa anionic fluorescein dextran (Invitrogen). Immediately after the injections, larvae were laterally embedded in $1.2 \%$ low-melting-point agarose made in egg water. To observe tracer permeability into the brain ventricle, time-lapse images were collected every 2 min for $1 \mathrm{~h}$ with a Nikon AZ100 microscope equipped with a shutter (Sutter Instruments) and analyzed with Nikon NIS-Elements 3.2 software. Et(cp:EGFP) $)^{s j 2}$ larvae were also injected intravenously with 
the 10-kDa rhodamine dextran at 2 and $4 \mathrm{dpf}$ and imaged on a Nikon C1Si confocal microscope with Nikon EZC1 3.91 software.

To visualize brain ventricle morphology in wild-type and mutant $E t(c p: E G F P)^{s j 2}$ larvae at $4 \mathrm{dpf}$, PTU-treated zebrafish were anesthetized and intraventricularly injected with approximately $1.8 \mathrm{~nL}$ of $40-\mathrm{kDa}$ fluorescein-labeled dextran as described previously (Gutzman and Sive 2009). Larvae were imaged dorsally immediately after the injection, using a Nikon SMZ1500 epifluorescence stereoscope and Nikon NIS-Elements 3.1 software. Ventricle injections of rhodamine 123 and fluorescein were performed as described previously (Gutzman and Sive 2009) in Casper larvae at 4 dpf and imaged on a Nikon C1Si confocal microscope with Nikon EZC1 3.91 software.

\section{Fluorescein Treatment}

Casper and $E t(c p: E G F P)^{s j 2}$ larvae were incubated for $4 \mathrm{~h}$ in the dark with $50 \mu \mathrm{M}$ fluorescein (Fluka). Larvae were briefly rinsed 12 times in egg water, anesthetized in $0.02 \%$ tricaine, and embedded in 1.2\% low-melting-point agarose. After $30 \mathrm{~min}$, larvae were imaged using a Nikon SMZ1500 epifluorescence stereomicroscope and analyzed with Nikon NIS-Elements 3.1 software.

\section{Statistical Analysis of Tracer Permeability}

Three regions of interest (ROIs) with areas of $0.02 \mathrm{~mm}^{2}$ were selected in both the heart and the brain ventricle. Using the NIS-Elements 3.2 software, the mean fluorescence intensity, which served as a read-out for tracer permeability into the brain ventricle, was measured for each ROI over a $1 \mathrm{~h}$ period. Using Microsoft Excel, the average of the mean fluorescence intensity for the 3 ROIs in the heart and the 3 ROIs in the brain ventricle was calculated for each sample. An overall average was then calculated for the mean fluorescence intensity in the heart and the ventricle for all trials $(n=7)$. The dataset of the mean fluorescence intensity in the heart and ventricle was normalized to give values between 0 and 1 .

The ratio of the fluorescence intensity in the ventricle to that in the heart was calculated at the 30-min time point when the leakiest tracer reached saturation. The ratio was then normalized against the tracer having the greatest mean intensity in the ventricle at $30 \mathrm{~min}$. A one-way ANOVA test was performed in Microsoft Excel to determine whether there was a significant difference in fluorescent intensity between the tracers and developmental time points. A Tukey's post-hoc test was performed to determine which tracers were statistically significant to one another at each developmental time point. A significance value of $\alpha=0.05$ was used, and $P$ values were calculated using the GraphPad software P value calculator. Error bars were based on the mean of seven observations using standard error. 


\section{Immunohistochemistry (IHC)}

Larvae were anesthetized in $0.02 \%$ tricaine and fixed in $4 \%$ paraformaldehyde (PFA) (Electron Microscopy Sciences) at $4^{\circ}$ overnight and washed the next day in $1 \mathrm{x}$ phosphate buffered saline (PBS) (Calbiochem). Samples were sunk in 30\% sucrose/PBS at $4^{\circ}$ overnight. Larval and 1-month-old brain tissues were embedded in Optimal Cutting Temperature (O.C.T.) Compound (Tissue-Tek), frozen on dry ice, and stored at $-80^{\circ}$. Tissues were sectioned using a Leica CM 1950 cryostat. Sections were washed in PBS for 5 min followed by three 5 min washes in PBST [PBS/ 0.03\% Triton X-100 (Sigma)]. Sections were incubated in blocking buffer [PBST with 5\% goat serum (Gibco) and 1\% BSA (Sigma)] for $3 \mathrm{~h}$ at room temperature. Primary antibodies were incubated at $4^{\circ}$ overnight followed by secondary antibody incubation for $2 \mathrm{~h}$ at room temperature. Sections were washed in PBST four times for 15 min after primary and secondary antibody incubations. Primary antibodies included rabbit anti-GFP (1:500; Invitrogen), mouse anti-GFP (1:500, Life Technologies), rabbit anti-ZO-1 (1:25, Invitrogen), rabbit anti-Glut1 (1:200, Novus Biologicals), and mouse anti-Zpr1 (1:100; ZIRC). Secondary antibodies included Alexa Fluor goat anti-rabbit 488 (1:200; Invitrogen) and Alexa Fluor goat anti-mouse 555 (1:200; Invitrogen). Antibody dilutions were prepared in blocking buffer. Sections were counterstained with $1 \mu \mathrm{g} / \mathrm{mL}$ 4', 6-diamidino-2-phenylindole (DAPI) (Roche) for $1 \mathrm{~min}$, rinsed briefly in PBS, and mounted with Fluoromount (Electron Microscopy Sciences). Images were taken on a Nikon AZ100 microscope and analyzed using NIS-Elements AR 3.2 software.

\section{Whole Mount IHC}

Embryos were incubated in egg water with $0.003 \%$ PTU to prevent pigment formation. At 4 and 6 days postfertilization (dpf), larvae were anesthetized in $0.02 \%$ tricaine and fixed in 4\% PFA overnight. The next day, samples were washed in 1x PBS followed by $1 \mathrm{x}$ PBST and treated with $20 \mu \mathrm{g} / \mathrm{mL}$ Proteinase K (New England Biolabs) for $15 \mathrm{~min}$. The reaction was stopped by adding 10\% lamb serum (Gibco) followed by additional washes in PBST. Samples were blocked with 10\% lamb serum for $1-4 \mathrm{~h}$ and incubated in primary antibody at $4^{\circ}$ overnight. Samples were washed the next day in PBST and incubated in secondary antibody at $4^{\circ}$ overnight. Antibodies used included rabbit anti-GFP (1:100; Invitrogen), mouse anti-GFP (1:200; Life Technologies), rabbit anti-ZO-1 (1:50; Invitrogen), rabbit anti-Glut1 (1:100, Novus Biologicals), mouse anti-Zpr1 (1:50; ZIRC), mouse anti-Cldn5 (1:100; Invitrogen), Alexa Fluor goat anti-rabbit 488 (1:200; Invitrogen) and Alexa Fluor goat anti-mouse 555 (1:200; Invitrogen). Additional washes in PBST were done the following day and stored in $1 \mathrm{x}$ PBS. Samples were embedded in $0.8 \%$ low-melting point agarose (Invitrogen) made in 1x PBS and imaged on Nikon C1Si laser scanning confocal microscope. Z-stacks were compiled to create maximum intensity projection images using Nikon NIS-Elements 3.1 imaging software. 


\section{Live Confocal Imaging}

Confocal imaging was performed on a Nikon C1Si confocal microscope and analyzed using Nikon EZC1 3.91 software. Scans of DIC and GFP images were acquired using a $20 \mathrm{x}$ objective at $4 \mu \mathrm{m}$ intervals to create a $\mathrm{z}$-stack maximum intensity projection image. To observe CP development, larvae were collected at approximately $30 \mathrm{hpf}$ and prepared for imaging as described above. Z-stack images were acquired every $30 \mathrm{~min}$ over a 65 h period. Using NIS-Elements AR 4.0 software, images were smoothed and z-stacks were compiled to create an enhanced depth of focus (EDF) image.

\section{Results}

\section{Generation and Characterization of the Zebrafish CP Enhancer Trap Line $\operatorname{Et}(c p: E G F P)^{s j 2}$}

In the process of producing a tetracycline-inducible transgenic line, we fortuitously generated the zebrafish $\mathrm{CP}$ line by an enhancer trap effect. To generate this line, we co-injected a T2 (tetracycline response element (TRE)-EGFP) construct with Tol2 transposase mRNA into single-cell zebrafish embryos. The TRE-EGFP construct integrated into an enhancer region regulating the $\mathrm{CP}$. The insertion was transmitted to subsequent generations, thereby creating a stable line termed Et $(c p: E G F P)^{s j 2}$.

GFP expression was specifically localized to the $\mathrm{dCP}$ in the third ventricle and the $\mathrm{mCP}$ in the fourth ventricle at $3 \mathrm{dpf}$ (Figure 2-4A). Unlike mammals, the zebrafish $\mathrm{CP}$ is localized outside the brain allowing for easy visualization of CP development. As mentioned in the two previous zebrafish CP studies, the mCP in teleosts exists as a layer of GFP positive cells directly below the epithelium near the roof of the fourth ventricle and the $\mathrm{dCP}$ is at the most dorsal portion of the third ventricle (Bill et al., 2008; Garcia-Lecea et al., 2008) allowing CP development to be easily visualized in the zebrafish due to its "inside-out" morphology.

We also detected GFP expression in the heart and swimbladder. The swimbladder consists of epithelial cells and has been compared to the mammalian lung (Winata et al., 2009). GFP expression along the lining of the swimbladder is shown in Figure 2-5. Using time-lapse confocal microscopy, GFP expression was detected in a subset of dCP cells as early as $30 \mathrm{hpf}$ (see Appendix A. SupplementalMovie1). We found that the mCP epithelial cells migrated from the outer rhombomeres into the midbrain-hindbrain boundary as described previously (Garcia-Lecea et al., 2008) and also anteriorly from the developing spinal cord. These cells increase in GFP intensity as they coalesce to form a compact structure by approximately $72 \mathrm{hpf}$. There were no additional changes in CP morphology by 96 hpf. We also observed a few GFP positive spots in other areas of the head and eyes. However, after staining Et(cp:EGFP $)^{s j 2}$ larvae at $3 \mathrm{dpf}$ with a GFP antibody, we only observed labelling within the $\mathrm{dCP}, \mathrm{mCP}$, and swimbladder (Figure 2-6). 

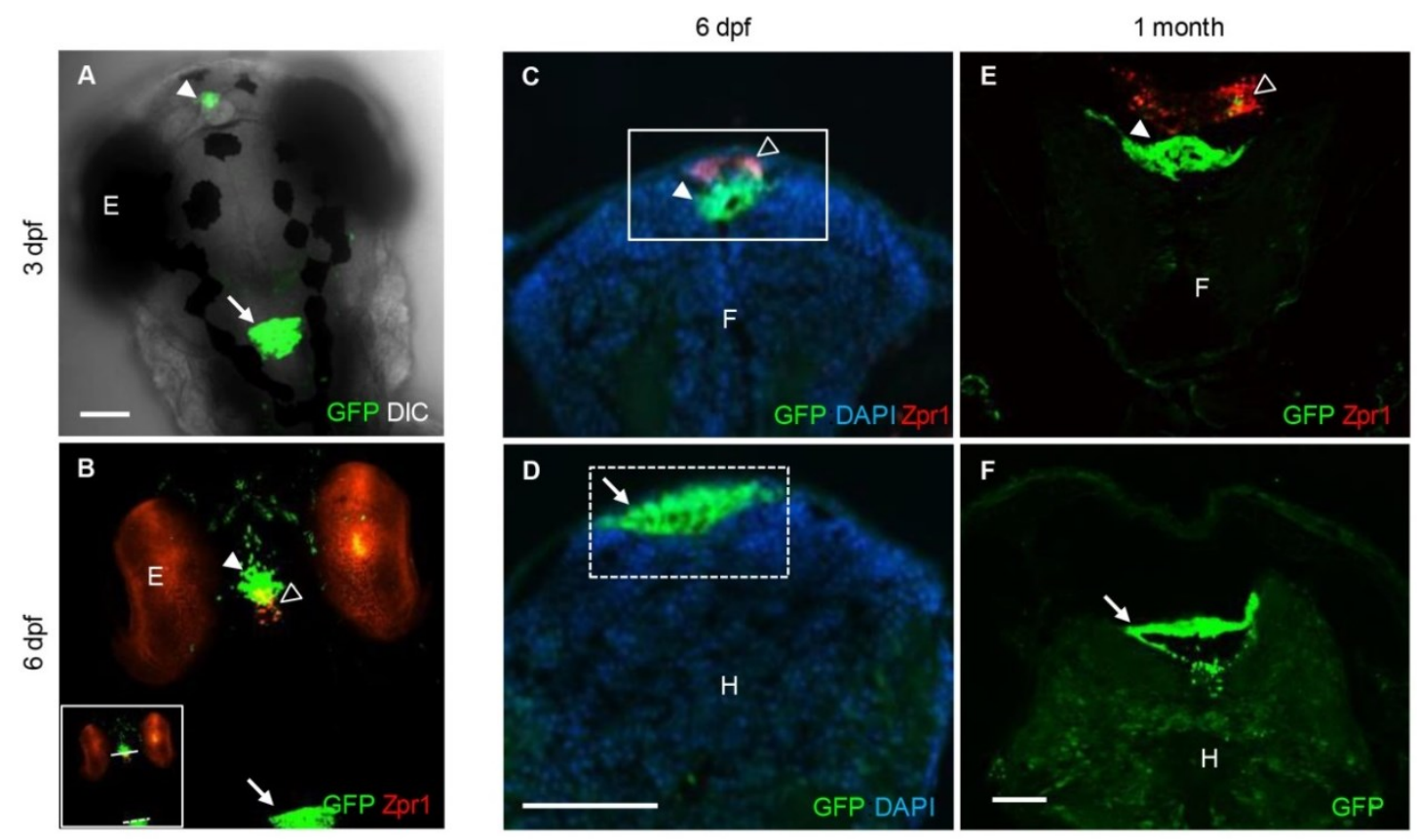

Figure 2-4. The Et(cp:EGFP $)^{s j 2}$ Line Expresses GFP in the dCP and mCP.

(A) Confocal image of the dorsal anterior view of 3 dpf $E t(c p: E G F P)^{s j 2}$ larvae expressing GFP in the $\mathrm{dCP}$ and $\mathrm{mCP}$. (B) Whole-mount immunohistochemical staining of $E t(c p: E G F P)^{s j 2}$ larvae at $6 \mathrm{dpf}$. GFP in green labels the dCP and mCP, and Zpr-1 shown in red labels the pineal gland and photoreceptors. The yellow overlay demonstrates the interaction between the pineal gland and $\mathrm{dCP}$. The solid and dashed lines across the $\mathrm{dCP}$ and $\mathrm{mCP}$ in the inset image indicate approximate regions where sections were taken for Figure 2-4C-D. (C) Transverse section through the dCP of $6 \mathrm{dpf}$ $E t(c p: E G F P)^{s j 2}$ larvae stained with GFP, DAPI, and Zpr-1. (D) Transverse section through the mCP of $6 \mathrm{dpf} E t(c p: E G F P)^{s j 2}$ larvae stained with GFP and DAPI. (E) Transverse section through the dCP of a 1-month-old Et(cp:EGFP $)^{s j 2}$ zebrafish stained with GFP and Zpr-1. (F) Transverse section through the mCP of a 1-month-old $E t(c p: E G F P)^{s j 2}$ zebrafish stained with GFP. Abbreviations: dCP, diencephalic CP; mCP, myelencephalic CP; F, forebrain; H, hindbrain; GFP, green fluorescent protein; DAPI, 4', 6-diamidino-2-phenylindole. For all images: filled arrowhead, dCP; open arrowhead, pineal gland; arrow, mCP. Scale bars are $50 \mu \mathrm{m}$. 

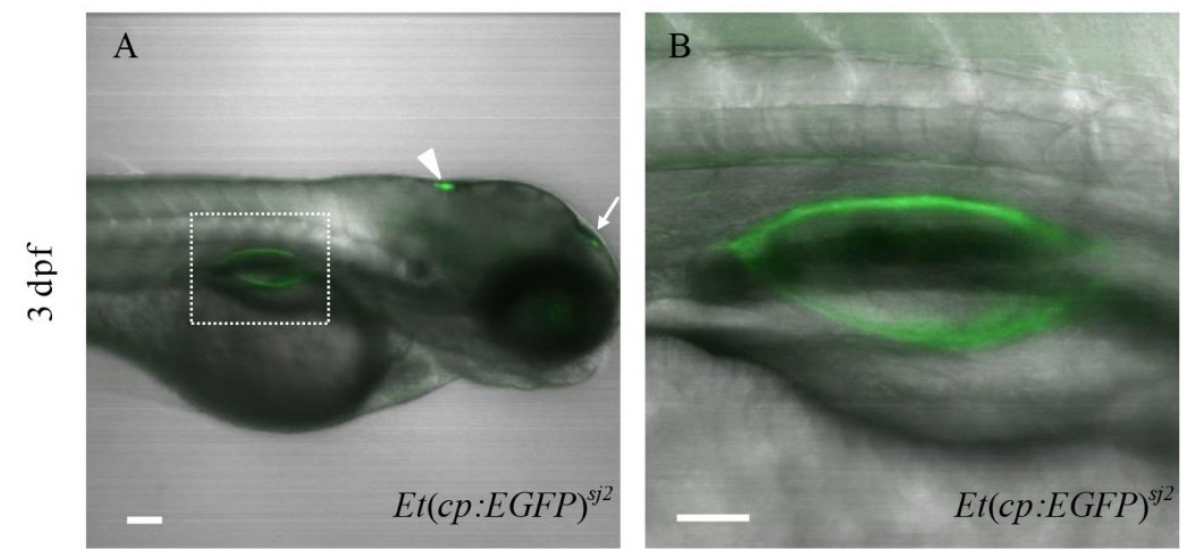

Figure 2-5. GFP Is Expressed in the Swimbladder of Et(cp:EGFP $)^{s j 2}$.

(A) $E t(c p: E G F P)^{s j 2}$ larvae at 3 dpf expresses GFP in the dCP (arrow), mCP (arrowhead), and swimbladder. Dashed box is magnified in (B) showing GFP lining the cells of the swimbladder. $\mathrm{dCP}$, diencephalic choroid plexus; $\mathrm{mCP}$, myelencephalic choroid plexus. Scale bars are $50 \mu \mathrm{m}$. 


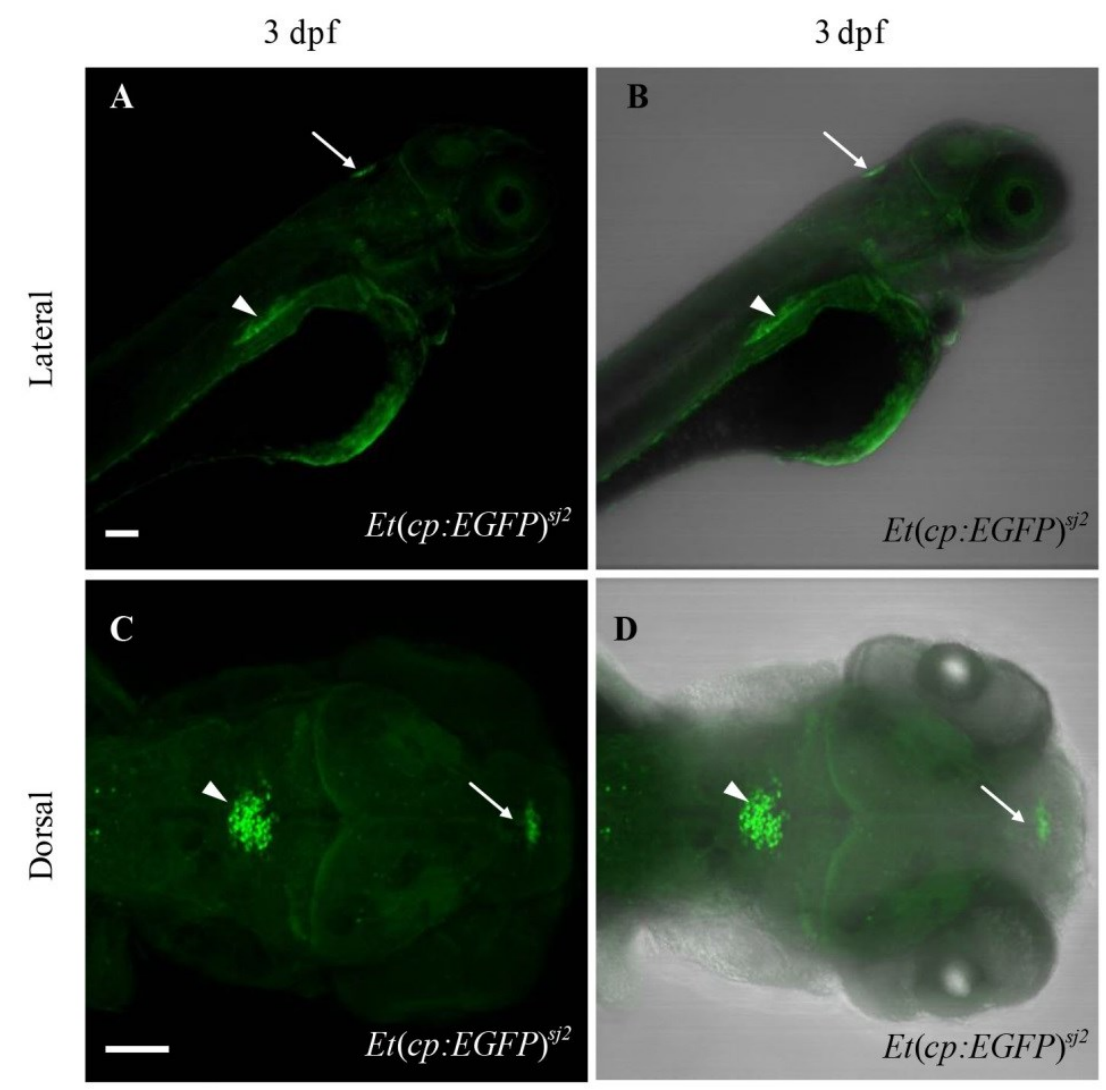

Figure 2-6. GFP Expression Is Restricted to the dCP, mCP, and Swimbladder.

Lateral confocal fluorescent image (A) and merged image (B) of Et(cp:EGFP) $)^{s j 2}$ larvae at 3 dpf. GFP expressed in the mCP (arrow), and swimbladder (arrowhead). Dorsal confocal fluorescent (C) and merged image (D) of $E t(c p: E G F P)^{s j 2}$ larvae at $3 \mathrm{dpf}$. GFP is expressed in the $\mathrm{mCP}$ (arrowhead) and dCP (arrow). dCP, diencephalic choroid plexus; $\mathrm{mCP}$, myelencephalic choroid plexus. Scale bars are $50 \mu \mathrm{m}$. 
To gain further insight into the location of the CP in relation to other CNS structures, we used whole-mount IHC to stain Et(cp:EGFP) ${ }^{s j 2}$ larvae with a Zpr-1 antibody labeling cone photoreceptors of the eye and the pineal gland. In other teleosts and mammals, the pineal gland directly interacts with the $\mathrm{CP}$ of the third ventricle (Omura et al., 1985; Skinner and Malpaux, 1999). Although the dCP is distinct from the pineal gland, we found a close association between the two structures (Figure 2-4B). Transverse sections showed the dCP positioned beneath the pineal gland (Figure 2-4C). Additional transverse sections through the mCP showed a cluster of GFP-positive epithelial cells directly beneath the skin surface (Figure 2-4D). GFP expression in the $\mathrm{dCP}$ and $\mathrm{mCP}$ was retained throughout development and at later stages, as shown in an one-month-old $E t(c p: E G F P)^{s j 2}$ (Figure 2-4E and 2-4F).

To further demonstrate that this is indeed the $\mathrm{CP}$, we cut sections from adult brains and identified GFP expression in a structure historically referred to as the saccus dorsalis (Figure 2-7). This structure is analogous to the mammalian $\mathrm{CP}$ of the third ventricle and the developmental $\mathrm{dCP}$ in zebrafish. The saccus dorsalis in rainbow trout (Salmo gairdneri), has been described as a folded monolayer of epithelium that develops out of the diencephalic roof plate (Jansen et al., 1976). Because the saccus dorsalis has also been described to partially cover the pineal stalk (Tsuneki, 1986), similar to what we show in Figure 2-4B, we conclude that this is the CP of the third ventricle in zebrafish.

\section{Zebrafish CP Expresses the Tight-Junction Claudin 5 and Zonula Occludens-1}

The mammalian CP expresses many tight-junction proteins such as claudins (Kratzer et al., 2012; Lippoldt et al., 2000), occludens (Lagaraine et al., 2011) and junction adhesion molecules (Lagaraine et al., 2011) to create a physical barrier between the blood and CSF. To demonstrate that the zebrafish CP express tight junction proteins similar to mammals, we studied Cldn5 expression due to accessibility to an antibody that cross-reacts with zebrafish Cldn5 (Xie et al., 2010; Zhang et al., 2010). As predicted, whole-mount IHC analysis revealed Cldn5 expression at the cell membrane of zebrafish CPe (Figure 2-8). Cldn5 expression was also observed in the eyes, blood vessels, and neighboring ependymal cells (Figure 2-8B and 2-8C). In addition, we analyzed ZO-1, another tight junction protein expressed in CNS barriers. Not surprisingly, whole mount IHC revealed ubiquitous ZO-1 expression (Figure 2-9A-C). However, transverse sections through the $\mathrm{mCP}$ acquired at a higher magnification demonstrated co-localization with the GFP positive cells of the CP indicating that along with Cldn5, $\mathrm{ZO}-1$ is also present at the BCSFB (Figure 2-9D).

\section{Zebrafish CP Possesses Transporter Activity}

In mice, multidrug resistance transporters, such as MRP1, are expressed on the basolateral side of CPe and regulate drug accumulation in the CSF (Wijnholds et al., 2000). In addition to drug transport, MRP1 and MDR1, efflux fluorescent dyes such as rhodamine 123 (Saengkhae et al., 2003). To the best of our knowledge, no previous 

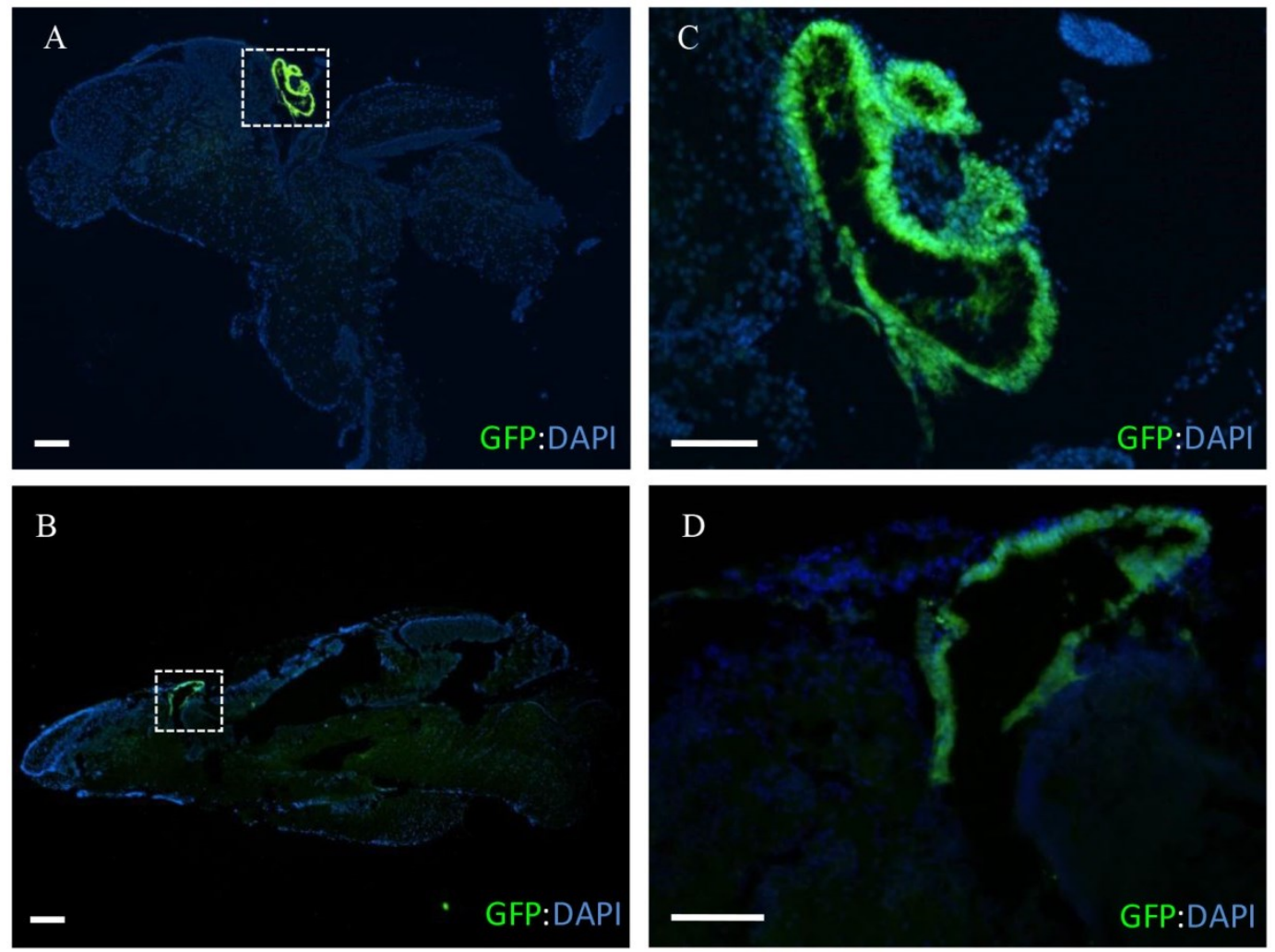

Figure 2-7. The Adult Et(cp:EGFP $)^{\text {sj2 }}$ Expresses GFP in the Saccus Dorsalis.

Sagittal sections through adult brains were labelled with rabbit anti-GFP antibody (green) and counterstained with DAPI (blue). (A-B) shows GFP expression in the adult CP of the third ventricle, also known as the saccus dorsalis in teleosts. Dashed-line boxes refer to magnified images shown in (C-D). Scale bars are $100 \mu \mathrm{m}$. 

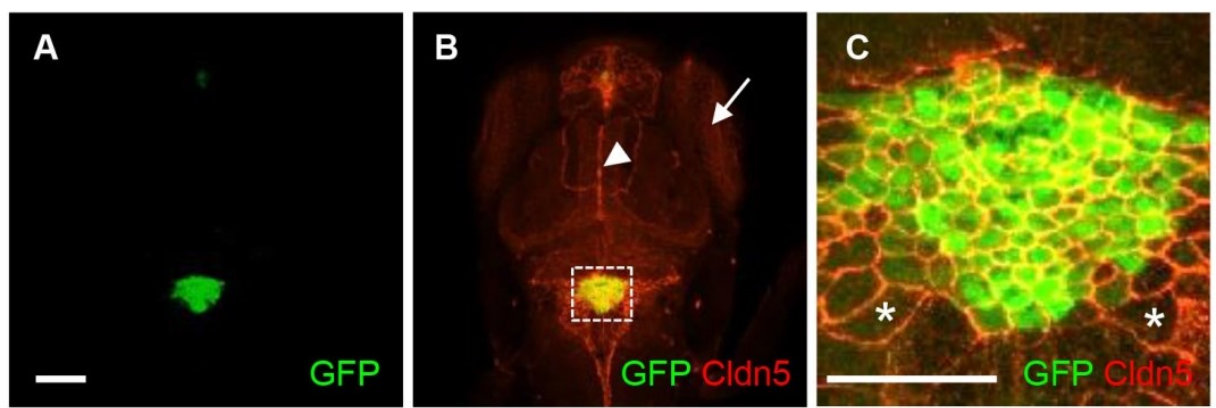

Figure 2-8. The Zebrafish CP Possesses the Tight Junction, Claudin 5.

(A) Whole-mount immunohistochemical staining of $E t(c p: E G F P)^{s j 2}$ larvae at 4 dpf with GFP labeling the dCP and mCP. (B) Whole-mount immunohistochemical staining with Claudin 5 antibody (red) showed expression in the eye (arrow), surface vessels (arrowhead), and CP. (C and C') Overlay of GFP and Claudin 5 staining revealed Claudin 5 expression surrounding the perimeter of $\mathrm{CPe}$ and ependymal cells. Examples of ependymal cells are shown with an asterisk $(*)$. Images in (A) and (B) taken at 20× magnification and (C) at $40 \times$ magnification. 

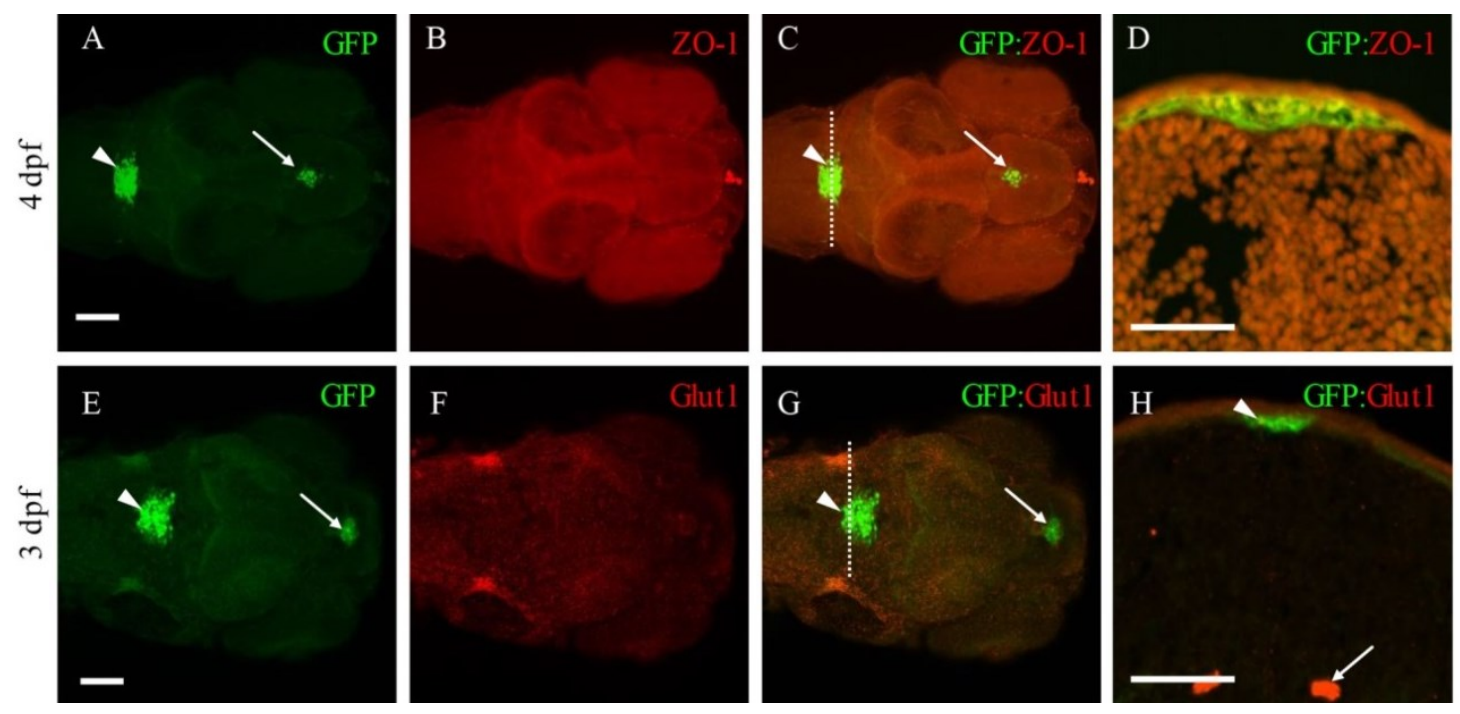

\section{Figure 2-9. Z ZO-1 and Glut1 Expression in the CP.}

(A) Dorsal confocal imaged of Et(cp:EGFP) larvae at 4 dpf with GFP expression (green) in the dCP (arrow) and mCP (arrowhead). (B) Dorsal confocal imaged of Et(cp:EGFP) larvae at 4 dpf with ubiquitous ZO-1 expression (red) throughout the head and eyes. (C) Merged image of GFP and ZO-1 expression with dCP (arrow) and mCP (arrowhead). Dotted line is representative of the section through the $\mathrm{mCP}$ in (D). (D) Transverse section through the mCP shows ZO-1 expression (red) co-localizes with GFP expression (green) in the mCP (yellow). (E) Dorsal confocal imaged of Et(cp:EGFP) larvae at $3 \mathrm{dpf}$ with GFP expression (green) in the $\mathrm{dCP}$ (arrow) and $\mathrm{mCP}$ (arrowhead). (F) Dorsal confocal imaged of Et(cp:EGFP) larvae at $3 \mathrm{dpf}$ with ubiquitous Glut1 expression (red) throughout the head and eyes. (G) Merged image of GFP and Glut 1 expression with $\mathrm{dCP}$ (arrow) and mCP (arrowhead). (H) Transverse section through the mCP shows Glut 1 expression (red) on the surface and brain vasculature (arrow) and GFP expression (green) in the $\mathrm{mCP}$ (arrowhead). Scale bars in (A) and (E) are $50 \mu \mathrm{m}$ and in (D) and (H) $25 \mu \mathrm{m}$. 
studies have demonstrated transporter function in zebrafish CP. To determine whether transporters contribute to the BCSFB at the zebrafish CP, we intravenously injected rhodamine 123 into the $E t(c p: E G F P)^{s j 2}$ line at $4 \mathrm{dpf}$ and observed its accumulation within the CPe (Figure 2-10A-C). After injecting rhodamine 123 into the brain ventricle/CSF, we also observe accumulation in CPe (Figure 2-11A-D) indicating that independent of what interface the rhodamine 123 resides (whether blood or CSF), the dye is transported by the $\mathrm{CP}$.

Another transporter that is highly expressed in the CP is OAT3 (Keep and Smith, 2011) and fluorescein is taken up by the transporter on the apical side of CPe in a $\mathrm{Na}^{+}$-dependent manner (Sykes et al., 2004). Because fluorescein is a low-molecular-weight green fluorescent dye, we soaked Casper zebrafish in $50 \mu \mathrm{M}$ fluorescein to observe accumulation in the CP. The dye was ingested in the gut, but also entered the circulation and concentrated in the dCP and $\mathrm{mCP}$ (Figure 2-10D and 2-10E). Fluorescein injected into the brain ventricle had a similar pattern of accumulation in the $\mathrm{mCP}$ and dCP (Figure 2-11E-H). Additionally, we looked at transporters such as Glut 1 . Glut1 is a well-known marker of the BBB (Cullen et al., 2011; Daneman and Rescigno, 2009; Dermietzel et al., 1992; Klepper and Voit, 2002; Zlokovic, 2008). However we did not observe its expression within the zebrafish CP (Figure 2-9E-H).

\section{Zebrafish CP Possesses Size-Selective Barrier Properties}

To date, there have been no studies on paracellular barrier properties of the zebrafish CP (Abdelilah-Seyfried, 2010). To examine the function of the zebrafish CP in regulating passage from the bloodstream into the $\mathrm{CSF}$, we intravenously injected fluorescently labeled dextrans of various molecular weights into Casper at 2, 3, and $4 \mathrm{dpf}$. Time-lapse imaging at $2 \mathrm{dpf}$, before the CP has fully developed, showed that $3-\mathrm{kDa}$ fluorescein and 10-kDa rhodamine dextrans leaked from the bloodstream into the brain ventricle, whereas little to no 40-kDa fluorescein dextran escaped from the bloodstream into the ventricle (Figure 2-12A). By $3 \mathrm{dpf}$, there was still a significant difference with a $P$ value of $<0.0001$ between the $3-\mathrm{kDa}$ and $10-\mathrm{kDa}$ dextrans and the $40-\mathrm{kDa}$ dextran; however, the overall permeability was lower at $3 \mathrm{dpf}$ compared to $2 \mathrm{dpf}$ (Figure 2-12B). By 4 dpf, when the CP was fully formed, there were no significant differences between the individual tracers (Figure 2-12B). Intravenous injection of the 10-kDa dextran into $E t(c p: E G F P)^{s j 2}$ larvae at 2 dpf showed leakage into the brain ventricle as the CP continued to develop. Once the CP formed by $4 \mathrm{dpf}$, the dextran did not accumulate within the brain ventricle (Figure 2-13A). In addition, the 3-kDa and 10-kDa dextrans were significantly more permeable at $2 \mathrm{dpf}(P<0.0001)$ compared to $3 \mathrm{dpf}$ and $4 \mathrm{dpf}$, but there was no significant difference in permeability for the 40-kDa dextran (Figure 2-13B). 

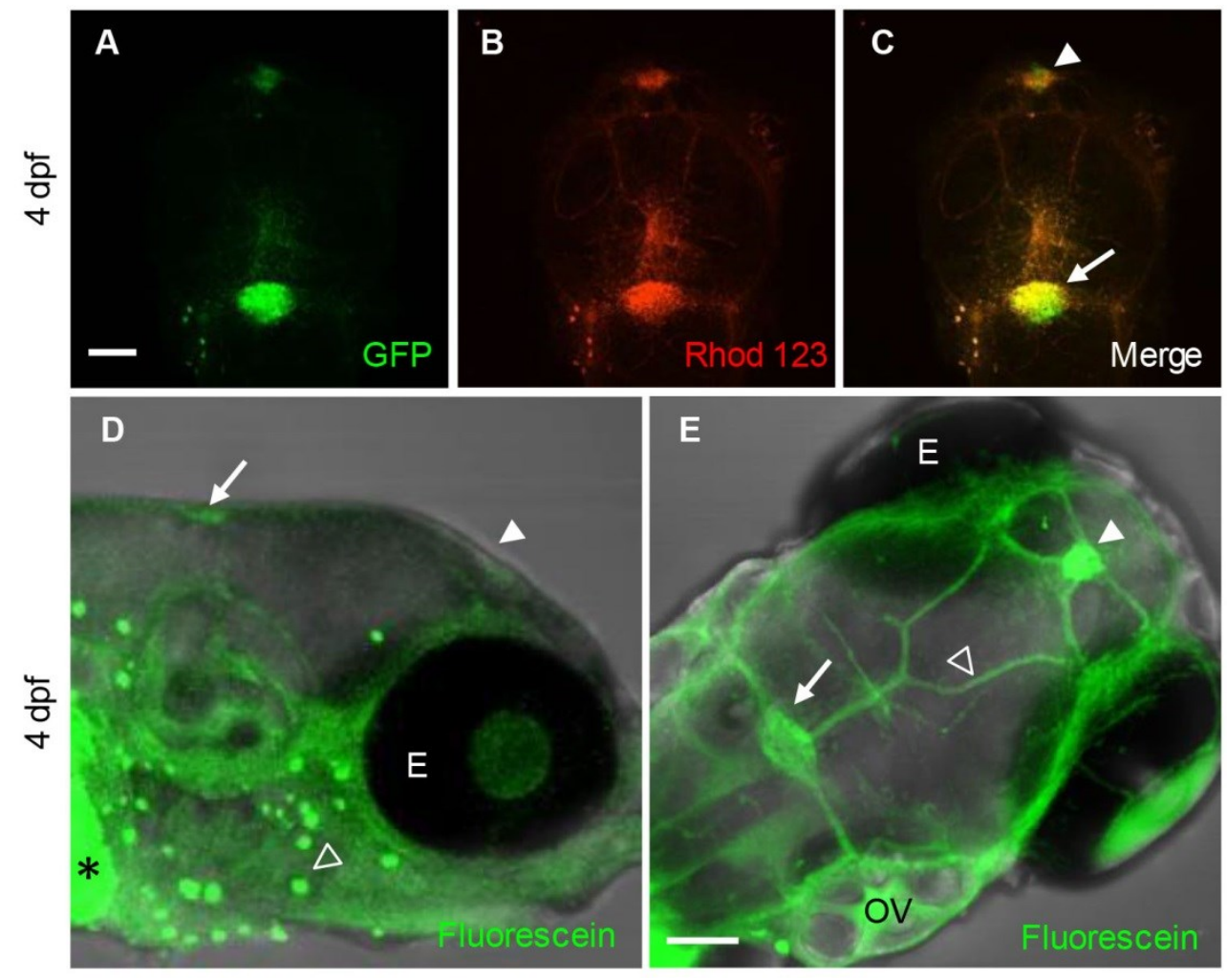

Figure 2-10. The Zebrafish CP Possesses Transporter Properties.

(A) Live imaging of $E t(c p: E G F P)^{s j 2}$ larvae at 4 dpf showed GFP expression in the CP. $(\mathrm{B}, \mathrm{C})$ Accumulation of rhodamine 123 in the $\mathrm{dCP}$ (arrowhead) and $\mathrm{mCP}$ (arrow). (D) Side view of live Casper zebrafish treated at $4 \mathrm{dpf}$ with $50 \mu \mathrm{M}$ fluorescein for $4 \mathrm{~h}$ showed accumulation of fluorescein (represented in green) in the dCP (filled arrowhead) and $\mathrm{mCP}$ (arrow) along with the gut (asterisk), and lateral line (open arrowhead). (E) Similar image as in (D) except for dorsal view showing accumulation in the $\mathrm{dCP}$ (filled arrowhead), $\mathrm{mCP}$ (arrow), and overlying vasculature (open arrowhead). Abbreviations: $\mathrm{dCP}$, diencephalic CP; mCP, myelencephalic CP; E, eye; OV; Otic vesicle. Scale bar is $50 \mu \mathrm{m}$. 

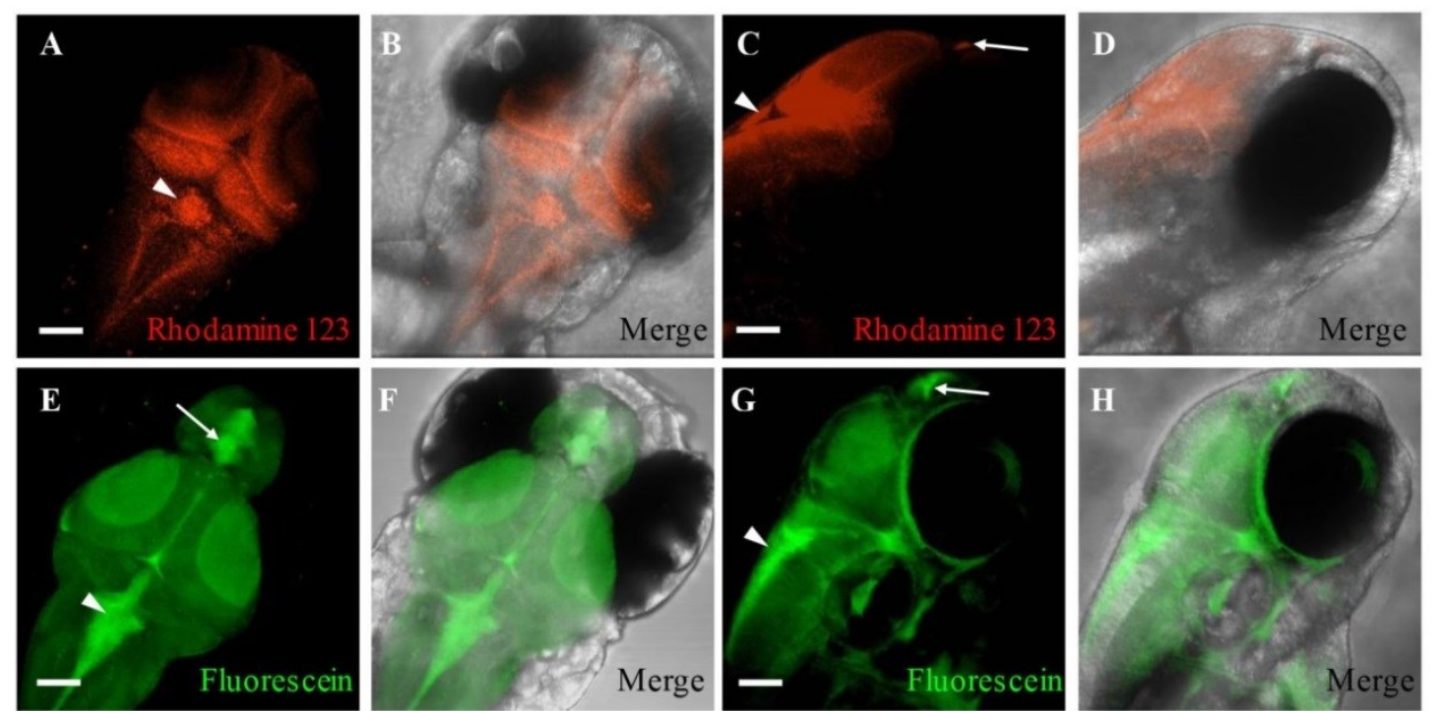

Figure 2-11. Rhodamine 123 and Fluorescein Ventricle Injection Demonstrates Transporter Activity in the CP.

Dorsal fluorescent (A) and merged (B) confocal image of 4dpf Casper larvae with Rhodamine 123 (red) injection into the brain ventricle. The rhodamine 123 concentrates within the mCP (arrowhead). Rhodamine 123 also can fluoresce green and co-localizes with red emission (not shown). Lateral fluorescent (C) and merged (D) confocal image of 4dpf Casper larvae with Rhodamine 123 (red) injection into the brain ventricle. The rhodamine 123 concentrates within the $\mathrm{mCP}$ (arrowhead) and $\mathrm{dCP}$ (arrow). Dorsal fluorescent (E) and merged (F) confocal image of 4dpf Casper larvae with fluorescein (green) injected into the brain ventricle. Fluorescein concentrates within the $\mathrm{mCP}$ (arrowhead) and dCP (arrow). Lateral fluorescent (G) and merged (H) confocal image of $4 \mathrm{dpf}$ Casper larvae with fluorescein (green) injected into the brain ventricle. Fluorescein concentrates within the $\mathrm{mCP}$ (arrowhead) and $\mathrm{dCP}$ (arrow). Abbreviations: $\mathrm{mCP}$, myelencephalic choroid plexus; dCP, diencephalic choroid plexus. Scale bar is $50 \mu \mathrm{m}$. 

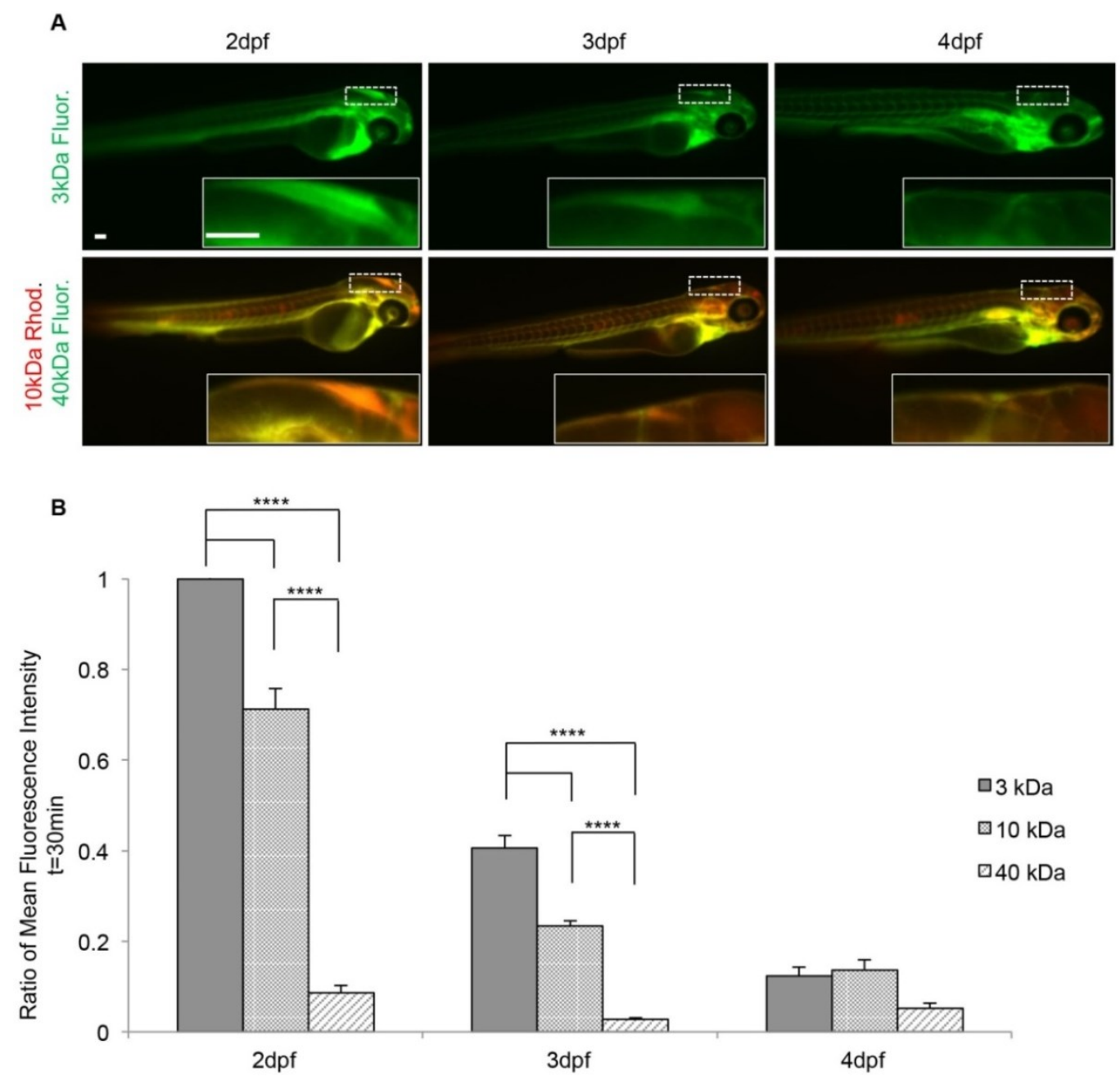

Figure 2-12. The Zebrafish CP Is Functional and Possesses Size-Selective Properties.

Casper zebrafish were intravenously injected with fluorescent tracers at 2, 3, and $4 \mathrm{dpf}$ to identify tracer leakage from blood into the CSF. (A) The top panel shows severe leakage of the 3-kDa fluorescein dextran into the brain ventricle (inset) at $2 \mathrm{dpf}$, minor leakage at $3 \mathrm{dpf}$, and little, if any, dextran penetration at $4 \mathrm{dpf}$. The bottom panel shows permeability of the $10-\mathrm{kDa}$ rhodamine dextran at $2 \mathrm{dpf}$ (inset), which decreases by $4 \mathrm{dpf}$. The 40-kDa fluorescein dextran was the least permeable at each developmental time point. Images were taken at $1 \mathrm{~h}$ post-injection. (B) A one-way ANOVA shows that the $3-\mathrm{kDa}$ fluorescein dextran and the $10-\mathrm{kDa}$ rhodamine dextran are significantly more permeable (i.e., greater mean fluorescence intensity) than the 40-kDa fluorescein dextran at $2 \mathrm{dpf}$ and $3 \mathrm{dpf}$. By $4 \mathrm{dpf}$, there was no significant difference in fluorescence intensity. Measurements are expressed as mean $\pm \mathrm{SE}$ (standard error) for $n=7 ; * * * p<0.0001$. Abbreviations: Rhod, rhodamine dextran; Flour, fluorescein dextran. Scale bar is $50 \mu \mathrm{m}$. 

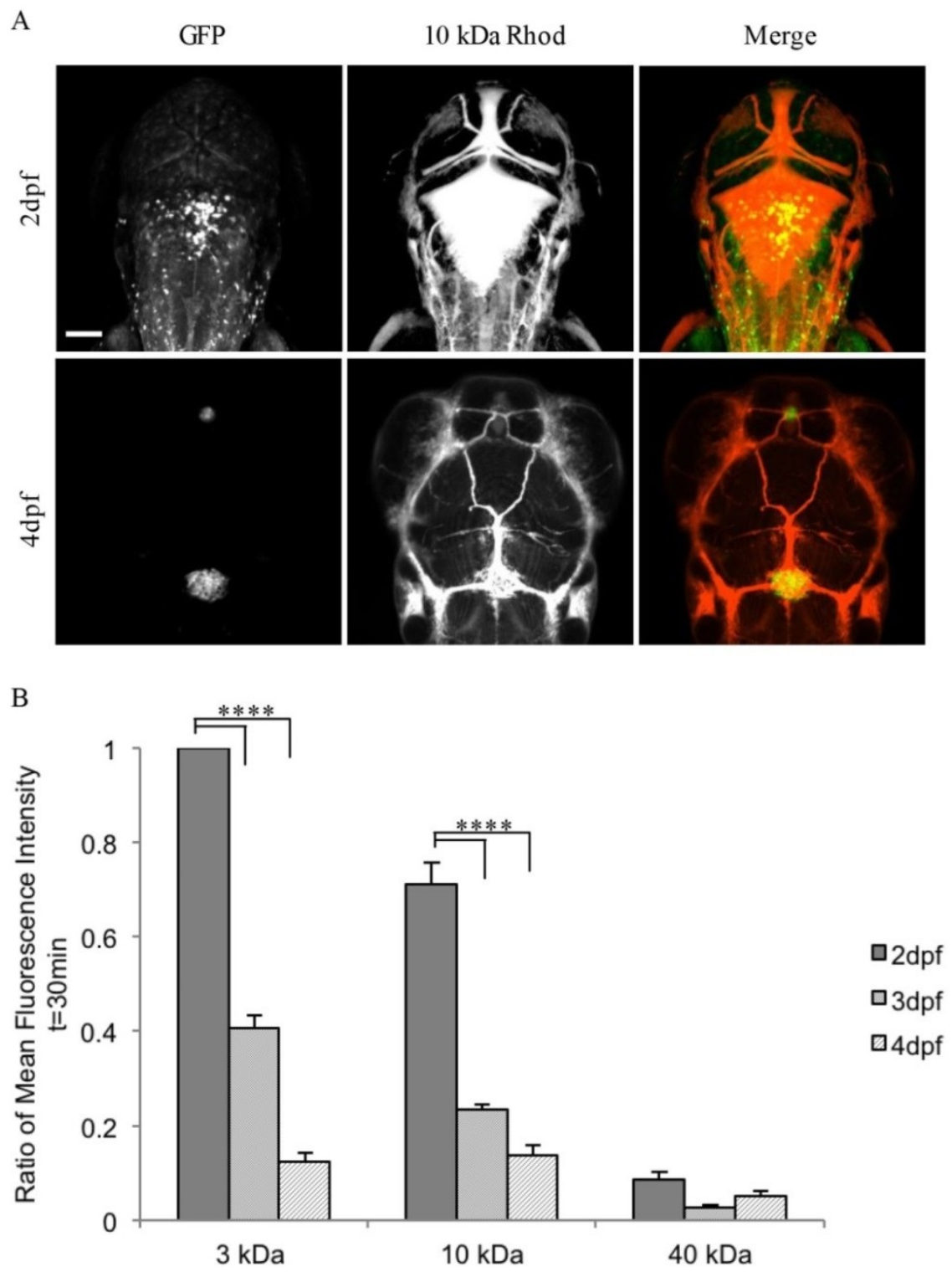

Figure 2-13. The Zebrafish CP Becomes Size-Selective as It Develops.

(A) $E t(c p: E G F P)^{s j 2}$ larvae were intravenously injected with a $10 \mathrm{kDa}$ rhodamine-dextran and the $\mathrm{CP}$ was imaged dorsally by confocal microscopy. At $2 \mathrm{dpf}$, the $\mathrm{CP}$ is not fully developed as observed by GFP expression and the $10 \mathrm{kDa}$ rhodamine-dextran inundates the brain ventricle. By $4 \mathrm{dpf}$, the $\mathrm{CP}$ is fully formed and the $10 \mathrm{kDa}$ rhodamine-dextran is retained within the vasculature and does not enter the brain ventricle. (B) A One-Way ANOVA analysis revealed that the $3 \mathrm{kDa}$ fluorescein dextran and $10 \mathrm{kDa}$ rhodamine dextran have a significantly higher fluorescent intensity in the brain ventricle (i.e. more permeable) at $2 \mathrm{dpf}$ compared to 3 and $4 \mathrm{dpf}$. There is no significant difference between developmental time points for the $40 \mathrm{kDa}$ fluorescein dextran. Measurements are means $\pm \mathrm{SE}$ for $n=7$; ****p $<0.0001$. Scale bar is $50 \mu \mathrm{m}$. 


\section{Discussion}

The goal of our study was to establish zebrafish as a functionally relevant model to study CP development. Although the CP has been studied in various in vivo and in vitro models (Dohrmann, 1970; Strazielle et al., 2003), the developmental and genetic aspects of this structure remain poorly understood largely due to the lack of innovative strategies and functionally relevant systems (Saunders et al., 2008). Also, other vertebrate models do not easily allow for $\mathrm{CP}$ development to be visualized in real time. To overcome this limitation, we created an enhancer trap zebrafish line, Et(cp:EGFP $)^{s j 2}$ expressing EGFP within the $\mathrm{dCP}$ and $\mathrm{mCP}$. Using this line, $\mathrm{CP}$ development was easily observed in a live vertebrate by monitoring EGFP expression.

Previous studies have also generated enhancer trap lines in zebrafish expressing GFP in the CP (Bill et al., 2008; Garcia-Lecea et al., 2008). However, there are variations within these lines, including the subsets of cells expressing GFP. For example, in the $E t^{M n 16}$ line (Bill et al., 2008), only a subset of cells of the mCP express GFP. The SqET33-E20 (Gateways) line (Garcia-Lecea et al., 2008) has additional GFP expression in the rhombomeres. In our study, there was specific GFP expression in the $\mathrm{dCP}, \mathrm{mCP}$, and swimbladder. In regards to GFP expression in the swimbladder, studies have shown that Shh is expressed in the epithelial cells lining the swimbladder (Winata et al., 2009). Interestingly, the $\mathrm{CP}$ of other vertebrates has also been compared to the mammalian lung in a study by Nielsen and Dymecki (2010) demonstrating that Shh is required for the synchronized outgrowth of the CP and fenestrated vasculature, similar to the need for Shh in the coordinated outgrowth of the lung (Nielsen and Dymecki, 2010). Since the zebrafish swimbladder has been compared to the mammalian lung (Winata et al., 2009), this suggests that there are common underlying developmental pathways, such as Shh, required in both the $\mathrm{CP}$ and swimbladder and the GFP expression in this enhancer trap line may be regulated by one of these developmental cues.

By performing confocal time lapse imaging on the $E t(c p: E G F P)^{s j 2}$ line, we observed a few GFP positive spots in the head and eyes. We attributed these green spots to the high gain we used to acquire the time-lapse. Because we start the imaging before the CP has formed, we increased the gain normally used to acquire snapshot images at 3 dpf in order to insure that we had sufficient exposure to capture GFP positive cells as they develop into the CP. The variations in other enhancer trap lines may be due to transgenic DNA constructs integrating into different regions of the genome. As seen with the other zebrafish CP enhancer trap lines, we could not identify the enhancer element regulating GFP expression, likely because of the enhancer's ability to act at remote distances upstream or downstream of the target gene.

We have demonstrated BCSFB properties in zebrafish by identifying tight junction proteins and transporter activity. We have shown Cldn5 and ZO-1 expression within the CP. Identifying these tight junctions confirms what is known in the literature where ZO-1 interacts with Claudins to anchor them within the cell membrane (Brown and Davis, 2002; Stamatovic et al., 2008). Using our Et(cp:EGFP $)^{s j 2}$ line, we demonstrated that the zebrafish $\mathrm{CP}$ possess a chemical barrier that transports and 
concentrates rhodamine 123 and fluorescein in the CPe. Rhodamine 123 directly interacts with MRP1 and MDR1 in an ATP-dependent manner (Daoud et al., 2000; Shapiro and Ling, 1998). Because previous studies have shown the specificity of rhodamine 123 to MDRs, similar drug transporters are likely expressed in the zebrafish CP. Studies in mice have identified known transporters of rhodamine 123 such as MRP1 to be localized at the basolateral side of CP epithelia and keep drugs out of the CSF (Keep and Smith, 2011; Wijnholds et al., 2000). Our findings also confirm that transporters are present in the zebrafish CP by showing that: when coming from the bloodstream, rhodamine 123 accumulates within the CP and is prevented from entering the CSF. Likewise, when injected directly into the CSF, the CP functions in removing rhodamine 123 from the fluid as is also shown by dye accumulation within CP epithelia.

OAT3, also known as solute carrier family 22 group 8 (Slc22a8) is expressed in the kidney, intestine, retinal pigment, brain, and CP (Sweet et al., 2003). Its function has been commonly studied by treating isolated mouse CPe cells with fluorescein and incubating them in artificial CSF. A loss of OAT3 results in reduced fluorescein accumulation (Sweet et al., 2003). A model system using intact CP from the dogfish shark demonstrated $\mathrm{Na}+$-dependent accumulation of fluorescein in the epithelia via OATs (Villalobos et al., 2002). Our real-time studies also showed in vivo fluorescein accumulation within the $\mathrm{CPe}$, which was indicative of transport activity. Although the current zebrafish genome database contains two annotated Oat proteins zfOat (NP_996960) and zfOatX (NP_004781) (Aslamkhan et al., 2006), there are no expression data for these genes. Future studies will investigate whether specific Oats are expressed in the zebrafish CP and contribute to fluorescein transport. Additionally, while we did not observe Glut 1 expression within the zebrafish CP, a recent review by Keep and Smith mention that the $\mathrm{BBB}$, not the $\mathrm{BCSFB}$ is the major source of glucose transport into the brain due to its close proximity to the brain itself and that the $\mathrm{CP}$ has only a minor role in this process (Keep and Smith, 2011).

We demonstrated barrier function in the zebrafish CP by intravenously injecting fluorescent tracers of different molecular weights and observing permeability across the $\mathrm{CPe}$ into the CSF. Once the CP fully formed by $4 \mathrm{dpf}$, the smallest tracer tested, a 3-kDa fluorescein dextran, was no longer permeable across the BCSFB as it was in earlier developmental stages. This result indicated a tightening of the barrier as it develops. There appears to be a correlation between CP formation and decreased permeability, suggesting that a physical barrier is preventing molecule entry rather than being cleared by a CSF drainage pathway. For example, this is demonstrated when we injected the $40-\mathrm{kDa}$ tracer into the bloodstream on day 4 and never observed tracer accumulation within the ventricle throughout the course of the time-lapse. Rather than suggesting that the tracer is being cleared from the ventricle by CSF flow, we observed that the tracer never entered the ventricle in the first place. This indicated to us that a physical barrier is preventing the dextran from crossing the barrier. Had we observed accumulation of the dextran within the CSF/brain-ventricle after injection, and then observed a decrease in the amount of tracer in the ventricle over time, then we would suggest that CSF flow is the contributing factor. In immature brain barriers, it has been reported that the accumulation of molecules within the CSF is not due to increased permeability of the barrier but 
reduced CSF flow (Saunders et al., 2008). In contrast, more mature brain barriers have increased CSF flow that limits the accumulation of molecules within the ventricular space (Saunders et al., 2008). Our study showed that after CP formation, there was no tracer penetration into the ventricle indicating that a decrease in tracer accumulation in the brain ventricle was not attributable to an increase in CSF clearance, but barrier properties of the $\mathrm{CP}$. The decrease in barrier permeability as the $\mathrm{CP}$ develops establishes the zebrafish $\mathrm{CP}$ as a functioning organ with size-selective barrier properties.

\section{Conclusion}

In summary, the goal for this investigation was to establish zebrafish as vertebrate model to study the $\mathrm{CP}$. By characterizing the $\mathrm{CP}$ in zebrafish, we have demonstrated that it not only possesses barrier properties similar to mammals, such as have tight junctions and transporter activity, but also functions in regulating entry into the CSF from the bloodstream and transporting molecules out of the CSF. These findings will pave the way for future investigations using zebrafish as a model for $\mathrm{CP}$ development and function while providing tools for investigating barrier properties in mutants with $\mathrm{CP}$ abnormalities. 


\section{CHAPTER 3. FORWARD GENETIC SCREEN IDENTIFIES CHOROID PLEXUS MUTANTS}

\section{Introduction}

Although the $\mathrm{CP}$ of the lateral brain ventricles was documented several centuries ago by Herophilus, (Dohrmann, 1970; Dziegielewska et al., 2001), there has been little research aimed towards genetically dissecting the pathways essential for CP formation and function. To identify zebrafish with gene mutations specific to CP development, we performed a small-scale ENU-mutagenesis screen using Et(cp:EGFP) $)^{s j 2}$ and identified 10 recessive mutant lines. These mutants were classified into five categories on the basis of EGFP expression and $\mathrm{mCP}$ morphology. We hypothesize that this unbiased approach will uncover novel genes and pathways essential for CP development. The cloning and characterization of these mutants will provide important insights into the genetic mechanisms regulating $\mathrm{CP}$ formation and function in health and disease.

Forward genetic screens have been performed in various multicellular organisms such as plants (Meinke and Sussex, 1979), flies (Nusslein-Volhard and Wieschaus, 1980), worms (Sin et al., 2014), zebrafish (Driever et al., 1996), and mice (Hrabe de Angelis et al., 2000; Nolan et al., 2000) and have been very successful in discovering genes involved in embryonic patterning, organ development, and behavior (Horvitz, 1999; Muto et al., 2005; Stemple and Driever, 1996). The genetic plant model Arabidopsis thaliana, is a useful model system due to its small genome and minor amount of repetitive sequences (Page and Grossniklaus, 2002). Mutants in A. thaliana have been screened for phenotypes such as leaf or flower morphology, and in doing so; a number of genes have been identified such as those involved in biosynthetic pathways (Yang et al., 2011). Thomas Hunt Morgan was the first to use the fruit fly Drosophila melanogaster; a genetic model system having only four chromosomes and easily identifiable phenotypes such as eye color or wing vein patterning (St Johnston, 2002). Utilizing this model for forward genetic screening identified genes such as Hedgehog, which was found to play a role in segment polarity (Nusslein-Volhard and Wieschaus, 1980). These findings later resulted in Christiane Nüsslein-Volhard and Eric Wieschaus being awarded a Nobel Prize for their work (St Johnston, 2002).

Another classical genetic invertebrate model is the nematode Caenorhabditis elegans. This simple organism is excellent for studying nervous system development having only 302 neurons that are easily tracked using fluorescent reporter lines (Jorgensen and Mango, 2002). In addition to a nervous system, they also have muscles, epidermis, reproductive structures, and a gastrointestinal tract (Jorgensen and Mango, 2002). Sydney Brenner was the first to suggest using C. elegans as a genetic model system and he along with John Sulston and Robert Horvitz went on to receive a Nobel Prize for their work (Moller, 2002) identifying genes involved in the cell death pathway such as ced-3 (Ellis and Horvitz, 1986), correspond to Caspase-1 in mammals (Thornberry, 1997). Forward genetic screens, including large-scale ENU mutagenesis screens can also be performed in vertebrate models such as mice (Kile and Hilton, 2005). 
Genome-wide analyses have identified important genes such as Min (multiple intestinal neoplasia), also known as Adenomatous Polyposis Coli (APC), where young mice who inherit a dominant mutation in this gene are predisposed to developing adenomas in the intestine (Moser et al., 1990; Su et al., 1992). Mouse embryos can be difficult to screen in utero, however embryonic lethal mutations have been identified (Garcia-Garcia et al., 2005). While mice provide an advantage by greatly mimicking human development, large numbers of offspring for screening and the ability to visualize internal organs in real time is limited in this system.

Zebrafish have long been known for their usefulness in forward genetic screening as described in Zebrafish Genetic Screening Strategies on pg. 17. While many of these early studies focused on embryonic patterning or organ development, genetic screens in zebrafish can also be used to identify behavioral patterns. In 2005, a genetic screen was published that identified mutants with visual behavior defects using screening tools such as optokinetic response to monitor eye movement patterns and identify vision abnormalities (Muto et al., 2005). In regards to CNS development, genetic screens have also been used in isolating zebrafish with defects in brain ventricle development (Lowery et al., 2009). By injecting fluorescent dyes into the brain ventricles, they were able to categorize four classes of brain ventricle mutants based simply on morphological development. Interestingly, related genes identified in these mutants also grouped together in these four classes indicating that screening based on morphology is a good indicator for identifying classes of genes later on in genetic mapping (Lowery et al., 2009). While a few genes and signaling pathways have been identified to be essential in $\mathrm{CP}$ formation as mentioned in Signaling Pathways and the Choroid Plexus on pg. 7, the CP would greatly benefit from an unbiased approach to discover genes essential for its development and function. Because the $\mathrm{CP}$ is visible and accessible in live transgenic zebrafish models, mutants can be easily identified by screening for GFP expression and patterning. As we demonstrate in the following study, zebrafish can be used to discover phenotypes in a particular cell type or tissue by screening for fluorescence intensity and patterning throughout development.

\section{Materials and Methods}

\section{ENU Mutagenesis and Forward Genetic Screening}

Twenty Et(cp:EGFP) $)^{s j 2}$ males were treated three times with $3 \mathrm{mM}$ ENU (Sigma) for $1 \mathrm{~h}$ each at weekly intervals as described previously (de Bruijn et al., 2009; Pelegri, 2002). After one month, ENU-treated males were mated to Et(cp:EGFP) $)^{s j 2}$ females to produce the $F_{1}$ generation. $F_{1}$ pairwise crosses were performed to produce $F_{2}$ families. $\mathrm{F}_{2}$ families that did not contain at least four male/female pairs were sacrificed. Pairwise crosses for each $\mathrm{F}_{2}$ family were done at least six times if possible to identify $\mathrm{F} 3$ offspring with homozygous recessive mutations. $F_{2}$ pairs deemed heterozygous for a recessive mutation were maintained in miniboxes for use in genetic mapping experiments. To screen for $\mathrm{CP}$ mutants, $\mathrm{F}_{3}$ larvae were anesthetized at $4 \mathrm{dpf}$ and visualized using a Nikon 
SMZ1500 epifluorescence stereomicroscope to identify abnormal EGFP expression or patterning in the CP. A mutant line was confirmed if approximately $25 \%$ of the total offspring displayed a CP phenotype.

\section{Live Confocal Imaging and Time-Lapse Movies}

Scans of DIC and GFP images were acquired on a Nikon C1Si confocal microscope using a 10x and 20x objectives at $4 \mu \mathrm{m}$ intervals to create a $\mathrm{z}$-stack maximum intensity projection image. To compare CP development, wild-type and mutant larvae were imaged on a Nikon C1Si confocal microscope beginning at $54 \mathrm{hpf}$ with images acquired every 30 min over a $48 \mathrm{~h}$ period. Supplemental movies run for the $48 \mathrm{~h}$ period except for SupplementalMovie6, which runs for $42 \mathrm{~h}$ (SupplementalMovies1-7). All images were analyzed using NIS-Elements AR 4.0 software.

\section{Results}

\section{Forward Genetic Screen Identifies CP Mutants}

We initiated a small-scale forward genetic screen to identify mutants with $\mathrm{CP}$ abnormalities. This screen focused primarily on mCP defects. Table 3-1 shows the numbers of fish generated from this screen. Briefly, twenty $\mathrm{F}_{0} E t(c p: E G F P)^{s j 2}$ males were mutagenized with ENU. One month after treatment, ENU efficiency was tested by mating the males to Casper and screening for nacre or roy phenotypes (lacking melanocytes and iridiphores, respectively). Approximately 1 in $250(0.4 \%)$ larvae showed the nacre or roy phenotype. The mutagenized males were mated with $E t(c p: E G F P)^{s j 2}$ females to generate $409 \mathrm{~F}_{1}$ fish. Adult $\mathrm{F}_{1}$ pairs were mated together or with non-mutagenized $E t(c p: E G F P)^{s j 2}$ fish to produce $224 \mathrm{~F}_{2}$ families. Of the $\mathrm{F}_{2}$ families, 73 contained sufficient numbers to screen 4-6 pairs for mutants in the $\mathrm{F}_{3}$ generation. We set up $1330 \mathrm{~F}_{2} \times \mathrm{F}_{2}$ crosses (including re-crosses), with 421 crosses producing $\mathrm{F}_{3}$ embryos. Larvae were screened at $4 \mathrm{dpf}$ to identify CP defects based on GFP expression patterns. In total, we generated $16,339 \mathrm{~F}_{3}$ embryos and screened 12,196 .

We identified 24 mutant lines with 10 recessive mutant lines having CP deformities. In addition to CP phenotypes, each of the 10 recessive lines had other defects such as heart edema, small eyes, and small head. While several of the mutants exhibited brain necrosis, many did not develop necrosis until after the $\mathrm{CP}$ already started to form. A description of the onset of brain necrosis for each line is listed in Table 3-2. We found spontaneous mutants that had normal morphology overall, but severely abnormal or no CP (Figure 3-1). Spontaneous mutants can occur due to random errors in DNA processing such as DNA replication that result in arbitrary mutations being introduced into the genome (Moore et al., 2006). In addition, we found many mutants (some recessive and some spontaneous) with severe brain necrosis, but normal CP development. These mutants are shown in Figure 3-2. The $10 \mathrm{CP}$ mutants we describe in the following 
Table 3-1. Number of Zebrafish Generated from the Forward Genetic Screen.

\begin{tabular}{lc}
\hline Generation & Fish $(\boldsymbol{n})$ \\
\hline $\mathrm{G}_{0}$ males mutagenized & 20 \\
$\mathrm{G}_{0}$ males survived & 9 \\
$\mathrm{~F}_{1}$ & 409 \\
$\mathrm{~F}_{2}$ families started & 224 \\
$\mathrm{~F}_{2}$ families screened & 73 \\
$\mathrm{~F}_{2} \times \mathrm{F}_{2}$ crosses & 1330 \\
$\mathrm{~F}_{2}$ families that spawned & 421 \\
$\mathrm{~F}_{3}$ embryos screened & 12,196 \\
Mutant lines identified & 24 \\
Mutant lines maintained & 10 \\
\hline
\end{tabular}


Table 3-2. Characterization of Et(cp:EGFP $)^{\text {sj2 }}$ Mutants Generated from the Forward Genetic Screen.

\begin{tabular}{|c|c|c|c|c|c|c|c|}
\hline Class & Alleles & $\begin{array}{c}\text { Map } \\
\text { Position }\end{array}$ & dpf & Other Phenotypes (at 4 dpf) & $\begin{array}{c}\text { Fluorescein } \\
\text { Transport }\end{array}$ & $\begin{array}{c}\text { Onset of } \\
\text { Brain } \\
\text { Necrosis }\end{array}$ & $\begin{array}{c}\text { Initial } \\
\text { Circulation } \\
\text { Loss }\end{array}$ \\
\hline \multirow[t]{2}{*}{$\begin{array}{l}\text { I: reduced GFP } \\
\text { expression, } \\
\text { dispersed epithelia, } \\
\text { and irregularly } \\
\text { shaped CP }\end{array}$} & cp26.3 & ND & $2-3$ & $\begin{array}{l}\text { No swimbladder; brain and tail } \\
\text { necrosis; brain ventricle } \\
\text { hemorrhaging; faint heartbeat; no } \\
\text { circulation; no midbrain-hindbrain } \\
\text { ventricle boundaries }\end{array}$ & $\begin{array}{l}\text { Accumulates } \\
\text { within third } \\
\text { ventricle }\end{array}$ & $3 \mathrm{dpf}$ & $3 \mathrm{dpf}$ \\
\hline & cpl40.2 & ND & 2 & $\begin{array}{l}\text { Small head; small eyes; no } \\
\text { swimbladder; heart edema; brain } \\
\text { necrosis; faint heartbeat and } \\
\text { circulation; slightly larger forebrain } \\
\text { and wider midbrain ventricle }\end{array}$ & $\begin{array}{l}\text { Increased } \\
\text { uptake; } \\
\text { accumulates } \\
\text { within brain } \\
\text { ventricle }\end{array}$ & $4 \mathrm{dpf}$ & $4 \mathrm{dpf}$ \\
\hline \multirow[t]{2}{*}{$\begin{array}{l}\text { II: normal GFP } \\
\text { expression, smaller } \\
\text { epithelia aggregates, } \\
\text { and irregularly } \\
\text { shaped CP }\end{array}$} & $c p 9.6$ & LG 4 & 2 & $\begin{array}{l}\text { Small head; small eyes; no } \\
\text { swimbladder; heart edema; } \\
\text { occasional hydrocephalus; brain } \\
\text { necrosis; no circulation; no distinct } \\
\text { forebrain or midbrain and severely } \\
\text { reduced hindbrain }\end{array}$ & $\begin{array}{l}\text { Increased } \\
\text { uptake; } \\
\text { accumulates } \\
\text { within brain } \\
\text { ventricle }\end{array}$ & $2 \mathrm{dpf}$ & $4 \mathrm{dpf}$ \\
\hline & cp79.6 & ND & 2 & $\begin{array}{l}\text { Small head; small eyes; no } \\
\text { swimbladder; heart edema; many } \\
\text { still in chorion; brain necrosis; } \\
\text { hemorrhaging around heart; slow } \\
\text { heartbeat; little to no circulation; } \\
\text { wider midbrain ventricle }\end{array}$ & $\begin{array}{l}\text { Dispersed } \\
\text { punctate } \\
\text { staining on } \\
\text { the dorsal } \\
\text { ventricle } \\
\text { surface }\end{array}$ & $4 \mathrm{dpf}$ & $3 \mathrm{dpf}$ \\
\hline
\end{tabular}


Table 3-2. (Continued).

\begin{tabular}{|c|c|c|c|c|c|c|c|}
\hline Class & Alleles & $\begin{array}{c}\text { Map } \\
\text { Position }\end{array}$ & dpf & Other Phenotypes (at 4 dpf) & $\begin{array}{c}\text { Fluorescein } \\
\text { Transport }\end{array}$ & $\begin{array}{l}\text { Onset of } \\
\text { Brain } \\
\text { Necrosis } \\
\end{array}$ & $\begin{array}{c}\text { Initial } \\
\text { Circulation } \\
\text { Loss } \\
\end{array}$ \\
\hline $\begin{array}{l}\text { III: normal } \\
\text { GFP } \\
\text { expression, } \\
\text { smaller } \\
\text { epithelia } \\
\text { aggregates, and }\end{array}$ & $c p 79.8$ & ND & 2 & $\begin{array}{l}\text { Small head; and eyes; no } \\
\text { swimbladder; brain necrosis; } \\
\text { hemorrhaging around heart; } \\
\text { decreased heartbeat and circulation; } \\
\text { wider midbrain ventricle }\end{array}$ & $\begin{array}{l}\text { Increased } \\
\text { uptake; } \\
\text { accumulates } \\
\text { within brain } \\
\text { ventricle }\end{array}$ & $4 \mathrm{dpf}$ & $3 \mathrm{dpf}$ \\
\hline expanded CP & cp151.2 & ND & $2-3$ & $\begin{array}{l}\text { Small head; small eyes; no } \\
\text { swimbladder; brain necrosis; minor } \\
\text { swelling around head; occasional } \\
\text { hemorrhaging around heart; smaller } \\
\text { forebrain and no defined midbrain }\end{array}$ & $\begin{array}{l}\text { Increased } \\
\text { uptake; } \\
\text { accumulates } \\
\text { within brain } \\
\text { ventricle }\end{array}$ & $4 \mathrm{dpf}$ & $4 \mathrm{dpf}$ \\
\hline \multirow[t]{2}{*}{$\begin{array}{l}\text { IV: variable } \\
\text { GFP } \\
\text { expression, } \\
\text { smaller } \\
\text { epithelia } \\
\text { aggregates, and } \\
\text { expanded CP }\end{array}$} & $c p 27.5$ & LG 21 & 2 & $\begin{array}{l}\text { Small head; small eyes; no } \\
\text { swimbladder; brain necrosis; heart } \\
\text { edema; small arched body; curved } \\
\text { tail; hydrocephalus; occasional } \\
\text { hemorrhaging in brain ventricle; } \\
\text { heartbeat, but little to no circulation; } \\
\text { no defined ventricle boundaries }\end{array}$ & $\begin{array}{l}\text { Increased } \\
\text { uptake; } \\
\text { accumulates } \\
\text { within brain } \\
\text { ventricle }\end{array}$ & $\begin{array}{l}2 \mathrm{dpf} \\
\text { (localized } \\
\text { to } \\
\text { forebrain) }\end{array}$ & $3 \mathrm{dpf}$ \\
\hline & cp44.10 & ND & 2 & $\begin{array}{l}\text { Small head; small eyes; no } \\
\text { swimbladder; brain necrosis; heart } \\
\text { edema; small arched body; curved } \\
\text { tail; hydrocephalus; occasional }\end{array}$ & $\begin{array}{l}\text { Increased } \\
\text { uptake; } \\
\text { accumulates } \\
\text { within brain }\end{array}$ & $\begin{array}{l}2 \mathrm{dpf} \\
\text { (localized } \\
\text { to } \\
\text { forebrain) }\end{array}$ & $4 \mathrm{dpf}$ \\
\hline
\end{tabular}


Table 3-2. (Continued).

\begin{tabular}{|c|c|c|c|c|c|c|c|}
\hline Class & Alleles & $\begin{array}{c}\text { Map } \\
\text { Position }\end{array}$ & dpf & Other Phenotypes (at 4 dpf) & $\begin{array}{c}\text { Fluorescein } \\
\text { Transport }\end{array}$ & $\begin{array}{l}\text { Onset of } \\
\text { Brain } \\
\text { Necrosis } \\
\end{array}$ & $\begin{array}{c}\text { Initial } \\
\text { Circulation } \\
\text { Loss } \\
\end{array}$ \\
\hline \multirow{3}{*}{$\begin{array}{l}\text { V: normal GFP } \\
\text { expression, } \\
\text { compact } \\
\text { epithelia, and } \\
\text { enlarged } \mathrm{CP}\end{array}$} & & & & $\begin{array}{l}\text { hemorrhaging around heart; } \\
\text { heartbeat, but little to no circulation; } \\
\text { no forebrain or midbrain and } \\
\text { irregularly shaped hindbrain }\end{array}$ & ventricles & & \\
\hline & $c p 26.6^{b}$ & ND & 4 & $\begin{array}{l}\text { Rounded head, protruding lower jaw; } \\
\text { no swimbladder; edema around } \\
\text { heart, eyes, and gut; faint heartbeat } \\
\text { and circulation; normal brain } \\
\text { ventricle }\end{array}$ & N/A & $\mathrm{N} / \mathrm{A}$ & $4 \mathrm{dpf}$ \\
\hline & $\operatorname{cp} 105.2^{b}$ & ND & $4-5$ & $\begin{array}{l}\text { Rounded head, protruding lower jaw; } \\
\text { no swimbladder; severe edema } \\
\text { around heart, eyes, and gut; faint } \\
\text { heartbeat and circulation; slightly } \\
\text { smaller hindbrain ventricle }\end{array}$ & N/A & N/A & $6 \mathrm{dpf}$ \\
\hline
\end{tabular}

${ }^{a}$ observations recorded at $3 \mathrm{dpf} ;{ }^{b}$ observations recorded at $5 \mathrm{dpf} . \mathrm{ND}$, not determined; N/A, not applicable. Onset of Brain Necrosis and Initial Circulation Loss are observed up to $4 \mathrm{dpf}$. 

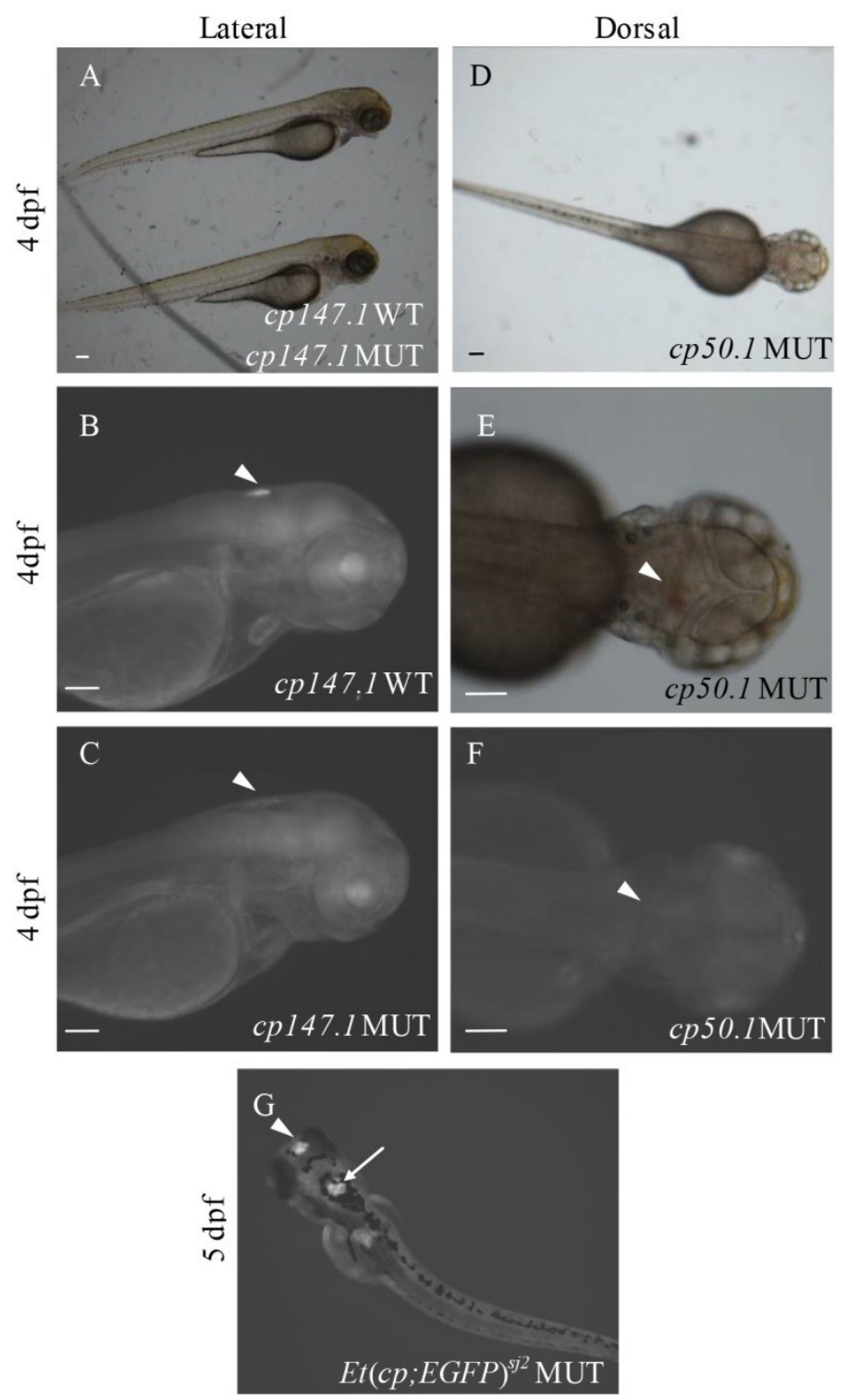

\section{Figure 3-1. Spontaneous Mutants with Normal Body Morphology but Absent/Abnormal CP.}

(A) Wild-type (top) and mutant (bottom) 4 dpf larvae from cp 147.1. (B) Lateral view of wild-type cp147.1 larvae. Arrowhead refers to the mCP. (C) Lateral view of mutant cp147.1 larvae. Arrowhead refers to presumptive $\mathrm{mCP}$ although it is absent at $4 \mathrm{dpf}$. (D) Dorsal brightfield view of cp50.1 mutant at $4 \mathrm{dpf}$. (E) Magnified brightfield image of cp50.1 mutant. Slight hemorrhage is present near the third ventricle (arrowhead). (F) Dorsal fluorescent image of $c p 50.1$ mutant. Arrowhead refers to presumptive mCP, although it is absent at $4 \mathrm{dpf}$. (G) Dorsal fluorescent image of a spontaneous larva at 5 dpf with a larger dCP (arrowhead) and $\mathrm{mCP}$ (arrow) but normal overall body morphology. Scale bars are $50 \mu \mathrm{m}$. 

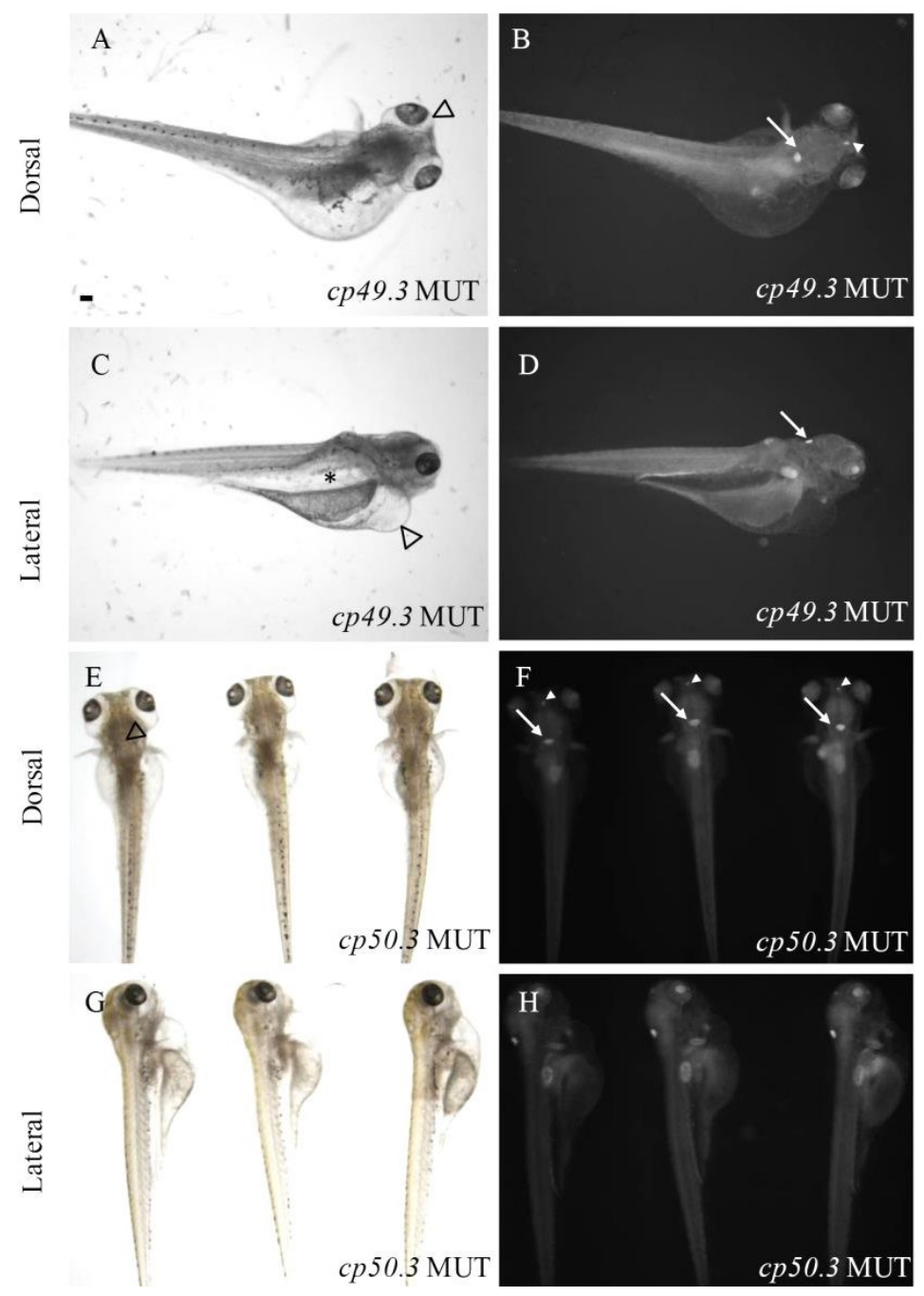

Figure 3-2. Spontaneous and Recessive Mutants with Normal CP Morphology.

Mutants have severe heart, eye and gut edema with little or no heartbeat and circulation and brain necrosis; however $\mathrm{dCP}$ and $\mathrm{mCP}$ appear normal in GFP intensity and $\mathrm{CP}$ morphology. Figure shows dorsal view in brightfield (A) and in fluorescence (B) of a spontaneous $E t(c p: E G F P)^{s j 2}$ larvae from $c p 49.3$ at 4 dpf. In (A), eye edema is represented by an open arrowhead. In (B), the $\mathrm{dCP}$ is represented by a filled arrowhead and an arrow is pointing at the $\mathrm{mCP}$. Lateral view in brightfield (C) and fluorescence (D) of cp49.3larvae at $4 \mathrm{dpf}$. In (C) heart edema is shown by an open arrowhead and an asterisk represents edema in the gut. In (D), the arrow represents the mCP. (E-H) shows larvae from cp50.3. We identified around 15\% mutant. (E) Dorsal brightfield image of cp50.3 mutant larvae at 4 dpf. (F) Dorsal fluorescent image of cp50.3 mutant larvae at 4 dpf. dCP (arrowhead) and mCP (arrow) shown for each mutant. (G) Lateral brightfield image of cp50.3 mutants at $4 \mathrm{dpf}$. $(\mathrm{H})$ Lateral fluorescent image of cp50.3 mutant larvae at 4 dpf. dCP, diencephalic choroid plexus; $\mathrm{mCP}$, myelencephalic choroid plexus. Scale bar is $50 \mu \mathrm{m}$. 
paragraphs were named based on the original $\mathrm{F}_{2}$ family and pair number within that family. No mutants were adult viable and most did not survive past $4 \mathrm{dpf}$, except for cp26.6 and cp105.2, which lived until $5 \mathrm{dpf}$ and $6 \mathrm{dpf}$, respectively. cp27.5 and cp44.10 mutants displayed similar phenotypes; however, complementation analysis showed that they were not in the same complementation group. On the basis of GFP intensity, epithelial localization, and overall CP morphology, we identified five classes: (1) mutants with reduced GFP expression, dispersed epithelial cells, and irregularly shaped CP; (2) mutants with normal GFP expression, small epithelial aggregates, and irregularly shaped CP; (3) mutants with normal GFP expression, small epithelial aggregates, and expanded $\mathrm{CP}$; (4) mutants with variable GFP expression, small epithelial aggregates, and expanded $\mathrm{CP}$; and (5) mutants with normal GFP expression, compact epithelial cells, and enlarged $\mathrm{CP}$.

To further classify these mutants, we analyzed brain ventricle morphology and transporter activity in the CP. To examine brain ventricle morphology, we injected a $40-\mathrm{kDa}$ fluorescein dextran into the ventricle of $E t(c p: E G F P)^{s j 2}$ mutants that were GFP negative by outcrossing the mutant lines to the wild-type TL strain, and selected based upon other phenotypic traits. Fluorescein transport was used as a secondary assay to measure transporter activity in the $\mathrm{CP}$ and overall permeability as described previously in Fluorescein Treatment on pg. 29.

Class I Mutants Have Reduced GFP Expression, Dispersed Epithelial Cells, and Irregularly Shaped CP. Class I comprised 2 mutant lines cp26.3 and cp140.2, which had reduced GFP and dispersed epithelial cells, resulting in an irregularly shaped mCP (Figure 3-3). The cp 26.3 mutants were observed starting at late 2 dpf to early 3 dpf. By 3 dpf, the mutants had a very faint heartbeat, no circulation, and developed heart edema. Although these mutants had relatively normal body morphology except for the absence of a swimbladder, they developed severe necrosis and did not survive beyond 3 dpf. The necrosis was localized primarily within the head region. Although the majority of the cp26.3 mutants exhibited hemorrhaging within the brain ventricle, there was no ventricle expansion. The mutants had normal hindbrain ventricle morphology, but ventricles were slightly smaller than those of wild-type larvae. They also had little to no midbrain and forebrain ventricles (Figure 3-3E-F").

Based upon additional phenotypes such as small head and eyes, cp140.2 mutants were identifiable at $2 \mathrm{dpf}$. CP development was monitored by time-lapse confocal imaging over a period of $48 \mathrm{~h}$, starting at $54 \mathrm{hpf}$ for wild-type larvae (see Appendix A. SupplementalMovie2) and cp140.2 mutants (see Appendix A. SupplementalMovie3). GFP intensity was reduced and there were defects in cell coalescence, resulting in a CP with discrete boundaries. The overall body morphology of cp 140.2 mutants was similar to that of wild-type larvae but smaller in size and lacked the swimbladder. The mutants developed heart edema but maintained a heartbeat and circulation until $4 \mathrm{dpf}$. They also exhibited brain necrosis by $4 \mathrm{dpf}$, but there was no hemorrhaging within the brain ventricle or ventricle expansion. cp140.2 mutants had normal hindbrain and broader 

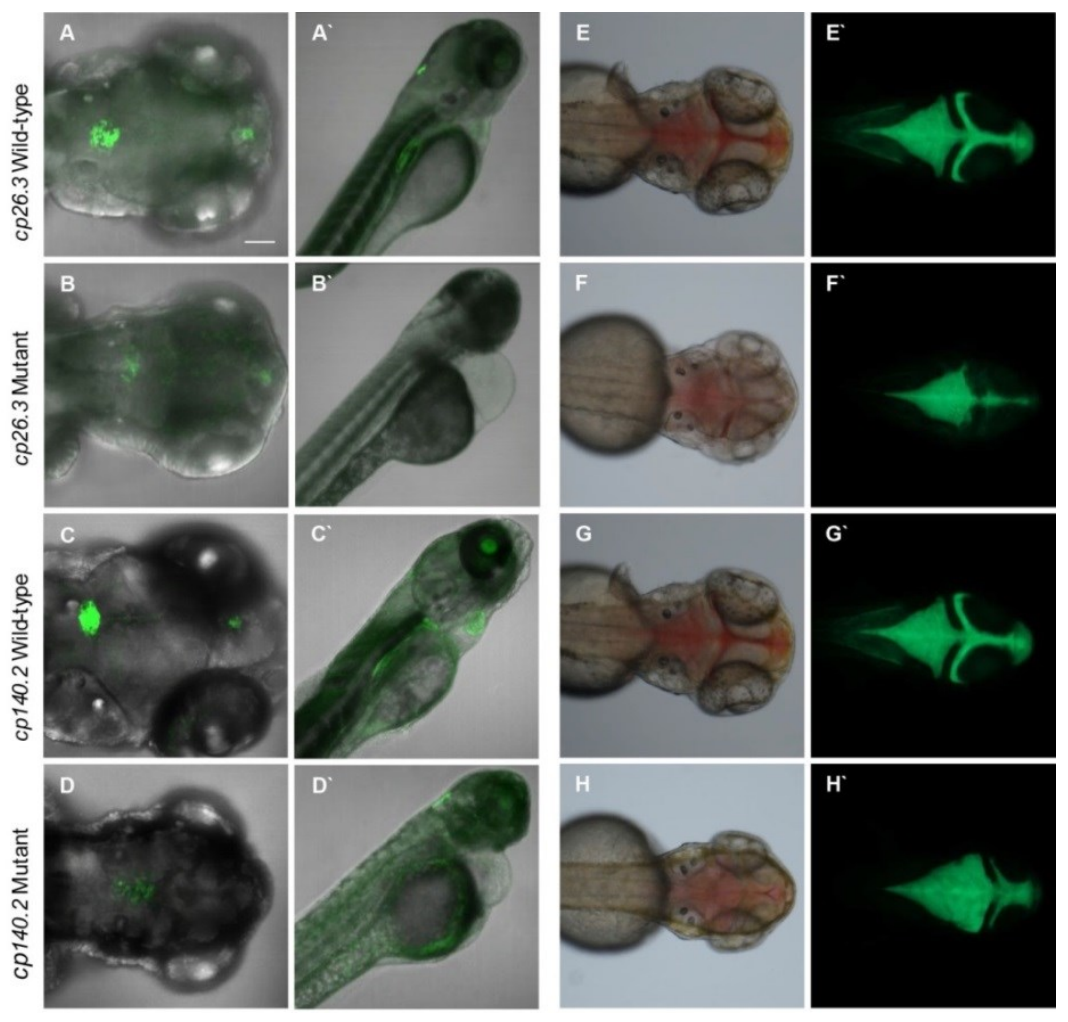

Figure 3-3. Class I Mutants Have Reduced GFP Expression, Dispersed Epithelial Cells, and Irregularly Shaped CP.

(A) Dorsal image of 3 dpf wild-type cp26.3 larvae at 20× magnification. (A') Lateral image of $3 \mathrm{dpf}$ wild-type cp26.3 larvae at 10× magnification. (B) Dorsal image of $3 \mathrm{dpf}$ mutant cp26.3 larvae at $20 \times$ magnification. (B') Lateral image of $4 \mathrm{dpf}$ mutant $c p 26.3$

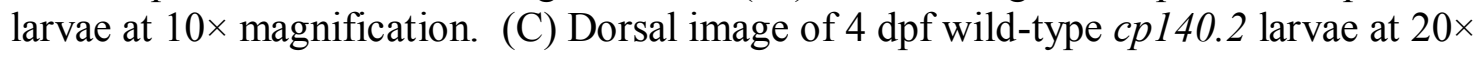
magnification. (C') Lateral image of 4 dpf wild-type cp140.2 larvae at 10× magnification. (D) Dorsal image of $4 \mathrm{dpf}$ mutant cp140.2 larvae at 20× magnification. (D') Lateral image of $4 \mathrm{dpf}$ mutant cp140.2 larvae at 10× magnification. (E) Bright-field image of 3 dpf wild-type $c p 26.3$ larvae after ventricle injection of the 40-kDa fluorescein dextran. (E') Fluorescent image of 3 dpf wild-type cp26.3 larvae after ventricle injection of the 40-kDa fluorescein dextran. (F) Bright-field image of 3 dpf mutant cp26.3 larvae after ventricle injection of the 40-kDa fluorescein dextran. (F') Fluorescent image of $3 \mathrm{dpf}$ mutant cp26.3 larvae after ventricle injection of the 40-kDa fluorescein dextran. (G) Bright-field image of 4 dpf wild-type cp 140.2 larvae after ventricle injection of the 40-kDa fluorescein dextran. (G') Fluorescent image of 4 dpf wild-type cp140.2 larvae after ventricle injection of the 40-kDa fluorescein dextran. $(\mathrm{H})$ Bright-field image of 4dpf mutant cp 140.2 larvae after ventricle injection of the 40-kDa fluorescein dextran. (H') Fluorescent image of $4 \mathrm{dpf}$ mutant cp140.2 larvae after ventricle injection of the $40-\mathrm{kDa}$ fluorescein dextran. Images in (A)-(D') were acquired by confocal microscopy, and those in $(\mathrm{E})-\left(\mathrm{H}^{\prime}\right)$ were acquired by fluorescent stereoscopy. Scale bar is $50 \mu \mathrm{m}$. 
midbrain ventricles, but less defined and slightly larger forebrain ventricles than that of wild-type larvae (Figure 3-3G-H').

Class II Mutants Have Normal GFP Expression, Small Epithelial Aggregates, and Irregularly Shaped CP. Class II comprised lines $c p 9.6$ and $c p 79.6$ (Figure 3-4). The $c p 9.6$ mutants were isolated at $2 \mathrm{dpf}$, based on the brain necrosis. Time-lapse imaging showed cell migration toward the dorsal midline to form the $\mathrm{mCP}$; however, the cells formed smaller aggregates, resulting in a misshapen CP (see

Appendix A SupplementalMovie4). These mutants also had a small head and eyes, no swimbladder, heart edema, and brain necrosis. By 4 dpf, they continued to have a heartbeat but slow circulation. Also, brain ventricle expansion or hydrocephaly occurred in some mutants by late $3 \mathrm{dpf}$ to $4 \mathrm{dpf}$. Ventricular morphology revealed no forebrain or midbrain ventricle (Figure 3-4E-F'). The hindbrain ventricle appeared smaller in size than that of wild-type larvae.

cp79.6 mutants were identified at approximately $2 \mathrm{dpf}$ and had a small head and eyes, no swimbladder, heart edema, and many mutants never hatched out of the chorion. By $4 \mathrm{dpf}$, the mutants had a very slow heartbeat, little to no circulation, brain necrosis, and hemorrhaging around the heart. Time-lapse imaging revealed that the epithelial cells were initially dispersed and similar to those of the cp140.2 mutants (see Appendix A SupplementalMovie5). Although the cells became more compact over time, the final CP had internal regions with no GFP expression, suggesting that the cells formed smaller aggregates rather than a compact structure. Although these mutants had a defect in $\mathrm{CP}$ development, overall brain ventricle morphology was normal, except for a larger midbrain ventricle as seen in cp140.2 (Figure 3-4G-H)

\section{Class III Mutants Have Normal GFP Expression, Small Epithelial}

Aggregates, and Expanded CP. Class III comprised 2 mutant lines $c p 79.8$ and $c p 151.2$, which had normal GFP expression but dispersed cell aggregates, resulting in an expanded CP (Figure 3-5). Both mutants had a small head and eyes, heart edema, no swimbladder, and brain necrosis. The cp 79.8 mutants were isolated by $2 \mathrm{dpf}$. By $4 \mathrm{dpf}$, mutants showed hemorrhaging in the heart and most had a decreased heartbeat and circulation. Brain ventricle morphology was relatively normal and resembled that of $c p 79.6$ mutants (Figure 3-5E-F").

The cp151.2 mutants were identified at approximately late $2 \mathrm{dpf}$ to $3 \mathrm{dpf}$. Although their overall body morphology was fairly normal but smaller in size, by $4 \mathrm{dpf}$, these mutants developed minor swelling around the head and occasionally exhibited hemorrhaging around the heart, but continued to have a heartbeat and circulation. $\mathrm{CP}$ development over time showed normal GFP intensity, but the initial localization of CPe was more expanded than that of wild-type larvae (see Appendix A.

SupplementalMovie6). Although the cells did coalesce, they tended to form smaller aggregates rather than migrating together to form a compact CP. Also, toward the end of the $48 \mathrm{~h}$ time course, cell death occurred within the epithelium. Although the $\mathrm{CP}$ was 

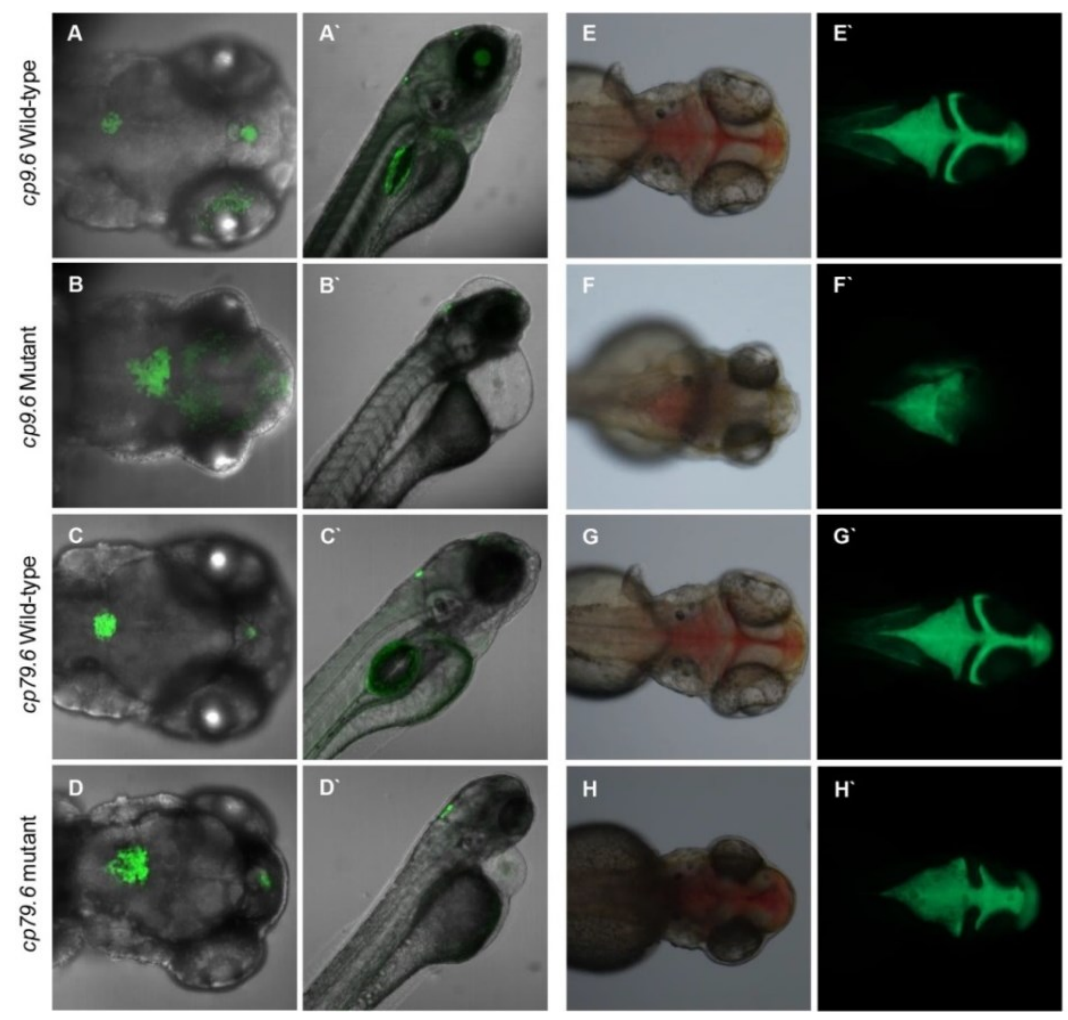

\section{Figure 3-4. Class II Mutants Have Normal GFP Expression, Small Epithelial Aggregates, and Irregularly Shaped CP.}

(A) Dorsal image of 4 dpf wild-type cp9.6 larvae at 20× magnification. (A') Lateral image of $4 \mathrm{dpf}$ wild-type $c p 9.6$ larvae at 10× magnification. (B) Dorsal image of $4 \mathrm{dpf}$ mutant cp9.6 larvae at $20 \times$ magnification. (B') Lateral image of 4 dpf mutant $c p 9.6$ larvae at $10 \times$ magnification. (C) Dorsal image of 4 dpf wild-type cp79.6 larvae at $20 \times$ magnification. (C') Lateral image of $4 \mathrm{dpf}$ wild-type cp79.6 larvae at $10 \times$ magnification. (D) Dorsal image of $4 \mathrm{dpf}$ mutant $c p 79.6$ larvae at 20× magnification. (D') Lateral image of $4 \mathrm{dpf}$ mutant $c p 79.6$ larvae at 10× magnification. (E) Bright-field image of $4 \mathrm{dpf}$ wild-type $c p 9.6$ larvae after ventricle injection of the 40-kDa fluorescein dextran. (E') Fluorescent image of $4 \mathrm{dpf}$ wild-type $c p 9.6$ larvae after ventricle injection of the $40-\mathrm{kDa}$ fluorescein dextran. (F) Bright-field image of 4 dpf mutant cp9.6 larvae after ventricle injection of the 40-kDa fluorescein dextran. (F') Fluorescent image of 4 dpf mutant cp9.6 larvae after ventricle injection of the 40-kDa fluorescein dextran. (G) Bright-field image of $4 \mathrm{dpf}$ wild-type $c p 79.6$ larvae after ventricle injection of the 40-kDa fluorescein dextran. $\left(\mathrm{G}^{\prime}\right)$ Fluorescent image of $4 \mathrm{dpf}$ wild-type cp79.6 larvae after ventricle injection of the 40-kDa fluorescein dextran. (H) Bright-field image of 4 dpf mutant cp79.6 larvae after ventricle injection of the $40-\mathrm{kDa}$ fluorescein dextran. $\left(\mathrm{H}^{\prime}\right)$ Fluorescent image of the $4 \mathrm{dpf}$ mutant $c p 79.6$ larvae after ventricle injection of the 40-kDa fluorescein dextran. Images in (A)-(D') were acquired by confocal microscopy, and those in (E)-(H') were acquired by fluorescent stereoscopy. 


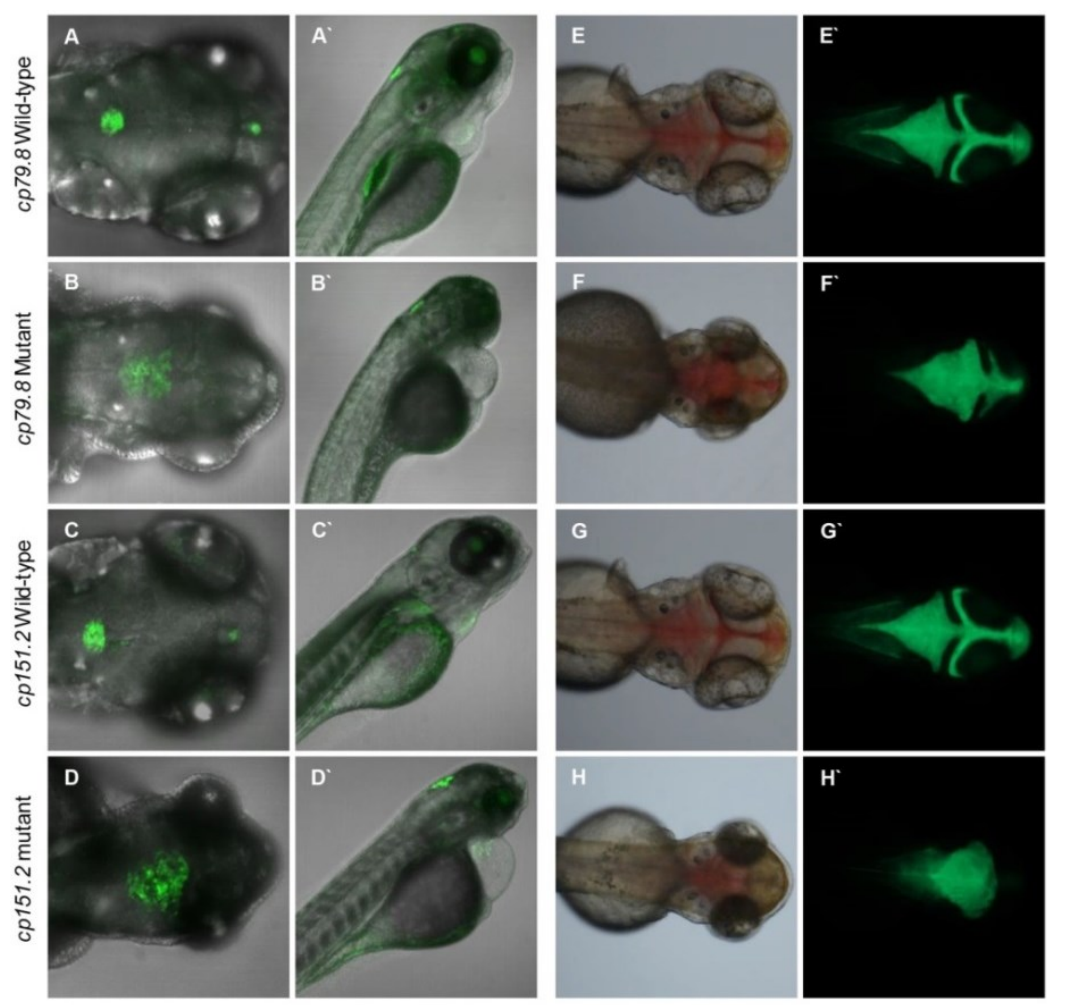

Figure 3-5. Class III Mutants Have Normal GFP Expression, Small Epithelial Aggregates, and Expanded CP.

(A) Dorsal image of 4 dpf wild-type cp79.8 larvae at 20× magnification. (A') Lateral image of $4 \mathrm{dpf}$ wild-type $c p 79.8$ larvae at $10 \times$ magnification. (B) Dorsal image of $4 \mathrm{dpf}$ mutant $c p 79.8$ larvae at $20 \times$ magnification. (B') Lateral image of $4 \mathrm{dpf}$ mutant $c p 79.8$ larvae at $10 \times$ magnification. (C) Dorsal image of $4 \mathrm{dpf}$ wild-type cp151.2 larvae at 20× magnification. (C') Lateral image of 4 dpf wild-type cp151.2 larvae at 10× magnification. (D) Dorsal image of 4 dpf mutant cp151.2 larvae at 20× magnification. (D') Lateral image of $4 \mathrm{dpf}$ mutant cp151.2 larvae at 10× magnification. (E) Bright-field image of 4 dpf wild-type $c p 79.8$ larvae after ventricle injection of the $40-\mathrm{kDa}$ fluorescein dextran. (E') Fluorescent image of 4 dpf wild-type $c p 79.8$ larvae after ventricle injection of the $40-\mathrm{kDa}$ fluorescein dextran. (F) Bright-field image of $4 \mathrm{dpf}$ mutant $c p 79.8$ larvae after ventricle injection of the $40-\mathrm{kDa}$ fluorescein dextran. (F') Fluorescent image of $4 \mathrm{dpf}$ mutant cp79.8 larvae after ventricle injection of the $40-\mathrm{kDa}$ fluorescein dextran. (G) Bright-field image of $4 \mathrm{dpf}$ wild-type cp151.2 larvae after ventricle injection of the 40-kDa fluorescein dextran. (G') Fluorescent image of 4 dpf wild-type cp151.2 larvae after ventricle injection of the 40-kDa fluorescein dextran. (H) Bright-field image of 4 dpf mutant cp 151.2 larvae after ventricle injection of the 40-kDa fluorescein dextran. (H') Fluorescent image of 4 dpf mutant cp 151.2 larvae after ventricle injection of the $40-\mathrm{kDa}$ fluorescein dextran. Images in (A)-(D') were acquired by confocal microscopy, and those in $(\mathrm{E})-\left(\mathrm{H}^{\prime}\right)$ were acquired by fluorescent stereoscopy. 
expanded, there appeared to be one large ventricle near the hindbrain rather than distinct midbrain-hindbrain boundaries. There was also very low accumulation of $40-\mathrm{kDa}$ fluorescein within the forebrain ventricle (Figure 3-5G-H).

\section{Class IV Mutants Have Variable GFP Expression, Small Epithelial} Aggregates, and Expanded CP. Class IV comprised 2 mutant lines $c p 27.5$ and $c p 44.10$ (Figure 3-6). The GFP expression in these mutants varied between cells having normal GFP intensity and those with lower expression. Similar to Class III mutants, $c p 27.5$ and cp44.10 also formed clusters of cells rather than a single compact structure, which resulted in an expanded CP (Figure 3-6). For $c p 27.5, \mathrm{CP}$ formation over time showed a pattern of development similar to that of $c p 151.2$; however, GFP intensity varied throughout the CP (see Appendix A. SupplementalMovie7). Both cp27.5 and cp44.10 were identified at $2 \mathrm{dpf}$ and displayed small heads, small eyes, and no swimbladder. Other unique features were a significantly smaller and arched body, expanded ventricles or hydrocephaly by $3 \mathrm{dpf}$, and tails that curved to the side by $4 \mathrm{dpf}$. Additional phenotypes at $4 \mathrm{dpf}$ included brain necrosis and heart edema. The mutants had a heartbeat at $4 \mathrm{dpf}$, but little to no circulation. Also, there was occasional hemorrhaging in the brain ventricle for $c p 27.5$ and near the heart for $c p 44.10$. These mutants exhibited the most severe defects in terms of brain ventricle morphology among all the classes. $c p 27.5$ had one large ventricle with no clearly defined forebrain, midbrain, and hindbrain boundaries (Figure 3-6E-F"). c $p 44.10$ had a smaller, irregularly shaped hindbrain, but the $40-\mathrm{kDa}$ fluorescein could not penetrate into the midbrain or forebrain ventricles (Figure 3-6G-H').

Class V Mutants Have Normal GFP Expression, Compact Epithelial Cells, and Enlarged CP. Class V comprising 2 mutant lines cp26.6 and cp105.2, exhibited the least severe CP phenotype, and survived longer than other mutant classes. The CP appeared relatively normal compared with that of wild-type larvae, although it was larger in size (Figure 3-7). Also, both mutants had a rounder head and a protruding lower jaw. The $c$ p 26.6 mutant was isolated by 4 dpf. Mutants had no swimbladder and had edema in the heart, eyes, and near the presumptive swimbladder. They also had a faint heartbeat and circulation by $4 \mathrm{dpf}$. For cp 26.6 mutants at $4 \mathrm{dpf}$, the brain ventricle morphology was normal, but more closely resembled wild-type larvae at $3 \mathrm{dpf}$ (Figure 3-7E-F'). Also, cp105.2 mutants had a slightly smaller hindbrain ventricle compared to wild-type (Figure 3-7G-H). The cp 105.2 mutants were isolated by late $4 \mathrm{dpf}$ to $5 \mathrm{dpf}$, based on the absence of a swimbladder. The CP initially appeared normal, but became larger as the mutants developed severe heart and eye edema. Though the swimbladder was absent, severe edema was localized to this region. The heartbeat and circulation began to diminish by late $5 \mathrm{dpf}$ to early $6 \mathrm{dpf}$. 

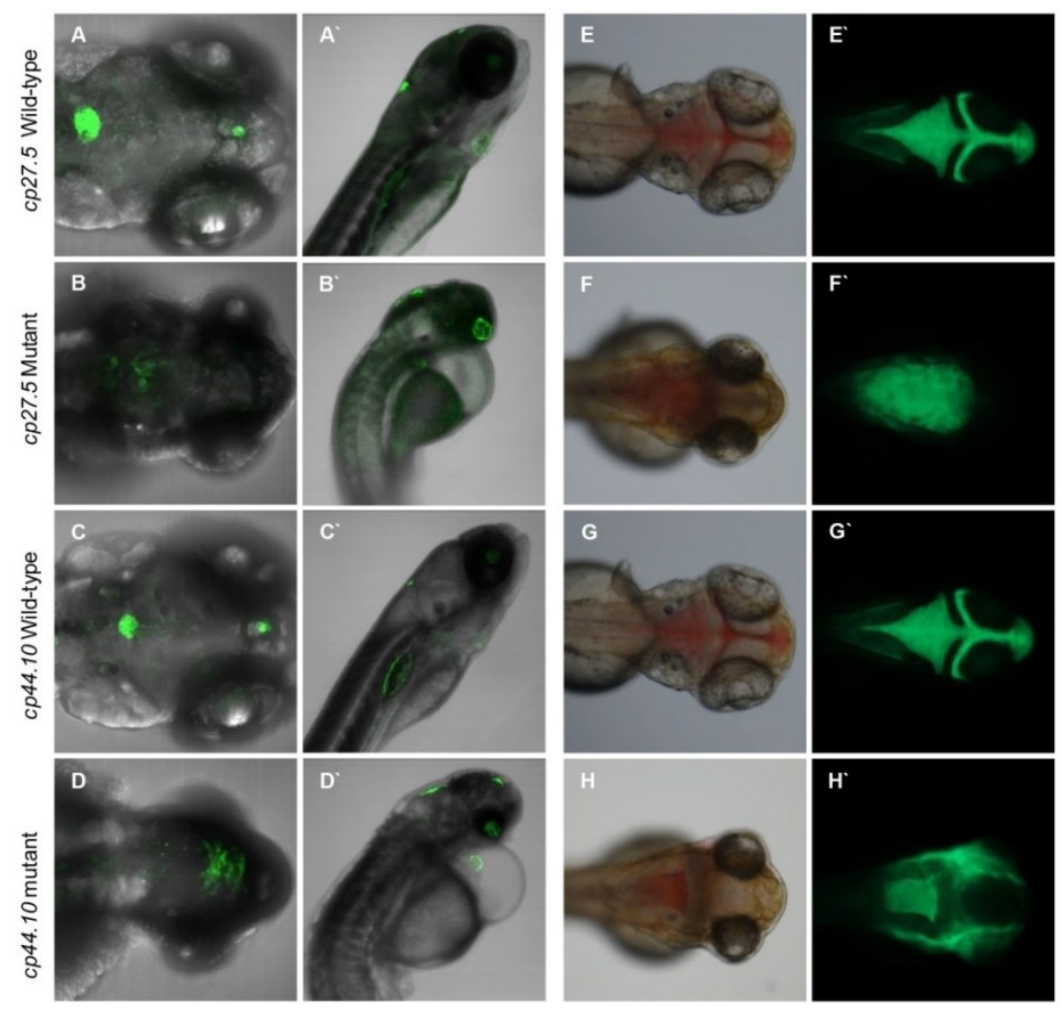

Figure 3-6. Class IV Mutants Have Variable GFP Expression, Small Epithelial Aggregates, and Expanded CP.

(A) Dorsal image of 4 dpf wild-type cp27.5 larvae at 20× magnification. (A') Lateral image of $4 \mathrm{dpf}$ wild-type $c p 27.5$ larvae at 10× magnification. (B) Dorsal image of $4 \mathrm{dpf}$ mutant cp27.5 larvae at $20 \times$ magnification. (B') Lateral image of 4 dpf mutant cp27.5 larvae at $10 \times$ magnification. (C) Dorsal image of $4 \mathrm{dpf}$ wild-type $c p 44.10$ larvae at $20 \times$ magnification. (C') Lateral image of $4 \mathrm{dpf}$ wild-type cp44.10 larvae at $10 \times$ magnification. (D) Dorsal image of 4 dpf mutant cp44.10 larvae at 20× magnification. (D') Lateral image of $4 \mathrm{dpf}$ mutant cp 44.10 larvae at 10× magnification. (E) Bright-field image of 4 dpf wild-type $c p 27.5$ larvae after ventricle injection of the 40-kDa fluorescein dextran. (E') Fluorescent image of 4 dpf wild-type $c p 27.5$ larvae after ventricle injection of the 40-kDa fluorescein dextran. (F) Bright-field image of $4 \mathrm{dpf}$ mutant cp27.5 larvae after ventricle injection of the $40-\mathrm{kDa}$ fluorescein dextran. (F') Fluorescent image of $4 \mathrm{dpf}$ mutant cp27.5 larvae after ventricle injection of the 40-kDa fluorescein dextran. (G) Bright-field image of $4 \mathrm{dpf}$ wild-type cp44.10 larvae after ventricle injection of the 40-kDa fluorescein dextran. (G') Fluorescent image of 4 dpf wild-type cp44.10 larvae after ventricle injection of the 40-kDa fluorescein dextran. (H) Bright-field image of 4 dpf mutant cp44.10 larvae after ventricle injection of the 40-kDa fluorescein dextran. (H') Fluorescent image of $4 \mathrm{dpf}$ mutant cp44.10 larvae after ventricle injection of the $40-\mathrm{kDa}$ fluorescein dextran. Images in (A)-(D') were acquired by confocal microscopy, and those in $(\mathrm{E})-\left(\mathrm{H}^{\prime}\right)$ were acquired by fluorescent stereoscopy. 

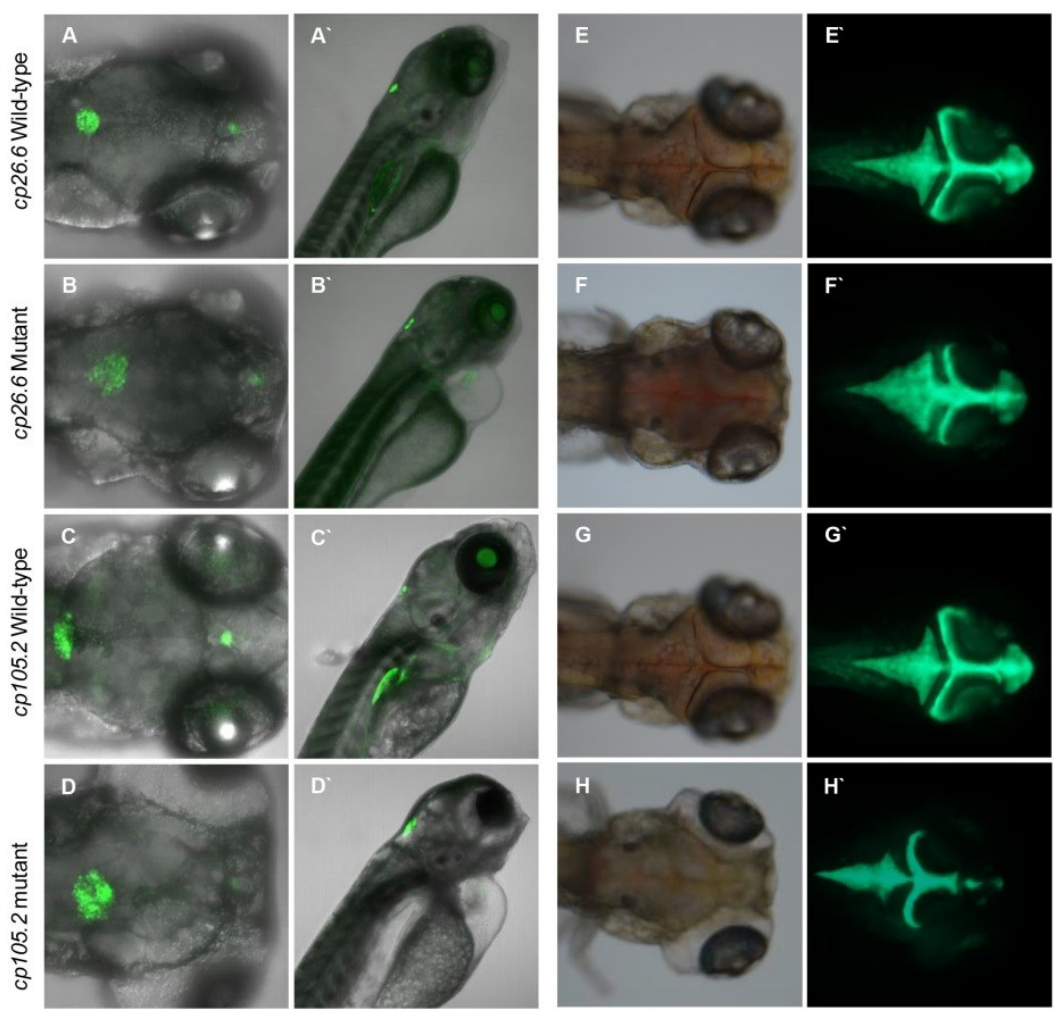

Figure 3-7. Class V Mutants Have Normal GFP Expression, Compact Epithelial Cells, and Enlarged CP.

(A) Dorsal image of 4 dpf wild-type cp26.6 larvae at 20× magnification. (A') Lateral image of $4 \mathrm{dpf}$ wild-type cp26.6 larvae at 10× magnification. (B) Dorsal image of $4 \mathrm{dpf}$ mutant cp26.6 larvae at 20× magnification. (B') Lateral image of 4 dpf mutant cp26.6 larvae at $10 \times$ magnification. (C) Dorsal image of $4 \mathrm{dpf}$ wild-type cp 105.2 larvae at 20× magnification. (C') Lateral image of 4 dpf wild-type cp105.2 larvae at 10× magnification. (D) Dorsal image of 4 dpf mutant cp105.2 larvae at 20× magnification. (D') Lateral image of 4dpf mutant cp105.2 larvae at 10× magnification. (E) Bright-field image of 4 dpf wild-type cp26.6 larvae after ventricle injection of the 40-kDa fluorescein dextran. (E') Fluorescent image of 4 dpf wild-type cp26.6 larvae after ventricle injection of the 40-kDa fluorescein dextran. (F) Bright-field image of $4 \mathrm{dpf}$ mutant cp26.6 larvae after ventricle injection of the $40-\mathrm{kDa}$ fluorescein dextran. (F') Fluorescent image of $4 \mathrm{dpf}$ mutant cp26.6 larvae after ventricle injection of the 40-kDa fluorescein dextran. (G) Bright-field image of 4 dpf wild-type cp 105.2 larvae after ventricle injection of the 40-kDa fluorescein dextran. (G') Fluorescent image of 4 dpf wild-type cp105.2 larvae after ventricle injection of the 40-kDa fluorescein dextran. (H) Bright-field image of 4 dpf mutant cp 105.2 larvae after ventricle injection of the 40-kDa fluorescein dextran. (H') Fluorescent image of $4 \mathrm{dpf}$ mutant cp 105.2 larvae after ventricle injection of the $40-\mathrm{kDa}$ fluorescein dextran. Images in (A)-(D') were acquired by confocal microscopy, whereas those in $(E)-\left(H^{\prime}\right)$ were acquired by fluorescent stereoscopy. 


\section{Fluorescein Assay to Determine CP Transport}

To determine if the CP could transport fluorescein in the mutants, GFP negative larvae were treated at $3 \mathrm{dpf}$ with $50 \mu \mathrm{M}$ fluorescein as described in the characterization study. For $c p 26.3$, fluorescein tended to accumulate toward the anterior part of the brain and no fluorescein accumulated within the CP (Figure 3-8E). Fluorescein did not accumulate in the $\mathrm{CPe}$ in the cp140.2 mutant, but was localized throughout the brain ventricle. These mutants also had a higher overall fluorescein uptake than wild-type. (Figure 3-8M). cp9.6 mutants also exhibited a higher overall uptake of fluorescein than wild-type and concentrated within the brain ventricle, gut, and heart. There was no distinct accumulation within the CP (Figure 3-8F). For $c p 79.6$, the overall uptake of fluorescein was comparable to wild-type. We observed very faint punctate accumulate of fluorescein within the general area of the CP indicating possible transport (Figure 3-8N).

Class III mutants (cp79.8 and $c p 151.2$ ) had increased uptake of fluorescein compared to wild-type and both had accumulation within the brain ventricle, gut, and heart. No fluorescein accumulated within the CPe (Figure 3-8G and 3-80). A similar phenotype was observed in Class IV mutants (cp27.5 and cp44.10) in that they also had increased uptake of fluorescein concentrated within the brain ventricle, gut, and heart, but no localization within CPe (Figure 3-8H and 3-8P). We were not able conclude whether Class V mutants (cp26.6 and cp105.2) demonstrated transport activity using this assay because of the inability to confidently identify GFP negative mutants at $3 \mathrm{dpf}$ and $4 \mathrm{dpf}$. Once we identified mutants by late $4 \mathrm{dpf}-5 \mathrm{dpf}$, fluorescein was rapidly cleared by the gut and was not distributed throughout the embryo (data not shown).

\section{Discussion}

To characterize mutants in our study, we examined not only GFP expression in the CP but also ventricle morphology and transporter activity. Previous studies have grouped zebrafish brain ventricle mutants into four categories: midline separation defects, reduced ventricle size, midbrain and hindbrain abnormalities, and absence of lumen inflation (Lowery et al., 2009). Because none of our CP mutants exhibited a phenotype at $24 \mathrm{hpf}$, we did not expect to see defects in midline separation as described in Lowery et al. (2009) (Lowery et al., 2009). CP mutants such as cp79.6 and cp140.2 have a brain ventricle that closely resembles a normal larva at $2 \mathrm{dpf}$ (Lowery et al., 2007), indicating that these mutants may have a developmental delay and not necessarily a brain ventricle defect. Also, cp 140.2 mutants at $3 \mathrm{dpf}$ are similar to wild-type larvae at $2 \mathrm{dpf}$ in that fluorescein accumulated within the brain ventricle rather than concentrating within $\mathrm{CPe}$ as it does normally on day 3. Many mutants exhibited an increased overall uptake of fluorescein, which may indicate defects in the skin epithelial barriers as well resulting in greater permeability to absorb small molecules from their surroundings.

While mutants exhibit little to no heart beat and circulation when screened on day 4, as described in Table 3- 2, the majority of mutants do have a strong heart beat and rapid circulation indistinguishable from wild-type prior to this time point. The initial 

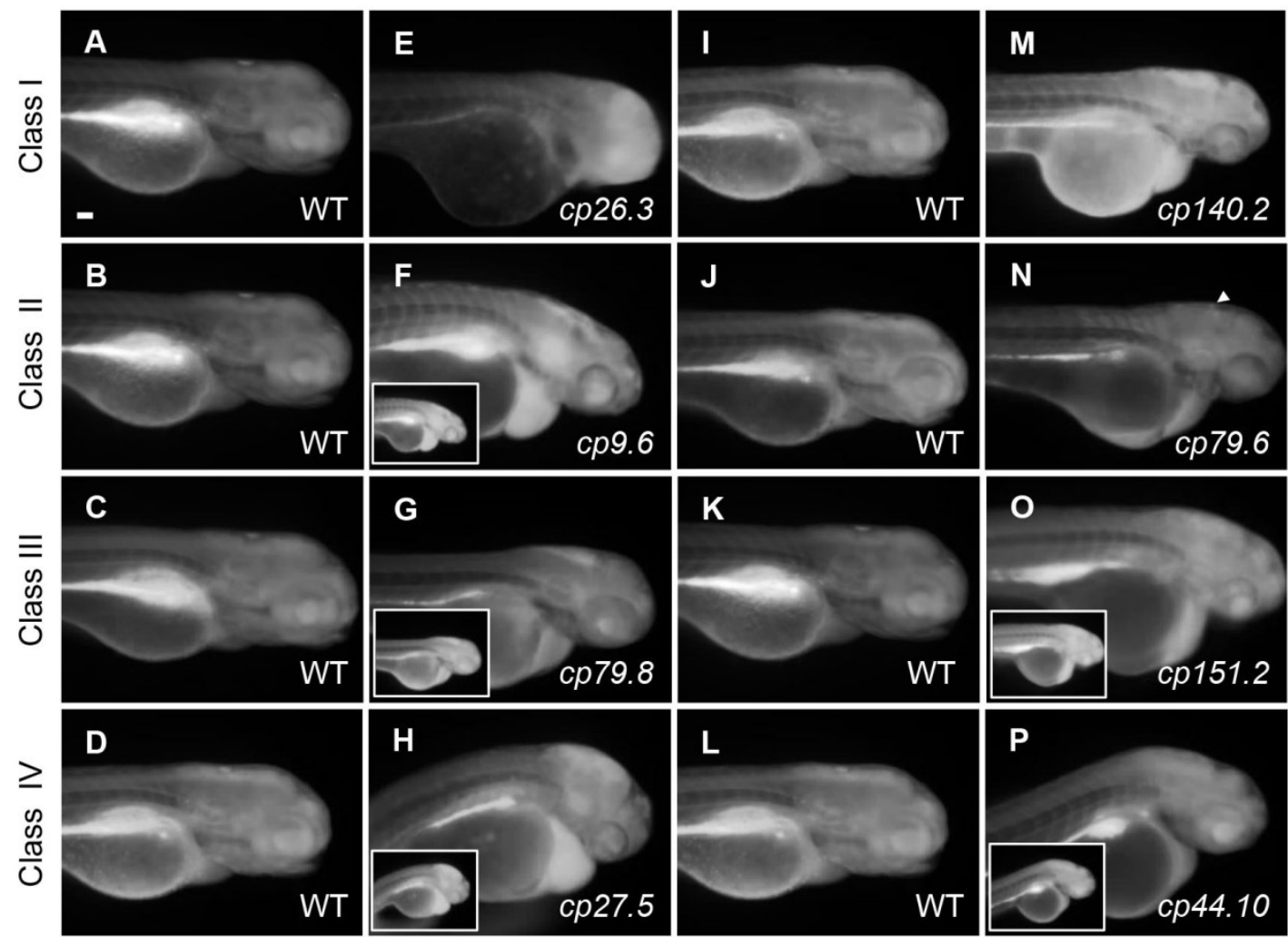

\section{Figure 3-8. Fluorescein Assay Determines CP Transport Activity in Mutants.}

(A-D) and (I-L) Wild-type larvae treated at $3 \mathrm{dpf}$ with $50 \mu \mathrm{M}$ fluorescein. (E) $c p 26.3$ mutant at $3 \mathrm{dpf}$. (F) $c p 9.6$ mutant at $3 \mathrm{dpf}$. Inset image is acquired at the same exposure to the corresponding wild-type in (B). (G) cp79.8 mutant at $3 \mathrm{dpf}$. Inset image is acquired at the same exposure to the corresponding wild-type in $(\mathrm{C}) .(\mathrm{H}) c p 27.5$ mutant at $3 \mathrm{dpf}$. Inset image is acquired at the same exposure to the corresponding wild-type in (D). (M) cp140.2 mutant at 3 dpf. (N) cp79.6 mutant at 3 dpf. Punctate accumulation of fluorescein in the brain ventricle is shown with an arrowhead. (O) cp151.2 mutant at 3 dpf. Inset image is acquired at the same exposure to the corresponding wild-type in $(\mathrm{K})$. (P) cp 44.10 mutant at $3 \mathrm{dpf}$. Inset image is acquired at the same exposure to the corresponding wild-type in (L). The larger images for F, G, H, O, and P were taken at a lower exposure compared to the corresponding wild-type in order to visualize where the fluorescein was concentrating throughout the larvae. Scale bar is $50 \mu \mathrm{m}$. 
onset of circulation loss describes when we first observed mutants with decreased or no circulation. Not all of the mutants had circulation loss at the same time, but the onset describes when we observed the first mutant from a spawn having no circulation. Because most of the mutants began to have decreased heartbeat and circulation at $4 \mathrm{dpf}$, after the $\mathrm{CP}$ has already developed, we conclude that other factors are regulating $\mathrm{CP}$ formation. However, we cannot rule out that circulation is required for $\mathrm{CP}$ maintenance. We also do not attribute the lack of blood flow to abnormal CP development because we identified spontaneous mutants with a normal CP but no circulation as shown in Figure 3-2. Because many mutants continued to have a heartbeat and circulation at $3 \mathrm{dpf}$, which are factors essential for brain ventricle expansion (Lowery and Sive, 2005), we did not expect ventricle expansion defects in these mutants. While ventricle morphology varied between lines, all had expanded ventricles.

The mutants identified in our screen will provide the tools necessary to investigate several genes associated with CP development and barrier function. After isolating defective genes in these mutants, we can begin to define the correlation between the mutated gene and the phenotypic abnormalities we observed. Although we identified mutants with brain necrosis, we do not contribute necrosis alone is a factor responsible for abnormal $\mathrm{CP}$ development and believe that these processes can be unrelated. This is supported as shown in Figure 3-2, where larvae with brain necrosis developed a normal CP. Furthermore, for the mutants we characterized further, we did not observe the onset of necrosis until after the CP had already started to develop for many of the lines, or if necrosis was present, it was not near the mCP itself. We also found larvae that had normal morphology overall, but a severely abnormal or absent $\mathrm{CP}$ as shown in Figure 3-1 indicating again that necrosis is not the sole mechanism responsible for abnormal $\mathrm{CP}$ development and that specific pathways exist in regulation its formation and function. We expect some of the genes we identify will be specifically related to a CP defect rather than pleiotropy that results in an overall severely abnormal animal. Pleiotropy is where a mutation in one gene can cause multiple phenotypes (Lobo, 2008)

Recent discoveries have shed light on the importance of the CP and its contribution to disease. For example, transcriptome analysis during early development revealed that the $\mathrm{CP}$ expresses numerous genes essential for $\mathrm{CP}$ function, including those for tight junctions, transporters, and metabolic function (Liddelow et al., 2012), protecting the brain from potentially harmful insults (Kratzer et al., 2013). The mutants identified in our study will be used to identify genes essential for maintaining epithelial barrier integrity as well as genes essential for initial barrier formation. Reverse genetics studies have shown that transcription factors such as Otx2 aid in CP development and maintenance (Johansson et al., 2013). Also, augurin, a protein encoded by the esophageal cancer related gene-4 (Ecrg4) and thought to act as a tumor suppressor, has a higher expression in the CP than other tissues. Interestingly, morpholino knockdown of Ecrg4 in zebrafish results in increased CNS cell proliferation and hydrocephaly (Gonzalez et al., 2011). Additional studies in zebrafish showed that the clusterin gene, which encodes a secreted glycoprotein that aids in aggregation and contributes to Alzheimer's disease by co-localizing with plaques (Thambisetty et al., 2010), is similar to its human counterpart and is expressed in the $\mathrm{dCP}$ and $\mathrm{mCP}$ (Jiao et al., 2011). Demonstrating similarities in 
gene expression in zebrafish and human $\mathrm{CP}$ is important when considering the use of zebrafish as a disease model for CP-related conditions. Ultimately, the genes discovered from these mutants will provide a better understanding of the genetic and molecular mechanisms involved in CP formation and function and provide new insights into how these normal processes become altered in disease.

\section{Conclusion}

Forward genetic screening is a powerful tool to uncover genetic processes essential for development and/or maintenance of specific cell types, tissues, or overall morphology of an organism. We have performed the first forward genetic screen to identify mutants with CP defects using GFP as a readout to observe its development over time. Using this unbiased approach, we discovered 10 recessive mutant lines with CP phenotypes. While these mutants also exhibit other abnormalities, we have provided evidence that specific pathways essential for $\mathrm{CP}$ development do exist by discovering mutants with normal morphology and no $\mathrm{CP}$ or mutants with severe abnormalities, but normal CP development. Future studies will investigate whether the genes responsible for the recessive mutant phenotypes are specific to the $\mathrm{CP}$ and play a role in its development, function, and maintenance. 


\section{CHAPTER 4. POSITIONAL CLONING AND WHOLE-EXOME SEQUENCING (WES) IDENTIFIES SART1 AS DEFECTIVE GENE IN CHOROID PLEXUS MUTANT}

\section{Introduction}

The spliceosome consists of a complex network of proteins and small nuclear ribonucleoproteins called snRNPs that remove introns from precursor messenger RNA (pre-mRNA) to form mature mRNA. Although the process of splicing was discovered more than thirty years ago (Matera and Wang, 2014), the mechanisms of spliceosome assembly and splicing regulation are not well understood. Over 100 proteins have been identified in regulating spliceosome assembly or function (Jurica and Moore, 2003). Understanding the role these proteins play in splicing is important because a number of disorders, such as retinitis pigmentosa (RP) (Tanackovic et al., 2011), amyotrophic lateral sclerosis, spinal muscular atropy (Tsuiji et al., 2013), and chronic lymphocytic leukemia (Rozovski et al., 2013) can occur as a result of mutations in splicing factors.

Here we present a mutation in one of these proteins, squamous cell carcinoma antigen recognized by $T$-cells (sart1), which was discovered from the forward genetic screen in zebrafish identifying mutants in CP development. This mutant line previously referred to as cp27.5, not only has defects in CP development but also ventricle expansion, eye formation, and CNS and epithelial barrier permeability. Mutants are embryonic lethal dying around late day 4 . We genetically mapped the mutation from cp27.5 using bulked-segregant analysis and identified a critical interval along Chromosome 21 . We identified the gene as sartl using WES, which has recently been utilized as an effective and time-efficient strategy to identify mutations in lines discovered from zebrafish forward genetic screens (Kettleborough et al., 2013; Ryan et al., 2013). Using this approach, we discovered that sartl has a point mutation containing a $\mathrm{G}$ to A transition at the donor site of exon 12 . We also confirmed by mRNA rescue that sartl is the gene responsible for the phenotypes we described previously in cp 27.5 mutants.

\section{Materials and Methods}

\section{Genomic DNA Isolation}

Adult zebrafish were anesthetized in $0.02 \%$ tricaine (Sigma-Aldrich) and tail clipped. As the fish recovered in system water, the tail clip was immediately immersed into $50 \mu \mathrm{L}$ digest buffer $(0.2 \mathrm{M} \mathrm{NaCl}, 10 \mathrm{mM}$ Tris $8.0,5 \mathrm{mM}$ EDTA, $0.1 \% \mathrm{SDS}, 0.1$ $\mathrm{mg} / \mathrm{ml}$ Proteinase K). Samples were incubated overnight at $50^{\circ}$. After brief centrifugation, $450 \mu \mathrm{L}$ of $100 \%$ ethanol (EtOH) was added. Samples were centrifuged at $21,130 \mathrm{~g}$ or $15,000 \mathrm{rpm}$ for $10 \mathrm{~min}$, the supernatant was removed, and $500 \mu \mathrm{L}$ of $70 \%$

EtOH added to wash the pellet. Samples were centrifuged at $21,130 \mathrm{~g}$ or $15,000 \mathrm{rpm}$ for 
5 min. After removing the supernatant, the samples were stored at room temperature for about 30 min to evaporate excess EtOH. The DNA pellets were resuspended with $50 \mu \mathrm{L}$ of TE buffer (10 mM Tris and $1 \mathrm{mM}$ EDTA, $\mathrm{pH} 8.0)$.

For zebrafish larvae, wild-type and mutants were anesthetized, separated into 1.5 $\mathrm{mL}$ eppendorf tubes, and immersed in $1 \mathrm{~mL} \mathrm{100 \%} \mathrm{methanol} \mathrm{(MeOH)} \mathrm{(Sigma-Aldrich).}$ Samples were stored at $-20^{\circ}$ overnight. The following day, individual wild-type and mutant larvae were transferred into $0.5 \mathrm{~mL}$ tubes and the excess $\mathrm{MeOH}$ removed. Once all the $\mathrm{MeOH}$ evaporated, $10 \mu \mathrm{L}$ of digest buffer (same as above) was added to each tube. Samples were incubated overnight at $50^{\circ}$. DNA was isolated as described above for adults except the volume of $100 \% \mathrm{EtOH}$ and $70 \% \mathrm{EtOH}$ was $180 \mu \mathrm{L}$ and the volume of TE buffer was $25 \mu \mathrm{L}$.

\section{Positional Cloning}

After identifying a mutant line, the $E t(c p: E G F P)^{s j 2} \mathrm{~F}_{2}$ heterozygous parents were each outcrossed to the polymorphic TL strain. From this outcross, heterozygous hybrid adults were isolated based their offspring with the recessive mutant phenotype. The wild-type and mutant offspring from these heterozygous hybrids were collected and their DNA was extracted (see above). A genome scan using 192 polymorphic Z-markers was performed with DNA pooled from 30 wild-type and 30 mutant larvae as previously described (Muto et al., 2005). Wild-type and mutant DNA was amplified using the Accuprime Taq DNA Polymerase System (Invitrogen). The resulting PCR products were analyzed for genetic linkage by agarose gel electrophoresis. For fine genetic mapping, individual wild-type and mutant DNA was amplified using Platinum PCR Supermix (Invitrogen). Z-markers used for genetic mapping were acquired from the zebrafish genome database at http://www.ensembl.org/index.html (Flicek et al., 2014). Additional markers were designed by examining the Ensembl genome browser for polymorphic regions in the genome (Flicek et al., 2014).

\section{Whole-Exome Sequencing and Analysis}

cp27.5 wild-type and mutant larvae (20 each) were collected in $\mathrm{MeOH}$ at $3 \mathrm{dpf}$. The 20 larvae were generated from 3 different heterozygous parents. DNA was extracted and using the MagAttract HMW DNA kit (Qiagen) following the manufacturer's instructions. Values for DNA concentration and purity were obtained by a ND-1000 NanoDrop Spectrophotometer and Qubit assay. Concentration was adjusted to the required $3 \mu \mathrm{g} / 120 \mu \mathrm{L}$ for sequence capture. Sequence capture and sequencing was done by the Genome Sequencing Facility Hartwell Center for Bioinformatics and Biotechnology at St. Jude Children's Research Hospital. Paired end 100 cycle sequencing was run on a HiSeq 2500 using TruSeq SBS v3 chemistry according to the manufacturer's instructions (Illumina, San Diego, CA.). From the sequencing results, we had coverage of all exons with at least $20 \mathrm{x}$ coverage for $80 \%$ of the regions and average coverage of the target regions was even higher. 
From the raw sequencing data, the quality control, read mapping and variant calling were performed using CLC Genomic Workbench v6.5 (CLC Bio, Denmark). In brief, the reads were trimmed against the sequencing adapters and only reads with a sequencing quality greater than 20 and a read length greater than 50 bp were retained. The filtered reads were then aligned to the zebrafish reference genome sequence (Zv9 assembly, 2010), and the lists of single-nucleotide variants (SNV) and indels were generated. To identify the causative mutation responsible for the phenotype, we compared the variants of mutant with those of wild type, and a region enriched with mutant-specific homozygosities was observed at a peak of homozygosity scores defined by the percentage of mutant-specific homozygous variants within a 1-Mb window across the genome. A candidate mutation was further pinpointed by screening the region for nonsense and essential splice mutations. A diagram of the WES workflow and analysis is represented in Figure 4-1.

\section{sart1 RNA Rescue}

cDNA from 3 dpf wild-type larvae was amplified using the AccuPrime Taq DNA Polymerase System to produce a $\sim 2.5 \mathrm{~kb}$ fragment of sart 1 open reading frame (ORF). After analyzing the PCR product on a 1\% gel, the product was cut out of the gel and purified using the QIAquick Gel Extraction kit (Qiagen). Molecular cloning was done to insert the sartl fragment into the PCRII TOPO vector using the PCRII TOPO TA cloning kit dual promoter (Life Technologies) and transformed into TOP 10F' chemically competent E. coli (Invitrogen). Plasmid DNA was purified using the DNA Miniprep kit (Qiagen). The plasmid was then cut using EcoRV and Spe 1 restriction enzymes (New England BioLabs) to release the sart1 fragment. The $\mathrm{pCS}^{+}$vector was cut using SnaB1 and $X b a 1$ and a ligation reaction was performed to insert sart 1 into the $\mathrm{pCS} 2^{+}$vector. After molecular cloning of $\mathrm{pCS}^{+}$: sart1, DNA was purified using the DNA Miniprep Kit (Qiagen). Purified DNA was linearized using Not 1 (New England BioLabs) and incubated at $37^{\circ}$ for $2 \mathrm{~h}$. RNase A was added to the reaction and incubated at $37^{\circ}$ for 5 $\min$.

Following the protocols as outlined in the mMessage machine kit (Ambion), transcription-quality plasmid DNA was prepared and RNA was synthesized from the linearized DNA plasmid. The RNA quality was analyzed on a $1 \%$ agarose gel and the quantity was measured using a ND-1000 NanoDrop Spectrophotometer. For the rescue, approximately $150 \mathrm{pg}$ of sart $1 \mathrm{mRNA}$ was injected into single-cell embryos produced from sart1 heterozygous parents. Embryos were observed daily to look for rescue of the mutant phenotype. A scoring system based on the phenotype at $4 \mathrm{dpf}$ was used to determine the rescue efficiency. Larvae were scored as wild-type, mutant, or abnormal. Embryos were considered abnormal if there was an observable defect that did not resemble a wild-type or mutant phenotype. After scoring the larvae based on phenotype, samples were also collected and genotyped using PCR and Sanger DNA sequencing to determine how many mutant larvae were rescued. The percentage rescued was based on the number of mutants (based on genotype) that had wild-type phenotypes. Abnormal 


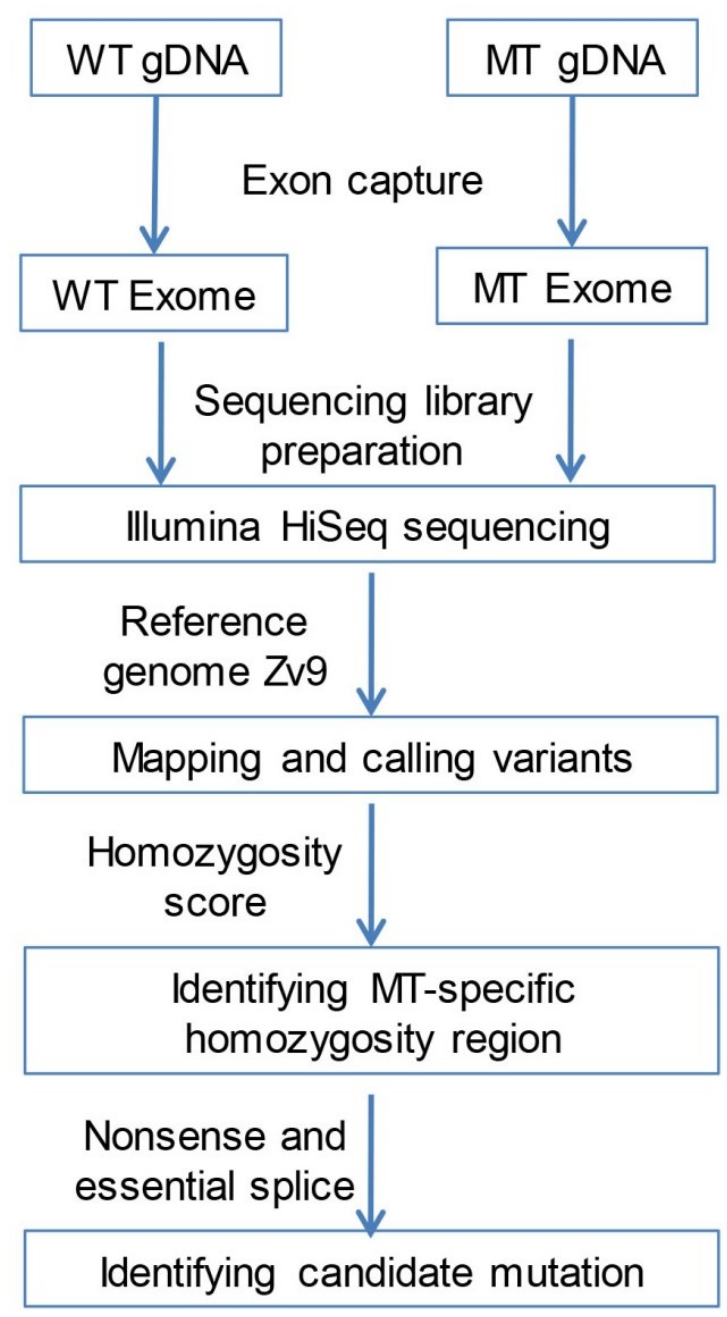

Figure 4-1. Schematic of WES Sequencing Workflow and Analysis. 
phenotypes for mutants were considered a partial rescue since most "abnormalities" were minor compared to phenotypes of uninjected mutants, such as reduced head size or slight heart edema.

\section{Results}

\section{Positional Cloning and Whole-Exome Sequencing of $c$ p27.5 Identifies Mutation in sart1}

To identify the mutated gene for $c p 27.5$, we performed genetic mapping using 192 polymorphic markers distributed evenly across the genome. For $c p 27.5$, we identified markers z2363 and z15891 by bulked segregant analysis to map the mutation to chromosome 21. Within this critical interval, we identified the marker z9233 and found no recombinants out of 48 meioses. We then tested additional polymorphic markers and counted the number of recombinants on either side of z9233 to refine the critical interval. By testing polymorphic markers, BX530031.5 and BX530023.5, selected from the zebrafish genome as published in Ensembl Zv9 (Flicek et al., 2014), we narrowed the region to $\sim 0.4 \mathrm{Mb}$. In total, we analyzed 450 meioses. A map of the defined critical interval is represented in Figure 4-2. Genes within this region include: sart1 (NM_001002673.1), rinla (XP_697152.1), slc29a2 (NM_001012501.2), and dpp3 (NM_001002683.1).

After defining the critical interval using positional cloning, we utilized WES technology to identify the gene and type of mutation. We performed WES on cp27.5 mutants and their wild-type siblings. Using this technique, we isolated a point mutation in sartl containing a $\mathrm{G}$ to $\mathrm{A}$ transition at the donor site of exon 12 . Upon sequencing wild-type and mutant cDNA, we discovered the retention of intron 12 that contained a TAA premature stop codon (Figure 4-3).

\section{sart1 mRNA Rescues Mutant Phenotype}

To verify sart 1 as the causal gene resulting in the phenotypes seen in $c p 27.5$ mutants, a rescue experiment was done by injecting sart 1 mRNA into single-celled embryos and observing them at $4 \mathrm{dpf}$ to look for rescue. We screened 462 uninjected and 375 sart 1 mRNA injected larvae. While overexpression of sart 1 mRNA in wild-type embryos did not cause any overt phenotype, sart 1 mRNA was able to rescue defects seen in mutants such as abnormal CP development, heart edema, curved body, and survived past 4 dpf. Some larvae survived as late as 8 dpf. From this rescue, we quantified the results and found that, based strictly on phenotype, the number of mutants for uninjected controls was $23.3 \%$, while mutant larvae from a spawn injected with sart 1 mRNA was $1.5 \%$ (Table 4-1). 


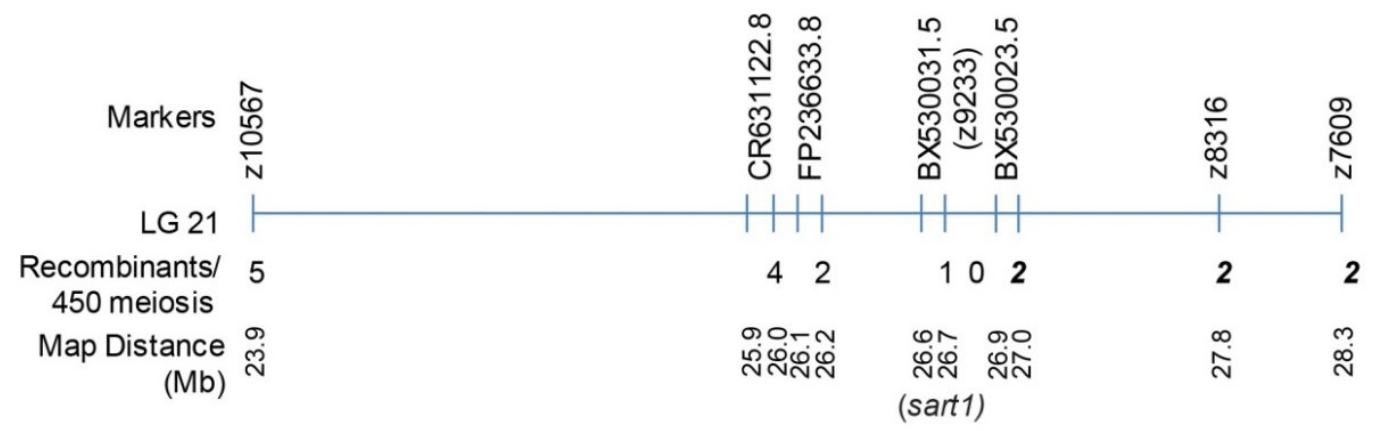

Figure 4-2. Positional Cloning Defines Critical Interval on Chromosome 21.

A critical interval was defined on Chromosome 21 using positional cloning. For bulked segregant analysis, the polymorphic marker z9233 demonstrated linkage having zero recombinants identified out of 450 meiotic events. Additional markers were analyzed both proximal and distal to z9233 to define a critical interval of approximately $0.4 \mathrm{Mb}$. The numbers of recombinants on the proximal side are shown in regular type and those distal to z9233 are shown in bold italic font. sart1 lies within the contig sequence BX530031.5. 


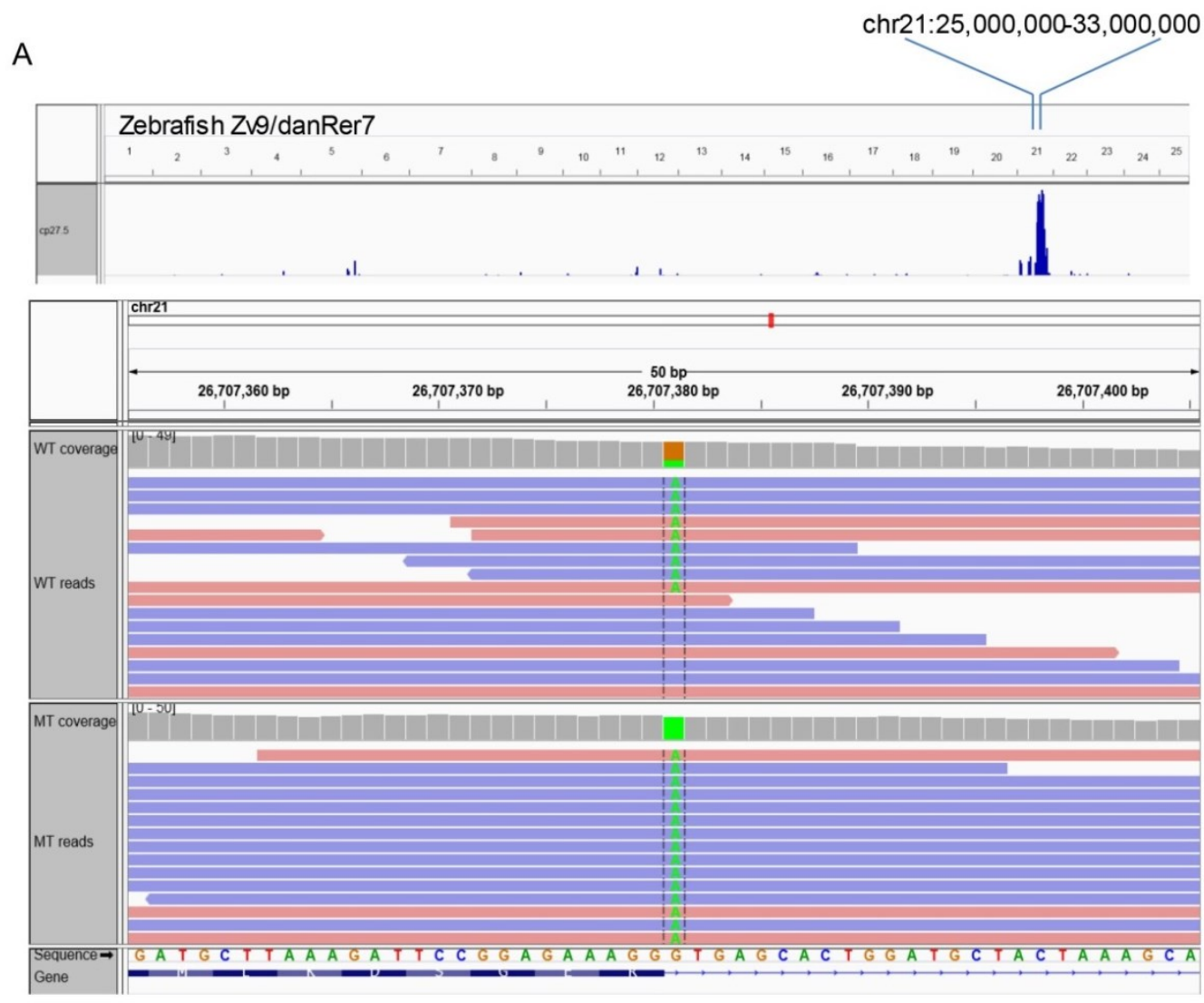

B

C

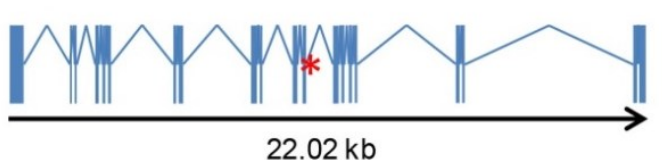

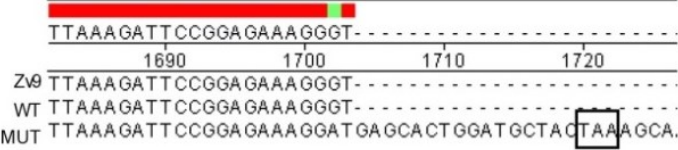

\section{Figure 4-3. WES Identifies a Point Mutation in sart1.}

In (A), the blue peak is representative of the highest homozygosity score where mutants are homozygous within this defined loci along Chromosome 21. This finding is further demonstrated in the panel below where the reference genome contains a guanine $(G)$ (in orange) at this position and all mutants have an adenine (A) (in green). An adenine is present in a portion of the wild-type sequence due to the fact that wild-type siblings may be heterozygous for the mutation. (B) and (C) confirm that the gene containing the mutation is sartl as shown by a point mutation at the end of exon 12 (red asterisk) in (B). (C) DNA Sanger Sequencing of cp27.5 wild-type and mutant cDNA confirms the G $\rightarrow$ A transition and reveals the retention of intron 12 and introduction of a premature stop codon (TAA). 
Table 4-1. $\quad$ sart1 mRNA Rescues Mutant Phenotypes.

\begin{tabular}{lcc}
\hline Samples & Total Number Screened & $\begin{array}{c}\text { Morphologically Mutant } \\
\text { (\%) }\end{array}$ \\
\hline No Injection Control & 462 & $23.3 \%$ \\
sart1 mRNA & 375 & $1.5 \%$ \\
\hline
\end{tabular}

To confirm that $c p 27.5$ mutants were among those rescued, we collected uninjected cp27.5 wild-type and mutant larvae and sart 1 mRNA injected larvae and sequenced individual DNA. We sequenced a total of 90 larvae with $20 \%$ of those having a mutant genotype. Not surprisingly, we identified mutants with wild-type phenotypes as represented in Figure 4-4. Some mutants appear to have complete rescue as shown in the lower right-hand panel, while others had slight heart edema, smaller head size and somewhat larger CP compared to wild-type (presented in two upper panels under Rescue in Figure 4-4). Sequencing results are quantified in Figure 4-5. Based on the sequencing data, we identified $10 \%$ of mutants we scored as having a wild-type phenotype. The other $10 \%$ were scored as abnormal or having only a partial rescue. However, even partially rescued mutants are far more phenotypically normal compared to uninjected mutants shown in the middle panel of Figure 4-4.

\section{Discussion}

A recent study has cited that more than 9,000 mutants have been discovered as a result of forward genetic screens in zebrafish, but many of the mutants have not been further studied and $62 \%$ of them are uncloned (Obholzer et al., 2012). This is because of the limitations involved in positional cloning including time, money, and effort it takes to identify the gene (Obholzer et al., 2012). Hundreds of PCRs and sequencing of multiple candidate genes is sometimes necessary to identify the mutation. Other times, the gene is never identified simply because of its location along the chromosome, such as near the telomere where multiple recombination events can occur. New technology with next generation sequencing is redefining the way genes and their mutations are discovered. WES has been used for many studies in humans and animal model systems. In human samples, sequences can be compared between parents and their children to isolate de novo mutations, between unrelated persons to identify new variations of the same gene, or a sequenced region within the genome can be compared between multiple persons within the same family to look for variations of a particular gene (Bamshad et al., 2011). These studies have identified genes related to a number of human conditions including hearing loss, cardiovascular disease, and cancer. An extensive list of the mutations related to these conditions is presented by Rabbani et al. (Rabbani et al., 2014).

In a recent study, WES was used in zebrafish to identify the gene mutation for four mutants all of which were characterized by having kidney cysts. Using this approach, they were able to identify the genes and types of mutations, including nonsense, splice site, and missense mutations for all four mutants (Ryan et al., 2013). A 

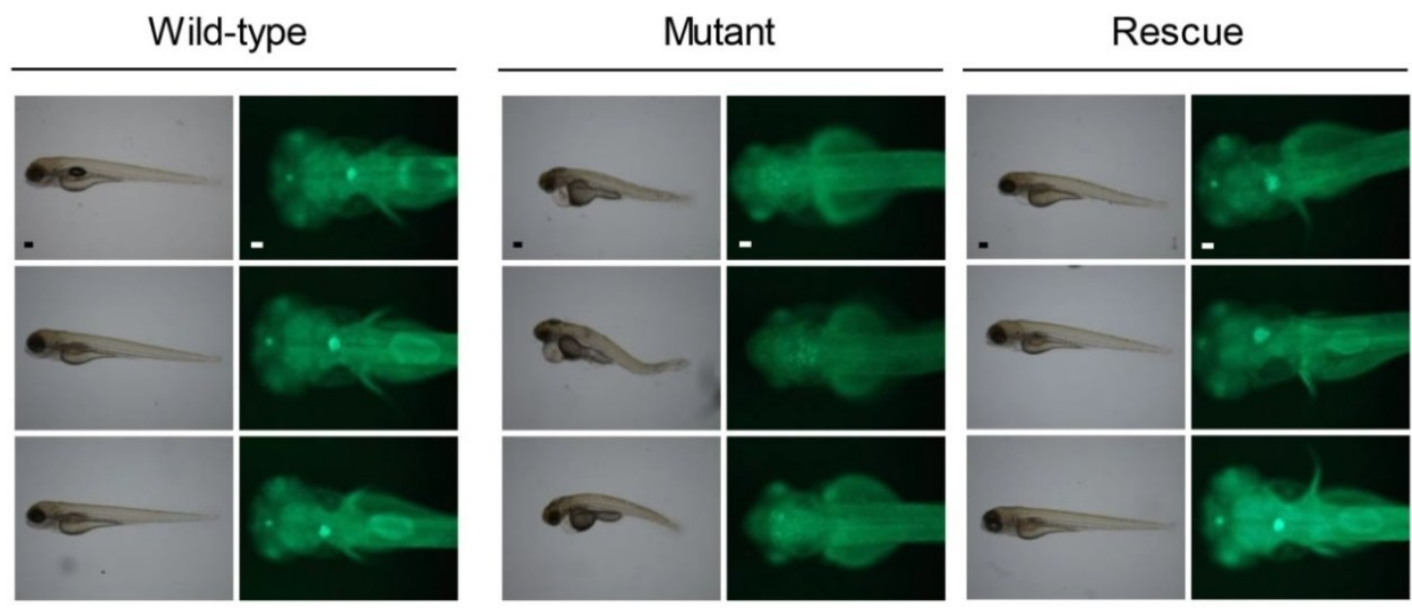

Figure 4-4. sart1 Rescues Mutant Phenotypes.

Dorsal bright field and fluorescent images are shown for three uninjected wild-type, three uninjected mutant, and three sartl injected mutant larvae in the left, middle, and right panels, respectively. Injection of sart $1 \mathrm{mRNA}$ resulted in mutants with phenotypes similar to wild-type larvae. The three rescued mutants represent a range of phenotypes we observed after sart $1 \mathrm{mRNA}$ injection. Scale bars are $100 \mu \mathrm{m}$ for bright field images at $3 \times$ magnifications and $50 \mu \mathrm{m}$ for fluorescent images acquired at $9 \times$ magnifications. 


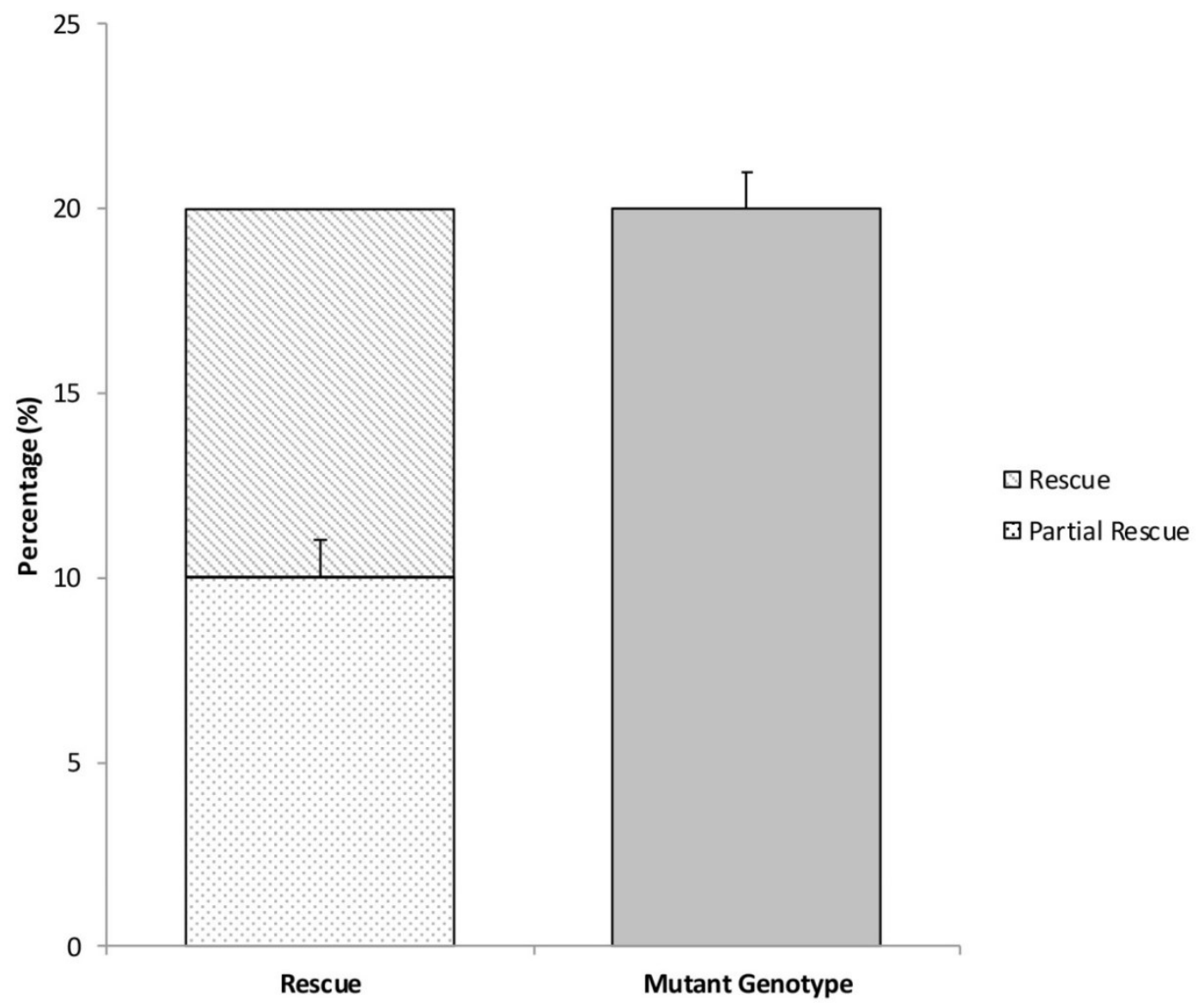

Figure 4-5. Quantification of sart1 Rescue.

Sequencing a total of 90 cp 27.5 wild-type and mutant larvae identified $20 \%$ with a mutant genotype. Of the total number of mutants $10 \%$ were scored as wild-type and $10 \%$ were scored as abnormal based on phenotype. The $10 \%$ abnormal were considered to be partially rescued. Error bars are based on the mean of 3 rounds of injections using standard error. 
unique study by Kettleborough et al. identified protein coding genes in zebrafish by performing WES on the $\mathrm{F}_{1}$ generation and isolating nonsense and splice site mutations (Kettleborough et al., 2013).

WES offers an advantage over WGS in that it can provide better coverage of the genome. Coverage is described as the number of times the genome is sequenced and can be calculated as follows in Equation 4-1:

$$
\mathrm{C}=\mathrm{LN} / \mathrm{G}
$$

Where $\mathrm{C}$ is coverage, $\mathrm{G}$ is the haploid genome length, $\mathrm{L}$ is the read length, and $\mathrm{N}$ is the number of reads. This equation is also known as the Lander/Waterman equation (Lander and Waterman, 1988). Although WGS usually has 4x coverage or less, we had 20x coverage or greater for our samples using WES. While WES is improving, there are still setbacks that can occur with this strategy. For example, a gene may be undetected if it is novel and unrecognized by the exome capture, there may be poor coverage of that region, or incorrect variants might be recognized if the reads become incorrectly mapped or unaligned (Bamshad et al., 2011).

In our current study, we found that WES provided a significant advantage in regards to the reduced time required to identify the gene and the type of mutation. By first defining the critical interval using positional cloning, we were able to validate WES as a suitable method for identifying genes in zebrafish since both strategies isolated the same genomic region. However, had we not performed WES, traditional mapping strategies would have required additional time and effort sequencing multiple genes within the critical interval. Studies in mice have also shown that by using course genetic mapping to identify the chromosome location followed by WES significantly reduces the "validation burden" and helps to eliminate more false positives from the data (Fairfield et al., 2011).

For our study, we decided to sequence both mutant and wild-type siblings and align the sequences to the Ensembl ZV9 reference genome (Flicek et al., 2014) rather than compare mutants to the reference genome alone. While this approach is more costly by having two samples, we were able to more efficiently circumvent any nucleotide changes that may simply be polymorphic to the strain. In addition, the DNA used for sequencing was from larvae whose parents had been outcrossed into a polymorphic strain. This step creates greater diversity throughout the genome except at the locus containing the mutation, since the phenotype caused by the gene was specifically selected for after outcrossing. In scoring for homozygous regions, some wild-type samples also contained an adenine as seen in the mutants. This is because the wild-type sibling DNA also contained heterozygous carriers for the mutation. Comparing homozygous wild-type and homozygous mutant siblings may provide a more obvious map position of the mutation.

Additional validation of sartl as the target gene was done by performing mRNA rescue experiments. Injections of mRNA into single-cell embryos prevented forebrain 
necrosis at $2 \mathrm{dpf}$ which is the earliest detectable phenotype in cp27.5 mutants. Although mRNA rescue is transient and is not sustained far past $4 \mathrm{dpf}$ in zebrafish, we were able to rescue phenotypes that begin at 3-4 dpf such as heart edema, small head and eyes, reduced heartbeat and circulation. Mutants also had a longer lifespan than their uninjected siblings. Important to this study, we were also able to restore normal CP development. From this analysis, a number of wild-type larvae were scored as abnormal. Abnormal phenotypes for wild-type larvae may be due to off target effects of the injection. Also, when sequencing wild-type and mutant DNA from sart 1 mRNA injections, we identified only $20 \%$ as mutant rather than the $25 \%$ predicted by Mendelian genetics. We attributed the decreased percentage due to damaged embryos from microinjection that may not have survived past early development. For uninjected samples, we observed between $23-25 \%$ mutant. Since this gene was identified from a genetic screen for the $\mathrm{CP}$, future studies will determine whether sart 1 is directly affecting $\mathrm{CP}$ formation and investigate potential pathways sartl may be activating or suppressing that contribute to $\mathrm{CP}$ development and function. Because sartl is involved in formation of the spliceosome that converts precursor mRNA into mature mRNA, defects in the splicing process may produce altered transcripts that result in defective translation and formation of proteins necessary for $\mathrm{CP}$ formation. The lack of coalescence in $\mathrm{CPe}$ may be due to alternative splicing in transcripts encoding for tight junction or adherens proteins or signals involved in cell survival and migration.

\section{Conclusion}

Supplemental to previous publications, our current study endorses the use of WES in zebrafish by identifying the gene sartlprecisely within the critical interval we defined previously by positional cloning. We confirmed WES data using Sanger DNA sequencing to sequence wild-type and mutant cDNA. In doing so, we identified a retained intron and the introduction of a premature stop codon in mutant samples. Using mRNA rescue, we identified $10 \%$ of mutants having wild-type properties with another $10 \%$ partially rescued. These experiments further validated that sart 1 is the gene responsible for the mutant phenotype. Future studies will characterize sart 1 mutants to define its role in CNS development and WES will be used to map the gene for additional mutants from the genetic screen described in Chapter 3. 


\section{CHAPTER 5. CHARACTERIZATION OF SART1 MUTANT REVEALS UP-REGULATION OF GENES INVOLVED IN APOPTOSIS AND DOWN-REGULATION OF VISION-RELATED GENES}

\section{Introduction}

Sart1 also known as U4/U6.U5 tri-small nuclear ribonucleoprotein-associated protein 1, HAF in mouse, and Snu66 in yeast, is a complex protein involved in U4/U6.U5 tri-snRNP recruitment to the spliceosome (Makarova et al., 2001). Sart1 has two protein forms. One is an 800 amino acid sequence located in the nucleus of proliferating cells and contains a leucine zipper motif suggesting it may bind to DNA (Shichijo et al., 1998). Another 259 amino acid protein is synthesized by a -1 frame shift in an internal ribosomal entry site that has been shown to be expressed in the cytosol in squamous cell carcinomas (Shichijo et al., 1998).

In addition to splicing, Sart1 has been implicated in a number of different processes such as cell cycle arrest and apoptosis. It has been suggested as a target for gene therapy due to its activation of apoptosis and its role as an antigen recognized by cytotoxic T lymphocytes (CTLs) in certain types of cancer (Hosokawa et al., 2005). More recently, Sart1 has been shown to contribute to drug resistance in cancer cells. Silencing of this gene resulted in increased apoptosis in colorectal cancer cells in response to 5-Fluorouracil and SN38 drug treatment (Allen et al., 2012). Similarly, Sart1 has been shown to aid in Mcll activity, a pro-survival factor and contributor to drug resistance in cancer (Laetsch et al., 2014). Knockdown of Sart 1 caused Mcl1-dependent neuroblastoma cells to become more responsive to treatment (Laetsch et al., 2014).

While splicing genes are thought to be ubiquitously expressed, recent evidence suggests that certain components of the spliceosome may be temporally or spatially regulated. Studies have identified spliceosome components such as U2 that have high RNA levels specifically in the cerebellum and mutations in this gene result in neurodegeneration (Jia et al., 2012). In zebrafish, we observed that sartl is expressed specifically in the brain and is actually up-regulated in sart 1 mutants providing further evidence that splicing components may be regulated or expressed in specific tissues and may result in tissue-specific phenotypes.

Using RNA-Seq analysis, we identified that sart 1 itself is up-regulated due to the mutation described previously in Chapter 4. It also results in up-regulation of genes essential to the apoptotic pathway such as tp53 and $m d m 2$. Conversely, this mutation causes genes regulating eye development and function to be down-regulated. This analysis confirmed our observations of increased cell death observed in the brain and eyes and lack of retinal lamination in these mutants. We also observed an up-regulation of other components of the spliceosome such as U6 SnRNA-Associated Sm-Like Protein (lsm 7$)$ and pre-mRNA processing factor 31 (prpf31), which may indicate a compensation mechanism due to defects in sartl function. 
While previous studies have identified the major role of Sart 1 as recruiting the U4/U5/U6 tri-snRNP to the spliceosome, understanding how Sart 1 regulates the expression of other genes will reveal how spliceosome components not only play a role in splicing, but also other cellular processes such as apoptosis and the cell cycle and conditions such as degeneration and cancer.

\section{Materials and Methods}

\section{Reverse Transcriptase-PCR (RT-PCR)}

Larvae were collected on ice in $50 \mu \mathrm{L}$ Trizol (Life Technologies) and stored at $-80^{\circ}$ until use. Samples were homogenized with a dounce for $30 \mathrm{~s}$. Additional Trizol was added to bring the final volume to $100 \mu \mathrm{L}$. Samples were mixed and incubated at RT for $5 \mathrm{~min}$. Chloroform was added $(0.2 \mathrm{~mL}$ for every $1 \mathrm{~mL}$ of Trizol) and samples were mixed and incubated at RT for $5 \mathrm{~min}$. Samples were centrifuged at $4^{\circ}$ at $12,000 \mathrm{x} g$ for $15 \mathrm{~min}$. The top aqueous layer was transferred to a new tube. Isopropanol $(0.5 \mathrm{~mL}$ for every $1 \mathrm{~mL}$ of original volume of Trizol) and $2 \mu \mathrm{L}$ of glycogen were added to the clear aqueous solution followed by mixing and incubating a RT for 5 min. Samples were centrifuged at $4^{\circ}$ at $12,000 \mathrm{x} g$ for $10 \mathrm{~min}$. Isopropanol and chloroform were removed leaving a RNA pellet. The pellet was washed in $100 \mu \mathrm{L} \mathrm{75 \%} \mathrm{ethanol.} \mathrm{Wash} \mathrm{volumes}$ were the same as the original Trizol volume. Samples were centrifuged at 4 at 7,500 $\mathrm{g}$ for $5 \mathrm{~min}$. Ethanol was removed and the pellet was air-dried for $20 \mathrm{~min}$ or until excess ethanol evaporated. RNA was resuspended in $20 \mu \mathrm{L}$ nuclease-free water.

cDNA synthesis was performed using SuperScript ${ }^{\circledR}$ III Reverse Transcriptase (Life Technologies). A mixture of $50 \mu \mathrm{M}$ oligo dT, $10 \mathrm{mM}$ dNTP, and $8 \mu \mathrm{L}$ of RNA product was incubated at $65^{\circ}$ for $5 \mathrm{~min}$ and placed on ice for $>1 \mathrm{~min}$. A mixture of $10 \mathrm{x}$ RT buffer, $25 \mathrm{mM} \mathrm{MgCl}_{2}, 0.1 \mathrm{M}$ DTT, RNAse Out, and Superscript III was added to the mix containing the RNA. The reaction was incubated at $50^{\circ}$ for at least $50 \mathrm{~min}$. The reaction was terminated by incubating at $85^{\circ}$ for $5 \mathrm{~min}$. Samples were chilled on ice and $1 \mu \mathrm{L}$ of RNAse $\mathrm{H}$ was added to each reaction. Samples were incubated at $37^{\circ}$ for $20 \mathrm{~min}$ and then stored at $-20^{\circ}$. cDNA was diluted 1:5 in water for PCR. RT-PCR was done using the AccuPrime Taq DNA Polymerase System (Invitrogen). Primer sequences included sart1F1:5'-CGTTTTTAAGCCAAAGTCTGTGCTG-3' and sart1R1:

5'-CACCTCCTTCTTTCTCGTCATCCTT-3'. Samples were analyzed on a 1\% agarose gel.

\section{Whole Mount In Situ Hybridization}

The RNA probe was synthesized from the PCRII TOPO vector containing the 2.5 kb sart1 fragment as mentioned above using the DIG RNA Labeling Kit (SP6/T7) (Roche). A probe for deltaC was also synthesized to use as a control. The probes were purified using illustra Probe Quant G-50 micro columns (GE Healthcare) and 
resuspended in $80 \mu \mathrm{L}$ hybridization buffer. Hybridization buffer was prepared as described in Thisse and Thisse (Thisse and Thisse, 2008). Probes were analyzed on a 1\% agarose gel to determine the quality of the RNA.

cp27.5 wild-type and mutant larvae were collected at 3dpf were anesthetized in $0.02 \%$ tricaine and fixed in 4\% paraformaldehyde (PFA) (Electron Microscopy Sciences) made in 1x Dulbecco's PBS (Sigma, cat. no. D-5652) and Diethyl pyrocarbonate (DEPC) (Sigma Aldrich) -treated water. Larvae were stored at $4^{\circ}$ for $16 \mathrm{~h}$. Samples were then washed in 100\% methanol (Sigma Aldrich) at room temperature for 15 min and stored in fresh methanol at $-20^{\circ}$ until use. The following procedure was carried out in $1.5 \mathrm{~mL}$ Eppendorf tubes: Samples were rehydrated to 1x PBT (phosphate buffered saline/ $0.1 \%$ tween 20 (Sigma) as described previously by Thisse and Thisse (Thisse and Thisse, 2008). Larvae were digested with $10 \mu \mathrm{g} / \mathrm{mL}$ Proteinase K (New England Biolabs) at room temperature for $30 \mathrm{~min}$ followed by treatment with 4\% PFA for 20 min at room temperature. After washing 4 times for $5 \mathrm{~min}$ in $1 \mathrm{x}$ PBT, larvae were prehybridized for 2 $\mathrm{h}$ at $67^{\circ}$ in $700 \mu \mathrm{L}$ hybridization buffer. Larvae were then incubated in $200 \mu \mathrm{L}$ of hybridization buffer containing probe at $67^{\circ}$ for approximately $48 \mathrm{~h}$. Probes were used at a 1:100 dilution. After incubation, larvae were washed following the series described in Thisse and Thisse (Thisse and Thisse, 2008) except for the hybridization buffer/ 2x SSC series was done for $15 \mathrm{~min}$ each and the $0.2 \mathrm{x} \mathrm{SSC} / \mathrm{PBT}$ series was done for $5 \mathrm{~min}$ each. Larvae were blocked in 2\% lamb serum (Gibco) made in PBT at room temperature for 1 h. After blocking, larvae were incubated in anti-DIG antibody (Roche) at 1:5000 made in blocking buffer at $4^{\circ}$ overnight. Washes were carried out the next day as described in Thisse and Thisse (Thisse and Thisse, 2008).

To develop, $500 \mu \mathrm{L}$ alkaline phosphatase buffer containing 5-Bromo-4-chloro-3-indolyl phosphate/nitro-blue tetrazolium (BCIP/NBT) (Sigma-Aldrich) was added to the larvae in 12 well plates. Larvae were incubated in the dark and observed periodically until a purple precipitate appeared. The reaction was terminated by removing staining solution and adding 1x PBS/1 mM EDTA followed by washes in $1 \times$ PBS. Samples were stored in $>99 \%$ glycerol (Sigma-Aldrich) and imaged on a Nikon SMZ1500 epifluorescence stereomicroscope with Nikon NIS-Elements 3.1 software. All reagents were prepared following the protocol as described in Thisse and Thisse (Thisse and Thisse, 2008).

\section{Immunohistochemistry (IHC)}

IHC was performed as described in on pg. 30. Primary antibodies included rabbit anti-activated Caspase 3 (1:200; Cell Signaling), rabbit anti-Glut1 (1:200; Novus), mouse anti-SV2 (1:300; Developmental Studies Hybridoma Bank), mouse anti-Zpr1 (1:100; ZIRC), mouse anti-HuC/HuD neuronal protein (1:500; Life Technologies), and rabbit anti-S100ß (1:1000; Dako). Secondary antibodies included Alexa Fluor goat anti-rabbit 488 (1:200; Invitrogen) and Alexa Fluor goat anti-mouse 555 (1:200; Invitrogen). Antibody dilutions were prepared in blocking buffer. Sections were counterstained with $1 \mu \mathrm{g} / \mathrm{mL}$ DAPI (Roche) for $1 \mathrm{~min}$, rinsed briefly in PBS, and mounted with Fluoromount 
(Electron Microscopy Sciences). Images were taken on a Nikon C1Si laser scanning confocal microscope and analyzed using NIS-Elements AR 3.0 software.

\section{Whole Mount IHC}

Whole mount IHC was performed as described on pg. 30. Antibodies used included rabbit anti-GFP (1:100; Invitrogen), rabbit anti-activated Caspase-3 (1:100; Cell Signaling), mouse anti-Zpr1 (1:50; ZIRC), and mouse anti-Cldn5 (1:100; Invitrogen), Alexa Fluor goat anti-rabbit 488 (1:200; Invitrogen) and Alexa Fluor goat anti-mouse 555 (1:200; Invitrogen). Samples were imaged on Nikon C1Si laser scanning confocal microscope. Z-stacks were compiled to create maximum intensity projection images using Nikon NIS-Elements 3.0 imaging software.

\section{Western Blot}

An equal number of wild-type and mutant larvae were each collected in a $1.5 \mathrm{~mL}$ Eppendorf tube, the embryo media removed, and stored at $-80^{\circ} \mathrm{C}$. Homogenization buffer (2x) (Mini Complete tablet, Roche) containing 0.002\% DNase (Ambion) was added and larvae were homogenized on ice. Samples were removed from ice and SDS/gel loading buffer containing DTT was added to the homogenate. Samples were then placed in boiling water for $5 \mathrm{~min}$, cooled afterwards at RT, and centrifuged briefly before gel loading. Samples and the Precision Plus Kaleidoscope Protein Standard ladder (BioRad) were run on a $4-15 \%$ polyacrylamide gel (BioRad) at $200 \mathrm{~V}$ for 30 ' at RT. Proteins were transferred to a nitrocellulose membrane using the Trans-Blot Turbo Transfer System (BioRad). Following protein transfer, the nitrocellulose membrane was blocked in 5\% milk for $1 \mathrm{hr}$. Membranes were incubated in $5 \mathrm{~mL}$ of primary antibody at $4^{\circ} \mathrm{C}$ overnight. The membrane was then washed in $1 \mathrm{x}$ Tris-buffered saline $/ 0.1 \%$ Tween (TBST) at RT and incubated in $20 \mathrm{~mL}$ of secondary antibody for $1 \mathrm{hr}$ at RT. After incubation, the membranes were washed in 1x TBST and imaged using LI-COR Odyssey 1393 and Odyssey Infrared Imaging System Version 3.0. Primary antibodies included rabbit anti-p53 (1:500; GeneTex) and mouse anti-actin (1:1000; Sigma Aldrich). Secondary antibodies included goat anti-rabbit IRDye ® 680 (1:20,000; LI-COR) and goat anti-mouse IRDye ${ }^{\circledR} 800 \mathrm{CW}(1: 15,000 ;$ LI-COR).

\section{Doxycycline Treatment}

cp27.5 wild-type and mutant larvae were treated at $24 \mathrm{hpf}$ with $50 \mu \mathrm{g} / \mathrm{mL}$ doxycycline (ClonTech). Larvae were observed at $3 \mathrm{dpf}$ to look for GFP expression in the CNS and imaged using a Nikon SMZ1500 epifluorescence stereoscope and Nikon NIS-Elements 3.1 software. 


\section{Fluorescent Tracer Injections}

Wild-type and mutant $c p 27.5$ larvae were anesthetized in $0.02 \%$ tricaine and co-injected intravenously into the common cardinal vein with approximately $1.8 \mathrm{~nL}$ of 10 $\mathrm{mg} / \mathrm{mL} 3-\mathrm{kDa}$ Cascade blue dextran, 10-kDa rhodamine dextran, and a 40-kDa anionic fluorescein dextran (Invitrogen). Injections were performed using a micromanipulator and a pneumatic picopump (World Precision Instruments). Immediately after injection, larvae were embedded in 1.2\% low-melting-point agarose (Invitrogen) made in egg water. Larvae were imaged within 30 min post-injection on a Nikon C1Si confocal microscope and analyzed using Nikon EZC1 3.91 software.

\section{RNA-Seq}

RNA was extracted as described before (see RT-PCR pg. 81) from wild-type and mutant cp27.5 larvae at $72 \mathrm{hpf}$. Samples were submitted to the Genome Sequencing Facility Hartwell Center for Bioinformatics and Biotechnology at St. Jude Children's Research Hospital for QC and quantification analysis and sequencing. Approximately $500 \mathrm{ng}$ total RNA was used to generate libraries with the TruSeq RNA v2 kit according to the manufacturer's instructions (Illumina, San Diego, CA.). PolyA-selected RNAs from wild-type and mutant zebrafish were sequenced using Illumina HiSeq 2500. The raw 100-bp pair end reads were mapped to zebrafish genome (Zv9) using STAR (Dobin et al., 2013). Duplicated reads were marked using Picard (http://picard.sourceforge.net/). The detailed mapping statistics are listed in Table 5-1. Considering genome duplication, FPKM (fragments per kilobase of exon per million fragments mapped) for each RefSeq gene was calculated as following: first fragments mapped to each exon region of a gene, regardless of their genomic location, were marked and summed up as the gene counts. The final FPKM value for the gene was calculated as shown in Equation 5-1:

$$
\mathrm{FPKM}=\mathrm{F}_{\text {gene }} * 1000 * 1000,000 /\left(\mathrm{F}_{\text {allexon }} * \mathrm{~L}_{\text {gene }}\right)
$$

Where $F_{\text {gene }}$ is the total fragments mapped to a gene, $F_{\text {allexon }}$ is total fragments mapped to exonic region in $\mathrm{Zv} 9$, and $\mathrm{L}_{\text {gene }}$ is size of unique exonic bases for the gene. Differential expression analysis was done using purely fold change between the mutant and wild type cells. GO analysis was done using DAVID (Huang, 2009a, b) with differentially expressed genes that have moderate expression level (FPKM $>0.1)$.

\section{Quantitative Real Time PCR (qRT-PCR)}

RNA was extracted from nine $c p 27.5$ wild-type and nine $c p 27.5$ mutant larvae and cDNA was synthesized using the same method as described in RT-PCR on pg. 81. RNA concentrations were normalized between wild-type and mutant prior to DNAse I treatment. Samples were treated with DNAse I (Ambion) and 10x Reaction buffer made in DEPC water at $37^{\circ}$ for 15 min followed by treatment with $25 \mathrm{mM}$ EDTA (Ambion) at 
Table 5-1. Mapping Statistics of RNA-Seq Data.

\begin{tabular}{lccccc}
\hline Sample & Reads & Mapped & NonDupMapped & Mpd\% & Dup\% \\
\hline $\begin{array}{l}c p 27.5 \\
\begin{array}{l}\text { Wild-type } \\
\text { cp27.5 }\end{array}\end{array}$ & 61515460 & 58536062 & 45999884 & 95.16 & 21.42 \\
Mutant & 99288072 & 94481824 & 74744636 & 95.16 & 20.89 \\
\hline
\end{tabular}

Mdp (mapped); Dup (duplicate).

$65^{\circ}$ for 15 min to inactivate DNAse I. Samples were then placed on ice for 1 min. After cDNA was synthesized, $20 \mathrm{ng}$ of cDNA was used per reaction for quantitative real-time PCR (qRT-PCR). Primers were designed using NCBI Primer-BLAST and prepared at 500 nmol.. qRT-PCR was performed using SYBR ${ }^{\circledR}$ Green PCR Master Mix (Life Technologies) on a 7500 Fast Real Time PCR machine (Applied Biosystems) and analyzed using 7500 Software v2.0.6. Relative gene expression was calculated using the Delta Delta CT (ddCt) method relative to the reference gene $\beta$-actin. A bar graph showing the normalized fold change in mutant compared to wild-type was made in GraphPad Prism. Error bars are based on the mean of 3 rounds of injections using standard error. The primer templates used for each gene are found in Table B-1.

\section{Results}

\section{sart1 mRNA Is Maternally Derived and Expressed in the Zebrafish Brain}

To determine when sart1 is expressed, we performed RT-PCR using cDNA from day 0 to day 5 wild-type samples. We observed sartl expression for each time point and also established that sart 1 is maternally derived based on its expression at day 0 (Figure 5-1A). Because we observed phenotypes primarily within the head and eye region in cp27.5 mutants, we were curious to determine whether sartl is spatially or temporally regulated within these regions or ubiquitously expressed. We looked at three developmental time points to determine sartl expression: $48 \mathrm{hpf}$ when we begin to observe mutant phenotypes, $72 \mathrm{hpf}$, and $96 \mathrm{hpf}$ when mutants begin to deteriorate. Using whole-mount in situ hybridization, we observed sartl localized in the head at $48 \mathrm{hpf}$ (Figure 5-1B). This expression was consistent throughout development although it appeared to become less prominent in this region by $96 \mathrm{hpf}$ (Figure 5-1B). We also wanted to see whether there were differences in expression between $c p 27.5$ wild-type and mutant. While sartl was seen in the head at $72 \mathrm{hpf}$ for both wild-type and mutant, sartl was more highly expressed in mutant compared to wild-type (Figure 5-1C). We do not contribute increased expression to increased permeability of the probe in mutant due to the fact that the positive control, deltaC, was not intensified in mutants (Figure 5-2). Interestingly, deltaC expression which is normally localized in the brain and eyes was disordered and reduced in $c p 27.5$ mutants. 
A

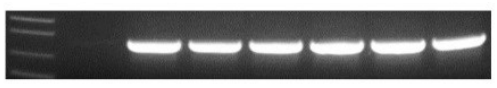

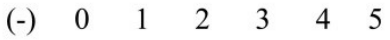

B

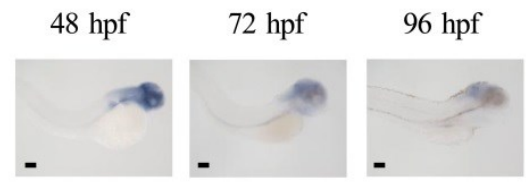

C

sartl antisense sartl sense

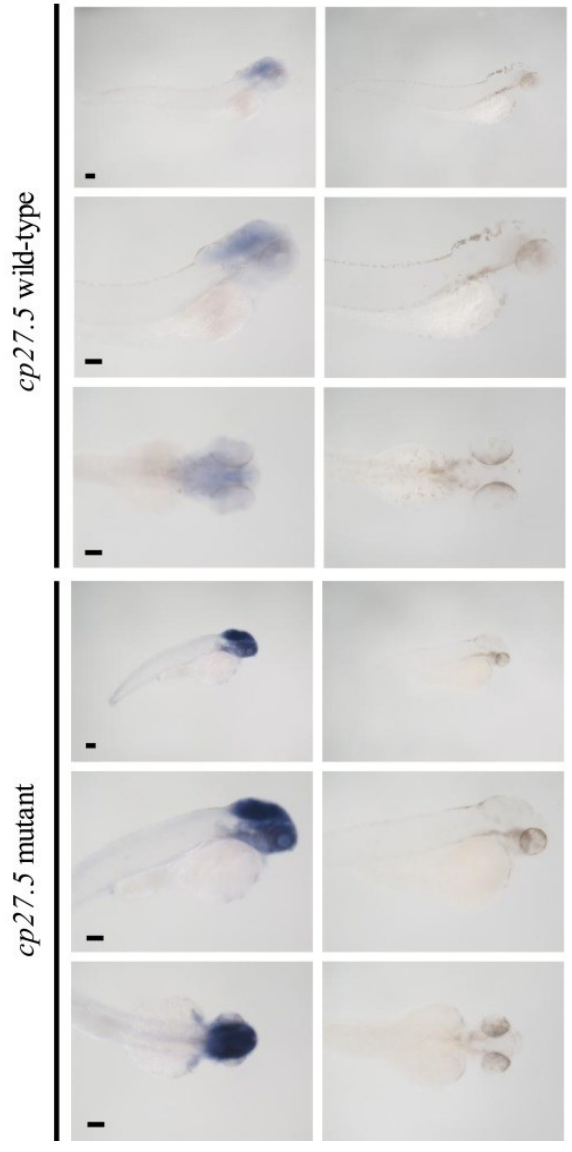

Figure 5-1. RT-PCR and Whole Mount In Situ Hybridization Identifies sart1 Expression.

(A) RT-PCR shows sartl expression from day 0 to day 5 in zebrafish larvae including a water negative control (-). (B) Whole mount in situ hybridization shows sart1 expression localized to the brain at $48 \mathrm{hpf}, 72 \mathrm{hpf}$, and $96 \mathrm{hpf}$. (C) Whole mount in situ hybridization shows localization and relative expression levels of sart 1 incp27.5 wild-type and mutant larvae at $72 \mathrm{hpf}$. Images are ordered as lateral view at low magnification, lateral view at high magnification, and dorsal view at high magnification for both antisense (left column) and sense (right column). Scale bars are $50 \mu \mathrm{m}$. 


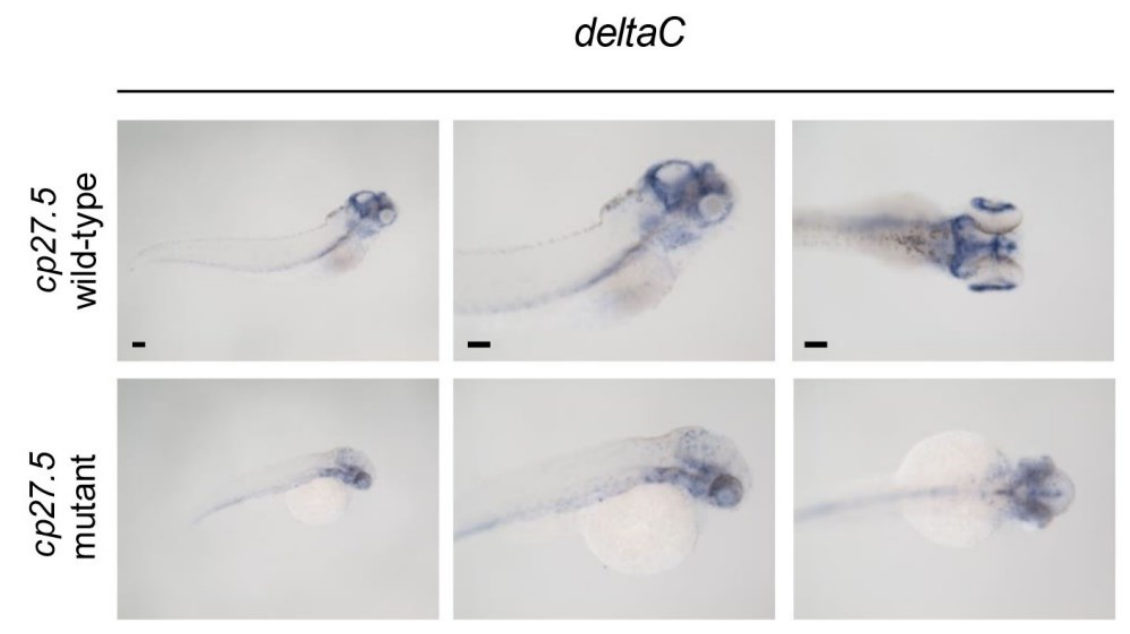

Figure 5-2. deltaC Expression Is Disordered in cp27.5 Mutants.

Whole mount in situ hybridization using deltaC as a control probe identified disordered expression in cp27.5 mutants (bottom panel) compared to wild-type (top panel). In wild-type, deltaC is localized to the retina proliferative zone and brain ventricular zone. deltaC intensity is decreased in cp27.5 mutant. While the probe does label the ventricular zone and eyes, the patterning is altered in mutant. Scale bars are $50 \mu \mathrm{m}$. 
sart1 Mutants Have Altered Protein Expression of Brain and Eye Markers Along with Increased Activated Caspase 3

Because sart1 expression was localized to the brain we wanted to determine if there were other proteins in this region whose expression may be affected by sart 1 up-regulation. Using IHC, we looked at Zpr1, a marker that labels the pineal gland in the brain and photoreceptors in the eye. cp27.5 mutants had little to no Zpr1 expression in the eye (Figure 5-3A and 5-3B). Counterstaining with DAPI revealed that retinal lamination was also absent. However, Zpr1 expression was present in the pineal gland in mutants indicating that not all Zpr1 expression was affected by sart1 (Figure 5-4). Another protein, SV2, that labels synaptic vesicles in the brain and eye, was also affected. Not surprisingly, mutants had no SV2 expression in the eye (Figure 5-3C and 5-3D). However, SV2 was present in the brain and appeared to be slightly increased compared to wild-type.

Because we did not detect expression of Zpr1 and SV2 in the eye and because mutations in splicing factors have previously been shown to contribute to retinal degeneration, (Chen et al., 2014; Yin et al., 2011) we hypothesized that cells in the eye may be undergoing apoptosis. To test this hypothesis, we used an activated Caspase-3 antibody. While no activated Caspase-3 was detected in cp27.5 wild-type larvae (Figure 5-3E), it was significantly expressed throughout the eye of cp27.5 mutants (Figure 5-3F). Caspase-3 was also detected in the dorsal region of the brain near the ventricle (Figure 5-3F inset and Figure 5-5). To determine if vasculature was affected, we used Glut1, to visualize blood vessels in the brain and eye. While mutants do have blood vessel formation, they are not as numerous and extensive as those seen in wild-type (Figure 5-3G and 5-3H). We also examined neuronal markers such as $\mathrm{HuC}$ labeling neurons and $\mathrm{S} 100 \beta$ detecting astrocytes. HuC could be detected in mutant brain, but little if any was present in the eye (Figure 5-3I and 5-3J). Because the original line from cp27.5 was generated using the Tet-On system containing a HuC promoter driving GFP expression, we confirmed these results by treating wild-type and mutant larvae with doxycycline to induce neuron-specific GFP expression. As seen with the HuC antibody, the brain and spinal cord contain $\mathrm{HuC}$ expression in wild-type and mutant, but there is little to no expression in the eye for mutants (Figure 5-6). For S100 $\beta$, we detected expression in the brain and eye of wild-type larvae (Figure 5-3K), but detected little if any expression in mutant (Figure 5-3). Overall, these findings suggested that sart1 is affecting expression of genes specifically expressed in the eye and occasionally in the brain, ultimately resulting in cell death.

\section{sart1 Mutants Have Increased Permeability in the Brain Ventricle}

Although there appeared to be a reduced number of blood vessels in the brain, we wanted to examine whether reduced vasculature led to increased permeability throughout the larva. We injected fluorescent tracers of different molecular weights intravenously into wild-type and mutant at $2 \mathrm{dpf}$ and looked for leakage. Because the increased permeability may be size-selective, we co-injected a 3-kDa cascade blue, a 10-kDa 
Figure 5-3. IHC Characterizes cp27.5 Mutants.

IHC identifies additional abnormalities in protein expression in $c p 27.5$ mutants at $3 \mathrm{dpf}$. All sections are counterstained with DAPI (blue). Zpr1 (red) labels photoreceptors in the eye which are present in wildtype (A) but not in mutant (B). SV2 labels synaptic vesicles within the brain and as seen within the inner plexiform layer of the eye in wild-type (C). SV2 is present in the brain, but absent in the eye in mutant (D). Caspase 3 is not expressed in wild-type (E), but is prominent in the eyes in mutant (F). Mutant brain also contains punctate Caspase 3 expression (inset). Glut 1 is noticeably expressed in brain and eye vasculature in wild-type $(\mathrm{G})$, but mutants have faint Glut1 expression in brain vasculature (arrows) and eye $(\mathrm{H}) . \mathrm{HuC}$ is expressed in neurons throughout the brain and eye in wild-type (I). HuC is expressed in the brain in mutant; however it is not as organized as wild-type and only remnants of $\mathrm{HuC}$ are detected in mutant eye. (J) S100 $\beta$ labels astrocytes in the brain and eyes in wild-type $(\mathrm{K})$, but is greatly reduced and sporadic in the brain and eyes of mutant (L). Scale bars are $50 \mu \mathrm{m}$. 


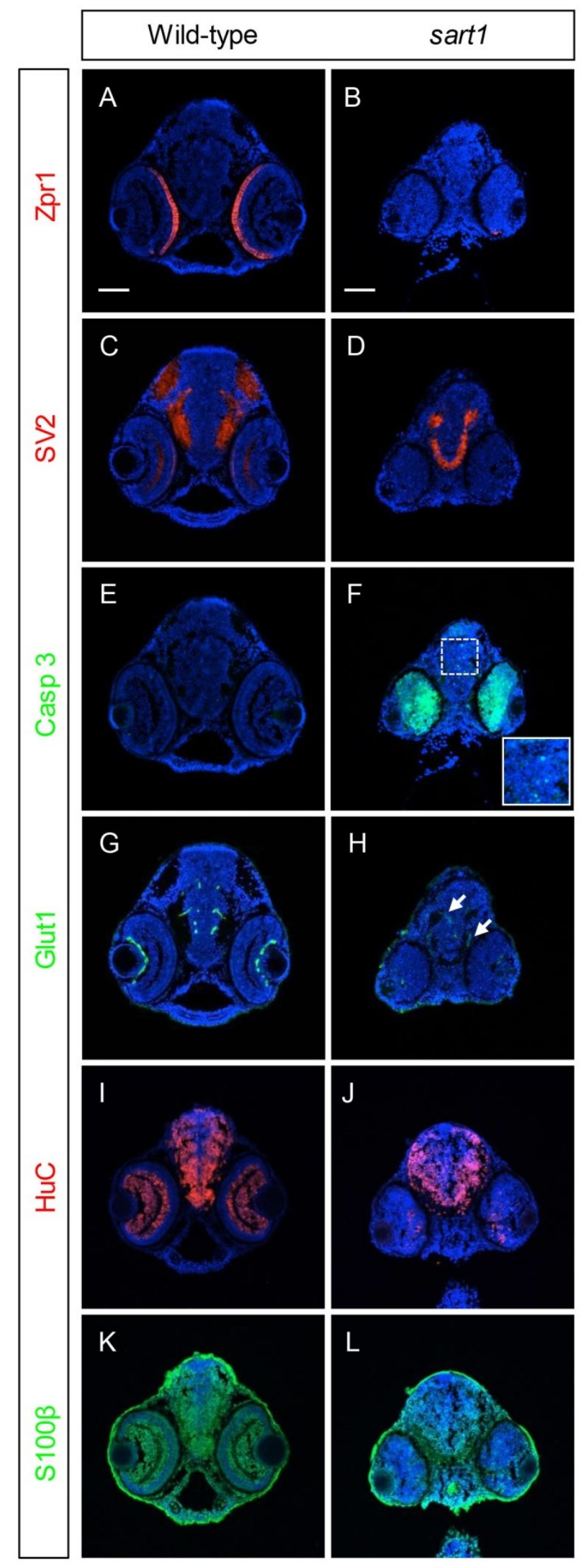




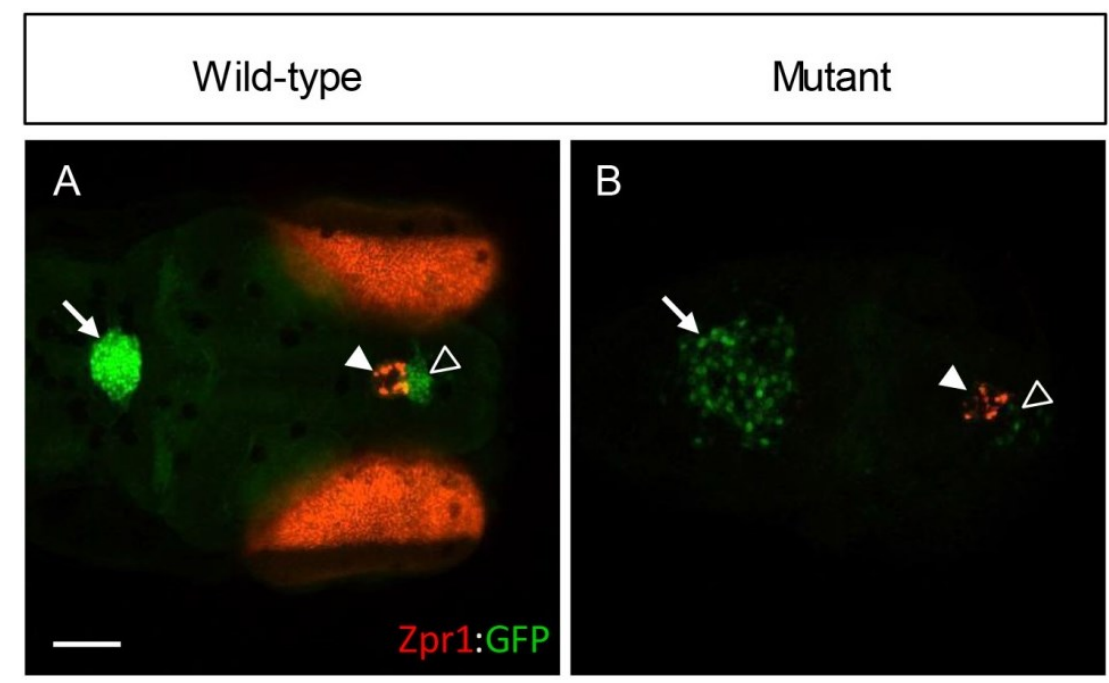

Figure 5-4. Zpr1 Is Expressed in the Pineal Gland in cp27.5 Mutants.

(A) Wild-type cp27.5 larvae at 4 dpf with GFP (green) in the dCP (open arrowhead) and $\mathrm{mCP}$ (arrow) and Zpr1 expression (red) in the pineal gland (filled arrowhead) and eyes. (B) Mutant $c p 27.5$ larvae at $4 \mathrm{dpf}$ with GFP (green) in the dCP (open arrowhead) and $\mathrm{mCP}$ (arrow) and Zpr1 expression (red) in the pineal gland (filled arrowhead). Mutants have no Zpr1 expression in the eye. dCP, diencephalic choroid plexus; $\mathrm{mCP}$, myelencephalic choroid plexus. Scale bar is $50 \mu \mathrm{m}$. 


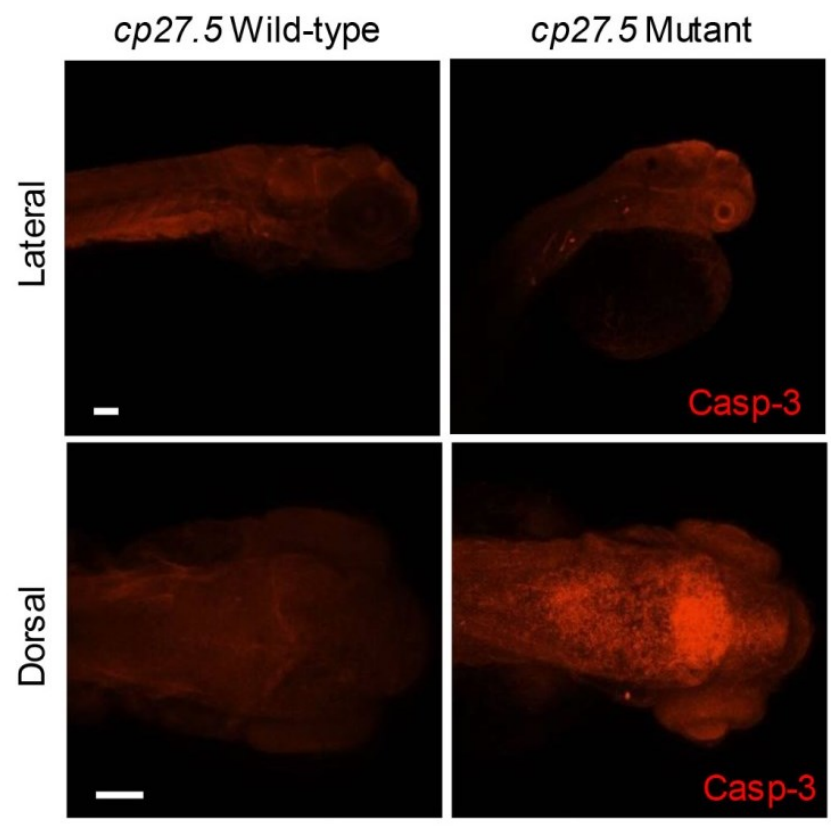

Figure 5-5. Activated Caspase-3 Is Expressed in cp27.5 Mutant Brain.

Whole mount IHC with activated Caspase-3 antibody reveals expression in the dorsal midline and eye in $c p 27.5$ mutants (right column). Activated Caspase-3 was not detected in cp27.5 wild-type (left column). Scale bars are $50 \mu \mathrm{m}$. 


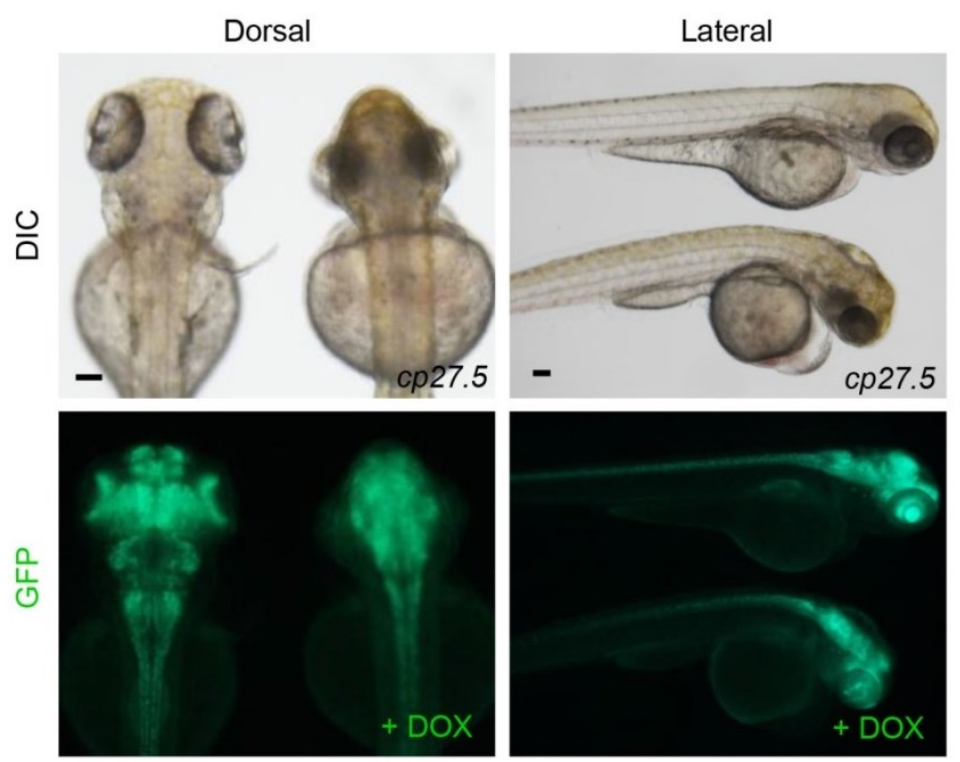

Figure 5-6. Doxycycline Induces GFP in the CNS of $c p 27.5$ Larvae.

cp27.5 larvae treated with doxycycline induces the TetON system using the $\mathrm{HuC}$ promoter resulting in GFP expression throughout the CNS. Wild-type larvae (shown on left and upper sides) express GFP in the brain, spinal cord, and eyes. Mutant larvae (shown right and bottom sides) express GFP in the brain and spinal cord but little to no expression in the eyes. Scale bars are $50 \mu \mathrm{m}$. 
rhodamine, and a 40-kDa fluorescein dextran. While the 3-kDa and 10-kDa tracers leaked into the brain ventricle in wild-type, it did not penetrate into the brain itself or surrounding areas. In mutants, these tracers entered the brain ventricle, but they were not confined to the ventricle and leaked into nearby tissues (Figure 5-7A-D). In wild-type, the 40-kDa tracer was retained within the vasculature (Figure 5-7E-F). Because this tracer remained within the blood vessels, we were able to observe reduced vasculature throughout the brain and abnormal vascular patterning around the eye in $c p 27.5$ mutants. However, this phenotype was restricted only to these regions. Vascular patterning and permeability along the tail was normal (Figure 5-7G-H).

\section{RNA-Seq Analysis Identifies Up-Regulated Apoptotic Genes}

To determine what genes are affected by this specific mutation in sart1, we performed an RNA-Seq analysis and looked for up or down-regulation of genes throughout the zebrafish genome by comparing cp27.5 wild-type and mutant RNA expression levels. The complete analysis is found in Appendix B. SupplementalFile1. Because Sart1 is involved in spliceosome assembly, a process that occurs ubiquitously in all cell types, we expected to identify dysregulation of genes involved in a number of processes. Because the entire RNA-Seq data is too extensive to be described here, we have reported in the following paragraphs only the genes we confirmed to be up or down-regulated by qRT-PCR (Table 5-2). The fold change normalized to $\beta$-actin for each gene is found in Figure 5-8. For a more widespread look at the genes analyzed by RNA-Seq and their relative expression, please refer to Table B-2.

From the RNA-Seq analysis, we identified up-regulation of sart1 in mutants, thus confirming the whole mount in situ hybridization data showing elevated sartl expression in the brain compared to wild-type. As mentioned previously, sart1 has been demonstrated to be involved in apoptosis where it appears to play a duel role by inducing cell cycle arrest(Hosokawa et al., 2005), but also aiding in drug resistance in cancer cells (Laetsch et al., 2014). One of the more highly up-regulated genes was tp53. While the tp53 transcript was up-regulated, we were also interested to see whether p53 protein was increased. By performing Western blot analysis, we observed that both wild-type and mutant had a faint band at the predicted protein size of $42 \mathrm{kDa}$. However in cp27.5 mutants, a larger and highly expressed band was detected closer to $50 \mathrm{kDa}$, and another band, while not as prominent, was detected to be greater than $50 \mathrm{kDa}$. These bands are faintly present in wild-type samples perhaps due to heterozygous siblings in the wild-type pool (Figure 5-9). We also observed an increase in $m d m 2$ expression, which acts as an inhibitor of tp53 by ubiquitinating p53 and targeting it for degradation. Alternatively, a feedback loop can occur where p53 can in turn activate Mdm2 (Zhao et al., 2014). During cellular stress, the cell death pathway is initiated causing p53 levels to increase and Mdm2 levels to decrease. As a result of decreased Mdm2, p53 initiates $m d m 2$ transcription. As Mdm2 levels then increase, Mdm2 in turn acts to inhibit p53 (Zhao et al., 2014). In addition, we detected increased expression of another interesting gene clusterin (clu). Studies in zebrafish have observed clusterin expression specifically 


\section{Figure 5-7. Fluorescent Tracer Injections Reveal Increased Permeability in Mutants.}

cp27.5 wild-type and mutant larvae were intravenously injected with a mixture of a 3-kDa Cascade blue dextran, 10-kDa Rhodamine dextran, and 40-kDa Fluorescein dextran at 2 dpf. (A) Wild-type injected with 3-kDa Cascade blue dextran has tracer permeable to the brain ventricle (arrow), but is restricted from the brain and eye (asterisks). (B) Mutant injected with 3-kDa Cascade blue dextran has tracer retained in the brain ventricle (arrow), but also leaks into the surrounding tissue (asterisks). (C) Wild-type injected with 10-kDa Rhodamine dextran has tracer permeable to the brain ventricle (arrow), but is also restricted from the brain and eye (asterisks). However, the $10-\mathrm{kDa}$ Rhodamine dextran is more restricted to the brain vasculature compared to the 3-kDa Cascade blue (arrowhead). (D) Mutant injected with 10-kDa Rhodamine dextran has tracer permeable to the brain ventricle (arrow), but also leaks into the surrounding tissue (asterisks) and is not restricted to the brain vasculature as seen in wild-type (arrowhead). (E) Wild-type injected with 40-kDa Fluorescein dextran has little to no tracer leakage into the brain ventricle (arrow) and is restricted from entering the brain and eye (asterisk). The tracer is also retained within the brain vasculature (arrowhead). (F) Mutant injected with 40-kDa Fluorescein dextran has tracer slightly permeable to the brain ventricle (arrow), but not as severe compared to the 3-kDa Cascade blue and $10-\mathrm{kDa}$ Rhodamine dextrans. The tracer does not appear to leak into the brain, eye, or surrounding tissue as severely as the smaller tracers; however, there is still absence of the tracer retained within the brain vasculature (arrowhead). (G) Merged image of wild-type larvae co-injected with all three tracers. $(\mathrm{H})$ Merged image of mutant larvae co-injected with all three tracers. Tracers are retained within the peripheral tail vasculature in mutants comparable to wild-type (arrowheads). Scale bars are $50 \mu \mathrm{m}$. 


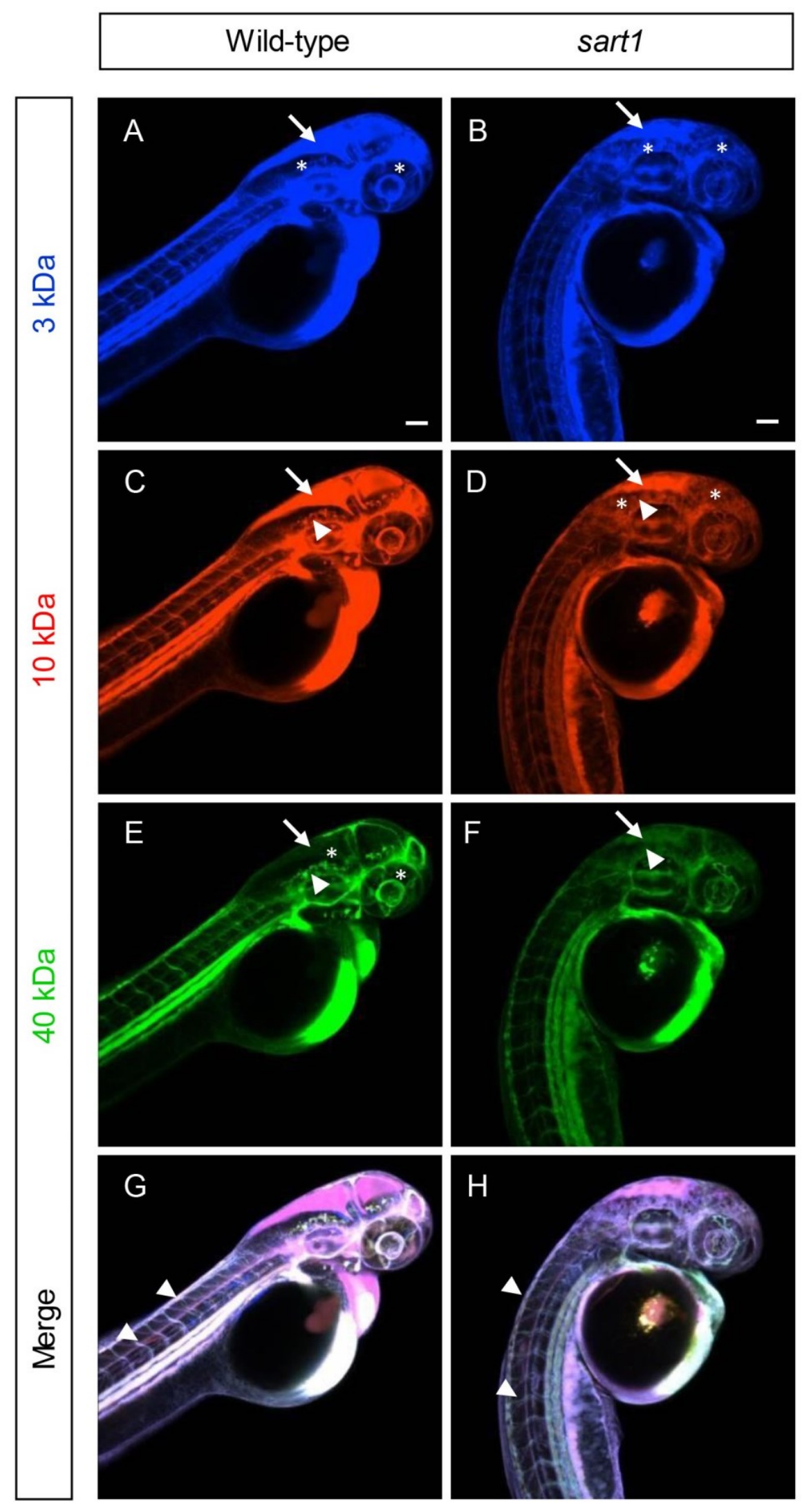


Table 5-2. Up-Regulated and Down-Regulated Genes Confirmed by qRT-PCR.

\begin{tabular}{lcccc}
\hline Gene & Accession Number & $\begin{array}{c}\mathbf{l}_{2} \text { (MUT-WT) } \\
\text { from RNA-Seq }\end{array}$ & $\begin{array}{c}\text { Fold Increase } \\
\text { from } \\
\text { qRT-PCR }\end{array}$ & $\begin{array}{c}\text { Normalized } \\
\text { for } \\
\text { qRT-PCR }\end{array}$ \\
\hline sart1 & NM_001002673 & 3.35 & 1.64 & 0.64 \\
tp53 & NM_131327 & 4.22 & 3.14 & 2.14 \\
mdm2 & NM_131364 & 4.07 & 2.99 & 1.99 \\
clu & NM_200802 & 3.76 & 3.61 & 2.62 \\
cldn5a & NM_213274 & 3.42 & 1.20 & 0.20 \\
mmp9 & NM_213123 & 8.38 & 61.99 & 61.01 \\
prpf31 & NM_200504 & 3.71 & 2.75 & 1.76 \\
lsm7 & NM_001048006 & 3.71 & 3.22 & 2.23 \\
pde6h & NM_200785 & -16.92 & 0.00 & -1.00 \\
opn1sw1 & NM_131319 & -7.29 & 0.00 & -1.00 \\
crx & NM_152940 & -1.71 & 0.05 & -0.95 \\
actb1 & NM131031 & 0.71 & 1.00 & 0.00 \\
\hline
\end{tabular}




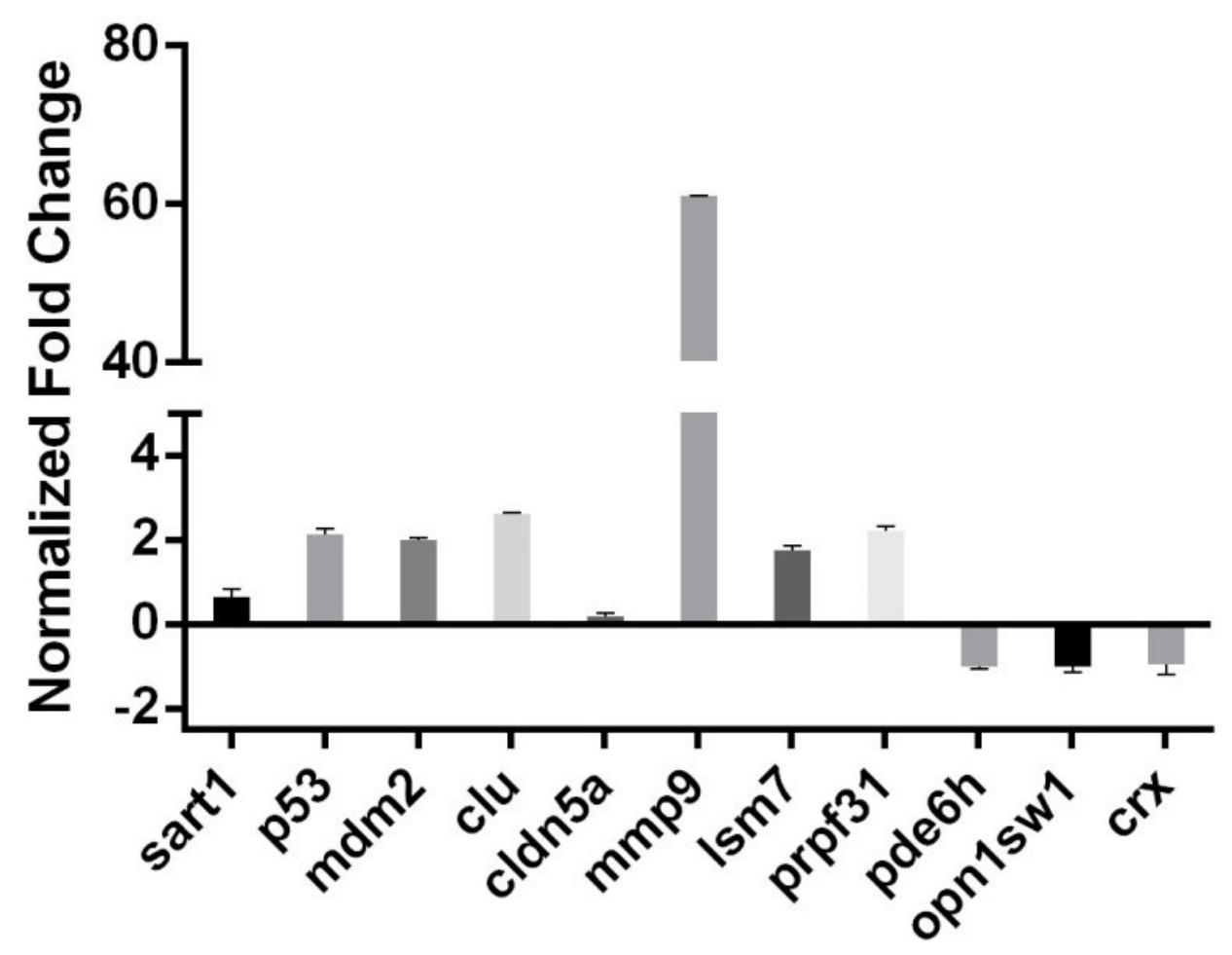

Figure 5-8. qRT-PCR Confirms Up-Regulated or Down-Regulated Genes from RNA-Seq Analysis.

RNA-Seq analysis was confirmed using qRT-PCR. Fold changes were normalized to $\beta$-actin which was set to zero. The majority of genes are up-regulated including sart1, apoptotic genes (tp53, $m d m 2$, clu), tight junctions ( $c l d n 5 a)$, extracellular matrix enzymes $($ mmp9), and spliceosome factors ( $l s m 7, \operatorname{prpf} 31)$, while vision-related genes are down-regulated (pde6h, opn 1sw1, crx). Measurements are expressed as mean $\pm \mathrm{SE}$ (standard error) for $n=3$ from mutant samples. 


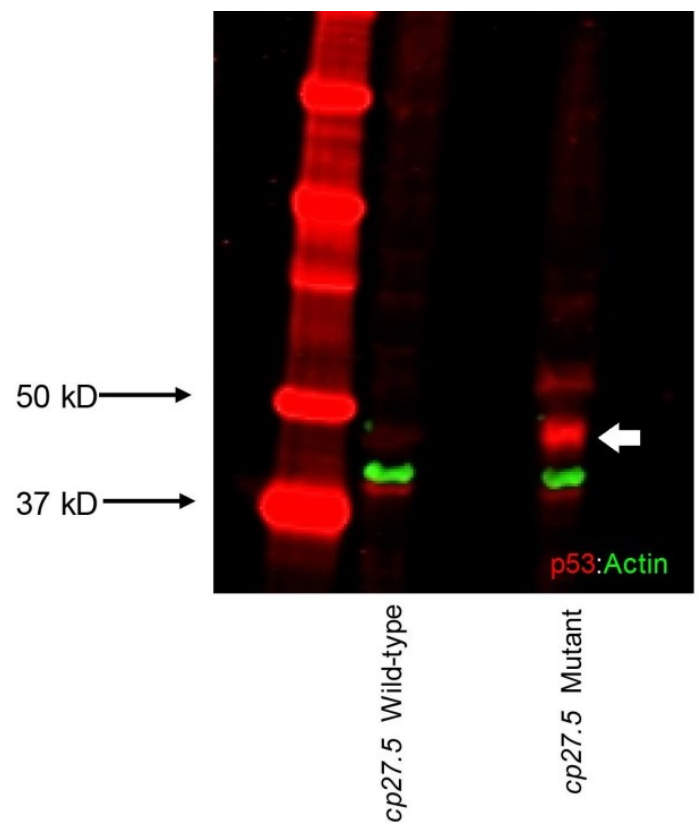

Figure 5-9. Western Blot Analysis Reveals a Larger, More Highly Expressed p53 in cp27.5 Mutant.

Both $c p 27.5$ wild-type and mutant express p53 with a protein size of $42 \mathrm{kDa}$ (red). However, $c p 27.5$ mutants also highly express a p53 protein that is absent in wild-type (arrow). Protein levels were normalized to $\beta$-actin (green). 
within the choroid plexus (Jiao et al., 2011). It acts as a chaperone and is a pro-survival gene in its secreted form by binding to Bax and preventing Bax from traveling to the mitochondria (Trougakos et al., 2009). In its nuclear form, which originates from alternative splicing, it acts as a pro-death gene by promoting cell death in a Caspase-3 independent manner (Leskov et al., 2003; Trougakos et al., 2009).

\section{RNA-Seq Analysis Identifies Up-Regulated Spliceosome Components}

Previous studies have identified increased expression of spliceosome transcripts when another component of the spliceosome itself, or a protein involved in spliceosome assembly is defective (Chen et al., 2014; Jia et al., 2012). Because Sart1 is involved in the recruitment of U4/U6.U5 tri-snRNP to the spliceosome, we wanted to observe whether additional factors involved in spliceosome assembly were also defective or up-regulated in order to compensate for possible defects in Sart1 function. The first gene we confirmed by qRT-PCR was prpf31. This gene is a member of the precursor $R N A$ processing $(P R P)$ genes and similar to sart1, it also plays a role in recruiting U4/U6.U5 tri-snRNP to the spliceosome (Vithana et al., 2001). Interestingly, mutations of prpf31 are found in patients with RP, a degenerative disease of photoreceptors in the eye (Vithana et al., 2001). We determined that prpf31 was up-regulated in cp27.5 mutants. We also observed up-regulation of a gene from another class of spliceosome components, lsm7. Lsm7, which binds to U6 in the spliceosome is a member of a family of proteins called Sm proteins that are also involved in assembling major spliceosome factors (He and Parker, 2000).

\section{RNA-Seq Analysis Identifies Up-Regulated Claudin 5a and Matrix Metalloproteinase Protein (MMP9)}

In characterizing cp 27.5 mutants, we also observed defects in Cldn5 protein expression. Whole mount IHC showed Cldn5 expression on the surface of the brain ventricle in mutants, however, we did not observe co-localization with $\mathrm{CPe}$ as seen in wild-type larvae (Figure 5-10). The results also suggested increased Cldn5 protein in mutants. Based on RNA-Seq and qRT-PCR data, we observed increased expression of cldn5a transcript in mutant, indicating that protein expression in the brain ventricle may also be up-regulated. In a recent study, $m m p 9$ was suggested to lead to Cldn5 degradation at the BCSFB allowing for leukocytes entry into the CSF (Chiu and Lai, 2013). mmp9 is also said to be up-regulated in the $\mathrm{CP}$ in the presence of inflammation (Kratzer et al., 2013). We observed a significant increase in mmp9 expression in cp27.5 mutants; however, whether up-regulation is specific to the BCSFB requires further investigation. 


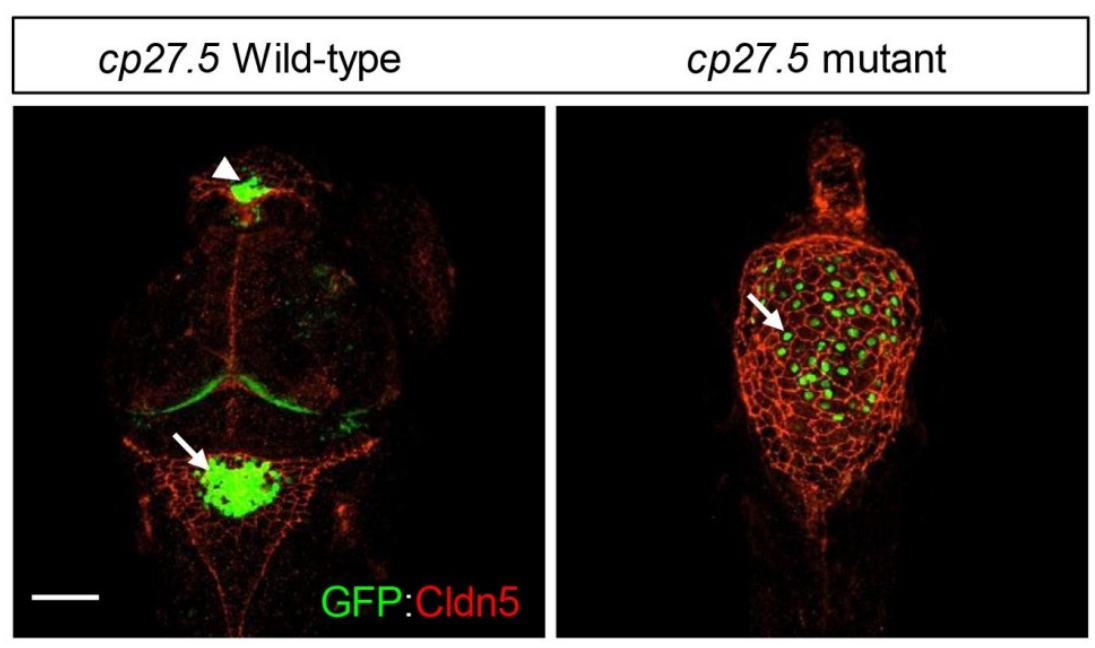

Figure 5-10. Claudin 5 Is Expressed in cp27.5 Mutants, but Does Not Co-Localize with Choroid Plexus Epithelia.

Whole mount IHC was performed on cp27.5 wild-type and mutant larvae at $3 \mathrm{dpf}$ In wild-type, the dCP (arrowhead) and mCP (arrow) express GFP (green). Claudin 5 (red) is localized to the surface vasculature and co-localizes with the CP epithelia (yellow). In mutant, the $\mathrm{dCP}$ is absent and the mCP consists of individual sporadic GFP positive cells (arrow). Claudin 5 is expressed throughout the brain ventricle and surrounds $\mathrm{CP}$ epithelia, but does not co-localize with the cells as seen in wild-type. Scale bar is $50 \mu \mathrm{m}$. 


\section{RNA-Seq Analysis Identifies Down-Regulation of Vision-Related Genes}

The final class of genes we confirmed by qRT-PCR were those specific to the eye. Because retinal degeneration has been shown as a result of splicing defects or mutations in spliceosome components (Chen et al., 2014; Tanackovic et al., 2011), we were interested to see if mutations in sartl led to decreased expression of vision-related genes. Cone-rod homeobox (crx) is a transcription factor found in photoreceptor cells and mutations in this gene have been associated with cone-rod dystrophy (Freund et al., 1997). We observed a dramatic decrease in crx expression, which is not surprising due to our IHC data showing a loss of photoreceptors in $c p 27.5$ mutants. We also looked at phosphodiesterase $6 H$ (pde6h), a gene that encodes for the $\gamma$ (gamma) subunit of a cyclic guanosine monophosphate phosphodiesterase specific to cone photoreceptors. Mutations in this gene have been associated with achromatopsia, which is an inherited retinal dystrophy (Kohl et al., 2012). pde6h was also down-regulated in cp27.5 mutants. The final gene we analyzed was opsin-1, short-wave-sensitive 1 (opn 1sw1), a gene specific to zebrafish expressed in ultraviolet cone photoreceptors (Aluru et al., 2014). Opsins found in cone photoreceptors belong to G-protein-coupled receptors (GPCRs). These receptors are important in light absorption so that the signal can be relayed to activate downstream components such as G-proteins, transducin, and phosphodiesterases (Aluru et al., 2014). Again, not surprisingly, due to its specific expression in the retina, opn 1swl was down-regulated in $c p 27.5$ mutants. While here we provide a brief overview of specific genes identified from the RNA-Seq analysis and their response to the sart1 mutation, future studies will examine in more depth the role of sartl in regulating the expression levels and patterning of these genes. Suggested roles for sart 1 are described in the Discussion below.

\section{Discussion}

The current study describes a zebrafish mutant from cp27.5 with a point mutation in the splicing component sart1. In addition to splicing, sart1 has been implicated in a number of different processes such as cell cycle arrest and apoptosis and has been suggested as a target for gene therapy due to its activation of apoptosis and its role as an antigen recognized by CTLs in certain types of cancer (Hosokawa et al., 2005). More recently, sart1 has been shown to contribute to drug resistance in cancer cells (Allen et al., 2012; Laetsch et al., 2014). The goal of this study was to characterize mutant sart1 and determine what other genes are affected due to this mutation. In doing so, we plan to discover the role of sart 1 in overall cellular processes such as cell death; in addition to better understanding its traditional role at the transcriptional level in splicing.

One of the earliest phenotypes detected in these mutants was forebrain necrosis at approximately late $2 \mathrm{dpf}$. Prior to this time point, mutants were undistinguishable from wild-type siblings. The maternally derived transcript identified by RT-PCR was present on day zero and may explain why we were unable to isolate mutant embryos prior to 2 dpf due to the contribution of wild-type sartl from the mother. Interestingly, by whole mount in situ hybridization and RNA-Seq analysis we determined that the point mutation 
in sartl results in an obvious up-regulation of the transcript. Up-regulation of mutated splicing genes have been demonstrated in other studies such as prpf4, a gene encoding a protein that interacts with the U4/U6 di-snRNP and stabilizes the complex (Chen et al., 2014). A mutation from a proline to leucine in prpf4 led to its increased expression and also up-regulation of other splicing factors including sart1. Similar to our study, they also detected a loss of photoreceptors in the zebrafish eye and a down-regulation of vision related genes such as opnllwl, another opsin gene (Chen et al., 2014).

The loss of protein expression in genes found in the eye, such as Zpr1, SV2, HuC, and $\mathrm{S} 100 \beta$, is not surprising due to previous studies showing that mutations in splicing factors can result in photoreceptor degeneration in diseases such as RP (Chen et al., 2014) where the most noticeable phenotype is a loss of photoreceptor cells (Mordes et al., 2006). A recent study cited that six genes related to splicing have been found to have mutations in patients with RP including PRPF31, PRPF8, PRPF3, PAP-1, SNRNP200, and $P R P F 6$ and that these mutations are responsible for autosomal dominant RP in more than $12 \%$ of cases (Benaglio et al., 2014). Of these genes, PRPF8, PRPF31, and PRPF3 are involved in forming and recruiting the U4/U6.U5 tri-snRNP to the spliceosome (Mordes et al., 2006). The exact cause of how mutations in splicing genes result in RP is still unknown; however, several models or hypotheses have been suggested. Because photoreceptor cells require a high level of mRNA and protein synthesis due to a high turnover rate in cell types such as the rod outer segment, a haploinsufficiency model explains how losing a functional copy of the gene results in decreased mRNA processing and therefore decreased protein resulting in degeneration (Mordes et al., 2006). Another hypothesis is that these mutations are specific only to photoreceptor cells and that the spliceosome is never activated in these cells due to mutations in genes essential for forming and recruiting the complex to pre-mRNA (Mordes et al., 2006). How this splicing defect is specific to photoreceptors is proposed in a third model where it is believed that cofactors specific to photoreceptors act alongside the spliceosome to regulate splicing; however specific factors found only in photoreceptors have yet to be identified (Mordes et al., 2006). A final hypothesis is that these mutations have a toxic effect on the cells due to a gain-of-function where the mutated splicing genes interfere with normal splicing in wild-type copies resulting in increased defective splicing and cell death even if the wild-type gene is present (Mordes et al., 2006).

A similar hypothesis was suggested by Jia et al. where mis-splicing due to mutations in U2, a major spliceosome component, results in a buildup of mis-spliced mRNA that translates into dysfunctional proteins. Another hypothesis by Jia et al. proposes that introns retained within the mRNA sequence cause the spliceosome to remain at the intron rather than be released, resulting in alternative splicing and a toxic feedback loop where mRNA continues to be mis-spliced and cell death occurs (Jia et al., 2012). In addition to splicing factors, mutations in other genes such as $c r x$ encoding a transcription factor, and $p d e$ genes involved in phototransduction, both of which we identified as down-regulated in $c p 27.5$, also contribute to RP (Mordes et al., 2006).

In cp27.5 mutants, we detected increased cell death within the eye as observed by activated Caspase 3 antibody staining. However, from the RNA-Seq data, we also 
detected up-regulation of $t p 53$ and $m d m 2$. While up-regulation of tp53 explains the increased cell death found in the eye and brain, increased expression of $m d m 2$ may indicate that it is trying to compensate for increased tp53 expression. Alternatively, because of p53 up-regulation, Mdm2 may also be overexpressed due to the ability of p53 to activate its expression. Future studies will investigate whether Mdm2 is functional in inhibiting p53 protein and whether p53 is functional and activating Mdm2 and forming a negative feedback loop. By Western blot, we were able to detect an alternative p53 protein synthesized in $c p 27.5$ mutants. Based on these findings, we suggest that alternative splicing due to mutated sart1, causes additional p53 proteins to be synthesized in cp 27.5 mutants that cannot be inhibited by Mdm2 thereby resulting in increased cell death (Figure 5-11). As to why cell death is localized to the eye and brain has yet to be determined.

While we observed defects primarily related to the eye, sart 1 was majorly expressed in the brain as demonstrated by whole mount in situ hybridization. Because mRNA splicing occurs in every cell type, spliceosome genes are presumed to be ubiquitously expressed. However, recent studies have suggested that these genes may be temporally or spatially regulated. A previous study in mice with a U2 mutation resulted in the retention of introns in genes such as the cell adhesion molecule L1CAM, and caused specific phenotypes, such as neurodegeneration within the cerebellum (Jia et al., 2012). Similar to our findings in the zebrafish eye, mice also had activated Caspase- 3 protein expression in granule cells of the cerebellum. This study identified that the mutant gene was most highly expressed in the cerebellum compared to other tissues (Jia et al., 2012). This phenomenon may also occur in cp27.5 mutants as we detected a dramatic increase in sart 1 mRNA within the mutant brain compared to wild-type. We have supported this claim by demonstrating that this increase in expression is due to up-regulation of the transcript rather than increased permeability of the probe since deltaC expression was decreased in mutant.

cp27.5 was originally identified from a genetic screen isolating mutants with CP developmental defects. Interestingly, one of the genes we identified from the RNA-Seq data was clusterin. This gene has been demonstrated in recent studies to be expressed specifically within the zebrafish CP in early development (Jiao et al., 2011). A more detailed description of this study was presented on pg. 16. This finding is relevant to our analysis in that the nuclear form of clusterin has also been described to play a role in cell death (Leskov et al., 2003; Trougakos et al., 2009). Future studies will investigate whether the nuclear form of clusterin is localized to the CP and contributes to cell death in cp27.5 mutants since the CP has dispersed GFP positive cells and never fully develops. Up-regulation of clusterin from the RNA-Seq analysis may prove this hypothesis to be true since alternative splicing of clusterin may cause inhibitors to no longer prevent its expression. A lack of clusterin inhibition would then result in its overexpression and increased cell death. Alternatively, as mentioned before, the secreted form of Clusterin can act as a pro-survival protein (Trougakos et al., 2009). If the secreted protein is up-regulated in mutants, Clusterin may be acting in competition with pro-apoptotic genes such as p53 and compensating for cell death in the CP. However, even though clusterin 


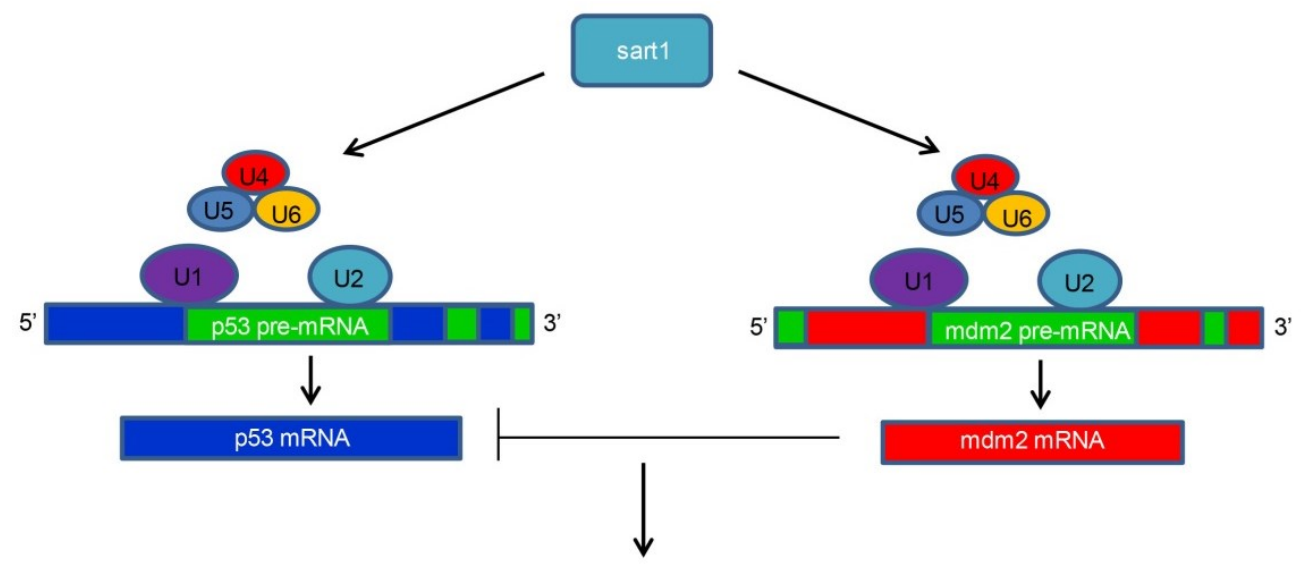

Cell survival $\rightarrow$ photoreceptors present

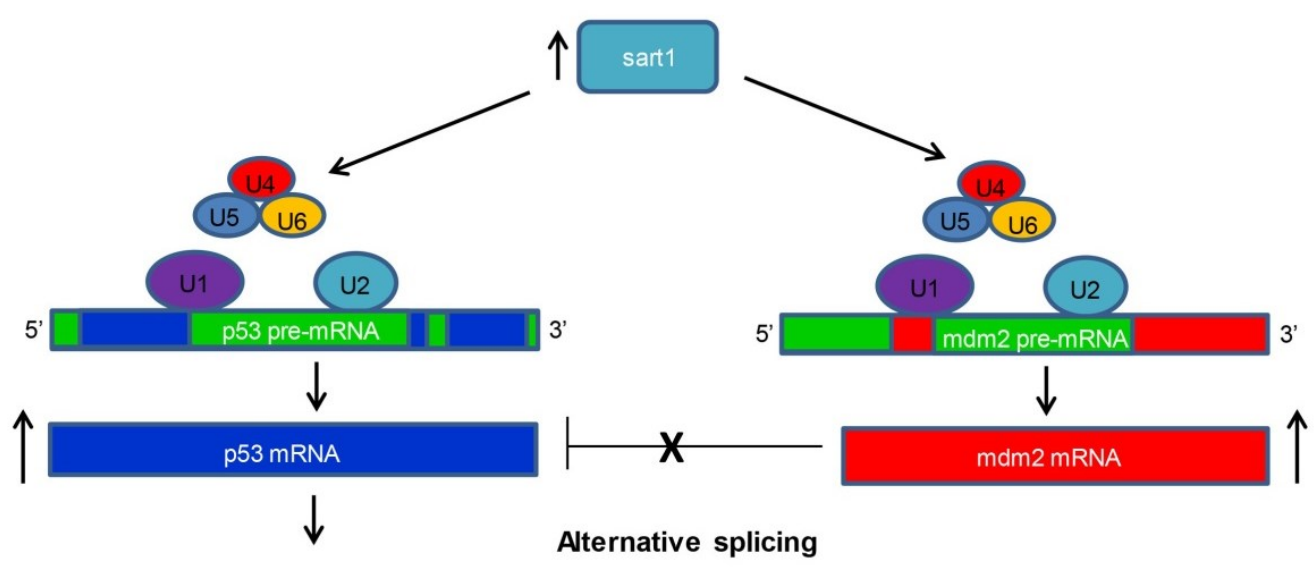

Cell death $\rightarrow$ photoreceptors absent

Figure 5-11. Schematic Diagram for the Hypothetical Role of sart1 in Apoptosis.

In wild-type, Sart1 acts to recruit the U4/U6.U5 tri-snRNP to the spliceosome complex where U1 and U2 are already bound to pre-mRNA at the 5' splice site and branch point site, respectively. Once the spliceosome is assembled, it processes pre mRNA by removing introns and producing mature mRNA transcripts for genes such as tp53 and $m d m 2$. Mdm2 inhibits p53 allowing for cell survival of photoreceptors. In mutant, while the sart 1 transcript is up-regulated, altered Sart1 protein may result in defective recruitment of U4/U6.U5 to the spliceosome thereby causing alternative splicing of $p 53$ and $m d m 2$. Alternative splicing may result in altered proteins such as Mdm2 which can no longer function to inhibit p53 activity. In addition, alternatively spliced p53 transcripts may result in proteins that can no longer be inhibited by Mdm2. This phenomenon may lead to up-regulation of p53 and increased cell death in photoreceptors. 
was found to be up-regulated at the transcript level, future studies will need to determine whether the functional protein is also increased.

Another protein we demonstrated to be expressed in the CP was Cldn5. From the RNA-Seq data, we determined that $c l d n 5 a$ is also slightly up-regulated in $c p 27.5$ mutants. While we were able to detect Cldn5 expression in mutants by whole mount IHC, future experiments will need to determine if this protein is actually functional. Cldn5 appeared to be mis-localized in mutants due to an absence of its expression overlapping with GFP in $\mathrm{CPe}$. Another hypothesis is that a disruption of anchoring proteins that tether tight junctions to the plasma membrane, such as ZO-1, may also be disrupted resulting is mislocalization of Cldn5 to the cell membrane. Additional evidence for defects in Cldn5 was demonstrated by injecting fluorescent dextrans intravenously into cp27.5 larvae.

Due to the developmental time point for analysis at $2 \mathrm{dpf}$, both wild-type and mutants had leakage into the brain ventricle since the CP had not yet developed. However, mutants demonstrated increased permeability outside the ventricle where smaller tracers leaked into surrounding tissues suggesting defects in tight junctions. Tracer studies also revealed abnormal vasculature in the CNS indicating that the sart1 mutation may strictly be CNS specific and not affecting tight junctions or vasculature of the periphery. $m m p 9$ was also very highly up-regulated as confirmed by qRT-PCR. As mentioned previously, mmp9 can result in the degradation of Claudin 5 at the BCSFB resulting in increased barrier permeability (Chiu and Lai, 2013). The same phenomena may result as a downstream effect of mutated sart 1 where overexpression of $m m p 9$ causes mis-localization of Claudin 5 and increased barrier permeability and leakiness.

Interestingly the previous study mentions that up-regulation of $m m p 9$ was dependent on the NF- $\kappa$ B pathway (Chiu and Lai, 2013). We identified from the RNA-Seq data that nfkbiaa (nuclear factor of kappa light polypeptide gene enhancer in $B$-cells inhibitor, alpha $a$ ), a component of the NF- $\kappa$ B pathway, is one of the top up-regulated genes in mutant (see Table B-2). This gene is also known as IkappaBalpha and acts as an inhibitor of the NF- $\kappa \mathrm{B}$ signaling pathway (Correa et al., 2004). Again, this may indicate compensatory mechanisms as a result of the sart1 mutation where inhibitors of the NF- $\kappa \mathrm{B}$ pathway attempt to reduce MMP9 activity and restore Cldn5 to its normal expression.

While the genetic pathways downstream of sart1 in cp27.5 mutants have yet to be determined, the current study has revealed dysregulation of genes involved in apoptosis, splicing, vision, tight junctions, and matrix metalloproteinases. RNA-Seq analysis has provided substantial insights into genes and potential pathways regulated by sart1. This study and others have demonstrated that mutations in splicing machinery or proteins associated with the spliceosome have widespread affects in a number of signaling processes in addition to splicing defects. Also, differences between phenotypes observed in the CNS versus periphery may suggest that sart1 regulates the expression of certain genes in a tissue-specific manner. 


\section{Conclusion}

In conclusion, sart1 is a fascinating gene whose complex expression and function will provide interesting insights into cellular pathways for years to come. We have discovered a zebrafish model with a mutation in sart1 that will aid in these future investigations. By performing RNA-Seq, we have already identified numerous genes affected by this mutation. Using IHC and Western blot, we have also identified defects in protein expression in a number of genes including those specific to brain and eye. Future studies will aim to answer the outstanding question of how genes expressed in multiple cell types, such as splicing-related genes, result in defects or abnormalities in a spatially or temporally tissue or cell-specific manner. 


\section{CHAPTER 6. SUMMARY AND CONCLUSIONS}

The current study had three primary goals: 1) to establish zebrafish as functional model to study CP development, 2) to perform a forward genetic screen identifying CP mutants, and 3) to discover the gene responsible for the phenotypes identified in cp27.5.

For the first aim, we have supplemented previous studies describing $\mathrm{CP}$ development in zebrafish by generating an enhancer trap line expressing EGFP within $\mathrm{CPe}$. This line we termed Et(cp:EGFP) ${ }^{s j 2}$ allowed us to monitor CP formation over time and investigate its functional properties. While we have demonstrated that zebrafish provide a suitable model for observing this process in a living system due to its rapid development and transparent nature in early stages, we have also shown that the zebrafish $\mathrm{CP}$ possess barrier properties and functions comparable to other vertebrates. The CNS has long been known for its barrier components including tight junctions and transporters localized to the cell membrane. These proteins create both a physical and chemical barrier, respectively, allowing for CNS homeostasis and protection from insult. Here, we have shown that the zebrafish $\mathrm{CP}$ expresses the tight junction protein, Claudin 5 and its anchoring protein ZO-1. While additional tight junctions have been shown to be highly expressed in the $\mathrm{CP}$, antibodies against these proteins in zebrafish are difficult to acquire or do not exist. With gaining popularity of the zebrafish model in recent years, access to commercially available antibodies for zebrafish may continue to increase allowing for additional tight junction proteins in the $\mathrm{CP}$ to be identified.

In addition to tight junctions, we provided evidence for transporter activity at the BCSFB. While we were not able to identify antibodies that labelled transporter proteins in the $\mathrm{CP}$, we did observe accumulation of fluorescent dyes including Rhodamine 123 and Fluorescein. These dyes are known substrates of transporters expressed in the CP such as Mrp1 and Oat3. By injecting these tracers intravenously, we observed that the dyes can be transported from the fenestrated blood vasculature into the CPe signifying transporter localization at the basolateral surface of the epithelia. Alternatively, by injecting tracers directly into the CSF in the brain ventricle, we observed accumulation in the $\mathrm{CP}$ suggesting that transporters are also localized to the apical membrane of $\mathrm{CPe}$. Future studies will identify specific transporter expression at the $\mathrm{CP}$ and determine their localization within the cell membrane.

We also determined when the BCSFB forms in zebrafish. By injecting fluorescent tracers conjugated to different molecular weight dextrans, we observed that the BCSFB acquires size-selective properties as it develops and forms by 4 dpf. Using fluorescent tracers attached to various sized dextrans allowed for physical barrier properties to be determined since these molecules are transported between cells, not through them. This characterization study using the Et(cp:EGFP $)^{s j 2}$ line has paved the way for future endeavors investigating the genes and signaling pathways essential for $\mathrm{CP}$ development, function, and maintenance by providing an in vivo model system with a BCSFB similar to other vertebrates and the ability to monitor barrier development over time. 
Zebrafish are also exceptional models for forward genetic screening. After characterizing the $\mathrm{CP}$ in zebrafish, we performed a genetic analysis by mutagenizing the $E t(c p: E G F P)^{s j 2}$ line and screened for mutants based on abnormalities in EGFP expression or patterning in the $\mathrm{mCP}$. We identified a number of spontaneous mutants with abnormalities outside of the $\mathrm{CP}$ such as heart and eye edema or smaller head size. However, for some of these mutants the CP developed normally. Alternatively, we identified spontaneous mutants having normal overall development, but no CP formation. Isolating these classes of spontaneous mutants suggests that specific genetic pathways are governing $\mathrm{CP}$ development, function, and maintenance. We also identified five classes of recessive mutants following Mendelian genetics with approximately $25 \%$ of the offspring containing $\mathrm{CP}$ abnormalities. While these mutants were screened initially for defects in CP development, we observed additional phenotypes such as small head and eyes, heart and eye edema, or no swimbladder.

We were interested to determine whether the mutated genes from these lines were specific to the $\mathrm{CP}$ or whether they were ubiquitously expressed genes promoting global defects. We performed genetic mapping experiments for the line $c p 27.5$ due to its severe brain ventricle expansion in addition to $\mathrm{CP}$ defects. We hypothesized that the gene may be specific to CSF production or regulation due to the expanded ventricle phenotype. Using traditional genetic mapping strategies such as positional cloning, we identified the mutation on Chromosome 21. Since the sequencing of the zebrafish genome, positional cloning has become easier to define a critical interval due to polymorphic markers designed throughout the genome. However, this strategy is often time consuming by sequencing multiple candidate genes and can be difficult to identify a map position based on recombination frequency. For this study, we also employed an innovative approach using WES that identified the gene mutation based on isolating homozygous regions in the mutant genome. Using this strategy, we identified a point mutation in sart1, a gene involved in spliceosome assembly. We confirmed that sartl was the gene responsible for the abnormalities observed in sartl mutants by performing rescue experiments that restored the most of the mutants to normal or near normal phenotypes.

In characterizing cp27.5 mutants by in situ hybridization and RNA-Seq analysis, we identified that a $\mathrm{G}$ to A point mutation at the donor site of exon 12 results in up-regulation of sart1 specifically in the brain. Increased expression of sart 1 resulted in dysregulation of genes involved in a number of pathways and processes such as apoptotic and vision-related genes, and genes important for cell-cell interactions and splicing. Future studies will investigate the mechanisms of sartl in regulating the expression levels and patterning of these genes and determine whether it plays specific role in $\mathrm{CP}$ development. 


\section{LIST OF REFERENCES}

Abbott, N.J., Khan, E.U., Rollinson, C.M., Reichel, A., Janigro, D., Dombrowski, S.M., Dobbie, M.S., and Begley, D.J. (2002). Drug resistance in epilepsy: the role of the blood-brain barrier. Novartis Foundation symposium 243, 38-47; discussion 47-53, 180-185.

Abbott, N.J., Patabendige, A.A., Dolman, D.E., Yusof, S.R., and Begley, D.J. (2010). Structure and function of the blood-brain barrier. Neurobiology of disease 37, 13-25.

Abdelilah-Seyfried, S. (2010). Claudin-5a in developing zebrafish brain barriers: another brick in the wall. BioEssays : news and reviews in molecular, cellular and developmental biology $32,768-776$.

Allen, W.L., Stevenson, L., Coyle, V.M., Jithesh, P.V., Proutski, I., Carson, G., Gordon, M.A., Lenz, H.J., Van Schaeybroeck, S., Longley, D.B., et al. (2012). A systems biology approach identifies SART1 as a novel determinant of both 5-fluorouracil and SN38 drug resistance in colorectal cancer. Molecular cancer therapeutics 11, 119-131.

Allende, M.L., Amsterdam, A., Becker, T., Kawakami, K., Gaiano, N., and Hopkins, N. (1996). Insertional mutagenesis in zebrafish identifies two novel genes, pescadillo and dead eye, essential for embryonic development. Genes \& development 10, 3141-3155.

Aluru, N., Jenny, M.J., and Hahn, M.E. (2014). Knockdown of a zebrafish aryl hydrocarbon receptor repressor (AHRRa) affects expression of genes related to photoreceptor development and hematopoiesis. Toxicological sciences : an official journal of the Society of Toxicology 139, 381-395.

Alvira-Botero, X., and Carro, E.M. (2010). Clearance of amyloid-beta peptide across the choroid plexus in Alzheimer's disease. Current aging science 3, 219-229.

Amsterdam, A., Yoon, C., Allende, M., Becker, T., Kawakami, K., Burgess, S., Gaiano, N., and Hopkins, N. (1997). Retrovirus-mediated insertional mutagenesis in zebrafish and identification of a molecular marker for embryonic germ cells. Cold Spring Harbor symposia on quantitative biology $62,437-450$.

Anderson, J.M., and Van Itallie, C.M. (2009). Physiology and function of the tight junction. Cold Spring Harbor perspectives in biology 1 , a002584.

Angelow, S., Zeni, P., and Galla, H.J. (2004). Usefulness and limitation of primary cultured porcine choroid plexus epithelial cells as an in vitro model to study drug transport at the blood-CSF barrier. Advanced drug delivery reviews 56, 1859-1873.

Arunachalam, M., Raja, M., Vijayakumar, C., Malaiammal, P., and Mayden, R.L. (2013). Natural history of zebrafish (Danio rerio) in India. Zebrafish 10, 1-14. 
Aslamkhan, A.G., Thompson, D.M., Perry, J.L., Bleasby, K., Wolff, N.A., Barros, S., Miller, D.S., and Pritchard, J.B. (2006). The flounder organic anion transporter fOat has sequence, function, and substrate specificity similarity to both mammalian Oat1 and Oat3. American journal of physiology Regulatory, integrative and comparative physiology 291, R1773-1780.

Baehr, C.H., Fricker, G., and Miller, D.S. (2006). Fluorescein-methotrexate transport in dogfish shark (Squalus acanthias) choroid plexus. American journal of physiology Regulatory, integrative and comparative physiology 291, R464-472.

Bally-Cuif, L., Cholley, B., and Wassef, M. (1995). Involvement of Wnt-1 in the formation of the mes/metencephalic boundary. Mechanisms of development 53, 23-34.

Bamshad, M.J., Ng, S.B., Bigham, A.W., Tabor, H.K., Emond, M.J., Nickerson, D.A., and Shendure, J. (2011). Exome sequencing as a tool for Mendelian disease gene discovery. Nature reviews Genetics 12, 745-755.

Barichello, T., Generoso, J.S., Simoes, L.R., Elias, S.G., and Quevedo, J. (2013). Role of oxidative stress in the pathophysiology of pneumococcal meningitis. Oxidative medicine and cellular longevity 2013,371465.

Bell, R.D., and Zlokovic, B.V. (2009). Neurovascular mechanisms and blood-brain barrier disorder in Alzheimer's disease. Acta neuropathologica 118, 103-113.

Benaglio, P., San Jose, P.F., Avila-Fernandez, A., Ascari, G., Harper, S., Manes, G., Ayuso, C., Hamel, C., Berson, E.L., and Rivolta, C. (2014). Mutational screening of splicing factor genes in cases with autosomal dominant retinitis pigmentosa. Molecular vision 20,843-851.

Bill, B.R., Balciunas, D., McCarra, J.A., Young, E.D., Xiong, T., Spahn, A.M., Garcia-Lecea, M., Korzh, V., Ekker, S.C., and Schimmenti, L.A. (2008). Development and Notch signaling requirements of the zebrafish choroid plexus. PloS one 3, e3114.

Blader, P., and Strahle, U. (2000). Zebrafish developmental genetics and central nervous system development. Human molecular genetics 9, 945-951.

Bowen, M.E., Henke, K., Siegfried, K.R., Warman, M.L., and Harris, M.P. (2012). Efficient mapping and cloning of mutations in zebrafish by low-coverage whole-genome sequencing. Genetics 190, 1017-1024.

Bronger, H., Konig, J., Kopplow, K., Steiner, H.H., Ahmadi, R., Herold-Mende, C., Keppler, D., and Nies, A.T. (2005). ABCC drug efflux pumps and organic anion uptake transporters in human gliomas and the blood-tumor barrier. Cancer research 65 , $11419-11428$.

Broom, E.R., Gilthorpe, J.D., Butts, T., Campo-Paysaa, F., and Wingate, R.J. (2012). The roof plate boundary is a bi-directional organiser of dorsal neural tube and choroid plexus development. Development 139, 4261-4270. 
Brown, P.D., Davies, S.L., Speake, T., and Millar, I.D. (2004). Molecular mechanisms of cerebrospinal fluid production. Neuroscience 129, 957-970.

Brown, R.C., and Davis, T.P. (2002). Calcium modulation of adherens and tight junction function: a potential mechanism for blood-brain barrier disruption after stroke. Stroke; a journal of cerebral circulation 33, 1706-1711.

Burckhardt, G., and Wolff, N.A. (2000). Structure of renal organic anion and cation transporters. American journal of physiology Renal physiology 278, F853-866.

Chang, G. (2003). Multidrug resistance ABC transporters. FEBS letters 555, 102-105.

Chen, C.P., Chen, R.L., and Preston, J.E. (2012). The influence of ageing in the cerebrospinal fluid concentrations of proteins that are derived from the choroid plexus, brain, and plasma. Experimental gerontology 47, 323-328.

Chen, R.L., Chen, C.P., and Preston, J.E. (2010). Elevation of CSF albumin in old sheep: relations to CSF turnover and albumin extraction at blood-CSF barrier. Journal of neurochemistry 113, 1230-1239.

Chen, X., Liu, Y., Sheng, X., Tam, P.O., Zhao, K., Chen, X., Rong, W., Liu, Y., Liu, X., Pan, X., et al. (2014). PRPF4 mutations cause autosomal dominant retinitis pigmentosa. Human molecular genetics 23, 2926-2939.

Chiu, P.S., and Lai, S.C. (2013). Matrix metalloproteinase-9 leads to claudin-5 degradation via the NF-kappaB pathway in BALB/c mice with eosinophilic meningoencephalitis caused by Angiostrongylus cantonensis. PloS one 8, e53370.

Chodobski, A., and Szmydynger-Chodobska, J. (2001). Choroid plexus: target for polypeptides and site of their synthesis. Microscopy research and technique 52, 65-82.

Choe, Y., Siegenthaler, J.A., and Pleasure, S.J. (2012). A cascade of morphogenic signaling initiated by the meninges controls corpus callosum formation. Neuron 73 , 698-712.

Correa, R.G., Tergaonkar, V., Ng, J.K., Dubova, I., Izpisua-Belmonte, J.C., and Verma, I.M. (2004). Characterization of NF-kappa B/I kappa B proteins in zebra fish and their involvement in notochord development. Molecular and cellular biology 24, 5257-5268.

Cullen, M., Elzarrad, M.K., Seaman, S., Zudaire, E., Stevens, J., Yang, M.Y., Li, X., Chaudhary, A., Xu, L., Hilton, M.B., et al. (2011). GPR124, an orphan G protein-coupled receptor, is required for CNS-specific vascularization and establishment of the blood-brain barrier. Proceedings of the National Academy of Sciences of the United States of America 108, 5759-5764.

Currle, D.S., Cheng, X., Hsu, C.M., and Monuki, E.S. (2005). Direct and indirect roles of CNS dorsal midline cells in choroid plexus epithelia formation. Development 132, 3549-3559. 
Daneman, R., and Rescigno, M. (2009). The gut immune barrier and the blood-brain barrier: are they so different? Immunity $31,722-735$.

Daoud, R., Kast, C., Gros, P., and Georges, E. (2000). Rhodamine 123 binds to multiple sites in the multidrug resistance protein (MRP1). Biochemistry 39, 15344-15352.

de Bruijn, E., Cuppen, E., and Feitsma, H. (2009). Highly Efficient ENU Mutagenesis in Zebrafish. Methods in molecular biology 546, 3-12.

Decimo, I., Fumagalli, G., Berton, V., Krampera, M., and Bifari, F. (2012). Meninges: from protective membrane to stem cell niche. American journal of stem cells 1, 92-105.

Deeley, R.G., Westlake, C., and Cole, S.P. (2006). Transmembrane transport of endo- and xenobiotics by mammalian ATP-binding cassette multidrug resistance proteins. Physiological reviews 86, 849-899.

Del Bigio, M.R. (1995). The ependyma: a protective barrier between brain and cerebrospinal fluid. Glia 14, 1-13.

Dermietzel, R., Krause, D., Kremer, M., Wang, C., and Stevenson, B. (1992). Pattern of glucose transporter (Glut 1) expression in embryonic brains is related to maturation of blood-brain barrier tightness. Developmental dynamics : an official publication of the American Association of Anatomists 193, 152-163.

Dobin, A., Davis, C.A., Schlesinger, F., Drenkow, J., Zaleski, C., Jha, S., Batut, P., Chaisson, M., and Gingeras, T.R. (2013). STAR: ultrafast universal RNA-seq aligner. Bioinformatics 29, 15-21.

Dohrmann, G.J. (1970). The choroid plexus: a historical review. Brain research 18, 197-218.

Dragunow, M. (2013). Meningeal and choroid plexus cells--novel drug targets for CNS disorders. Brain research 1501, 32-55.

Driever, W., Solnica-Krezel, L., Schier, A.F., Neuhauss, S.C., Malicki, J., Stemple, D.L., Stainier, D.Y., Zwartkruis, F., Abdelilah, S., Rangini, Z., et al. (1996). A genetic screen for mutations affecting embryogenesis in zebrafish. Development 123, 37-46.

Dziegielewska, K.M., Ek, J., Habgood, M.D., and Saunders, N.R. (2001). Development of the choroid plexus. Microscopy research and technique 52, 5-20.

Ek, C.J., Habgood, M.D., Dziegielewska, K.M., and Saunders, N.R. (2003). Structural characteristics and barrier properties of the choroid plexuses in developing brain of the opossum (Monodelphis Domestica). The Journal of comparative neurology 460, 451-464.

Ellis, H.M., and Horvitz, H.R. (1986). Genetic control of programmed cell death in the nematode C. elegans. Cell 44, 817-829. 
Emerich, D.F., Skinner, S.J., Borlongan, C.V., Vasconcellos, A.V., and Thanos, C.G. (2005). The choroid plexus in the rise, fall and repair of the brain. BioEssays : news and reviews in molecular, cellular and developmental biology 27, 262-274.

English, D.P., and Santin, A.D. (2013). Claudins Overexpression in Ovarian Cancer: Potential Targets for Clostridium Perfringens Enterotoxin (CPE) Based Diagnosis and Therapy. International journal of molecular sciences 14, 10412-10437.

Fairfield, H., Gilbert, G.J., Barter, M., Corrigan, R.R., Curtain, M., Ding, Y., D'Ascenzo, M., Gerhardt, D.J., He, C., Huang, W., et al. (2011). Mutation discovery in mice by whole exome sequencing. Genome biology 12, R86.

Flicek, P., Amode, M.R., Barrell, D., Beal, K., Billis, K., Brent, S., Carvalho-Silva, D., Clapham, P., Coates, G., Fitzgerald, S., et al. (2014). Ensembl 2014. Nucleic acids research 42, D749-755.

Freund, C.L., Gregory-Evans, C.Y., Furukawa, T., Papaioannou, M., Looser, J., Ploder, L., Bellingham, J., Ng, D., Herbrick, J.A., Duncan, A., et al. (1997). Cone-rod dystrophy due to mutations in a novel photoreceptor-specific homeobox gene (CRX) essential for maintenance of the photoreceptor. Cell 91, 543-553.

Furuse, M., Sasaki, H., and Tsukita, S. (1999). Manner of interaction of heterogeneous claudin species within and between tight junction strands. The Journal of cell biology 147, 891-903.

Gaiano, N., Amsterdam, A., Kawakami, K., Allende, M., Becker, T., and Hopkins, N. (1996). Insertional mutagenesis and rapid cloning of essential genes in zebrafish. Nature $383,829-832$.

Garcia-Garcia, M.J., Eggenschwiler, J.T., Caspary, T., Alcorn, H.L., Wyler, M.R., Huangfu, D., Rakeman, A.S., Lee, J.D., Feinberg, E.H., Timmer, J.R., et al. (2005). Analysis of mouse embryonic patterning and morphogenesis by forward genetics. Proceedings of the National Academy of Sciences of the United States of America 102, 5913-5919.

Garcia-Lecea, M., Kondrychyn, I., Fong, S.H., Ye, Z.R., and Korzh, V. (2008). In vivo analysis of choroid plexus morphogenesis in zebrafish. PloS one 3, e3090.

Gato, A., Martin, P., Alonso, M.I., Martin, C., Pulgar, M.A., and Moro, J.A. (2004). Analysis of cerebro-spinal fluid protein composition in early developmental stages in chick embryos. Journal of experimental zoology Part A, Comparative experimental biology 301, 280-289.

Goldsmith, J.R., and Jobin, C. (2012). Think small: zebrafish as a model system of human pathology. Journal of biomedicine \& biotechnology 2012, 817341 . 
Gonzalez, A.M., Podvin, S., Lin, S.Y., Miller, M.C., Botfield, H., Leadbeater, W.E., Roberton, A., Dang, X., Knowling, S.E., Cardenas-Galindo, E., et al. (2011). Ecrg4 expression and its product augurin in the choroid plexus: impact on fetal brain development, cerebrospinal fluid homeostasis and neuroprogenitor cell response to CNS injury. Fluids and barriers of the CNS 8,6 .

Gonzalez, A.M., Taylor, W.M., Johanson, C.E., King, J.C., Leadbeater, W.E., Stopa, E.G., and Baird, A. (2010). Co-localization and regulation of basic fibroblast growth factor and arginine vasopressin in neuroendocrine cells of the rat and human brain. Cerebrospinal fluid research 7, 13.

Greenwood, S., Swetloff, A., Wade, A.M., Terasaki, T., and Ferretti, P. (2008). Fgf2 is expressed in human and murine embryonic choroid plexus and affects choroid plexus epithelial cell behaviour. Cerebrospinal fluid research 5, 20.

Grove, E.A., Tole, S., Limon, J., Yip, L., and Ragsdale, C.W. (1998). The hem of the embryonic cerebral cortex is defined by the expression of multiple Wnt genes and is compromised in Gli3-deficient mice. Development 125, 2315-2325.

Grunwald, D.J., and Eisen, J.S. (2002). Headwaters of the zebrafish -- emergence of a new model vertebrate. Nature reviews Genetics 3, 717-724.

Gu, H., Zhong, Z., Jiang, W., Du, E., Dodel, R., Liu, J., Farlow, M.R., Zheng, W., and $\mathrm{Du}, \mathrm{Y}$. (2014). The role of choroid plexus in IVIG-induced beta-amyloid clearance. Neuroscience 270, 168-176.

Gunzel, D., and Yu, A.S. (2013). Claudins and the modulation of tight junction permeability. Physiological reviews 93, 525-569.

Hasselblatt, M. (2009). Ependymal tumors. Recent results in cancer research Fortschritte der Krebsforschung Progres dans les recherches sur le cancer 171, 51-66.

He, W., and Parker, R. (2000). Functions of Lsm proteins in mRNA degradation and splicing. Current opinion in cell biology 12, 346-350.

Henke, K., Bowen, M.E., and Harris, M.P. (2013). Perspectives for identification of mutations in the zebrafish: making use of next-generation sequencing technologies for forward genetic approaches. Methods 62, 185-196.

Horvitz, H.R. (1999). Genetic control of programmed cell death in the nematode Caenorhabditis elegans. Cancer research 59, 1701s-1706s.

Hosokawa, M., Kadota, R., Shichijo, S., Itoh, K., Dmitriev, I., Krasnykh, V., Curiel, D.T., Takue, Y., Wakasugi, H., Takashima, S., et al. (2005). Cell cycle arrest and apoptosis induced by SART-1 gene transduction. Anticancer research 25, 1983-1990. 
Hrabe de Angelis, M.H., Flaswinkel, H., Fuchs, H., Rathkolb, B., Soewarto, D., Marschall, S., Heffner, S., Pargent, W., Wuensch, K., Jung, M., et al. (2000).

Genome-wide, large-scale production of mutant mice by ENU mutagenesis. Nature genetics 25, 444-447.

Huang, D.W., Sherman, B.T., Lempicki, R.A. (2009a). Bioinformatics enrichment tools: paths toward the comprehensive functional analysis of large gene lists. Nucleic acids research $37,1-13$.

Huang, D.W., Sherman, B.T., Lempicki, R.A. (2009b). Systematic and integrative analysis of large gene lists using DAVID Bioinformatics Resources. Nature Protoc 4, 44-57.

Huang, X., Ketova, T., Fleming, J.T., Wang, H., Dey, S.K., Litingtung, Y., and Chiang, C. (2009). Sonic hedgehog signaling regulates a novel epithelial progenitor domain of the hindbrain choroid plexus. Development 136, 2535-2543.

Ide, C., Kitada, M., Chakrabortty, S., Taketomi, M., Matsumoto, N., Kikukawa, S., Mizoguchi, A., Kawaguchi, S., Endoh, K., and Suzuki, Y. (2001). Grafting of choroid plexus ependymal cells promotes the growth of regenerating axons in the dorsal funiculus of rat spinal cord: a preliminary report. Experimental neurology 167, 242-251.

Imayoshi, I., Shimogori, T., Ohtsuka, T., and Kageyama, R. (2008). Hes genes and neurogenin regulate non-neural versus neural fate specification in the dorsal telencephalic midline. Development 135, 2531-2541.

Irvin, D.K., Nakano, I., Paucar, A., and Kornblum, H.I. (2004). Patterns of Jagged1, Jagged2, Delta-like 1 and Delta-like 3 expression during late embryonic and postnatal brain development suggest multiple functional roles in progenitors and differentiated cells. Journal of neuroscience research 75, 330-343.

Jansen, W.F., de Weger, R.A., Woutersen, R.A., van Loveren, H., and van de Kamer, J.C. (1976). The saccus dorsalis of the rainbow trout, Salmo gairdneri Richardson:

histological, cytochemical, electron microscopical and autoradiographical observations. Cell and tissue research 167, 467-491.

Janssen, S.F., van der Spek, S.J., Ten Brink, J.B., Essing, A.H., Gorgels, T.G., van der Spek, P.J., Jansonius, N.M., and Bergen, A.A. (2013). Gene expression and functional annotation of the human and mouse choroid plexus epithelium. PloS one 8 , e83345.

Jeong, J.Y., Kwon, H.B., Ahn, J.C., Kang, D., Kwon, S.H., Park, J.A., and Kim, K.W. (2008). Functional and developmental analysis of the blood-brain barrier in zebrafish. Brain research bulletin 75, 619-628.

Jia, Y., Mu, J.C., and Ackerman, S.L. (2012). Mutation of a U2 snRNA gene causes global disruption of alternative splicing and neurodegeneration. Cell 148, 296-308. 
Jiao, S., Dai, W., Lu, L., Liu, Y., Zhou, J., Li, Y., Korzh, V., and Duan, C. (2011). The conserved clusterin gene is expressed in the developing choroid plexus under the regulation of notch but not IGF signaling in zebrafish. Endocrinology 152, 1860-1871.

Johanson, C.E., Stopa, E.G., and McMillan, P.N. (2011). The blood-cerebrospinal fluid barrier: structure and functional significance. Methods in molecular biology 686, 101-131.

Johansson, P.A., Irmler, M., Acampora, D., Beckers, J., Simeone, A., and Gotz, M. (2013). The transcription factor Otx2 regulates choroid plexus development and function. Development 140, 1055-1066.

Jorgensen, E.M., and Mango, S.E. (2002). The art and design of genetic screens: caenorhabditis elegans. Nature reviews Genetics 3, 356-369.

Jurica, M.S., and Moore, M.J. (2003). Pre-mRNA splicing: awash in a sea of proteins. Molecular cell 12, 5-14.

Keep, R.F., and Smith, D.E. (2011). Choroid plexus transport: gene deletion studies. Fluids and barriers of the CNS $8,26$.

Kettleborough, R.N., Busch-Nentwich, E.M., Harvey, S.A., Dooley, C.M., de Bruijn, E., van Eeden, F., Sealy, I., White, R.J., Herd, C., Nijman, I.J., et al. (2013). A systematic genome-wide analysis of zebrafish protein-coding gene function. Nature 496, 494-497.

Kile, B.T., and Hilton, D.J. (2005). The art and design of genetic screens: mouse. Nature reviews Genetics 6, 557-567.

Kimmel, C.B., Hatta, K., and Eisen, J.S. (1991). Genetic control of primary neuronal development in zebrafish. Development Suppl 2, 47-57.

Klepper, J., and Voit, T. (2002). Facilitated glucose transporter protein type 1 (GLUT1) deficiency syndrome: impaired glucose transport into brain-- a review. European journal of pediatrics $161,295-304$.

Knott, G.W., Dziegielewska, K.M., Habgood, M.D., Li, Z.S., and Saunders, N.R. (1997). Albumin transfer across the choroid plexus of South American opossum (Monodelphis domestica). The Journal of physiology 499 ( Pt 1), 179-194.

Kohl, S., Coppieters, F., Meire, F., Schaich, S., Roosing, S., Brennenstuhl, C., Bolz, S., van Genderen, M.M., Riemslag, F.C., European Retinal Disease, C., et al. (2012). A nonsense mutation in PDE6H causes autosomal-recessive incomplete achromatopsia. American journal of human genetics 91, 527-532.

Korzh, V. (2007). Transposons as tools for enhancer trap screens in vertebrates. Genome biology 8 Suppl 1, S8. 
Kratzer, I., Liddelow, S.A., Saunders, N.R., Dziegielewska, K.M., Strazielle, N., and Ghersi-Egea, J.F. (2013). Developmental changes in the transcriptome of the rat choroid plexus in relation to neuroprotection. Fluids and barriers of the CNS 10, 25.

Kratzer, I., Vasiljevic, A., Rey, C., Fevre-Montange, M., Saunders, N., Strazielle, N., and Ghersi-Egea, J.F. (2012). Complexity and developmental changes in the expression pattern of claudins at the blood-CSF barrier. Histochemistry and cell biology 138, 861-879.

Krause, G., Winkler, L., Piehl, C., Blasig, I., Piontek, J., and Muller, S.L. (2009). Structure and function of extracellular claudin domains. Annals of the New York Academy of Sciences 1165, 34-43.

Laetsch, T.W., Liu, X., Vu, A., Sliozberg, M., Vido, M., Elci, O.U., Goldsmith, K.C., and Hogarty, M.D. (2014). Multiple components of the spliceosome regulate Mcl1 activity in neuroblastoma. Cell death \& disease 5, e1072.

Lagaraine, C., Skipor, J., Szczepkowska, A., Dufourny, L., and Thiery, J.C. (2011). Tight junction proteins vary in the choroid plexus of ewes according to photoperiod. Brain research $1393,44-51$.

Lambert, J.C., Heath, S., Even, G., Campion, D., Sleegers, K., Hiltunen, M., Combarros, O., Zelenika, D., Bullido, M.J., Tavernier, B., et al. (2009). Genome-wide association study identifies variants at CLU and CR1 associated with Alzheimer's disease. Nature genetics 41, 1094-1099.

Lander, E.S., and Waterman, M.S. (1988). Genomic mapping by fingerprinting random clones: a mathematical analysis. Genomics 2, 231-239.

Leskov, K.S., Klokov, D.Y., Li, J., Kinsella, T.J., and Boothman, D.A. (2003). Synthesis and functional analyses of nuclear clusterin, a cell death protein. The Journal of biological chemistry 278, 11590-11600.

Li, Z.S., Dziegielewska, K.M., and Saunders, N.R. (1997). Transthyretin distribution in the developing choroid plexus of the South American opossum (Monodelphis domestica). Cell and tissue research 287, 621-624.

Liddelow, S.A. (2011). Fluids and barriers of the CNS: a historical viewpoint. Fluids and barriers of the CNS 8,2 .

Liddelow, S.A., Dziegielewska, K.M., Ek, C.J., Johansson, P.A., Potter, A.M., and Saunders, N.R. (2009). Cellular transfer of macromolecules across the developing choroid plexus of Monodelphis domestica. The European journal of neuroscience 29, 253-266. 
Liddelow, S.A., Dziegielewska, K.M., VandeBerg, J.L., Noor, N.M., Potter, A.M., and Saunders, N.R. (2011). Modification of protein transfer across blood/cerebrospinal fluid barrier in response to altered plasma protein composition during development. The European journal of neuroscience 33, 391-400.

Liddelow, S.A., Dziegielewska, K.M., Vandeberg, J.L., and Saunders, N.R. (2010). Development of the lateral ventricular choroid plexus in a marsupial, Monodelphis domestica. Cerebrospinal fluid research 7, 16.

Liddelow, S.A., Temple, S., Mollgard, K., Gehwolf, R., Wagner, A., Bauer, H., Bauer, H.C., Phoenix, T.N., Dziegielewska, K.M., and Saunders, N.R. (2012). Molecular characterisation of transport mechanisms at the developing mouse blood-CSF interface: a transcriptome approach. PloS one 7, e33554.

Liebner, S., Corada, M., Bangsow, T., Babbage, J., Taddei, A., Czupalla, C.J., Reis, M., Felici, A., Wolburg, H., Fruttiger, M., et al. (2008). Wnt/beta-catenin signaling controls development of the blood-brain barrier. The Journal of cell biology 183, 409-417.

Lim, D.A., Tramontin, A.D., Trevejo, J.M., Herrera, D.G., Garcia-Verdugo, J.M., and Alvarez-Buylla, A. (2000). Noggin antagonizes BMP signaling to create a niche for adult neurogenesis. Neuron 28, 713-726.

Lippoldt, A., Liebner, S., Andbjer, B., Kalbacher, H., Wolburg, H., Haller, H., and Fuxe, K. (2000). Organization of choroid plexus epithelial and endothelial cell tight junctions and regulation of claudin- $1,-2$ and -5 expression by protein kinase C. Neuroreport 11 , 1427-1431.

Lobo, I. (2008). Pleiotropy: One gene can affect multiple traits. Nature Education 1.

Louvi, A., Yoshida, M., and Grove, E.A. (2007). The derivatives of the Wnt3a lineage in the central nervous system. The Journal of comparative neurology 504, 550-569.

Lowery, L.A., De Rienzo, G., Gutzman, J.H., and Sive, H. (2009). Characterization and classification of zebrafish brain morphology mutants. Anatomical record 292, 94-106.

Lowery, L.A., Rubin, J., and Sive, H. (2007). Whitesnake/sfpq is required for cell survival and neuronal development in the zebrafish. Developmental dynamics : an official publication of the American Association of Anatomists 236, 1347-1357.

Lowery, L.A., and Sive, H. (2005). Initial formation of zebrafish brain ventricles occurs independently of circulation and requires the nagie oko and snakehead/atplala.1 gene products. Development 132, 2057-2067.

Mack, J., Squier, W., and Eastman, J.T. (2009). Anatomy and development of the meninges: implications for subdural collections and CSF circulation. Pediatric radiology $39,200-210$. 
Makarova, O.V., Makarov, E.M., and Luhrmann, R. (2001). The 65 and $110 \mathrm{kDa}$ SR-related proteins of the U4/U6.U5 tri-snRNP are essential for the assembly of mature spliceosomes. The EMBO journal 20, 2553-2563.

Marques, F., Sousa, J.C., Coppola, G., Gao, F., Puga, R., Brentani, H., Geschwind, D.H., Sousa, N., Correia-Neves, M., and Palha, J.A. (2011). Transcriptome signature of the adult mouse choroid plexus. Fluids and barriers of the CNS $8,10$.

Matera, A.G., and Wang, Z. (2014). A day in the life of the spliceosome. Nature reviews Molecular cell biology 15, 108-121.

Meinke, D.W., and Sussex, I.M. (1979). Embryo-lethal mutants of Arabidopsis thaliana. A model system for genetic analysis of plant embryo development. Developmental biology 72, 50-61.

Miller, A.C., Obholzer, N.D., Shah, A.N., Megason, S.G., and Moens, C.B. (2013). RNA-seq-based mapping and candidate identification of mutations from forward genetic screens. Genome research 23, 679-686.

Moller, G. (2002). The Nobel Prize in Physiology or Medicine for 2002. Scandinavian journal of immunology 56,435 .

Monnot, A.D., and Zheng, W. (2013). Culture of choroid plexus epithelial cells and in vitro model of blood-CSF barrier. Methods in molecular biology 945, 13-29.

Moore, J.L., Rush, L.M., Breneman, C., Mohideen, M.A., and Cheng, K.C. (2006). Zebrafish genomic instability mutants and cancer susceptibility. Genetics 174, 585-600.

Mordes, D., Luo, X., Kar, A., Kuo, D., Xu, L., Fushimi, K., Yu, G., Sternberg, P., Jr., and Wu, J.Y. (2006). Pre-mRNA splicing and retinitis pigmentosa. Molecular vision 12, $1259-1271$.

Mortazavi, M.M., Griessenauer, C.J., Adeeb, N., Deep, A., Bavarsad Shahripour, R., Loukas, M., Tubbs, R.I., and Tubbs, R.S. (2014). The choroid plexus: a comprehensive review of its history, anatomy, function, histology, embryology, and surgical considerations. Child's nervous system : ChNS : official journal of the International Society for Pediatric Neurosurgery 30, 205-214.

Moser, A.R., Pitot, H.C., and Dove, W.F. (1990). A dominant mutation that predisposes to multiple intestinal neoplasia in the mouse. Science 247, 322-324.

Muto, A., Orger, M.B., Wehman, A.M., Smear, M.C., Kay, J.N., Page-McCaw, P.S., Gahtan, E., Xiao, T., Nevin, L.M., Gosse, N.J., et al. (2005). Forward genetic analysis of visual behavior in zebrafish. PLoS genetics 1 , e66.

Nielsen, C.M., and Dymecki, S.M. (2010). Sonic hedgehog is required for vascular outgrowth in the hindbrain choroid plexus. Developmental biology 340, 430-437. 
Nitta, T., Hata, M., Gotoh, S., Seo, Y., Sasaki, H., Hashimoto, N., Furuse, M., and Tsukita, S. (2003). Size-selective loosening of the blood-brain barrier in claudin-5-deficient mice. The Journal of cell biology 161, 653-660.

Nolan, P.M., Peters, J., Strivens, M., Rogers, D., Hagan, J., Spurr, N., Gray, I.C., Vizor, L., Brooker, D., Whitehill, E., et al. (2000). A systematic, genome-wide, phenotype-driven mutagenesis programme for gene function studies in the mouse. Nature genetics 25, 440-443.

Nusslein-Volhard, C. (2012). The zebrafish issue of Development. Development 139, 4099-4103.

Nusslein-Volhard, C., and Wieschaus, E. (1980). Mutations affecting segment number and polarity in Drosophila. Nature 287, 795-801.

Obermüller-Wilén, H. (1973). Fine Structure and Histochemistry of the Choroid Plexus of the Teleost Leuciscus rutilus. Acta Zoologica 54, 219-228.

Obholzer, N., Swinburne, I.A., Schwab, E., Nechiporuk, A.V., Nicolson, T., and Megason, S.G. (2012). Rapid positional cloning of zebrafish mutations by linkage and homozygosity mapping using whole-genome sequencing. Development 139, 4280-4290.

Omura, Y., Korf, H.W., and Oksche, A. (1985). Vascular permeability (problem of the blood-brain barrier) in the pineal organ of the rainbow trout, Salmo gairdneri. Cell and tissue research 239, 599-610.

Oreskovic, D., and Klarica, M. (2010). The formation of cerebrospinal fluid: nearly a hundred years of interpretations and misinterpretations. Brain research reviews 64 , 241-262.

Ormandzhieva, V.K. (2010). Light and dark epithelial cells of the rat choroid plexus. Acta Morphologica et Anthropologica 16, 6.

Page, D.R., and Grossniklaus, U. (2002). The art and design of genetic screens: Arabidopsis thaliana. Nature reviews Genetics 3, 124-136.

Patton, E.E., and Zon, L.I. (2001). The art and design of genetic screens: zebrafish. Nature reviews Genetics 2, 956-966.

Pelegri, F., ed. (2002). Mutagenesis (New York, New York: Oxford University Press).

Rabbani, B., Tekin, M., and Mahdieh, N. (2014). The promise of whole-exome sequencing in medical genetics. Journal of human genetics 59, 5-15.

Redzic, Z.B., Preston, J.E., Duncan, J.A., Chodobski, A., and Szmydynger-Chodobska, J. (2005). The choroid plexus-cerebrospinal fluid system: from development to aging. Current topics in developmental biology $71,1-52$. 
Reichel, V., Miller, D.S., and Fricker, G. (2008). Texas Red transport across rat and dogfish shark (Squalus acanthias) choroid plexus. American journal of physiology Regulatory, integrative and comparative physiology 295, R1311-1319.

Roth, M., Obaidat, A., and Hagenbuch, B. (2012). OATPs, OATs and OCTs: the organic anion and cation transporters of the SLCO and SLC22A gene superfamilies. British journal of pharmacology 165, 1260-1287.

Rozovski, U., Keating, M., and Estrov, Z. (2013). The significance of spliceosome mutations in chronic lymphocytic leukemia. Leukemia \& lymphoma 54, 1364-1366.

Ryan, S., Willer, J., Marjoram, L., Bagwell, J., Mankiewicz, J., Leshchiner, I., Goessling, W., Bagnat, M., and Katsanis, N. (2013). Rapid identification of kidney cyst mutations by whole exome sequencing in zebrafish. Development 140, 4445-4451.

Saengkhae, C., Loetchutinat, C., and Garnier-Suillerot, A. (2003). Kinetic analysis of rhodamines efflux mediated by the multidrug resistance protein (MRP1). Biophysical journal 85, 2006-2014.

Sakka, L., Coll, G., and Chazal, J. (2011). Anatomy and physiology of cerebrospinal fluid. European annals of otorhinolaryngology, head and neck diseases 128, 309-316.

Saunders, N.R., Ek, C.J., Habgood, M.D., and Dziegielewska, K.M. (2008). Barriers in the brain: a renaissance? Trends in neurosciences 31, 279-286.

Saunders, N.R., Knott, G.W., and Dziegielewska, K.M. (2000). Barriers in the immature brain. Cellular and molecular neurobiology 20, 29-40.

Schier, A.F., Neuhauss, S.C., Harvey, M., Malicki, J., Solnica-Krezel, L., Stainier, D.Y., Zwartkruis, F., Abdelilah, S., Stemple, D.L., Rangini, Z., et al. (1996). Mutations affecting the development of the embryonic zebrafish brain. Development 123, 165-178.

Schmidt, R., Strahle, U., and Scholpp, S. (2013). Neurogenesis in zebrafish - from embryo to adult. Neural development 8,3 .

Schwartz, M., and Baruch, K. (2014). The resolution of neuroinflammation in neurodegeneration: leukocyte recruitment via the choroid plexus. The EMBO journal 33, $7-22$.

Sekine, T., Miyazaki, H., and Endou, H. (2006). Molecular physiology of renal organic anion transporters. American journal of physiology Renal physiology 290, F251-261.

Shapiro, A.B., and Ling, V. (1998). Stoichiometry of coupling of rhodamine 123 transport to ATP hydrolysis by P-glycoprotein. European journal of biochemistry / FEBS 254, 189-193. 
Shechter, R., Miller, O., Yovel, G., Rosenzweig, N., London, A., Ruckh, J., Kim, K.W., Klein, E., Kalchenko, V., Bendel, P., et al. (2013). Recruitment of beneficial M2 macrophages to injured spinal cord is orchestrated by remote brain choroid plexus. Immunity $38,555-569$.

Shichijo, S., Nakao, M., Imai, Y., Takasu, H., Kawamoto, M., Niiya, F., Yang, D., Toh, Y., Yamana, H., and Itoh, K. (1998). A gene encoding antigenic peptides of human squamous cell carcinoma recognized by cytotoxic T lymphocytes. The Journal of experimental medicine $187,277-288$.

Siegenthaler, J.A., Ashique, A.M., Zarbalis, K., Patterson, K.P., Hecht, J.H., Kane, M.A., Folias, A.E., Choe, Y., May, S.R., Kume, T., et al. (2009). Retinoic acid from the meninges regulates cortical neuron generation. Cell 139, 597-609.

Sin, O., Michels, H., and Nollen, E.A. (2014). Genetic screens in Caenorhabditis elegans models for neurodegenerative diseases. Biochimica et biophysica acta.

Sivasubbu, S., Balciunas, D., Amsterdam, A., and Ekker, S.C. (2007). Insertional mutagenesis strategies in zebrafish. Genome biology 8 Suppl 1, S9.

Skinner, D.C., and Malpaux, B. (1999). High melatonin concentrations in third ventricular cerebrospinal fluid are not due to Galen vein blood recirculating through the choroid plexus. Endocrinology 140, 4399-4405.

Sparrow, D.B., Clements, M., Withington, S.L., Scott, A.N., Novotny, J., Sillence, D., Kusumi, K., Beddington, R.S., and Dunwoodie, S.L. (2002). Diverse requirements for Notch signalling in mammals. The International journal of developmental biology 46, 365-374.

Spuch, C., and Carro, E. (2011). The p75 neurotrophin receptor localization in blood-CSF barrier: expression in choroid plexus epithelium. BMC neuroscience 12, 39.

St Johnston, D. (2002). The art and design of genetic screens: Drosophila melanogaster. Nature reviews Genetics 3, 176-188.

Stamatovic, S.M., Keep, R.F., and Andjelkovic, A.V. (2008). Brain endothelial cell-cell junctions: how to "open" the blood brain barrier. Current neuropharmacology 6, 179-192.

Stemple, D.L., and Driever, W. (1996). Zebrafish: tools for investigating cellular differentiation. Current opinion in cell biology $8,858-864$.

Strazielle, N., Belin, M.F., and Ghersi-Egea, J.F. (2003). Choroid plexus controls brain availability of anti-HIV nucleoside analogs via pharmacologically inhibitable organic anion transporters. Aids 17, 1473-1485.

Strazielle, N., and Ghersi-Egea, J.F. (2000). Choroid plexus in the central nervous system: biology and physiopathology. Journal of neuropathology and experimental neurology 59, 561-574. 
Streisinger, G., Walker, C., Dower, N., Knauber, D., and Singer, F. (1981). Production of clones of homozygous diploid zebra fish (Brachydanio rerio). Nature 291, 293-296.

Sturrock, R.R. (1979). A morphological study of the development of the mouse choroid plexus. Journal of anatomy 129, 777-793.

Su, L.K., Kinzler, K.W., Vogelstein, B., Preisinger, A.C., Moser, A.R., Luongo, C., Gould, K.A., and Dove, W.F. (1992). Multiple intestinal neoplasia caused by a mutation in the murine homolog of the APC gene. Science 256, 668-670.

Suzuki, T., Inoue, I., Yamagata, T., Morita, N., Furuichi, T., and Yamakawa, K. (2008). Sequential expression of Efhc1/myoclonin1 in choroid plexus and ependymal cell cilia. Biochemical and biophysical research communications 367, 226-233.

Sweet, D.H., Bush, K.T., and Nigam, S.K. (2001). The organic anion transporter family: from physiology to ontogeny and the clinic. American journal of physiology Renal physiology 281, F197-205.

Sweet, D.H., Chan, L.M., Walden, R., Yang, X.P., Miller, D.S., and Pritchard, J.B. (2003). Organic anion transporter 3 (Slc22a8) is a dicarboxylate exchanger indirectly coupled to the $\mathrm{Na}+$ gradient. American journal of physiology Renal physiology 284, F763-769.

Sykes, D., Sweet, D.H., Lowes, S., Nigam, S.K., Pritchard, J.B., and Miller, D.S. (2004). Organic anion transport in choroid plexus from wild-type and organic anion transporter 3 (Slc22a8)-null mice. American journal of physiology Renal physiology 286, F972-978.

Tanackovic, G., Ransijn, A., Thibault, P., Abou Elela, S., Klinck, R., Berson, E.L., Chabot, B., and Rivolta, C. (2011). PRPF mutations are associated with generalized defects in spliceosome formation and pre-mRNA splicing in patients with retinitis pigmentosa. Human molecular genetics 20, 2116-2130.

Tanaka, M., Kokubo, M., and Marunouchi, T. (2007). Asymmetric localization of Notch2 on the microvillous surface in choroid plexus epithelial cells. Histochemistry and cell biology $127,449-456$.

Thambisetty, M., Simmons, A., Velayudhan, L., Hye, A., Campbell, J., Zhang, Y., Wahlund, L.O., Westman, E., Kinsey, A., Guntert, A., et al. (2010). Association of plasma clusterin concentration with severity, pathology, and progression in Alzheimer disease. Archives of general psychiatry 67, 739-748.

Thanos, C.G., Bintz, B., and Emerich, D.F. (2010). Microencapsulated choroid plexus epithelial cell transplants for repair of the brain. Advances in experimental medicine and biology 670, 80-91.

Thisse, C., and Thisse, B. (2008). High-resolution in situ hybridization to whole-mount zebrafish embryos. Nature protocols 3, 59-69. 
Thornberry, N.A. (1997). The caspase family of cysteine proteases. British medical bulletin $53,478-490$.

Trougakos, I.P., Lourda, M., Antonelou, M.H., Kletsas, D., Gorgoulis, V.G., Papassideri, I.S., Zou, Y., Margaritis, L.H., Boothman, D.A., and Gonos, E.S. (2009). Intracellular clusterin inhibits mitochondrial apoptosis by suppressing p53-activating stress signals and stabilizing the cytosolic $\mathrm{Ku} 70$-Bax protein complex. Clinical cancer research : an official journal of the American Association for Cancer Research 15, 48-59.

Tsuiji, H., Iguchi, Y., Furuya, A., Kataoka, A., Hatsuta, H., Atsuta, N., Tanaka, F., Hashizume, Y., Akatsu, H., Murayama, S., et al. (2013). Spliceosome integrity is defective in the motor neuron diseases ALS and SMA. EMBO molecular medicine 5, 221-234.

Tsuneki, K. (1986). A survey of occurrence of about seventeen circumventricular organs in brains of various vertebrates with special reference to lower groups. Journal fur Hirnforschung 27, 441-470.

Umans, R.A., and Taylor, M.R. (2012). Zebrafish as a model to study drug transporters at the blood-brain barrier. Clinical pharmacology and therapeutics 92, 567-570.

Urasaki, A., Morvan, G., and Kawakami, K. (2006). Functional dissection of the Tol2 transposable element identified the minimal cis-sequence and a highly repetitive sequence in the subterminal region essential for transposition. Genetics 174, 639-649.

Vercellino, M., Votta, B., Condello, C., Piacentino, C., Romagnolo, A., Merola, A., Capello, E., Mancardi, G.L., Mutani, R., Giordana, M.T., et al. (2008). Involvement of the choroid plexus in multiple sclerosis autoimmune inflammation: a neuropathological study. Journal of neuroimmunology 199, 133-141.

Villalobos, A.R., Miller, D.S., and Renfro, J.L. (2002). Transepithelial organic anion transport by shark choroid plexus. American journal of physiology Regulatory, integrative and comparative physiology 282, R1308-1316.

Vithana, E.N., Abu-Safieh, L., Allen, M.J., Carey, A., Papaioannou, M., Chakarova, C., Al-Maghtheh, M., Ebenezer, N.D., Willis, C., Moore, A.T., et al. (2001). A human homolog of yeast pre-mRNA splicing gene, PRP31, underlies autosomal dominant retinitis pigmentosa on chromosome 19q13.4 (RP11). Molecular cell 8, 375-381.

Watanabe, M., Kang, Y.J., Davies, L.M., Meghpara, S., Lau, K., Chung, C.Y., Kathiriya, J., Hadjantonakis, A.K., and Monuki, E.S. (2012). BMP4 sufficiency to induce choroid plexus epithelial fate from embryonic stem cell-derived neuroepithelial progenitors. The Journal of neuroscience : the official journal of the Society for Neuroscience 32, 15934-15945.

White, R.M., Sessa, A., Burke, C., Bowman, T., LeBlanc, J., Ceol, C., Bourque, C., Dovey, M., Goessling, W., Burns, C.E., et al. (2008). Transparent adult zebrafish as a tool for in vivo transplantation analysis. Cell stem cell 2, 183-189. 
Wijnholds, J., deLange, E.C., Scheffer, G.L., van den Berg, D.J., Mol, C.A., van der Valk, M., Schinkel, A.H., Scheper, R.J., Breimer, D.D., and Borst, P. (2000). Multidrug resistance protein 1 protects the choroid plexus epithelium and contributes to the blood-cerebrospinal fluid barrier. The Journal of clinical investigation 105, 279-285.

Wilson, S.W., Brand, M., and Eisen, J.S. (2002). Patterning the zebrafish central nervous system. Results and problems in cell differentiation 40, 181-215.

Winata, C.L., Korzh, S., Kondrychyn, I., Zheng, W., Korzh, V., and Gong, Z. (2009). Development of zebrafish swimbladder: The requirement of Hedgehog signaling in specification and organization of the three tissue layers. Developmental biology 331, 222-236.

Wodarczyk, C., Rowe, I., Chiaravalli, M., Pema, M., Qian, F., and Boletta, A. (2009). A novel mouse model reveals that polycystin-1 deficiency in ependyma and choroid plexus results in dysfunctional cilia and hydrocephalus. PloS one 4, e7137.

Wolburg, H., and Paulus, W. (2010). Choroid plexus: biology and pathology. Acta neuropathologica $119,75-88$.

Wolburg, H., Wolburg-Buchholz, K., Liebner, S., and Engelhardt, B. (2001). Claudin-1, claudin-2 and claudin-11 are present in tight junctions of choroid plexus epithelium of the mouse. Neuroscience letters 307, 77-80.

Xie, J., Farage, E., Sugimoto, M., and Anand-Apte, B. (2010). A novel transgenic zebrafish model for blood-brain and blood-retinal barrier development. BMC developmental biology 10, 76 .

Yang, X., Ye, C.Y., Bisaria, A., Tuskan, G.A., and Kalluri, U.C. (2011). Identification of candidate genes in Arabidopsis and Populus cell wall biosynthesis using text-mining, co-expression network analysis and comparative genomics. Plant science : an international journal of experimental plant biology 181, 675-687.

Yin, J., Brocher, J., Fischer, U., and Winkler, C. (2011). Mutant Prpf31 causes pre-mRNA splicing defects and rod photoreceptor cell degeneration in a zebrafish model for Retinitis pigmentosa. Molecular neurodegeneration 6, 56.

Zhang, J., Piontek, J., Wolburg, H., Piehl, C., Liss, M., Otten, C., Christ, A., Willnow, T.E., Blasig, I.E., and Abdelilah-Seyfried, S. (2010). Establishment of a neuroepithelial barrier by Claudin5a is essential for zebrafish brain ventricular lumen expansion. Proceedings of the National Academy of Sciences of the United States of America 107, 1425-1430.

Zhao, Y., Yu, H., and Hu, W. (2014). The regulation of MDM2 oncogene and its impact on human cancers. Acta biochimica et biophysica Sinica 46, 180-189.

Zhou, Y., and Zon, L.I. (2011). The zon laboratory guide to positional cloning in zebrafish. Methods in cell biology 104, 287-309. 
Zlokovic, B.V. (2008). The blood-brain barrier in health and chronic neurodegenerative disorders. Neuron 57, 178-201. 


\section{APPENDIX A. CHAPTER 2 AND CHAPTER 3 SUPPLEMENTAL MOVIES}

All supplemental files are available via links on the ET/D access page.

\section{SupplementalMovie1}

Confocal time lapse imaging of Et(cp:EGFP) $)^{s j 2}$ larvae to observe CP development. Time lapse was initiated at approximately $30 \mathrm{hpf}$ and images were acquired every $30 \mathrm{~min}$ for $65 \mathrm{~h}$. The image is a dorsal view at $20 \times$ magnification.

\section{SupplementalMovie2}

Confocal time lapse imaging of wild-type $E t(c p: E G F P)^{s j 2}$ larvae. Time lapse was initiated at $54 \mathrm{hpf}$ and images were acquired every $30 \mathrm{~min}$ for $48 \mathrm{~h}$. The image is a dorsal view at $20 \times$ magnification.

\section{SupplementalMovie3}

Confocal time lapse imaging of cp140.2 mutant larvae. Time lapse was initiated at $54 \mathrm{hpf}$ and images were acquired every $30 \mathrm{~min}$ for $48 \mathrm{~h}$. The image is a dorsal view at $20 \times$ magnification.

\section{SupplementalMovie4}

Confocal time lapse imaging of $c p 9.6$ mutant larvae. Time lapse was initiated at $54 \mathrm{hpf}$ and images were acquired every $30 \mathrm{~min}$ for $48 \mathrm{~h}$. The image is a dorsal view at $20 \times$ magnification.

\section{SupplementalMovie5}

Confocal time lapse imaging of $c p 79.6$ mutant larvae. Time lapse was initiated at $54 \mathrm{hpf}$ and images were acquired every $30 \mathrm{~min}$ for $48 \mathrm{~h}$. The image is a dorsal view at $20 \times$ magnification.

\section{SupplementalMovie6}

Confocal time lapse imaging of $c p 151.2$ mutant larvae. Time lapse was initiated at $54 \mathrm{hpf}$ and images were acquired every $30 \mathrm{~min}$ for $42 \mathrm{~h}$. The image is a dorsal view at $20 \times$ magnification. 


\section{SupplementalMovie7}

Confocal time lapse imaging of $c p 27.5$ mutant larvae. Time lapse was initiated at $54 \mathrm{hpf}$ and images were acquired every $30 \mathrm{~min}$ for $48 \mathrm{~h}$. The image is a dorsal view at $20 \times$ magnification. 


\section{APPENDIX B. CHAPTER 5 EXTENDED METHODS AND RESULTS OF RNA-SEQ ANALYSIS}

All supplemental files are available via links on the ET/D access page.

RNA-Seq analysis is found in SupplementalFile1. This analysis identifies a number of genes up-regulated and down-regulated due to a point mutation in sart1. The file contains the list of genes with at least one read observed in either one of the samples (wild-type or mutant), genes expressed where the (FPKM $>0.1)$ in both samples, gene ontology for biological processes and molecular function, the Kyoto Encyclopedia of Genes and Genomes (KEGG), and a list of up-regulated spliceosome-related genes.

The primers used for methods described in qRT-PCR on pg. 84 are listed in Table $\mathbf{B - 1}$, and the top ten up-regulated and down-regulated genes with a FPKM $>0.1$ as a result of the sartl mutation are listed in Table B-2. 
Table B-1. Primer Sequences for qRT- PCR.

\begin{tabular}{|c|c|c|c|c|c|}
\hline Gene & Primer & Primer Sequence (5`-3’) & Tm & $\begin{array}{l}\text { GC } \\
\%\end{array}$ & $\begin{array}{l}\text { Product } \\
\text { Length }\end{array}$ \\
\hline \multirow[t]{2}{*}{ sart1 } & Forward & GTCGCAAACTCGCCAAAGAG & 60.1 & 55 & 127 \\
\hline & Reverse & TATGCTTGCCGCTTCTCCTG & 60.5 & 55 & \\
\hline \multirow[t]{2}{*}{$p 53$} & Forward & СТСТСССАССААСАТССАСТ & 59.0 & 55 & 178 \\
\hline & Reverse & ACGTCCACCACCATTTGAAC & 58.7 & 50 & \\
\hline \multirow[t]{2}{*}{$m d m 2$} & Forward & AACCGAGGCAGACTACTGGA & 60.3 & 55 & 105 \\
\hline & Reverse & TCTGGAAGCCAATCAGCTCG & 60.1 & 55 & \\
\hline \multirow[t]{2}{*}{ clu } & Forward & ACACCTCAAGTCTGCTCGAC & 59.7 & 55 & 115 \\
\hline & Reverse & CCTTTGGACATCACTGCCTG & 58.8 & 55 & \\
\hline \multirow[t]{2}{*}{ cldn $5 a$} & Forward & ATGCTGTCTGGCTGACCAAA & 59.9 & 50 & 190 \\
\hline & Reverse & CTTTCTGTTTTCGACGCGCT & 59.8 & 50 & \\
\hline \multirow{2}{*}{ mmp9 } & Forward & AGACTTGGAGTCCTGGCGTT & 61.1 & 55 & 147 \\
\hline & Reverse & ACGCTTCAGATACTCATCCGCTA & 61.3 & 48 & \\
\hline \multirow[t]{2}{*}{$\operatorname{lsm} 7$} & Forward & ACATGCGAGATCCTGATGACC & 59.9 & 52 & 84 \\
\hline & Reverse & CAACAGACGTCCCTCGACAA & 60.0 & 55 & \\
\hline \multirow[t]{2}{*}{$\operatorname{prpf31}$} & Forward & GTCAAGCAGGTCAAGCCTCT & 60.0 & 55 & 221 \\
\hline & Reverse & GCTTGTCTGACTCTGCCACT & 60.0 & 55 & \\
\hline \multirow[t]{2}{*}{ pde6h } & Forward & GACCACTCGCACCTTCAAGA & 60.0 & 55 & 99 \\
\hline & Reverse & ACAGTGATGTCTGTGCCGAG & 60.0 & 55 & \\
\hline \multirow[t]{2}{*}{ opn1sw1 } & Forward & CGATTGCAGGTCTTGTGACG & 59.6 & 55 & 195 \\
\hline & Reverse & GACCCTCGGGAATGTATCTGC & 60.3 & 57 & \\
\hline \multirow[t]{2}{*}{$c r x$} & Forward & CCATTATGCTGTGAACGGGT & 58.3 & 50 & 84 \\
\hline & Reverse & CTCGGAGTGGCTGGGTA & 57.5 & 65 & \\
\hline \multirow[t]{2}{*}{$a c t b 1$} & Forward & TGAATCCCAAAGCCAACAGAG & 58.5 & 48 & 150 \\
\hline & Reverse & TCACACCATCACCAGAGTCC & 59.0 & 55 & \\
\hline
\end{tabular}


Table B-2. Top Ten Up-Regulated and Down-Regulated Genes from RNA-Seq with FPKM $>0.1$ in Both Samples.

\begin{tabular}{|c|c|c|c|}
\hline Name & Gene & Chromosome & $\log _{2}(M U T-W T)$ \\
\hline fibronectin $1 b$ & $f n 1 b$ & 1 & 4.6840 \\
\hline TIMP metallopeptidase inhibitor $2 b$ & timp $2 b$ & 3 & 4.6815 \\
\hline jun $B$ proto-oncogene $b$ & junbb & 3 & 4.5556 \\
\hline $\begin{array}{l}\text { nuclear factor of kappa light } \\
\text { polypeptide gene enhancer in } \\
B \text {-cells inhibitor, alpha a }\end{array}$ & nfkbiaa & 20 & 4.5268 \\
\hline $\begin{array}{l}\text { insulin-like growth factor binding } \\
\text { protein } 1 a\end{array}$ & igfbpla & 20 & 4.4076 \\
\hline $\begin{array}{l}C C A A T / \text { enhancer binding protein } \\
(C / E B P), \text { delta }\end{array}$ & cebpd & 24 & 4.2910 \\
\hline tumor protein p53 & $t p 53$ & 5 & 4.2233 \\
\hline hepcidin antimicrobial peptide & hamp & 16 & 4.1544 \\
\hline $\begin{array}{l}\text { MDM2 oncogene, E3 ubiquitin } \\
\text { protein ligase }\end{array}$ & $m d m 2$ & 4 & 4.0737 \\
\hline clusterin & $c l u$ & 20 & 3.7648 \\
\hline $\begin{array}{l}\text { fibroblast growth factor binding } \\
\text { protein } 2 b\end{array}$ & $f g f b p 2 b$ & 1 & -2.3904 \\
\hline parvalbumin 8 & pvalb8 & 3 & -2.5884 \\
\hline transcobalamin like & tcnl & 5 & -2.7405 \\
\hline $\begin{array}{l}\text { guanine nucleotide binding protein } \\
\text { (G protein), gamma transducing } \\
\text { activity polypeptide } 1\end{array}$ & gngt1 & 19 & -2.7876 \\
\hline actinodin4 & and4 & 14 & -3.0036 \\
\hline \multirow{4}{*}{$\begin{array}{l}\text { creatine kinase, mitochondrial } 2 a \\
\text { (sarcomeric) }\end{array}$} & $c k m t 2 a$ & 10 & -3.1316 \\
\hline & $z g c: 73075$ & 19 & -3.3992 \\
\hline & $z g c: 13622$ & 7 & -3.5883 \\
\hline & $z g c: 73359$ & 3 & -3.8776 \\
\hline
\end{tabular}


Table B-2. (Continued).

\begin{tabular}{lccc}
\hline Name & Gene & Chromosome & $\log _{2}($ MUT-WT) \\
\hline matrilin 1 & matn1 & 19 & -5.4303 \\
\hline
\end{tabular}




\section{VITA}

Hannah Elizabeth Henson was born in, 1987 in Marion, Illinois to Robert and Christie Henson. After graduating from Marion High School in 2005, she went on to attend Union University in Jackson, TN. After completion of her Bachelors of Science degree in Cell Biology in 2009, she continued her education by joining the Integrated Program in Biomedical Sciences at the University of Tennessee Health Science Center in Memphis, TN. Here, she joined the Cancer and Developmental Biology track and became a member of Dr. Michael R. Taylor's lab at St. Jude Children's Research Hospital. Upon approval of her dissertation, she will receive her Doctorate of Philosophy. She will continue her career as a postdoctoral scholar in the lab of Dr. Ann Morris at the University of Kentucky in Lexington, Kentucky. 